Universidade de São Paulo

Instituto de Astronomia, Geofísica e Ciências Atmosféricas

Departamento de Astronomia

\title{
Formação estelar induzida por choques de Supernovas e por Turbulência Magneto-hidrodinâmica
}

\author{
Márcia Regina Moreira Leão \\ Orientadora: Profa. Dra. Elisabete M. de Gouveia Dal Pino
}

São Paulo

2012 
Márcia Regina Moreira Leão

\section{Formação estelar induzida por choques de Supernovas e por Turbulência Magneto-hidrodinâmica}

Tese apresentada ao Departamento de Astronomia do Instituto de Astronomia, Geofísica e Ciências Atmosféricas da Universidade de São Paulo como requisito parcial à obtenção do título de Doutor em Ciências.

Sub-área de concentração: Astrofísica.

Orientadora: Profa. Dra. Elisabete M. de Gouveia Dal Pino

São Paulo 
Ao meu marido Rafael Leão. 


\section{Agradecimentos}

Agradeço primeiramente, ao meu marido Rafael Leão que sempre esteve ao meu lado me apoiando a ajudando. Incentivando sempre a ir em frente.

Agradeço aos meus amigos Reinaldo Santos-Lima e Grzegorz (Greg) Kowal que me ajudaram me ouvindo e discutindo meu trabalho, ao meu amigo Paulo F. Penteado pelas muito úteis aulas de IDL, e agradeço a todos também pelos momentos de descontração. Agradeço principalmente à minha amiga Monique (Baianinha) Alves Cruz e ao meu amigo Rodrigo (Hobbit) Vieira pelo apoio e amizade quando eu mais precisava.

Agradeço à minha orientadora Bete pela paciência e compreensão e ao prof. Alex Lazarian pela colaboração.

Por último, mas não menos importante, agradeço o apoio financeiro do CNPq. 
"Ora (direis) ouvir estrelas! Certo

Perdeste o senso!"E eu vos direi, no entanto,

Que, para ouvi-las, muita vez desperto

E abro as janelas, pálido de espanto...

E conversamos toda a noite, enquanto

A Via Láctea, como um pálio aberto,

Cintila. E, ao vir do sol, saudoso e em pranto,

Inda as procuro pelo céu deserto.

Direis agora: "Tresloucado amigo!

Que conversas com elas? Que sentido

Tem o que dizem, quando estão contigo?"

E eu vos direi: "Amai para entendê-las!

Pois só quem ama pode ter ouvido

Capaz de ouvir e entender estrelas"

(Trecho XIII de Via Láctea - Olavo Bilac)

"Science is a perception of the world around us. Science is a place where what you find in nature pleases you."

(Subrahmanyan Chandrasekhar) 


\section{Sumário}

Resumo iv

Abstract vii

1 Introdução 1

1.1 Roteiro da Tese . . . . . . . . . . . . . . . . . . . 6

2 Formação Estelar induzida por Choques de Supernovas - Revisão Teórica 8

2.1 Nuvens do Meio Interestelar e suas Propriedades . . . . . . . . . . . . . . . 8

2.1.1 Turbulência nas nuvens interestelares . . . . . . . . . . . . . . . . . 12

2.1.2 Equipartição entre Campos Magnéticos e Turbulência na Matéria Neutra Fria $(\mathrm{CNM}) \ldots \ldots \ldots \ldots$

2.1.3 Razão Massa-Fluxo de uma nuvem . . . . . . . . . . . . . . . . . . 17

2.2 Interações entre remanescentes de supernovas e nuvens: uma descrição

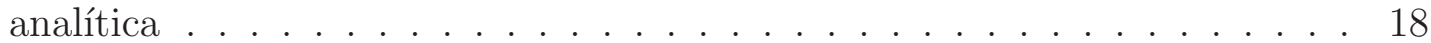

2.2.1 A inclusão do campo magnético na nuvem . . . . . . . . . . . . . 24

2.3 Condições para indução do colapso gravitacional na nuvem . . . . . . . . . 26

2.3.1 O Vínculo da Massa de Jeans . . . . . . . . . . . . . . . . . 27

2.3.2 Vínculo para a não-destruição da nuvem devido ao impacto de um

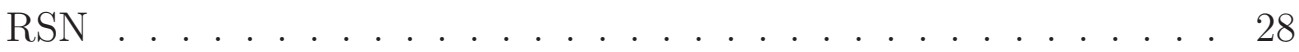

2.3.3 Vínculo da extensão da penetração da frente de choque do RSN na

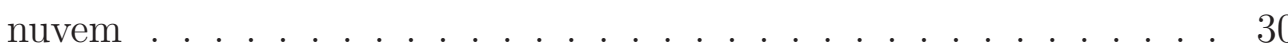


3 Formação Estelar induzida por Choques de SNs - Aplicações a Nuvens Difusas Frias

3.1 Diagramas para a Formação Estelar . . . . . . . . . . . . . . . . 35

3.2 Interação RSN-nuvem: simulações numéricas MHD de nuvens magnetizadas e auto-gravitantes . . . . . . . . . . . . . . . . . . . 4 41

3.3 Aplicação a regiões isoladas do MIS . . . . . . . . . . . . . . . . 46

3.4 Estimando a eficiência de formação estelar devido a interações RSN-nuvens 48

3.5 Conclusões . . . . . . . . . . . . . . . . . . . . . . . . 52

4 Turbulência MHD e a difusão de campos magnéticos por reconexão magnética rápida em nuvens moleculares - Fundamentação teórica $\quad 68$

4.1 Turbulência MHD . . . . . . . . . . . . . . . . . . . . 73

4.2 Reconexão magnética rápida em fluidos turbulentos . . . . . . . . . . . . . 78

4.3 Coeficiente de difusão por reconexão magnética turbulenta . . . . . . . . . 81

5 Formação estelar em nuvens turbulentas e o transporte de Fluxo Magnético 84

5.1 Domínio Computacional e Metodologia Numérica . . . . . . . . . . . . . 85

5.2 Condições Iniciais . . . . . . . . . . . . . . . . . . . . . . . . . . . 89

5.3 Resultados das simulações numéricas . . . . . . . . . . . . . . . . . . . . 92

5.3.1 Modelos com auto-gravidade . . . . . . . . . . . . . . . . 92

5.3.2 Os efeitos da resolução nos resultados . . . . . . . . . . . . . . . . . 101

5.3.3 Modelos sem auto-gravidade . . . . . . . . . . . . . . 102

5.4 Discussões . . . . . . . . . . . . . . . . . . . . . . . . 103

5.4.1 Comparação com os resultados de Santos-Lima et al. (2010) .. . 105

5.4.2 Comparação entre os efeitos da difusividade por reconexão magnética e da resistividade . . . . . . . . . . . . . . . . 107

5.4.3 Potencial gravitacional cilíndrico versus esférico . . . . . . . . . 108

5.4.4 Os efeitos da auto-gravidade sobre o transporte de fluxo magnético devido à difusão por reconexão turbulenta . . . . . . . . . . . . . 110

5.4.5 Os efeitos das condições iniciais da nuvem . . . . . . . . . . . . 111 
5.4.6 Comparação de nossos resultados com as observações . . . . . . . . 113

5.5 Observações finais . . . . . . . . . . . . . . . . . . . 115

6 Conclusões e Perspectivas

6.1 Conclusões - Formação Estelar induzida por Choques de SN . . . . . . . 119

6.2 Conclusões - Formação estelar em nuvens turbulentas e o transporte de Fluxo Magnético . . . . . . . . . . . . . . . . . . . . . . 121

6.3 Perspectivas . . . . . . . . . . . . . . . . . . . . 123

$\begin{array}{ll}\text { A Supernovas e seus remanescentes } & 125\end{array}$

A.1 Tipos de Supernovas . . . . . . . . . . . . . . . . . . 125

A.2 Taxas de explosões de SNs . . . . . . . . . . . . . . . . . . . . . . . 128

A.3 Formação do Remanescente de SN . . . . . . . . . . . . . . . . . . . 130

B Código Numérico 3D MHD-Godunov $\quad 136$

C Local star formation triggered by supernova shocks in magnetized diffuse neutral clouds $\quad 142$

D Magnetic flux transport by turbulent reconnection in astrophysical flows 143

E Cloud core collapse and the role of turbulent magnetic reconnection diffusion

Referências 


\section{Resumo}

Neste trabalho investigamos os efeitos de choques (induzidos por supernovas) e de turbulência magneto-hidrodinâmica no processo de formação estelar. Primeiramente, considerando o impacto de um remanescente de supernova (RSN) com uma nuvem neutra magnetizada derivamos analiticamente um conjunto de condições através das quais estas interações podem levar à formação de estruturas densas capazes de tornarem-se gravitacionalmente instáveis e formar estrelas. Usando estas condições, construímos diagramas do raio do RSN, $R_{R S N}$, versus a densidade inicial da nuvem, $n_{c}$, os quais delimitam um domínio no espaço paramétrico onde a formação estelar é permitida. Estes diagramas foram testados através de simulações numéricas magneto-hidrodinâmicas tridimensionais (3D MHD) onde seguimos a evolução espaço-temporal da interação de um RSN com uma nuvem auto-gravitante. Verificamos que a análise numérica está de acordo com os resultados previstos pelos diagramas. Observamos ainda que a presença de um campo magnético fraco, $\sim 1 \mu \mathrm{G}$, inicialmente homogêneo e perpendicular à velocidade de impacto do RSN, resulta em uma pequena diminuição da região permitida para formação estelar nos diagramas quando comparado a diagramas para nuvens não magnetizadas. Já um campo magnético mais intenso $(\sim 10 \mu \mathrm{G})$ causa um encolhimento significativo nestas, como esperado.

Embora derivados de considerações analíticas simples estes diagramas fornecem uma ferramenta útil para identificar locais onde a formação estelar pode ter sido induzida pelo impacto de uma onda de choque de SN. Aplicações a algumas regiões de nossa Galáxia (como a Grande Concha de CO na direção de Escorpião e a Nuvem Periférica 2 na direção da constelação de Cassiopeia) mostram que a formação estelar nestes locais pode ter sido induzida por uma onda de choque de um RSN em passado recente, quando se consideram valores específicos para as condições iniciais das nuvens impactadas. 
Avaliamos também a eficiência de formação estelar efetiva para estas interações e encontramos que esta é geralmente menor do que os valores observados para a nossa Galáxia (sfe $\sim 0.01-0.3$ ). Este resultado é consistente com outros trabalhos da literatura e também sugere que este mecanismo, embora poderoso para induzir a formação de estruturas, turbulência supersônica e eventualmente formação estelar local, não parece ser suficiente para induzir a formação estelar global em galáxia normais, nem mesmo quando o campo magnético é desprezado.

Além do estudo acima, exploramos ainda a formação estelar considerando a injeção prévia de turbulência (por um mecanismo físico arbitrário) em nuvens magnetizadas. Para uma nuvem ou glóbulo de nuvem molecular formar estrelas deve haver transporte de fluxo magnético das regiões internas mais densas para as regiões externas menos densas da nuvem, de outra forma o colapso poderá ser impedido pela força magnética. Consideramos aqui um novo mecanismo. Reconexão magnética rápida, a qual ocorre em presença de turbulência, pode induzir um processo de difusão eficiente dos campos magnéticos. Neste trabalho investigamos esse processo por meio de simulações numéricas 3D MHD e suas implicações para a formação estelar, estendendo um estudo prévio realizado para nuvens de simetria cilíndrica e sem auto-gravidade (Santos-Lima et al. 2010). Aqui consideramos nuvens mais realistas com potenciais gravitacionais esféricos (devido a estrelas embebidas) e também levando em conta os efeitos da auto-gravidade do gás. Determinamos, pela primeira vez, quais as condições em que o transporte do campo magnético devido à difusão por reconexão turbulenta leva uma nuvem inicialmente subcrítica a tornar-se super-crítica e capaz de colapsar para formar estrelas.

Nossos resultados indicam que a formação de um núcleo supercrítico é resultado de uma complexa interação entre gravidade, auto-gravidade, intensidade do campo magnético e turbulência aproximadamente trans-sônica e trans-Alfvénica. Em particular, a autogravidade favorece a difusão do campo magnético por reconexão turbulenta e, como resultado, seu desacoplamento do gás colapsante torna-se mais eficiente do que quando apenas um campo gravitacional externo está presente. Demonstramos que a difusão por reconexão turbulenta é capaz de remover fluxo magnético da maior parte das nuvens investigadas, porém somente uma minoria desenvolve núcleos aproximadamente críticos ou super- 
críticos, o que é consistente com as observações. A formação destes é restrita ao seguinte intervalo de condições iniciais para as nuvens: razão pressão térmica-pressão magnética, $\beta \sim 1$ a 3 , razões entre a energia turbulenta e a energia magnética $E_{\text {turb }} / E_{\text {mag }} \sim 1.62$ a 2.96, e densidades $50<n<140 \mathrm{~cm}^{-3}$, quando consideramos massas estelares $\mathrm{M}_{\star} \sim 25 \mathrm{M}_{\odot}$, implicando uma massa total da nuvem (gás + estrelas) $\mathrm{M}_{t o t} \lesssim 120 \mathrm{M}_{\odot}$. 


\section{Abstract}

In this work, we have investigated the effects of shocks (induced by supernovae) and magnetohydrodynamical turbulence in the process of star formation. Considering first, the impact of a supernova remnant (RSN) with a neutral magnetized cloud we derived analytically a set of conditions through which these interactions can lead to the formation of dense structures able to become gravitationally unstable and form stars. Using these conditions, we have built diagrams of the RSN radius, $R_{R S N}$, versus the initial cloud density, $n_{c}$, that constrain a domain in the parameter space where star formation is allowed. These diagrams have been also tested by means of three-dimensional magneto-hydrodynamical (3D MHD) numerical simulations where the space-time evolution of a RSN interacting with a self-gravitating cloud is followed. We find that the numerical analysis is in agreement with the results predicted by the diagrams. We have also found that the effects of a weak homogeneous magnetic field $(\sim 1 \mu \mathrm{G})$ approximately perpendicular to the impact velocity of the RSN results only a small decrease of the allowed zone for star formation in the diagrams when compared with the diagrams with non-magnetized clouds. A larger magnetic field $(\sim 10 \mu \mathrm{G})$ on the other hand, causes a significant shrinking of the star formation zone, as one should expect.

Although derived from simple analytical considerations, these diagrams provide a useful tool for identifying sites where star formation could be triggered by the impact of a SN blast wave. Applications of them to a few regions of our own Galaxy (e.g., the large CO shell in the direction of Scorpious, and the Edge Cloud 2 in the direction of the Cassiopeia constellation) have revealed that star formation in those sites could have been triggered by shock waves from SNRs in a recent past, when considering specific values of the RSN radius and the initial conditions in the neutral clouds.

We have also evaluated the effective star formation efficiency for this sort of interac- 
tion and found that it is generally smaller than the observed values in our Galaxy (sfe $~$ $0.01-0.3)$. This result is consistent with previous work in the literature and also suggests that the mechanism presently investigated, though very powerful to drive structure formation, supersonic turbulence and eventually, local star formation, does not seem to be sufficient to drive global star formation in normal star forming galaxies, not even when the magnetic field is neglected.

Besides the study above, we have also explored star formation considering a priori injection of turbulence (by an arbitrary physical mechanism) in magnetized clouds. For a molecular cloud clump to form stars some transport of magnetic flux may be required from the denser, inner regions to the outer regions of the cloud, otherwise this can prevent the gravitational collapse. We have considered here a new mechanism. Fast magnetic reconnection which takes place in the presence of turbulence can induce a process of reconnection diffusion of the magnetic field. In this work, we have investigated this process by means of 3D MHD numerical simulations considering its implications on star formation. We have extended a previous study which considered clouds with cylindrical geometry and no self-gravity (Santos-Lima et al. 2010). Here, we considered more realistic clouds with spherical gravitational potentials (from embedded stars) and also accounted for the effects of the gas self-gravity. We demonstrated that reconnection diffusion takes place. We have also, for the first time, determined the conditions under which reconnection diffusion is efficient enough to make an initially subcritical cloud clump to become supercritical and collapse.

Our results indicate that the formation of a supercritical core is regulated by a complex interplay between gravity, self-gravity, magnetic field strength and nearly transonic and trans-Alfvénic turbulence. In particular, self-gravity helps reconnection diffusion and, as a result, the magnetic field decoupling from the collapsing gas becomes more efficient than in the case when only an external gravitational field is present. We have demonstrated that reconnection diffusion is able to remove magnetic flux from most of the collapsing clumps analysed, but only a few of them develop nearly critical or supercritical cores, which is consistent with the observations. Their formation is restricted to a range of initial conditions for the clouds as follows: thermal to magnetic pressure ratios $\beta \sim 1$ to 3 , 
turbulent to magnetic energy ratios $E_{\text {turb }} / E_{\text {mag }} \sim 1.62$ to 2.96 , and densities $50<n<140$ $\mathrm{cm}^{-3}$, when considering stellar masses $\mathrm{M}_{\star} \sim 25 \mathrm{M}_{\odot}$, implying total (gas+stellar) masses $\mathrm{M}_{t o t} \lesssim 120 \mathrm{M}_{\odot}$ 


\section{Capítulo 1}

\section{Introdução}

"Somewhere, something incredible is waiting to be known."

(Carl Sagan)

Sabemos que o processo de formação estelar se dá em regiões densas, como os glóbulos, dentro de nuvens moleculares gigantes (NMG's, Blitz 1993; Williams, Blitz \& McKee 2000). Isto é devido às suas baixas temperaturas e altas densidades que auxiliam a força gravitacional a tornar-se dominante sobre todas as forças que agem para prevenir o colapso. Contudo, apesar da intensa investigação observacional e teórica, ainda não esta completamente claro como esses glóbulos formam estrelas.

Dois importantes ingredientes estão presentes nas nuvens: campos magnéticos e turbulência. Por um lado, o gradiente de pressão magnética é um importante agente trabalhando contra o colapso gravitacional do gás. A estabilidade de uma nuvem suportada pela pressão magnética pode ser quantificada pela razão massa-fluxo magnético, $M / \Phi \simeq N / B$, onde M é a massa da nuvem, $\Phi$ é o fluxo magnético, B é o campo magnético, e N é a densidade colunar. Esta razão em princípio define até que ponto um campo magnético estático pode impedir uma nuvem de colapsar por ação de sua gravidade (e.g., Nakano \& Nakamura 1978, Crutcher 1999, 2005a, Crutcher \& Troland 2007, Heiles \& Crutcher 2005, Falgarone et al. 2008). Considerando uma nuvem inicialmente esférica, o valor crítico para esta razão é $B_{c r} \simeq 2.57 \times\left(N / 10^{21} \mathrm{~cm}^{-2}\right) \quad \mu \mathrm{G}$, para o gás hidrogênio. Medições do efeito Zeeman (Cap. 2) sugerem que as razões massa-fluxo magnético em núcleos de 
nuvens moleculares são 2.0 vezes maiores do que o valor crítico para o qual a gravidade e o suporte magnético se equilibram (e.g., Crutcher, 2008).

Por outro lado, a presença de turbulência governa a estruturação e fragmentação de nuvens moleculares em todas as escalas, e provavelmente também possui um papel importante durante as diferentes fases da formação estelar (Mac Low \& Klessen, 2004; McKee \& Ostriker, 2007). A observação de larguras de linha que vão de umas poucas a mais de 10 vezes a velocidade do som no meio indicam que os movimentos turbulentos são principalmente supersônicos e trans-sônicos em nuvens moleculares. A turbulência encontra-se aproximadamente em equipartição com o campo magnético no meio interestelar e por esta razão acredita-se que seja trans-Alfvénica (Elmegreen \& Scalo 2004; Heiles \& Troland 2005). De fato, a razão entre a energia turbulenta e a energia magnética para uma nuvem fria neutra é aproximadamente $1.3<E_{\text {turb }} / E_{\text {mag }}<1.9$ (Heiles \& Troland 2005). A formação de estruturas parece estar relacionada à compressão causada pela contínua injeção de energia turbulenta nas nuvens. Mesmo se a turbulência globalmente é forte o suficiente para suportar a nuvem contra a gravidade (Klessen et al., 2000; Mac Low \& Klessen, 2004; Vázquez-Semadeni et al., 2005), ela também pode causar o colapso em pequenas escalas, gerando regiões de alta densidade em meios magnetizados (Heitsch et al., 2001; Nakamura \& Li, 2005; Melioli \& de Gouveia Dal Pino, 2006; Leão et al., 2009) e não magnetizados (Klessen et al., 2000; Elmegreen \& Scalo, 2004). Existem vários mecanismos candidatos para a injeção de energia turbulenta dentro da nuvem. Fontes internas incluem o feedback de estrelas massivas e de baixa massa. Sendo que o primeiro, em particular, pode induzir turbulência através de intensa radiação ionizante, ventos e explosões de supernovas (SNs) (McCray \& Snow, 1979; Melioli \& de Gouveia Dal Pino, 2006; Melioli et al., 2006; Leão et al., 2009). Candidatos externos também incluem choques de remanescentes de supernovas, RSNs (Wada \& Norman, 2001; Elmegreen \& Scalo, 2004; Melioli \& de Gouveia Dal Pino, 2006; Melioli et al., 2006; Leão et al., 2009) e a estrutura espiral galática (Roberts, 1969; Bonnell et al., 2006). Todos estes processos parecem ter poder suficiente para explicar a estrutura e cinemática do meio interestelar (MIS) e poderiam gerar as dispersões de velocidades do gás observadas (Kornreich \& Scalo, 2000). Fontes menos energéticas de turbulência incluem jatos e ventos proto-estelares, expansão 
de regiões HII, e instabilidade magneto-rotacional, mas sua importância relativa é ainda incerta (Joung \& Mac Low, 2006; Ballesteros-Paredes, 2006; Mac Low, 2009).

A primeira parte desta tese (Capítulos 2 e 3) será dedicada ao estudo de um desses mecanismos, considerado um dos principais agentes para induzir turbulência e cujo papel na formação estelar é ainda controverso. Explosões de supernova produzem fortes ondas de choque que atingem nuvens moleculares, comprimindo-as e algumas vezes destruindo-as, mas a compressão pelos choques pode também induzir a formação estelar local (Elmegreen \& Lada 1977; veja também Nakamura \& Li 2005 e referências internas). Existem trabalhos analíticos e numéricos extensos explorando o papel das SNs na geração de turbulência supersônica e estruturas multifásicas no MIS (e.g., Cox \& Smith 1974; McKee \& Cowie 1975; McKee \& Ostriker 1977; Kornreich \& Scalo 2000; Mac Low \& Klessen 2004; Vazquez-Semadeni et al. 2000; de Avillez \& Breitschwerdt 2005) e mesmo em escala galática, na geração de "outflows" em grande escala como chafarizes e ventos galáticos (e.g., de Gouveia Dal Pino \& Medina-Tanco 1999; de Avillez 2000; Heckman et al. 2001; de Avillez \& Berry 2001; Melioli et al. 2008, 2009, de Gouveia Dal Pino et al. 2009, 2010). É provável que o efeito coletivo das SNs seja o fator dominante da turbulência supersônica observada na Galáxia (Norman \& Ferrara, 1996; Mac Low \& Klessen 2004), no entanto, é possível que ele iniba a formação estelar global em vez de induzi-la (Joung \& Mac Low 2006). Aqui, ao invés de examinar os efeitos globais de várias explosões SNs na evolução do MIS e NMGs, exploraremos os efeitos locais dessas interações analiticamente e também por meio de simulações numéricas tridimensionais magneto-hidrodinâmicas (3D MHD). Em Melioli et al. (2006), foi dado um primeiro passo nesta direção examinando-se interações envolvendo um remanescente de supernova (RSN) e uma nuvem não magnetizada e aplicando-se os resultados a uma região de formação estelar onde a associação estelar jovem de $\beta$ Pictoris se formou. Em Leão (2007), as bases desse estudo foram descritas em detalhe e estendidas a nuvens magnetizadas. No Cap. 3, apresentaremos simulações 3D MHD destas interações que confirmam os resultados do estudo analítico e estenderemos essa análise a outras regiões de formação estelar com indicação de interações recentes com frentes de choque de SN (por exemplo, Nuvem Periférica 2 (the Edge Cloud 2), Yasui et al. 2006, Ruffle et al. 2007; e a "Grande Concha de CO", Reynoso \& Mangum 
2001).

Na segunda parte deste trabalho (Capítulos 4 e 5), ao invés de nos concentrarmos em um mecanismo físico específico gerador de turbulência e suas implicações na formação estelar, como fizemos nos dois capítulos anteriores, exploraremos os efeitos globais da presença de turbulência MHD em uma nuvem molecular. O verdadeiro papel que a turbulência MHD possui no colapso de nuvens é ainda bastante debatido. Uma questão importante neste debate é a difusão do campo magnético através de uma nuvem colapsante. Estimativas simples mostram que sob condições de MHD ideal, se todo fluxo magnético fosse dragado com o material colapsante, então o campo magnético em uma proto-estrela seria muitas ordens de magnitude maior do que o que de fato se observa em estrelas TTauri. Este é muitas vezes chamado de "problema do fluxo magnético"(veja Santos-Lima et al. 2012). Para resolver este problema devem-se considerar mecanismos difusivos que possam remover parte do fluxo magnético de uma nuvem colapsante para permitir a formação estelar.

Para lidar com este problema da remoção parcial de campo magnético, tanto em um meio interestelar parcialmente ionizado quanto em nuvens moleculares, pesquisadores usualmente invocam o mecanismo de difusão ambipolar (AD). A AD, introduzida no contexto de formação estelar por Mestel \& Spitzer (1956), vem sendo intensivamente discutida desde então (e.g., Spitzer 1968; Nakano \& Tademaru 1972; Mouschovias 1976, 1977, 1979; Nakano \& Nakamura 1978; Shu 1983; Lizano \& Shu 1989; Fiedler \& Mouschovias 1992, 1993; Li et al. 2008; Fatuzzo \& Adams 2002; Zweibel 2002). Em princípio, a $\mathrm{AD}$ deveria permitir que o fluxo magnético (o qual está acoplado à componente ionizada do gás) fosse redistribuído durante o colapso de regiões de baixa ionização como resultado da colisão entre o gás neutro e o ionizado. Avanços recentes na teoria e nas simulações numéricas, contudo, têm questionado a eficiência deste processo de difusão. Vários autores (Shu et al. 2006, Krasnopolsky et al. 2010, 2011) exploraram a fase de acreção em formação estelar de baixa massa e concluíram que deveria existir uma difusão efetiva aproximadamente três ordens de magnitude maior do que a difusividade Ôhmica para permitir um transporte de fluxo magnético eficiente. Eles verificaram que a AD poderia funcionar somente sob circunstâncias especiais, considerando tamanhos de grãos 
de poeira específicos (veja também Li et al. 2011). Em outras palavras não existe ainda consenso se a AD sozinha é suficiente para resolver o problema de transporte de fluxo magnético em fluxos colapsantes.

Nos Capítulos 4 e 5 exploraremos um novo mecanismo para o transporte de fluxo magnético cujos fundamentos foram originalmente propostos por Lazarian (2005), baseado em reconexão magnética turbulenta (veja também Lazarian \& Vishniac 2009; Santos-Lima et al. 2010; de Gouveia Dal Pino et al. 2011; 2012; Santos-Lima, de Gouveia Dal Pino \& Lazarian 2012). Em condições de MHD ideal há ausência de reconexão magnética e o campo magnético é conservado, mas um importante trabalho demonstrou que na presença de turbulência a reconexão magnética torna-se rápida (Vishniac \& Lazarian 1999). Como a reconexão rápida muda a topologia do campo magnético, pois vários lençóis de corrente se desenvolvem simultaneamente nas pequenas escalas em um fluido turbulento, isto assegura a difusão rápida do campo magnético (Lazarian 2011, SantosLima et al. 2010, Leão et al. 2012).

Recentemente, Santos-Lima et al. (2010; veja também Lazarian et al. 2010; de Gouveia Dal Pino et al. 2011; 2012) exploraram numericamente o papel da reconexão turbulenta no transporte de fluxo magnético das regiões centrais mais densas de uma nuvem molecular para as regiões externas. Eles realizaram simulações MHD de alta resolução tridimensionais de nuvem do meio interestelar considerando um campo gravitacional central gerado por estrelas embebidas. Na presença de turbulência, estas simulações revelaram um aumento da razão massa-fluxo magnético devido ao transporte de fluxo magnético para a periferia da nuvem pela reconexão turbulenta. Com a remoção do excesso de fluxo magnético do núcleo para o envelope da nuvem, o núcleo pode colapsar em vários dos testes analisados, levando à formação estelar. Eles compararam estes resultados com sistemas sem turbulência e nestes casos não encontraram mudanças na razão massa-fluxo magnético, como esperado em sistemas MHD ideais. Esta foi uma evidência adicional de que nos casos com turbulência o transporte de campo magnético era devido à difusão por reconexão turbulenta. Este efeito foi observado tanto quando iniciavam com distribuições de gás e campo magnético em equilíbrio com o campo gravitacional, quanto quando seguiam a evolução de configurações dinamicamente instáveis (gravitacionalmente 
colapsantes). O aumento do potencial gravitacional, bem como a diminuição da magnetização do gás mostram um aumento da segregação da massa e do fluxo magnético no estado final das simulações, suportando a noção de que a difusividade turbulenta relaxa o sistema campo magnético + gás para um estado de mínima energia.

O estudo acima contudo, considerou por simplicidade nuvens com campos gravitacionais cilíndricos. Aqui realizaremos simulações numéricas 3D MHD de alta resolução considerando nuvens mais realistas com potenciais gravitacionais esféricos devido a estrelas embebidas na nuvem e também levaremos em conta os efeitos da auto-gravidade. Veremos que nossos resultados essencialmente confirmam os resultados do trabalho anterior, e sobretudo determinam quais as condições em que o transporte de fluxo magnético por reconexão turbulenta é eficiente o bastante para fazer com que nuvens inicialmente subcríticas tornem-se supercríticas e colapsem, formando estrelas.

\subsection{Roteiro da Tese}

Nos próximos capítulos, os resultados desta tese serão apresentados de acordo com o roteiro descrito a seguir.

No Capítulo 2, discutiremos a estruturação do gás no meio interestelar em suas diversas fases, estudaremos a evolução de choques de remanescentes de supernovas (RSNs) e as condições físicas do impacto entre um RSN e uma nuvem interestelar.Além disso, considerando as condições que são relevantes para a indução de formação estelar nestas interações, derivaremos uma massa de Jeans apropriada para o material da nuvem chocada, determinaremos qual a intensidade que deve ter a interação para que não leve a uma completa destruição da nuvem, e determinaremos também a extensão de penetração da frente de choque da nuvem.

Com isso, no Capítulo 3, construiremos diagramas do raio do RSN em função da densidade da nuvem, para diferentes raios e temperaturas iniciais, os quais determinam o espaço paramétrico onde ocorre a formação estelar induzida por interações entre uma frente de choque de SN e uma nuvem. Esses diagramas serão construídos tanto para interações com remanescentes na fase adiabática (de Sedov), como na fase radiativa. 
Além disso, simulações numéricas magneto-hidrodinâmicas tri-dimensionais (3D MHD) de interações RSN-nuvem para diferentes condições iniciais serão utilizadas para testar esses diagramas construídos analiticamente. Aplicações desses resultados a regiões reais de formação estelar em nossa própria galáxia revelarão que estas poderiam ter sido induzidas por ondas de choque de SN. Com estes diagramas também derivaremos a eficiência de formação estelar para esse tipo de processo.

No Capítulo 4 introduziremos a teoria de turbulência em meios magnetizados (Biskamp 2003; Lazarian 2011) e de reconexão magnética turbulenta seguindo o trabalho de Lazarian \& Vishniac (1999) (veja também Lazarian 2011; Santos-Lima et al. 2010; 2012; Lazarian et al. 2012). Descreveremos também a aplicação deste mecanismo no transporte de fluxo magnético no interior de nuvens moleculares turbulentas.

No Capítulo 5, apresentaremos os estudos numéricos que realizamos com o objetivo de testar essa teoria de transporte de fluxo magnético por reconexão turbulenta em nuvens moleculares interestelares e os efeitos esperados da mesma sobre a formação estelar. Descreveremos primeiro a metodologia numérica que empregamos para realizar simulações 3D MHD de núcleos de nuvens moleculares ou glóbulos em presença de turbulência. Em seguida, apresentaremos os resultados da difusão de fluxo magnético pela turbulência considerando nuvens moleculares com auto-gravidade, iniciadas tanto em equilíbrio magnetohidrostático, quanto fora deste, e considerando-se nuvens com ou sem a presença de um potencial gravitacional esférico central devido a estrelas embebidas. Serão também realizadas comparações qualitativas de nossos resultados numéricos com 4 glóbulos densos (B1, B2172, L1448CO e L1544) observados por Crutcher et al. (2009) para os quais as razões massa-fluxo magnético foram obtidas das observações. Veremos que o mecanismo de difusão dos campos magnéticos aqui descrito, por reconexão turbulenta, parece ser bem mais eficiente e não apresenta as dificuldades encontradas pela difusão ambipolar e constitui, portanto, um novo paradigma para a teoria de formação estelar.

Finalmente, no Capítulo 6 as principais conclusões deste trabalho serão sumarizadas e as perspectivas futuras serão apresentadas. 


\section{Capítulo 2}

\section{Formação Estelar induzida por Choques de Supernovas - Revisão Teórica}

"Imagination will often carry us to worlds that never were. But without it we go nowhere."

(Carl Sagan)

Neste Capítulo apresentaremos a base conceitual e teórica para a realização da primeira parte deste trabalho relativa à formação de estrelas induzida pelo choque de RSNs em nuvens interestelares difusas. Para tal, começaremos com uma breve descrição dos principais componentes do meio interestelar.

\subsection{Nuvens do Meio Interestelar e suas Propriedades}

O meio interestelar (MIS) é o nome dado a tudo que preenche o espaço entre as estrelas numa galáxia. Basicamente é um gás extremamente tênue composto principalmente por hidrogênio. Responsável por 90\% da composição da matéria interestelar este aparece em suas diversas formas, molecular, neutro e ionizado. Encontram-se também o hélio (HeI, HeII) e traços de carbono, oxigênio, ferro e outros elementos mais pesados. Outros 
componentes do MIS são moléculas (e.g., CO e CS), poeira e raios cósmicos. O meio é também permeado por campos de radiação e campos magnéticos (Schulz, 2005).

O meio interestelar é um lugar agitado. Aquecido por choques de supernovas (SNs) e ventos estelares, constantemente resfriando e colidindo com nuvens moleculares densas nas quais se formam novas estrelas, que por sua vez geram novos ventos e novas SNs. O material destas nuvens é formado por emaranhado denso e frio de gás atômico e molecular imerso num meio mais quente, observando-se uma grande variedade de densidades e temperaturas. Dividindo o meio em faixas de densidade e temperatura obtemos as diversas componentes ou fases do MIS. Um modelo bastante aceito é a versão de três fases em equilíbrio global de pressão: o gás denso frio (meio dominado por hidrogênio atômico neutro HI e por nuvens difusas), a componente morna (ou meio inter-nuvens morno, neutro e ionizado) e o gás quente de baixa densidade (componente quente, ou coronal). As nuvens escuras densas, também denominadas nuvens moleculares, podem ser incluídas como uma quarta fase transiente ligadas à formação estelar (Cox \& Smith, 1974; McKee \& Otriker, 1977; Cox, 2005). Da fase mais fria para a mais quente, as mesmas possuem as seguintes características (Dopita \& Sutherland, 2003):

- Nuvens Moleculares (MC-Molecular Clouds). A temperatura nestes enormes complexos de poeira e gás encontra-se em média entre $10 \mathrm{~K}$ e $100 \mathrm{~K}$, apresentando densidades maiores que $10^{3} \mathrm{~cm}^{-3}$, massas de $10^{5}$ a $10^{6} \mathrm{M}_{\odot}$ e dimensões que podem atingir várias dezenas de parsecs (veja por exemplo a revisão de Blitz 1993). Nuvens moleculares gigantes contém uma grande variedade de sub-estruturas (glóbulos com densidades $n \sim 10^{3} \mathrm{~cm}^{-3}$ na escala de parsecs e núcleos com densidades superiores a $10^{4} \mathrm{~cm}^{-3}$ na escala de sub-parsecs) (Vázquez-Semadeni 2010). A intensidade dos campos magnéticos nestas nuvens pode variar de $1 \mu G$ para nuvens gigantes de dezenas de parsecs até $100 \mu \mathrm{G}$ para os pequenos glóbulos de décimos de parsecs nelas embebidos. Embora esta fase ocupe somente uma pequena fração do volume total, da ordem de $1 \%$, estas contem uma fração considerável $(\sim 30-60 \%)$ da massa total contida no meio interestelar Galático. Muitas destas nuvens moleculares são gravitacionalmente ligadas e estão diretamente associadas ao processo de formação 
estelar de forma que seus núcleos densos podem estar colapsando para formar novas gerações de estrelas.

- Meio Neutro Frio (CNM - Cold Neutral Medium). O gás deste meio apresenta-se distribuído em densas nuvens muitas das quais com a forma de filamentos ocupando 1-5\% do volume total do disco da Galáxia (e.g., de Avillez \& Breitschwerdt 2005). Este meio, com temperaturas em média um pouco mais elevadas que as das MCs $(T \sim 100-200 \mathrm{~K})$ não encontra-se gravitacionalmente ligado, mas está em um balanço de pressão com os demais componentes do MIS (Wolfire et al. 2003). A densidade das nuvens do CNM é $1-100 \mathrm{~cm}^{-3}$ e suas massas são da ordem de $10^{3} \mathrm{M}_{\odot}$, possuindo dimensões de dezenas de parsecs. O campo magnético associado a estas nuvens fica entre $1-10 \mu \mathrm{G}$. O meio neutro frio está também associado a regiões de formação estelar visto que as nuvens moleculares, os sítios onde formam-se estrelas propriamente, podem ser formadas a partir destas nuvens (Haynes \& Broeils, 1997).

- Meio Neutro Aquecido/Morno (WNM - Warm Neutral Medium). As nuvens do WNM são formadas basicamente por hidrogênio neutro em sua forma atômica, tal como o CNM. Esta fase, traçada pela emissão de $21 \mathrm{~cm}$ do HI (Carrow \& Ostlie 2007), ocupa uma fração substancial do volume total $(\sim 30-60 \%)$ do gás do disco de nossa Galáxia e de outras galáxias (Dopita \& Sutherland, 2003). O gás está localizado principalmente em regiões de fotoionização. Em regiões distantes de estrelas jovens este possui $T \sim 5000-10000 \mathrm{~K}$ (Wolfe, Prochaska \& Gawiser 2003; Carswell et al. 2012), e $n \sim 0.1-1.0 \mathrm{~cm}^{-3}$ (Dopita \& Sutherland, 2003; Cox, 2005) . Na Via Láctea, aproximadamente $75 \%$ de todo hidrogênio encontra-se nesta componente dependendo da localização, abrangendo uma grande porcentagem da massa do MIS (Haynes \& Broeils, 1997)

- Meio Ionizado Aquecido/Morno (WIM - Warm ionized Medium). O meio ionizado morno, também chamado de gás ionizado difuso, é um dos maiores componentes do meio interestelar em nossa Galáxia e em outras (Mathis, 2000; Rand, 1997). Composta por hidrogênio quase completamente ionizado, esta fase é associada a 
regiões HII onde o gás é ionizado por estrelas jovens quentes (Madsenet al., 2002; Mathis, 2000; Reynolds et al., 2000). Na Via Láctea é caracterizado por regiões com baixas densidades $n \sim 0.01-1.0 \mathrm{~cm}^{-3}$ e temperaturas $T \sim 7000-13000 \mathrm{~K}$ dependendo da abundância do gás e da temperatura das estrelas presentes (e.g. Reynolds 1990; Reynolds et al. 2000; Mishra et al. 2013). Ocupa somente 2 $4 \%$ do volume total do disco, porém acima de uma altura de $1.5 \mathrm{kpc}$ ocupa uma fração significantiva (20\%) do volume total, encontra-se portanto, distribuído em um disco espesso, estendendo-se a poucos kpc acima e abaixo do plano Galático com uma queda aproximadamente exponencial da densidade com a altura (Readhead \& Duffett-Smith 1975; Reynolds 1989; Nordgren et al. 1992). Este não é um meio homogêneo e suave mas está dividido em nuvens e camadas discretas (Reynolds 1977, 1991; Pynzar 1993).

- Meio Ionizado Quente (HIM - Hot Ionised Medium). Esta fase é aquecida por choques resultantes de explosões de supernovas ou fortes ventos estelares, tendo $T>$ $10^{6} \mathrm{~K}$ devido à constante injeção de energia no meio. Com densidades muito baixas de $10^{-4}$ a $10^{-2} \mathrm{~cm}^{-3}$ e longo tempo de resfriamento o gás ocupa uma grande fração do MIS. No plano central das galáxias o gás quente tem um fator de preenchimento pequeno, estando confinado dentro de bolhas, a maioria em regiões HII. Nestas regiões, para $T>5 \times 10^{6} \mathrm{~K}$, esta fase torna-se detectável em uma difusa emissão de raio-X mole graças às altas pressões em que se encontra. Acima e abaixo do plano galático, o gás quente leva à formação dos chafarizes galáticos empurrando gás mais denso para fora do plano do disco (e.g. Melioli et al. 2008, 2009). Embora pouco gás encontre-se nesta fase ele ocupa uma fração muito grande do volume disponível, em média $(\sim 50 \%)$ do volume total (McCray, 1985). Em nossa Galáxia eventualmente esta fase funde-se ao halo quente em uma escala de altura de 2 - $5 \mathrm{kpc}$.

Como já foi mencionado, nenhuma destas componentes encontra-se estática no tempo ou espaço, e a matéria está constantemente fluindo entre as diversas fases.

As nuvens moleculares gigantes parecem formar-se por acúmulo de nuvens neutras ao longo das linhas do campo magnético em larga escala (Crutcher, 2005). Na primeira parte 
deste trabalho, nas próximas secções deste Cap. e Cap. 3, discutiremos formação estelar considerando escalas de algumas dezenas de parsecs de matéria difusa neutra (CNM) e examinaremos o impacto de um remanescente de supernova (RSN) com estas nuvens frias e a consequente formação de estruturas densas que poderão vir a colapsar para formar estrelas. Examinaremos também como a presença dos campos magnéticos influenciam esse processo. Já, na segunda parte, Caps. 4 e 5, consideraremos regiões menores dentro destas nuvens de décimos a alguns poucos parsecs, e investigaremos sua evolução sob a ação de turbulência MHD. Nosso objetivo será verificar como se formam os caroços densos e frios em nuvens moleculares. Para isso analisaremos o processo de transporte de campo magnético do centro dessas nuvens para seus envelopes através da reconexão magnética turbulenta rápida.

\subsubsection{Turbulência nas nuvens interestelares}

Aparentemente nuvens moleculares são estruturas relativamente transientes, evoluindo dinamicamente, produzidas no meio difuso de HI por movimentos compressíveis de origem gravitacional ou turbulenta, ou uma combinação de ambos (e.g., Hartmann et al. 2001; Ballestero-Paredes et al. 2007). De fato, observações de larguras de linhas espectrais supersônicas nas MCs indicam a presença de turbulência supersônica nestas nuvens com uma variedade de estruturas em todas as escalas e com números de Mach $M \sim 10$ (Larson 1981; Blitz \& Williams 1999; Elmegreen \& Scalo 2004; Goodman et al. 1998; Williams et al. 2000; Lazarian \& Esquivel 2003).

Simulações numéricas recentes em domínios periódicos têm lançado alguma luz no papel da turbulência na evolução de nuvens moleculares e na formação de estrelas dentro delas (veja também Cap. 4). Elas sugerem que a contínua injeção de movimentos turbulentos, mantidos por mecanismos externos e internos (Cap. 1), pode suportar uma nuvem globalmente contra o colapso gravitacional tal que o efeito resultante seria o de inibir o colapso e isto poderia explicar a baixa eficiência de formação estelar global observada na Galáxia (Klessen et al. 2000; Mac Low \& Klessen 2004; Vazquez-Semandeni et al. 2005). Por outro lado, a turbulência supersônica é também capaz de produzir aumentos de densi- 
dade no gás os quais podem induzir o colapso local do gás e formar estrelas, tanto no meio não magnetizado (Klessen et al. 2000; Elmegreen \& Scalo 2004), quanto no magnetizado (Heitsch et al. 2001; Nakamura \& Li 2005).

Neste Capítulo e no próximo, nosso foco serão as interações de ondas de choques de remanescentes de supernovas com nuvens interestelares. Estes choques, como veremos, induzem compressão e movimentos turbulentos supersônicos nas nuvens. Antes de prosseguirmos, faremos uma breve descrição conceitual sobre turbulência subsônica e supersônica. Uma descrição quantitativa da turbulência é muitas vezes vinculada a fluxos incompressíveis, ou subsônicos (Shu 1992). O parâmetro que caracteriza o estado turbulento ou laminar de um fluido é o número de Reynolds (Re), o qual é dado pela razão entre o termo de transporte e o termo de difusão da equação que descreve a evolução da helicidade cinética $(\nabla \times v)$ de um fluido, isto é, $R_{e}=v L / \nu$, onde $L$ e $v$ são, respectivamente, a escala de comprimento e a velocidade características do fluido, e $\nu$ é sua viscosidade cinemática, a qual dá a medida da quantidade de momento difundida de um elemento de fluido a outro. Portanto, um fluido é turbulento quando $R_{e}>1$ e laminar, quando do contrário.

A descrição da turbulência hidrodinâmica é dada pela teoria de Kolmogorov (1941) (veja também Landau \& Lifshitz (1987), §33 e Frisch 1995). Nesta, a energia é injetada nas grandes escalas do sistema criando grandes vórtices, os quais possuem grandes números de Reynolds e portanto, não dissipam energia através da viscosidade, mas transferem energia para vórtices menores. O processo continua até que a cascata atinja vórtices bem pequenos para os quais a viscosidade é dominante (e onde $R e \sim 1$ ) e portanto, a sua energia é totalmente dissipada em um período de rotação do vórtice.

Uma análise dimensional da injeção de energia nas grandes escalas nos dá a lei de Kolmogorov (Landau \& Lifshitz 1966; Shu 1992; veja também Kolmogorov 1941)

$$
E(k) \propto k^{-5 / 3}
$$

onde $k=2 \pi / l$ é o numero de onda e é proporcional ao inverso do tamanho (ou escala) $l$ de um vórtice. 


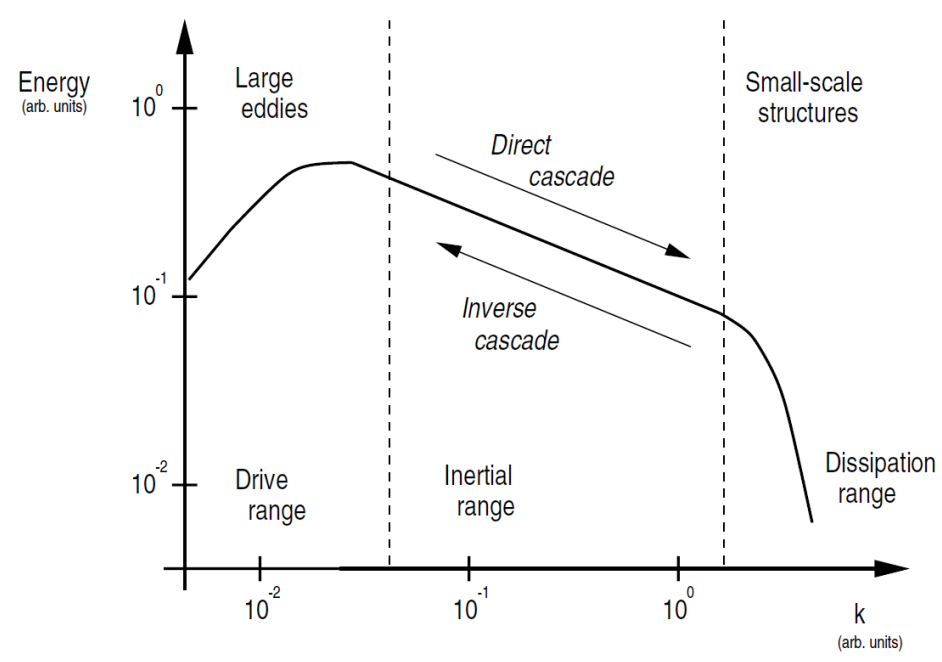

Figura 2.1: Esboço do espectro de potência da turbulência Kolmogorov.

Na Figura 2.1 vemos um esboço do espectro de potência de Kolmogorov mostrando a escala de injeção e a escala de dissipação da turbulência e a região entre essas duas escalas denominada inercial onde efetivamente a energia cascateia das maiores para as menores escalas. A principal suposição por trás desta lei é de que a energia turbulenta $E$ contida nos vórtices não possui outro meio de ser dissipada além de interagir com vórtices cada vez menores transferindo energia a uma taxa constante para escalas cada vez menores, isto é, para números de onda cada vez maiores, até que a dissipação viscosa aja nas menores escalas e transforme a energia cinética em calor. Como a viscosidade age somente nas menores escalas não é possível um vórtice em grande escala dissipar sua energia diretamente, esta vai cascateando entre a escala de injeção e a escala de dissipação de acordo com a lei acima (veja a Fig. 2.1).

Porém, no meio interestelar detectamos frequentemente fluxos compressíveis, através das observações de grandes larguras das linhas espectrais, sugerindo que se tratam de fluidos turbulentos supersônicos, o que muda completamente a situação. Nestes, as pequenas e as grandes escalas podem interagir diretamente por meio de ondas de compressão (ondas sonoras), choques e viscosidade de massa, sem ter que passar pelas escalas intermediárias (Shu 1992). A compressibilidade gera um acoplamento em todas as escalas enquanto que os choques levam a uma dissipação de energia nas grandes escalas de forma que a 
equação anterior não é mais válida. Uma boa descrição pode ser dada pela equação para turbulência de Burgers (Burgers 1974; Menon \& Pego 2007),

$$
E(k) \propto k^{-2},
$$

onde este espectro de potência pode ser derivado da transformada de Fourier para a estrutura dos choques.

Uma outra forma de interpretar as larguras de linhas observadas é a inclusão de campo magnético, pois este torna o sistema mais elástico. Ou seja, o fluido pode ser supersônico (isto é, mover-se com velocidade maior que a do som) e no entanto, possuir velocidade menor do que aquela que caracteriza um fluido magnetizado, isto é, a velocidade de Alfvén, $v_{A}=B /(4 \pi \rho)^{1 / 2}$. Neste caso o fluido será sub-Alfvénico. Como consequência, o transporte de informação (à velocidade de Alfvén) ocorre antes da movimentação do fluido permitindo mudanças de direção suaves (ao invés de violentas, como no caso de fluidos supersônicos e super-Alfvénicos onde há ocorrência de choques). Devemos levar em consideração, contudo, que campos magnéticos ao serem torcidos tendem a restaurar seu estado original, pois as forças magnéticas agem contra torções fortes. Desta forma, quando um fluido move-se mais lentamente que a velocidade de Alfvén, as oscilações assumem uma característica de ondas mais do que de vórtices, ou seja, em um meio magnetizado, a turbulência supersônica e sub-Alfvénica se parece mais com muitas ondas MHD comprimidas do que com vórtices. Discutiremos turbulência MHD em maior detalhe no Cap. 4.

\subsubsection{Equipartição entre Campos Magnéticos e Turbulência na Matéria Neutra Fria (CNM)}

Podemos determinar a intensidade do campo magnético no gás interestelar difuso e em nuvens de vários modos. Existem dois métodos principais de medir o campo: efeito Zeeman, em linhas espectrais, o qual permite medir a componente do campo magnético ao longo da linha de visada, $B_{l o s}$ (do inglês line-of-sight); e o método Chandrasekhar- 
Fermi $^{1}$ (Chandrasekhar \& Fermi 1953), o qual permite medir a componente do campo no plano do céu, $B_{p o s}$ (do inglês plane of the sky), através de medidas de polarização da poeira e da polarização linear de linhas espectrais (Goldreich \& Kylafis 1981; veja também Crutcher 2005 e Heiles \& Crutcher 2005).

Não há correlações óbvias entre o campo magnético observado ao longo da linha de visada $B_{l o s}$ com qualquer quantidade, incluindo a densidade de coluna observada $N_{o b s}$, largura de linha, ou $T_{k}$. No entanto, podemos comparar as diferentes densidades de energia.

Em Heiles \& Troland (2005) cada componente do CNM é caracterizado por valores medidos não só do campo magnético, mas também da temperatura, densidade de coluna, e dispersão de velocidade. Isso nos permite comparar densidades de energia. Uma maneira de fazer isto é com a razão entre as pressões térmica e magnética, o parâmetro $\beta_{t h}=\rho c_{s}^{2} /\left(B^{2} / 8 \pi\right)$, que é o mesmo que compararmos as densidades de energia térmica e magnética. Podemos também definir a razão entre a densidade de energia turbulenta e a densidade de energia magnética. Usando valores médios para o meio difuso frio (Heiles \& Troland 2005):

$$
\begin{gathered}
T=50 K \\
\Delta v_{\text {turb }, 1 d}=1.2 \mathrm{~km} \cdot \mathrm{s}^{-1} \\
B_{\text {tot }}=6.0 \mu \mathrm{G} .
\end{gathered}
$$

Com estes valores obtemos:

$$
\beta_{t h}=\frac{8 \pi \rho c_{s}^{2}}{B^{2}}=0.29
$$

\footnotetext{
${ }^{1} \mathrm{O}$ método Chandrasekhar-Fermi faz uma estimativa do campo magnético do MIS baseado nas dispersões do ângulo de polarização e da velocidade do gás, comparando as flutuações na direção de $B_{\text {pos }}$ com as do campo de velocidades e assumindo que estas perturbações do campo são Alfvénicas e que a velocidade rms é isotrópica.
} 


$$
\beta_{\text {turb }}=\frac{E_{\text {turb }}}{E_{\text {mag }}}=M_{A, t u r b}^{2}=1.3
$$

Onde T é a temperatura média, $\Delta v_{t u r b, 1 d}$ é a velocidade média de dispersão da turbulência em uma dimensão, e $B_{t o t}$ é o campo magnético total médio para este meio. Estes valores sugerem que a pressão térmica é menos dominante que a magnética, mas a turbulenta é comparável a esta última no CNM. Estes valores, contudo, não são representativos visto que nem toda a matéria fria no meio interestelar assume tais valores, assim esperamos uma dispersão considerável para estes parâmetros. Neste trabalho usaremos $\beta_{t h}$ entre 0.3 e 3.0 obtendo $\beta_{\text {turb }}$ entre 1.9 e 2.6 (veja também Cap. 5).

Todas as outras fases difusas do MIS são menos densas do que a CNM. Por exemplo, tanto o meio morno neutro quanto o ionizado são aproximadamente duas ordens de magnitude menos densos de forma que a intensidade do campo magnético não depende muito da densidade. Em contraste, em nuvens moleculares densas, devido à maior importância da auto-gravidade, após um tempo de evolução dinâmica, a densidade volumétrica $\rho$ e a intensidade do campo magnético $B$ podem apresentar algum grau de correlação, $B \approx \rho^{\kappa}$ (conforme previsto pelo congelamento do fluxo de campo magnético com o gás em MHD ideal; veja adiante), com $\kappa$ tendo um valor representativo entre zero e um. Estudos observacionais revelam que a força do campo no meio interestelar permanece invariante em $\sim 6 \mu \mathrm{G}$ entre $0.1 \mathrm{~cm}^{-3}<n(H)<10^{3} \mathrm{~cm}^{-3}$, mas é da forma $B \approx \rho^{0.4-0.5}$ para $10^{3}$ $\mathrm{cm}^{-3}<n\left(H_{2}\right)<10^{8} \mathrm{~cm}^{-3}$ (Crutcher, 2005a,b; Crutcher \& Troland, 2006).

\subsubsection{Razão Massa-Fluxo de uma nuvem}

Considerando o teorema do Virial para uma nuvem de gás magnetizada e assumindo congelamento do fluxo magnético à matéria ionizada (isto é, MHD ideal), podemos derivar a razão entre a energia gravitacional e a energia magnética Esta relação também pode ser expressa através da razão massa-fluxo magnético, $M / \Phi$, a qual é observável, em princípio, para nuvens do MIS (Crutcher 2005; McKee \& Ostriker 2007). Podemos ainda determinar o valor minimo que deve ter o campo magnético em uma nuvem para que 
este suporte a nuvem contra o colapso gravitacional . Esse valor, corresponde a um valor crítico da razão massa-fluxo dado por (Strittmatter 1966; Mouschovias \& Spitzer 1976; Nakano \& Nakamura 1978):

$$
\left(\frac{M}{\phi}\right)_{c r}=\frac{c_{\Phi}}{G^{1 / 2}} \quad g \cdot c m^{2} \cdot G^{-1}
$$

onde $c_{\Phi}$ é um fator próximo de 1 que depende da geometria e da distribuição da densidade e do campo magnético da nuvem. Strittmatter (1966) obteve $c_{\Phi} \simeq 0.2$ para nuvens esféricas (excentricidade $\rightarrow 0$ ) com densidade e campo uniformes e $c_{\Phi} \simeq 0.11$ para nuvens oblatas (excentricidade $\rightarrow 1$ ). Enquanto que Nakano \& Nakamura (1978) encontraram $c_{\Phi}=1 /(2 \pi) \simeq 0.16$ através da análise de perturbações lineares em nuvens com uma estratificação da densidade na direção z. A nuvem é dita subcrítica ou estável se é suportada pelo campo magnético, e supercrítica se a gravidade é dominante. Se não houver outro mecanismo de suporte atuando ao longo das linhas de campo, uma nuvem suportada magneticamente irá evoluir para a forma de um esferoide oblato, como uma panqueca, e se manterá em equilíbrio (formando um glóbulo de Bok). Porém, se sofrer a ação de agentes externos, como uma onda de choque de uma supernova, por exemplo, sairá do equilíbrio e poderá vir a colapsar gravitacionalmente, como veremos adiante.

\subsection{Interações entre remanescentes de supernovas e nuvens: uma descrição analítica}

Iremos considerar agora as equações que são relevantes para o estudo da interação entre um remanescente de supernova e uma nuvem. Uma dedução detalhada das equações que serão utilizadas encontra-se em Leão (2007) (veja também o Ap. A). Uma explosão de SN tipo II gera uma onda de choque esférica que varre o meio interestelar levando à formação de um remanescente de supernova (RSN). A interação entre um RSN e uma nuvem pode comprimir o gás o suficiente para induzir o colapso da nuvem. Para descrever analiticamente esta interação iremos considerar uma nuvem neutra difusa com densidade inicial homogênea e temperatura constante. Após o impacto, um choque interno propaga- 
se na nuvem com uma velocidade $v_{c s}$. A pressão dinâmica da onda de choque, $\sim n_{s h} v_{R S N}^{2}$, deve ser comparável à pressão dinâmica atrás do choque na nuvem, $\sim n_{c} v_{c s}^{2}$ e isto resulta em uma relação bem conhecida para $v_{c s}$ no caso de um choque plano:

$$
v_{c s} \sim v_{R S N}\left(\frac{n_{s h}}{n_{c}}\right)^{1 / 2}
$$

onde $\left(\frac{n_{s h}}{n_{c}}\right)=\chi$ é o contraste de densidade entre a casca ou 'shell' do RSN e a nuvem, $n_{s h}$ é a densidade da 'shell' e $n_{c}$ é a densidade do gás da nuvem. Como discutido em Leão (2007) (veja também a seç̧ão A.3 do Apêndice A) durante sua evolução um RSN passa por dois regimes principais: um adiabático (ou Sedov-Taylor) e posteriormente um regime radiativo. Quando o RSN está ainda na fase adiabática pode-se demonstrar que (Leão, 2007, e A.22 da secção A.3):

$$
v_{c s, a}(R) \sim 43 \frac{E_{51}^{1 / 2}}{R_{R S N, 50}^{3 / 2} n_{c, 10}^{1 / 2}} \mathrm{~km} / \mathrm{s}
$$

onde $n_{c, 10}$ é a densidade da nuvem em unidades de $10 \mathrm{~cm}^{-3}, E_{51}$ é a energia da SN em unidades de $10^{51} \mathrm{erg}, R_{R S N, 50}$ é o raio da 'shell' do RSN em unidades de 50 pc. Quando o RSN entra na fase radiativa sua velocidade de expansão é descrita pela equação A.33 da secção A.3:

$$
v_{c s, r} \sim 47 \frac{E_{51}^{0.8} f_{10}^{1 / 2}}{R_{R S N, 50}^{5 / 2} n_{c, 10}^{1 / 2} n^{0.41}} \quad \mathrm{~km} / \mathrm{s}
$$

onde $f_{10}\left(f=\left(\frac{n_{s h}}{n}\right)\right)$ é o contraste de densidade entre a 'shell' do RSN e a densidade do meio interestelar em unidades de 10 e $n$ é a densidade do meio ambiente.

Porém, as equações acima são válidas somente para um choque plano. Para as interações entre o RSN e a nuvem esféricos precisamos considerar os efeitos da curvatura tanto da frente de choque do RSN, quanto da nuvem. A velocidade instantânea do gás chocado movendo-se em direção ao centro da nuvem, $v_{c s}$, é somente uma fração da velocidade de expansão do RSN e depende do contraste de densidade, $\chi$, entre a 'shell' e a nuvem (como no caso de choque plano) e do ângulo $\gamma$ entre o vetor velocidade do RSN e a linha que liga o centro da nuvem e o ponto de contato instantâneo entre a nuvem e o RSN 


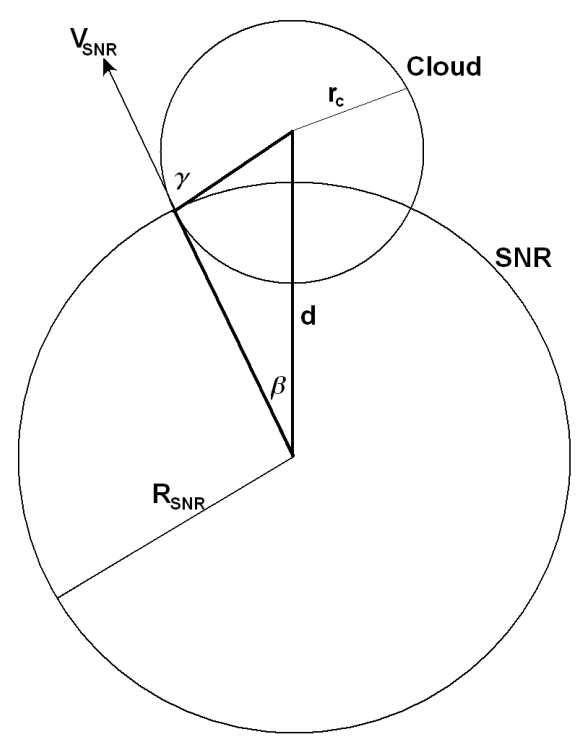

Figura 2.2: Diagrama esquemático da interação entre um RSN e uma nuvem. O RSN expande e impacta a nuvem. Os ângulos $\beta$ e $\gamma$ são funções do tempo, da velocidade do RSN, e dos raios da nuvem e do RSN, como indicado pelas equações no texto.

(Fig. 2.2). Vemos que em $t=0$, isto é, quando o RSN toca a nuvem, estas duas linhas são coincidentes e $\gamma=0$, então $v_{c s}=\chi^{1 / 2} v_{R S N}$. Posteriormente, em um tempo $t_{c, R S N}$ quando o RSN aproxima-se do centro da nuvem e a injeção de energia do choque do RSN na nuvem termina, $\gamma=\pi / 2$ e $v_{c s}=0$. Notamos que somente para um choque plano, $\gamma$ será igual a $\pi / 2$ quando o choque atinge exatamente o centro da nuvem. Para $r_{c} \leq R_{R S N}$, $\gamma$ será $=\pi / 2$ um pouco antes de atingir o centro. O valor médio da velocidade integrada sobre o tempo de cruzamento do RSN é

$$
\hat{v}_{c s} \simeq v_{R S N}\left(\frac{n_{s h}}{n_{c}}\right)^{0.5} \frac{2}{t_{c, R S N}} \int_{0}^{t_{c, R S N}} \cos \gamma(t) d t
$$

onde

$$
\cos \gamma(t)=\frac{d^{2}-R_{R S N}^{2}(t)-r_{c}^{2}}{2 R_{R S N}(t) r_{c}}
$$

$d$ é a distância entre o centro do RSN e o centro da nuvem, $R_{R S N}$ é o raio do RSN, $r_{c}$ é o raio da nuvem, $n_{c}$ é a densidade inicial da nuvem, $n_{s h}$ é a densidade da shell do RSN e 


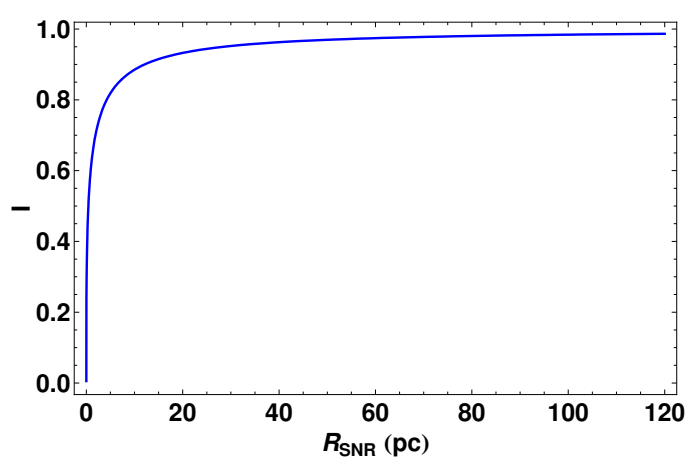

Figura 2.3: Valores de I para uma nuvem com $r_{c}=10 \mathrm{pc}$ e para diferentes valores de $R_{R S N}$

$v_{R S N}$ é a velocidade de expansão do RSN.

Assumimos que a distância entre o centro da nuvem e o centro do RSN permanece constante:

$$
d=R_{R S N}+r_{c}
$$

Após um tempo $t_{c, R S N}$, esta distância pode ser escrita como

$$
d=\sqrt{R_{R S N}(t)^{2}+r_{c}^{2}}
$$

Neste tempo o raio do RSN é aproximadamente:

$$
R_{R S N}(t) \simeq R_{R S N}+v_{R S N} t_{c, R S N}
$$

Assim:

$$
t_{c, R S N} \simeq \frac{\sqrt{R_{R S N}^{2}+2 r_{c} R_{R S N}}-R_{R S N}}{v_{R S N}}
$$

onde

$$
R_{R S N}=R_{R S N}(0)
$$

A Figura 2.3 apresenta o gráfico do termo integral da eq. (2.10),

$$
I=\frac{2}{t_{c, R S N}} \int_{0}^{t_{c, R S N}} \cos \gamma(t) d t
$$


para uma nuvem com $r_{c}=10$ pc como uma função de $R_{R S N}$. Este claramente mostra que o efeito da curvatura será relevante apenas para valores de $R_{R S N} / r_{c}$ próximos da unidade (Leão, 2007; Leão et al., 2009).

Agora, o fator de correção (I) deve ser multiplicado por $v_{c s}$ para o caso de interações nuvem-RSN obtendo-se:

$$
\hat{v}_{c s}=v_{R S N}\left(\frac{n_{s h}}{n_{c}}\right)^{1 / 2} I
$$

Encontramos então:

$$
\hat{v}_{c s, a} \sim 21 \frac{E_{51}^{1 / 2} I_{5}}{R_{R S N, 50}^{3 / 2} n_{c, 10}^{1 / 2}} \mathrm{~km} / \mathrm{s}
$$

no caso de um choque gerado por um RSN na fase adiabática onde $I_{5}$ é o fator I (eq. 2.10) calculado para $R_{R S N} / r_{c}=5, n_{c, 10}$ é a densidade da nuvem não-chocada em unidades de $10 \mathrm{~cm}^{-3}, E_{51}$ é a energia da SN em unidades de $10^{51}$ erg e $R_{R S N, 50}$ é o raio da 'shell' do RSN em unidades de 50 pc, e

$$
\hat{v}_{c s, r} \sim 23 \frac{E_{51}^{0.8} f_{10}^{1 / 2} I_{5}}{R_{R S N, 50}^{5 / 2} n_{c, 10}^{1 / 2} n^{0.41}} \mathrm{~km} / \mathrm{s}
$$

para um choque gerado por um RSN na fase radiativa, onde $f_{10}$ é o contraste de densidade entre a 'shell' do RSN e a densidade de MIS em unidades de 10. Com isso podemos calcular o tempo que a onda de choque leva para atravessar a nuvem (the cloud crushing time), $t_{c c}$ :

$$
t_{c c}=\frac{2 r_{c}}{v_{R S N} I}\left(\frac{\rho_{c}}{\rho_{R S N}}\right)^{1 / 2}=\frac{2 r_{c}}{\hat{v}_{c s}},
$$

No caso de uma nuvem não magnetizada encontramos (Melioli et al., 2006):

$$
t_{c c, a} \sim 4.7 \times 10^{5} \frac{n_{c, 10}^{0.5} r_{c, 10} R_{R S N, 50}^{1.5}}{I_{5} E_{51}^{0.5}} \text { anos }
$$

para um RSN na fase adiabática, onde $r_{c, 10}$ é o raio da nuvem em unidades de 10 pc. No caso de um RSN na fase radiativa teremos 


$$
t_{c c, r} \sim 4.3 \times 10^{5} \frac{r_{c, 10} R_{R S N, 50}^{2.5} n_{c, 10}^{0.5} n^{0.41}}{I_{5} f_{10}^{0.5} E_{51}^{0.8}} \text { anos }
$$

Depois que o choque atinge o centro da nuvem ele rebate e na ausência de efeitos de auto-gravidade, a nuvem comprimida começa a reexpandir. Teremos das equações acima um número de Mach para o choque propagante na nuvem não magnetizada dado por

$$
M=\frac{\hat{v}_{c s}}{\gamma^{1 / 2} c_{s}}
$$

onde $c_{s}=\left[\frac{k_{B} T_{c}}{\mu m_{H}}\right]^{1 / 2}$ é a velocidade do som na nuvem não chocada. Assim, para a interação com um RSN na fase adiabática, teremos

$$
M_{a} \approx 40.3 \frac{E_{51}^{0.5} I_{5}}{T_{c, 100}^{0.5} R_{R S N, 50}^{1.5} n_{c, 10}^{0.5}}
$$

onde $T_{c, 100}$ é a temperatura da nuvem em unidades de $100 \mathrm{~K}$, e

$$
M_{r} \approx 44.1 \frac{f_{10}^{0.5} E_{51}^{0.8} I_{5}}{n_{c, 10}^{0.5} T_{c, 100}^{0.5} R_{R S N, 50}^{2.5} n^{0.41}}
$$

para a interação ocorrendo com um RSN na fase radiativa, onde $n$ é a densidade do meio ambiente. A densidade da nuvem, $n_{c, s h}$, após a interação com o RSN pode ser encontrada usando as relações de Rankine-Hugoniot para um choque forte radiativo (Draine \& McKee, 1993). Como neste caso o tempo de resfriamento radiativo é bem menor do que as escalas de tempo dinâmicas do sistema podemos assumir que a onda de choque que se propaga dentro da nuvem é aproximadamente isotérmica para o gás chocado a temperaturas menores que $10^{4} \mathrm{~K}$. Temos então, para uma nuvem não magnetizada (Leão 2007):

$$
n_{c, s h, a} \sim \frac{1.6 \times 10^{4}}{R_{R S N, 50}^{3}} \frac{E_{51} I_{5}^{2}}{T_{c, 100}} \mathrm{~cm}^{-3}
$$

para o caso do remanescente de supernova na fase adiabática, e

$$
n_{c, s h, r} \sim \frac{1.9 \times 10^{4}}{R_{R S N, 50}^{5}} \frac{E_{51}^{1.6} I_{5}^{2} f_{10}}{T_{c, 100} n^{0.82}} \quad \mathrm{~cm}^{-3}
$$

para o caso do remanescente de supernovas na fase radiativa. 


\subsubsection{A inclusão do campo magnético na nuvem}

Como mencionado anteriormente (Cap. 1), medições Zeeman indicam que as razões massa-fluxo magnético em núcleos densos de nuvens moleculares são próximas ou pouco maiores do que o valor crítico (e.g., Crutcher 1999, 2005, 2008), enquanto que nuvens neutras difusas são subcríticas. Em outras palavras, o campo magnético médio típico destas nuvens $(\sim 6 \mu \mathrm{G}-10 \mu \mathrm{G})$ é maior do que o valor mínimo $\left(B_{c r} \simeq 2 \pi G^{1 / 2} \mathrm{~N}\right.$, onde $\mathrm{N}$ é a densidade superficial da nuvem) necessário para a pressão magnética equilibrar a gravidade, de modo que a nuvem isolada em principio não deve colapsar gravitacionalmente (Heiles \& Troland, 2005; Crutcher et al., 2010b). O impacto com um RSN irá comprimir o campo magnético atrás do choque aumentando-o ainda mais. Isto devera afetar a evolução do material chocado e portanto, suas condições para o colapso gravitacional. Em particular, o campo magnético tenderá a reduzir a força do impacto.

Vamos considerar uma nuvem difusa neutra magnetizada. Por simplicidade, vamos admitir que seu campo magnético é inicialmente uniforme e, para maximizar os efeitos do campo também assumiremos que este é inicialmente normal à velocidade de propagação do choque do RSN no momento do impacto. Os campos magnéticos devem estar distribuídos de forma randômica, o que significa que somente uma fração de sua força será de fato normal à velocidade do choque e trabalhará contra o impacto. Veremos a seguir que quando tomamos um valor efetivo para o campo magnético da nuvem $B_{c} \simeq 1 \mu \mathrm{G}$, seu efeito sobre os diagramas de formação estelar não é tão relevante. Contudo, quando escolhemos valores $\sim 5-10 \mu \mathrm{G}$, o domínio permitido para a formação estelar nos diagramas diminui consideravelmente.

As relações de Rankine-Hugoniot para um choque forte radiativo em presença de campo magnético (com $M \geq 10$ ) (veja por exemplo Draine \& McKee 1993), são

$$
\begin{gathered}
T_{c, s h}=T_{c} \\
n_{c, s h, B}=y n_{c}
\end{gathered}
$$




$$
\begin{gathered}
B_{c, s h}=y B_{c} \\
y=\frac{4}{2 M^{-2}+M_{A}^{-2}+\left[\left(2 M^{-2}+M_{A}^{-2}\right)^{2}+8 M_{A}^{-2}\right]^{1 / 2}}
\end{gathered}
$$

onde $T_{c, s h}, n_{c, s h, B}$ e $B_{c, s h}$ são a temperatura, densidade e o campo magnético do gás da nuvem chocada, respectivamente, $M$ é o número de Mach dado pelas Eqs. (2.22) e (2.23) para um RSN na fase adiabática e para um na fase radiativa, respectivamente, e $M_{A}$ é o número de Mach Afvénico. Para uma interação com um RSN na fase adiabática, usando a equação $(2.16), M_{A}$ torna-se

$$
M_{A, a}=\frac{\hat{v}_{c s}}{v_{A}}=68.5 \frac{E_{51}^{1 / 2} I_{5}}{B_{c, 6} R_{R S N, 50}^{3 / 2}}
$$

onde $v_{A}$ é a velocidade de Alfvén na nuvem, $B_{c, 6}$ é o campo magnético da nuvem antes do choque em unidades de $10^{-6} G$ e $I_{5}$ é o fator I (eq. 2.10) calculado para $R_{R S N} / r_{c}=5$. Para uma interação com um RSN na fase radiativa, usando Eq. (2.17), $M_{A}$ é dado por:

$$
M_{A, r}=75 \frac{f_{10}^{1 / 2} E_{51}^{0.8} I_{5}}{B_{c, 6} R_{R S N, 50}^{5 / 2} n^{0.41}}
$$

Substituindo (2.30) e (2.22) na Eq. (2.27), obtemos a densidade do gás chocado na nuvem magnetizada após a interação com o RSN adiabático:

$$
n_{c, s h, B, a} \sim \frac{4 \times 10^{4} E_{51} n_{c, 10} I_{5}^{2}}{R_{R S N, 50}^{3}\left[F+\sqrt{F^{2}+G}\right]}
$$

onde

$$
F=1.2 T_{c, 100} n_{c, 10}+0.21 B_{c, 6}^{2}
$$

$\mathrm{e}$

$$
G=1700 \frac{B_{c, 6}^{2} E_{51} I_{5}^{2}}{R_{R S N, 50}^{3}}
$$

Usando (2.31) e (2.23), obtemos a densidade do gás chocado na nuvem após a interação com um RSN na fase radiativa: 


$$
n_{c, s h, B, r} \sim \frac{4 \times 10^{4} E_{51}^{1.6} n_{c, 10} f_{10} I_{5}^{2}}{R_{R S N, 50}^{5} n^{0.82}\left[H+\sqrt{H^{2}+J}\right]}
$$

onde

$$
H=1 T_{c, 100} n_{c, 10}+0.18 B_{c, 6}^{2}
$$

$\mathrm{e}$

$$
J=1430 \frac{B_{c, 6}^{2} E_{51}^{1.6} f_{10} I_{5}^{2}}{R_{R S N, 50}^{5} n^{0.82}}
$$

Quando $1 \ll M_{A} \ll M^{2}$, as equações acima tornam-se (e.g., Leão 2007):

$$
n_{c, s h, a}=2^{1 / 2} M_{A} n_{c} \sim 970 n_{c, 10} \frac{E_{51}^{1 / 2} I_{5}}{B_{c, 6} R_{R S N, 50}^{3 / 2}} \mathrm{~cm}^{-3}
$$

$\mathrm{e}$

$$
n_{c, s h, r}=2^{1 / 2} M_{A} n_{c} \sim 1060 n_{c, 10} \frac{f_{10}^{1 / 2} E_{51}^{0.8} I_{5}}{B_{c, 6} R_{R S N, 50}^{5 / 2} n^{0.41}} \mathrm{~cm}^{-3}
$$

No limite $1 \ll M^{2} \ll M_{A}$, o que implica um campo magnético dinamicamente desprezível atrás do choque, recuperamos as equações sem campo magnético apresentadas na secção anterior.

\subsection{Condições para indução do colapso gravitacional na nuvem}

Podemos agora derivar as condições para a indução da instabilidade gravitacional na nuvem devido à interação com um RSN. Um primeiro vínculo determinará o limite da massa de Jeans para o material comprimido da nuvem. Um segundo estabelecerá a condição na qual a frente de choque do RSN não será forte o suficiente para destruir a nuvem completamente (e fazer com que o gás disperse no meio interestelar antes de se tornar gravitacionalmente instável). O terceiro vínculo estabelecerá a extensão de penetração da frente de choque antes de perder sua energia. O choque deve ter energia suficiente para comprimir tanto material da nuvem quanto possível antes de enfraquecer. Nas seções seguinte derivaremos matematicamente estes vínculos (Leão et al., 2009). 


\subsubsection{O Vínculo da Massa de Jeans}

\section{$\mathrm{Na}$ ausência de campo magnético}

Melioli et al. (2006) derivaram o limite sobre a massa de Jeans para o gás chocado em interações envolvendo nuvens não magnetizadas. Com a correção realizada no cálculo do fator I obtivemos novas aproximações para as constantes multiplicativas. Quando o RSN interagente está ainda no regime adiabático a massa de Jeans do material chocado da nuvem na ausência de campo magnético é dada por:

$$
m_{J, a} \sim 750 \frac{T_{c, 100}^{2} R_{R S N, 50}^{1.5}}{I_{5} E_{51}^{0.5}} \mathrm{M}_{\odot}
$$

e se o RSN esta no regime radiativo:

$$
m_{J, r} \sim 685 \frac{T_{c, 100}^{2} R_{R S N, 50}^{2.5} n^{0.41}}{I_{5} f_{10}^{0.5} E_{51}^{0.8}} \mathrm{M}_{\odot}
$$

Em termos do raio do RSN, as condições acima implicam em:

$$
R_{R S N, a} \lesssim 55.8 \frac{E_{51}^{1 / 3} I_{5}^{2 / 3} n_{c, 10}^{2 / 3} r_{c, 10}^{2}}{T_{c, 100}^{4 / 3}} \mathrm{pc}
$$

e

$$
R_{R S N, r} \lesssim 55.1 \frac{E_{51}^{0.33} I_{5}^{0.4} f_{10}^{0.2} n_{c, 10}^{0.4} r_{c, 10}^{2.4}}{T_{c, 100}^{0.8} n^{0.17}} \mathrm{pc}
$$

respectivamente.

\section{Em presença de campo magnético}

Quando incluímos o campo magnético na nuvem a massa mínima correspondente (massa de Jeans) que o material chocado deve ter de modo a sofrer colapso gravitacional é dada, usando o Teorema do Virial, por

$$
m_{J, B} \simeq \frac{6.63 \times 10^{24}}{n_{c, s h}^{1 / 2}}\left[\frac{B_{c, s h}^{2}}{8 \pi n_{c, s h}}+3 k_{B} T_{c, s h}\right]^{3 / 2} \mathrm{M}_{\odot}
$$


Em termos dos parâmetros da nuvem antes do choque (condições iniciais) e do RSN esta condição fica (usando as Eqs. 2.26 - 2.28):

$$
m_{J, B} \simeq 2100\left[\frac{4 y B_{c, 6}^{2}}{n_{c, 10}}+4.14 T_{c, 100}\right]^{3 / 2} \frac{1}{\left(y n_{c, 10}\right)^{1 / 2}} \mathrm{M}_{\odot}
$$

Podemos obter $R_{R S N}$ como uma função de $n_{c}$ e assim obter uma condição aproximada para o colapso gravitacional resolvendo a condição $m_{c} \geq m_{J, B}$, onde $m_{c}$ é a massa da nuvem, ou:

$$
n_{c, 10}^{2} r_{c, 10}^{2} y^{1 / 3} \geq 5.63 n_{c, 10} T_{c, 100}+0.54 B_{c, 6}^{2} y
$$

onde $y$ é dado pela Eq. (2.29). Substituindo y na equação acima e usando as Eqs. (2.22) e (2.30) obtemos numericamente o limite de Jeans, ou um limite superior para $R_{R S N, a}$, para o gás chocado de uma nuvem magnetizada devido ao impacto com um RSN no regime adiabático e se substituirmos (2.23) e (2.31) obtemos as mesmas condições para uma interação com um RSN no regime radiativo.

Notamos que no limite em que $\frac{B^{2}}{8 \pi} \ll \rho c_{s}^{2}$ a equação acima (2.42) recupera as soluções (2.38) e (2.39), para as fases adiabática e radiativa, respectivamente.

\subsubsection{Vínculo para a não-destruição da nuvem devido ao im- pacto de um RSN}

\section{Na ausência de campo magnético}

Como observamos anteriormente se a interação RSN-nuvem é muito forte esta pode levar à completa destruição da nuvem mesmo se o material chocado tiver a priori uma massa total superior ao limite de Jeans. A estabilidade de uma nuvem contra a destruição logo após o impacto de um vento ou um RSN devido ao crescimento das instabilidades Rayleigh-Taylor e Kelvin-Helmholtz foram exploradas por vários autores (veja por exemplo Murray et al. 1993; Poludnenko et al. 2002; Melioli, de Gouveia Dal Pino \& Raga 2005, e referências nestes). Para obtermos as condições para que a nuvem chocada não seja destruída devemos comparar a escala de tempo de queda-livre gravitacional do 
gás chocado com a escala de tempo de destruição devido ao impacto e consequente desenvolvimento destas instabilidades, $t_{d}$. Mais precisamente, para termos o colapso, um modo gravitacionalmente instável (com tempo típico $t_{u n}$ ) deve crescer rápido o bastante para tornar-se não linear dentro da escala de tempo da interação nuvem-choque do RSN (veja por exemplo Nakamura et al. 2006). Seguindo Nakamura et al. a condição para a não destruição fica: $t_{u n}<t_{d}$, onde $t_{d}$ é umas poucas vezes o tempo de cruzamento, $t_{c c}=2 r_{c} / \hat{v}_{c s}$ (eq. 2.19 e 2.20), isto é, o tempo que o choque interno leva para cruzar a nuvem. Com a ajuda de simulações numéricas que levam em conta os efeitos do resfriamento radiativo fora do equilíbrio nas interações entre nuvens e RSN, Melioli, de Gouveia Dal Pino \& Raga (2005) mostraram que o tempo de destruição da nuvem é $t_{d} \sim 4-6 t_{c c}$, quando o resfriamento radiativo está presente. Portanto para termos o colapso esperamos que $t_{u n} \lesssim 5 t_{c c}$. Isto implica um número de Mach:

$$
M \lesssim 60.5 \frac{\mu_{0}^{7 / 9}}{\zeta^{2}} .
$$

onde $\zeta \approx 0.8$ é o fator de compressão do choque na direção transversa (Nakamura et al. 2006) e

$$
\mu_{0}=m_{c} \frac{\left(G^{3} \rho_{c}\right)^{1 / 2}}{c_{s}^{3}}
$$

é a massa adimensional da nuvem, e $m_{c}$ é a massa da nuvem. Assim temos:

$$
M \lesssim 47\left(\frac{n_{c, 10}}{T_{c, 100}}\right)^{1.16} r_{c, 10}^{2.3}
$$

Esta relação implica:

$$
R_{R S N, a} \gtrsim 45 \frac{E_{51}^{0.33} T_{c, 100}^{0.44} I_{5}^{0.66}}{n_{c, 10} r_{c, 10}^{1.56}} \mathrm{pc}
$$

para uma interação com um RSN no regime adiabático, e

$$
R_{R S N, r} \gtrsim 48.7 \frac{E_{51}^{0.33} f_{10}^{0.2} T_{c, 100}^{0.26} I_{5}^{0.4}}{n_{c, 10}^{0.7} n^{0.17} r_{c, 10}^{0.93}} \quad \mathrm{pc}
$$

para uma interação com um RSN na fase radiativa. 


\section{Na presença de campo magnético}

Quando a nuvem é magnetizada tendo um campo magnético médio normal à velocidade do choque a força deste deve diminuir devido à pressão magnética e forças de tensão. Usando a mesma condição para o colapso descritas acima, porém agora incluindo o campo magnético na nuvem, obtemos a seguinte condição sobre o número de Mach:

$$
\frac{M[1+y /(3 \beta)]^{3 / 8}}{y^{5 / 8}} \lesssim 18.7\left(\frac{n_{c, 10}}{T_{c, 100}}\right)^{7 / 8} r_{c, 10}^{7 / 4}
$$

onde

$$
\beta=\frac{8 \pi \rho_{c} c_{s}^{2}}{B_{c}^{2}}
$$

Substituindo as relações encontradas para $y$ (Eq. 2.29), $M$ e $M_{A}$ para uma colisão com um RSN adiabático (Eqs. 2.30 e 2.22) e com um radiativo (Eqs. 2.31 e 2.23) na equação acima (Eq. 2.48) podemos encontrar numéricamente novos vínculos para $R_{R S N, a}$ e $R_{R S N, r}$, respectivamente, de modo a não destruir a nuvem magnetizada no impacto e permitir o colapso gravitacional.

\subsubsection{Vínculo da extensão da penetração da frente de choque do RSN na nuvem}

Além dos vínculos derivados nas secções anteriores, ainda precisamos de uma terceira condição para o impacto. Como comentado anteriormente, o choque deve ter força suficiente para penetrar na nuvem e comprimir tanto material quanto possível para que este sofra colapso gravitacional.

\section{Na ausência de campo magnético}

Quando o choque propaga-se dentro da nuvem ele irá desacelerar devido às contínuas perdas radiativas e à resistência do material não chocado da nuvem. Podemos estimar o tempo aproximado no qual o choque irá parar dentro da nuvem $\left(t_{s t}\right)$ usando argumentos de conservação de energia. Em $t=t_{s t}$, a velocidade do gás chocado na nuvem torna-se 
$\approx c_{s}$, de modo que o número de Mach irá decair de seu valor inicial logo após o impacto para $M \sim 1$. Da conservação de energia atrás do choque encontramos aproximadamente que:

$$
\begin{gathered}
\frac{5}{2} \frac{k_{B} T_{c, s h}(0)}{\mu m_{H}}+\frac{1}{2} v_{s h}^{2}(0) \\
\simeq \frac{5}{2} \frac{k_{B} T_{c, s h}\left(t_{s t}\right)}{\mu m_{H}}+\frac{1}{2} v_{s h}^{2}\left(t_{s t}\right)+\frac{\Lambda\left[T_{c, s h}\left(t_{s t}\right)\right] n_{c, s h}\left(t_{s t}\right)}{\mu m_{H}} t_{s t}
\end{gathered}
$$

onde o lado esquerdo é a energia total atrás do choque na nuvem imediatamente após o impacto e o lado direito é a energia total atrás do choque no tempo $t_{s t}$ quando este pára. A temperatura e a densidade inicial do material chocado da nuvem no lado esquerdo da equação são aproximadamente dados pelos valores adiabáticos. No lado direito, em $t_{s t}$, a temperatura e a densidade do material chocado são obtidos a partir das relações de Rankine-Hugoniot para um choque radiativo com $M \simeq 1$, ou seja, $T_{c, s h}\left(t_{s t}\right) \simeq T_{c}$, and $n_{c, s h}\left(t_{s t}\right) \simeq n_{c}$. A função de resfriamento radiativo em $t_{s t}, \Lambda\left[T_{s h}\left(t_{s t}\right)\right] \simeq \Lambda\left(T_{c}\right)$, pode ser aproximada para um gás oticamente fino (veja Dalgarno \& McCray 1972). A substituição destas condições na equação acima resulta:

$$
t_{s t} \simeq \frac{31}{32} \frac{\mu m_{H}}{n_{c} \Lambda\left(T_{c}\right)} \hat{v}_{c s}^{2} \mathrm{~s} .
$$

onde $\hat{v}_{c s}^{2}$ é dado pela eq. (2.15) ou, em termos do número de Mach inicial, pelas eq. (2.22) e (2.23), e onde $\Lambda\left(T_{c}\right)$ é calculado para $T_{c}=100 \mathrm{~K}$.

Usamos este tempo para calcular a distância máxima que a frente de choque (iniciada pelo RSN) pode viajar dentro da nuvem antes de ser freada. Esta distância é então comparada com o diâmetro da nuvem $\left(\hat{v}_{c s} t_{s t} \geq 2 r_{c}\right)$ de forma a estabelecer o tamanho máximo (a energia mínima) que o RSN deve ter para gerar um choque capaz de comprimir a nuvem tanto quanto possível antes de parar. No caso de um RSN adiabático temos:

$$
R_{R S N, a} \lesssim 170 \frac{E_{51}^{0.33} I_{5}^{0.66}}{\left(r_{c, 10} \Lambda_{27}\right)^{2 / 9} n_{c}^{0.5}} \quad \mathrm{pc}
$$


E no caso de um RSN no regime radiativo encontramos:

$$
R_{R S N, r} \lesssim 108 \frac{E_{51}^{0.32} I_{5}^{2 / 5} f_{10}^{1 / 5}}{n_{c, 10}^{1 / 3} n^{0.16}\left(r_{c, 10} \Lambda_{27}\right)^{2 / 15}} \mathrm{pc}
$$

onde $\Lambda_{27}$ é a função de resfriamento $(\Lambda)$ em unidades de $10^{-27}$ erg. $\mathrm{cm}^{3} \mathrm{~s}^{-1}$, que é o valor aproximado para $T_{c}=100 \mathrm{~K}$ e uma fração de ionização $10^{-4}$.

\section{Na presença de campo magnético}

Quando consideramos a presença de um campo magnético na nuvem normal à velocidade do choque nossas estimativas para a conservação do choque implicam aproximadamente em:

$$
\begin{gathered}
\frac{5}{2} \frac{k_{B} T_{c, s h}(0)}{\mu m_{H}}+\frac{1}{2} v_{s h}^{2}(0)+\frac{B_{c, s h}^{2}(0)}{4 \pi \mu m_{H} n_{c, s h}(0)} \\
\simeq \frac{5}{2} \frac{k_{B} T_{c, s h}\left(t_{s t}\right)}{\mu m_{H}}+\frac{1}{2} v_{s h}^{2}\left(t_{s t}\right)+\frac{\Lambda\left[T_{c, s h}\left(t_{s t}\right)\right] n_{c, s h}\left(t_{s t}\right)}{\mu m_{H}} t_{s t} \\
+\frac{B_{c, s h}\left(t_{s t}\right)^{2}}{4 \pi \mu m_{H} n_{c, s h}\left(t_{s t}\right)}
\end{gathered}
$$

Onde novamente o lado esquerdo dá a energia total atrás do choque na nuvem imediatamente após o impacto com a temperatura, densidade e campo magnético iniciais do material chocado da nuvem sendo aproximadamente dados pelos valores adiabáticos. O lado direito dá a energia total atrás do choque total em $t_{s t}$ quando $v_{s h}$ diminui para $v_{s h}\left(t_{s t}\right)^{2} \simeq \gamma c_{s}^{2}+v_{A}^{2}$. Neste tempo, $n_{c, s h}\left(t_{s t}\right), T_{c, s h}\left(t_{s t}\right)$, e $B_{c, s h}\left(t_{s t}\right)$ são obtidos a partir das relações de Rankine-Hugoniot para um choque radiativo magnetizado (eqs. 2.26 a 2.28), para $y \simeq 1$, de modo que novamente $\Lambda\left[T_{s h}\left(t_{s t}\right)\right] \simeq \Lambda\left(T_{c}\right)$. A substituição destas condições na equação acima resulta aproximadamente na mesma estimativa para $t_{s t}$ que na Eq. (2.50) para o caso não magnetizado e, portanto, os mesmos limites superiores $R_{R S N}$ de forma que o choque varra a nuvem tanto quanto possível antes de ser parado 
(Eqs. 2.51 e 2.52). Isto é devido ao fato de que para os campos observados nestas nuvens $\left(\sim 10^{-4}-10^{-6} G\right)$, os termos de energia magnética na Eq. (2.53) são desprezíveis quando comparados aos outros.

No próximo capítulo, agruparemos esses vínculos em diagramas que determinarão o espaço paramétrico no qual a formação estelar induzida por interações entre RSNs e nuvens interestelares é viável ou não. Veremos que esse espaço é bem limitado. Derivaremos também a eficiência de formação estelar devida a esse processo. 


\title{
Capítulo 3
}

\section{Formação Estelar induzida por Choques de SNs - Aplicações a Nuvens Difusas Frias}

\author{
"For my part, I know nothing with any certainty, \\ but the sight of the stars makes me dream in the same simple way \\ as I dream about the black dots representing towns and villages on a map." \\ (Vincent van Gogh)
}

No Capítulo anterior, considerando o impacto de um remanescente de uma supernova (RSN) com uma nuvem neutra magnetizada derivamos quais as condições favoráveis para a indução de instabilidade gravitacional na nuvem e à formação de estrelas. Neste Capítulo, usando esses vínculos, construiremos diagramas do raio do RSN, $R_{R S N}$, versus a densidade inicial da nuvem, $n_{c}$, que delimitam um domínio no espaço de parâmetros onde a formação estelar é permitida. Este trabalho é uma extensão de um estudo anterior realizado sem se considerar a presença de campos magnéticos e aplicado especificamente à região de formação estelar de beta pictoris. (Melioli et al. 2006). Os resultados preliminares da análise a seguir foram ainda apresentados na dissertação de mestrado de Leão (2007). Os diagramas foram também testados com simulações tridimensionais (3D) magnetohidrodinâmicas (MHD) com resfriamento radiativo envolvendo um RSN e uma nuvem 
auto-gravitante, como descreveremos a seguir, e verificamos que a análise numérica é consistente com os resultados previstos pelos diagramas. Enquanto a inclusão de um campo magnético homogêneo aproximadamente perpendicular à velocidade de impacto do RSN com uma intensidade $\sim 1 \mu \mathrm{G}$ dentro da nuvem resulta somente em uma pequena diminuição do domínio paramétrico de formação estelar no diagrama relativo ao sem campo magnético, um campo magnético mais forte $(\sim 10 \mu \mathrm{G})$ causa uma diminuição significativa, como esperado. Embora derivados de considerações analíticas simples estes diagramas fornecem uma ferramenta útil para identificar locais onde a formação estelar poderia ser desencadeada pelo impacto de uma onda de choque de uma supernova (SN). Aplicações destes a algumas regiões de nossa Galáxia (por exemplo, a grande concha de CO na direção de Cassiopeia, e a Nuvem Periférica 2 na direção da constelação de Escorpião) revelaram que a formação de estrelas nestes locais poderiam ter sido induzidas por ondas de choque de RSNs para valores específicos da densidade inicial da nuvem neutra e do raio do RSN. Finalmente, avaliamos a eficiência de formação estelar (sfe) para este tipo de interação e encontramos que é geralmente menor do que os valores observados em nossa Galáxia (sfe $\sim 0.01-0.3$ ). Este resultado sugere que o mecanismo investigado, embora poderoso para induzir a formação de estruturas, turbulência supersônica e eventualmente, formação estelar local, não parece ser suficiente para impelir a formação estelar global em galáxias normais com formação estelar, nem mesmo quando o campo magnético nas nuvens neutras é desprezado. Os resultados aqui reportados foram publicados em Leão et al. 2009 (veja cópia do artigo no Apêndice C)

\subsection{Diagramas para a Formação Estelar}

Os três vínculos derivados nas Secções 2.3.1, 2.3.2, e 2.3.3 do Capítulo 2 para nuvens não magnetizadas e magnetizadas interagindo com RSNs na fase adiabática ou na radiativa destas são graficados juntos em um diagrama do raio do RSN em função da densidade inicial da nuvem (antes de ser chocada) para diferentes valores de seu raio. As Figuras 3.1 e 3.2 mostram exemplos destes diagramas para nuvens não magnetizadas e magnetizadas com $B=1 \mu \mathrm{G}$, respectivamente, com uma temperatura inicial $T_{c}=100 \mathrm{~K}$ e raio 
variando entre $r_{c}=1$ pc e 20 pc. ${ }^{1}$ Os três vínculos delimitam uma região sombreada no espaço de parâmetros onde as condições são apropriadas para o colapso gravitacional do material chocado da nuvem. Somente interações nuvem-RSN com condições físicas $\left(r_{c}\right.$, $n_{c}$, and $R_{R S N}$ ) situadas dentro da região sombreada (entre as linhas sólida, pontilhada e tracejada) podem levar à formação estelar. Escolhemos um remanescente de supernova na fase adiabática nestes diagramas, pois possui mais energia do que um com as mesmas características na fase radiativa (veja contudo na Figura $3.11 \mathrm{um}$ exemplo de uma interação envolvendo um RSN radiativo). Devemos notar que de acordo com as equações $(2.38,2.39,2.46,2.47,2.51$ e 2.52), os vínculos não são muito sensíveis à temperatura inicial da nuvem e isto explica porque escolhemos um único valor característico. Notamos posteriormente que uma nuvem com uma temperatura entre $10-50 \mathrm{~K}$ e um raio maior que 10 pc já é gravitacionalmente instável para uma grande faixa de densidades ( > 5 $\mathrm{cm}^{-3}$ quando o campo magnético é desprezado) e não requer em princípio, uma onda de choque para induzir a formação estelar. Além disso, será mais difícil para uma frente de choque de um RSN destruir uma nuvem nestas temperaturas.

A Figura 3.1 descreve interações com uma nuvem não magnetizada, como apresentado em Melioli et al. 2006. Contudo as modificações descritas nas seções 2.3.1-2.3.3 no Capítulo anterior resultaram em modificações nos diagramas. Seguem algumas observações em relação a esta Figura:

1. No artigo de Melioli et al. (2006) as curvas pontilhadas (azuis) do diagrama foram construídas para apenas um valor da função de resfriamento radiativo do material chocado, isto é $\Lambda\left(T_{s h}\right) \simeq 10^{-27} \mathrm{erg} \mathrm{cm}^{3} \mathrm{~s}^{-1}$ o qual é válido para um gás difuso com temperatura $100 \mathrm{~K}$ e fração de ionização $\leq 10^{-4}$. Considerando que o vínculo estabelecido pela curva pontilhada (azul) é sensível a $\Lambda\left(T_{s h}\right)$ (através da Eq. 2.51) que por sua vez pode variar por duas ordens de magnitude dependendo do valor da fração de ionização do gás da nuvem, graficamos nos diagramas três diferentes

\footnotetext{
${ }^{1}$ Notamos que nos diagramas das Figs. 3.1 e 3.2, e na maioria dos diagramas deste trabalho, adotamos uma densidade ambiente $n=0.05 \mathrm{~cm}^{-3}$. Escolhemos esta baixa densidade de forma a tentar reproduzir da melhor forma o meio quente de baixa densidade mais comumente encontrado ao redor de um RSN, principalmente em sua fase adiabática.
} 

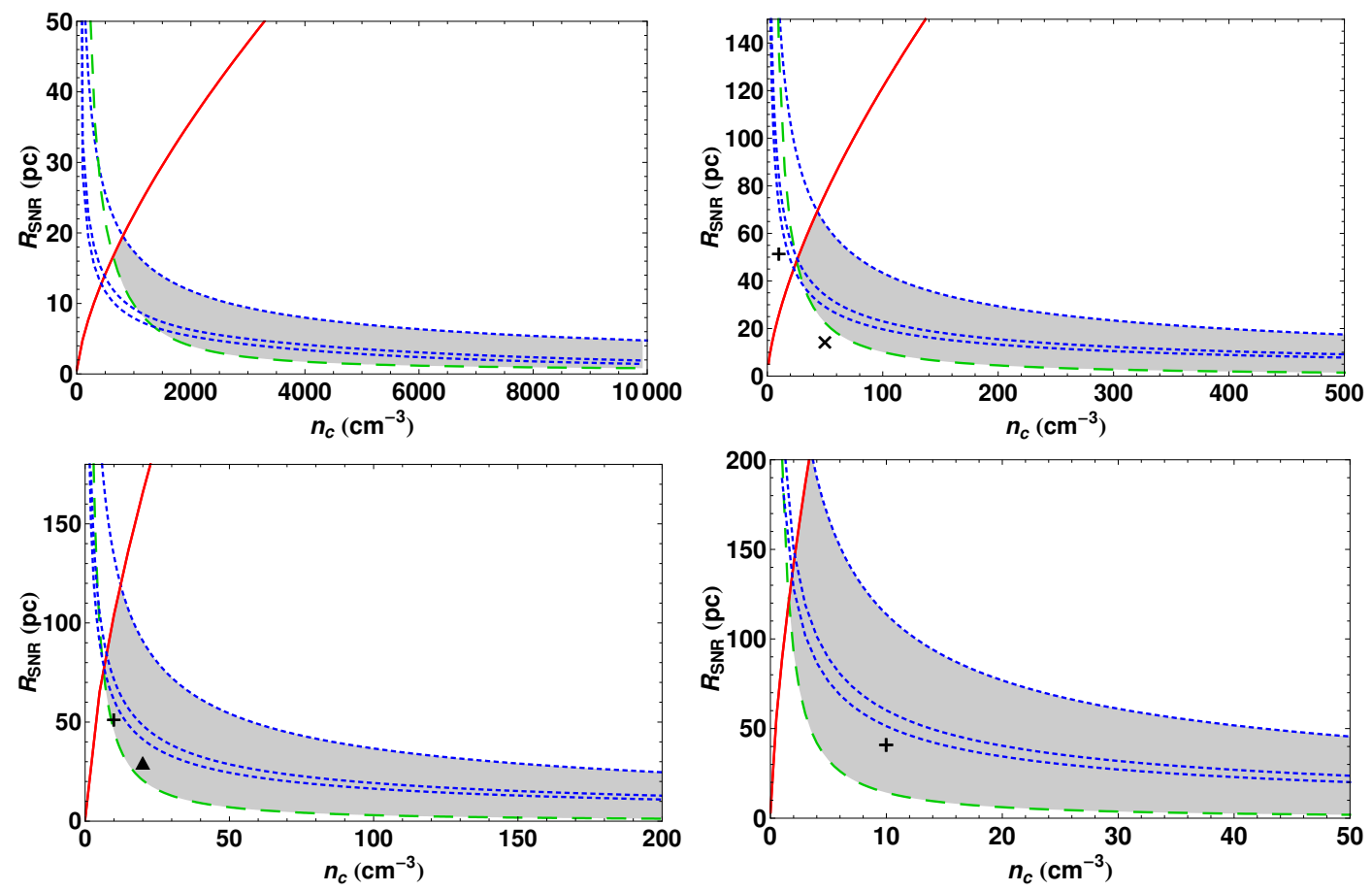

Figura 3.1: Vínculos para o raio do RSN versus a densidade inicial da nuvem para 4 diferentes raios de nuvens. Painéis superiores: à esquerda, nuvem com $r_{c}=1$ pc; à direita, $r_{c}=5$ pc. Painéis inferiores: à esquerda, nuvem com $r_{c}=10 \mathrm{pc}$; à direita, $r_{c}=20 \mathrm{pc}$. Linha tracejada (verde): limite superior para a completa destruição da nuvem após um encontro com um RSN adiabático derivado da Eq. (2.46); linha sólida (vermelha): limite superior para a nuvem chocada atingir a massa de Jeans derivado da Eq. (2.38); linha pontilhada (azul): limite superior para a frente de choque viajar dentro da nuvem antes de ser desacelerada a velocidades subsônicas derivado da Eq. (2.51) para diferentes valores da função de resfriamento $\Lambda\left(T_{s h}\left(t_{s t}\right)\right)=10^{-25} \mathrm{erg} \mathrm{cm}^{3} \mathrm{~s}^{-1}$ (curva inferior), $5 \times 10^{-26} \mathrm{erg}$ $\mathrm{cm}^{3} \mathrm{~s}^{-1}$ (curva central), e $3 \times 10^{-27} \mathrm{erg} \mathrm{cm}^{3} \mathrm{~s}^{-1}$ (curva superior). A área sombreada (entre as linhas sólida, tracejada e pontilhada) define a região onde a formação estelar pode ser induzida pela interação RSN-nuvem. As cruzes e o triângulo nas imagens indicam as condições iniciais assumidas para as nuvens nas simulações numéricas descritas na Secção 4.2 de Melioli et al. (2006).

curvas pontilhadas de modo a cobrir uma faixa mais ampla de possíveis frações de ionização de 0.1 a $10^{-4}$, correspondendo a $\Lambda\left(T_{s h}\right)=10^{-25} \mathrm{erg}^{\mathrm{cm}^{3}} \mathrm{~s}^{-1}$ (curva 
pontilhada inferior), $5 \times 10^{-26} \mathrm{erg} \mathrm{cm}^{3} \mathrm{~s}^{-1}$ (curva pontilhada central), e $3 \times 10^{-27}$ $\operatorname{erg} \mathrm{cm}^{3} \mathrm{~s}^{-1}$ (curva pontilhada superior), respectivamente (veja Dalgarno \& McCray 1972). A curva central corresponde ao valor médio de $\Lambda, 5 \times 10^{-26} \mathrm{erg} \mathrm{cm}^{3} \mathrm{~s}^{-1}$, e pode ser tomada como referência.

2. Na solução apresentada em Melioli et al. (2006) para a nuvem com $r_{c}=1$ pc, não há região sombreada onde a formação estelar é permitida. De acordo com as atuais correções e modificações, vemos que uma região de formação estelar fina sombreada aparece agora que a função de resfriamento $\Lambda$ possui/apresenta valores menores do que $10^{-25}$ erg $\mathrm{cm}^{3} \mathrm{~s}^{-1}$, ou frações de ionização $\lesssim 10^{-1}$ (painel superior esquerdo da Figura 3.1).

3. A cruz no painel inferior direito da Figura 3.1 para uma nuvem difusa com $r_{c}=$ 20 pc corresponde às condições iniciais da simulação numérica apresentada na Figura 6 do artigo de Melioli et al. (isto é, para um RSN a uma distância $R_{R S N} \sim$ 42 pc da superfície da nuvem). Naquele artigo a cruz encontra-se fora da região sombreada gravitacionalmente instável, logo acima do limite para uma penetração completa do choque dentro da nuvem (isto é, acima da linha pontilhada central azul no diagrama). Com as presentes modificações a cruz fica próxima ao limite superior da região sombreada instável para valores da função de resfriamento $\Lambda \lesssim 10^{-25}$ erg $\mathrm{cm}^{3} \mathrm{~s}^{-1}$, ou fração de ionização $\lesssim 10^{-1}$. Este resultado mostra quão sensível os diagramas analíticos são à escolha de $\Lambda$ para uma dada temperatura inicial da nuvem. De acordo com as simulações quimio-hidrodinâmicas com resfriamento radiativo apresentadas na Figura 6 do artigo de Melioli et al. (que corresponde à cruz no diagrama da Fig. 3.1), a frente de choque do RSN se desacelera antes de cruzar a nuvem completamente e o material chocado da nuvem não atinge as condições para se tornar gravitacionalmente instável, porém a shell fria e densa que se desenvolve pode se fragmentar e posteriormente gerar núcleos densos. Isto mostra uma ambiguidade nos resultados devido à sua sensibilidade à $\Lambda$ e à fração de ionização real do gás. Devemos também observar que o vínculo estabelecido pelas curvas pontilhadas (azuis) nos diagramas é de fato somente um limite superior para a condição de pe- 
netração do choque na nuvem. A condição de que o número de Mach do material chocado vai para $M \sim 1$ implica o equilíbrio de pressão entre o material chocado e o não chocado da nuvem no tempo $t_{s t}$ quando o choque pára dentro da nuvem. Um exame rápido das simulações numéricas da Figura 6 de Melioli et al. (2006), contudo, mostra que a frente de choque pára mesmo antes deste balanço ser atingido. Isto implica que o tempo $t_{s t}$ pode ser menor e portanto as curvas para este vínculo deveriam estar abaixo da posição prevista pela Eq. (2.51).

Apesar das importantes alterações acima nos diagramas da Figura 3.1, os principais resultados e conclusões do artigo de Melioli et al. (2006) para interações envolvendo RSNs com nuvens não magnetizadas, particularmente aqueles relacionados ao sistema estelar jovem de $\beta$-Pictoris, permanecem inalterados (veja abaixo contudo, as implicações para este sistema quando o campo magnético é incorporado à nuvem).

A Figura 3.2 mostra os mesmos diagramas que a Figura 3.1 exceto que agora estão inclusos os efeitos do campo magnético na nuvem, como descrito pelas Eqs. 2.42, 2.48 e 2.51. Notamos que a presença de um campo magnético normal à frente de choque com uma intensidade de $1 \mu \mathrm{G}$ diminui levemente o domínio de formação estelar, como esperado. O campo magnético possui um papel dominante sobre o vínculo da massa de Jeans (linha sólida vermelha nos diagramas) o que causa um deslocamento da região de formação estelar permitida para a direita (isto é, para densidades iniciais da nuvem maiores) quando comparado com os diagramas sem campo magnético da Fig. 3.1. Este deslocamento contudo deve ser interpretado com cuidado. Quando derivamos o vínculo de jeans em presença de uma campo magnético uniforme não nulo normal à frente de choque (eq. 2.40) assumimos um colapso unidimensional. Contudo, embora a presença de $\vec{B}$ deva afetar a compressão inicial e o colapso do material chocado, a evolução posterior e o colapso deste material em um espaço tridimensional ocorrerá principalmente na direção paralela a $\vec{B}$ e assim não será afetado por ele. Por esta razão devemos esperar que o domínio real de uma nuvem magnetizada instável nos diagramas da Figure 2 seja mais estendido para à esquerda da linha sólida (magenta) e será em ultimo caso delimitado pela linha ponto-tracejada vermelha que dá o vínculo de Jeans para um campo magnético 
nulo ou paralelo, como na Figura 3.1. Esta parte dos diagramas com $\vec{B}$ não nulo será particularmente importante para compará-los com as simulações numéricas tridimensionais das interações nuvem-RSN. Quando consideramos campos magnéticos maiores (5-10 $\mu \mathrm{G})$ existe um encolhimento significativo da região permitida para a formação estelar nos diagramas (veja a Figura 3.3). Podemos entender isto em termos da razão massa-fluxo magnético da nuvem antes do impacto, dada por $N / B=885\left(n_{c, 10} r_{c, 10}\right) / B_{c, 6}$, implicando em $B / B_{c r} \sim 0.7 B_{c, 6} /\left(n_{c, 10} r_{c, 10}\right)$, que é maior do que 1 para $n_{c, 10} \leq 34.8 \mathrm{~cm}^{-3}$ e $n_{c, 10} \leq 69.6$ $\mathrm{cm}^{-3}$ para uma nuvem não chocada com $B=5 \mu \mathrm{G}$ e $B=10 \mu \mathrm{G}$ da Fig. 3.3, respectivamente, antes da interação.

Como na Figura 3.1, os símbolos (isto é, as cruzes e o triângulo) na Fig. 3.2 indicam as condições iniciais consideradas para as interações RSN-nuvens examinadas nas simulações numéricas descritas em Melioli et al. para interações entre RSN e nuvens não magnetizadas. Vemos que quando o capo magnético é incluído, tanto as cruzes quanto o triângulo ficam fora do domínio de formação estelar nos diagramas. Isto significa que para as condições iniciais correspondentes a estes pontos no diagrama a formação estelar é improvável de ocorrer. No artigo de Melioli et al. a aplicação dos resultados do diagrama da Figura 3.1 para uma nuvem não magnetizada com $r_{c}=10 \mathrm{pc}$ e $n_{c} \gtrsim 10 \mathrm{~cm}^{-3}$ à associação estelar jovem de $\beta$-Pictoris (veja a região próxima da cruz no painel inferior esquerdo da Fig. 3.1) permite-nos concluir que esta associação estelar poderia ter se originado de uma interação entre uma nuvem e um RSN com um raio de aproximadamente 52 pc num passado recente. Contudo, a inclusão de um campo magnético efetivo na nuvem com uma intensidade de somente $1 \mu \mathrm{G}$ coloca a cruz correspondente às condições iniciais para a formação desta associação jovem fora da região de formação estelar (veja a cruz no painel inferior esquerdo da Fig. 3.2 $)^{2}$.

\footnotetext{
${ }^{2}$ Devemos lembrar contudo do comentário no parágrafo anterior.
} 


\subsection{Interação RSN-nuvem: simulações numéricas MHD de nuvens magnetizadas e auto-gravitantes}

Como observado anteriormente, existem diversos estudos numéricos do impacto de frentes de choque em nuvens interestelares (e.g., Sgro 1975; McKee \& Cowie 1975; Woodward 1976; Nittmann et al. 1982; Tenorio-Tagle \& Rozyczka 1986; Hartquist et al. 1986; Bedogni \& Woodward 1990; Mac Low et al. 1994; Klein, McKee \& Colella 1994; Anderson et al. 1994; Dai \& Woodward 1995; Xu \& Stone 1995; Jun, Jones \& Norman 1996; Redman, Williams \& Dyson 1998; Jun \& Jones 1999; Lim \& Raga 1999; de Gouveia Dal Pino 1999; Miniati, Jones \& Ryu 1999; Poludnenko, Frank \& Blackman 2002; Fragile et al. 2004; Steffen \& López 2004, Raga, de Gouveia Dal Pino et al. 2002; Fragile et al. 2005; Marcolini et. al 2005; Melioli, de Gouveia Dal Pino \& Raga 2005; Nakamura et al. 2006) a maioria dos quais estão interessados nos efeitos destas interações na destruição da nuvem. Em particular os estudos que incorporam os efeitos do resfriamento radiativo mostram a relevância deste em atrasar a destruição da nuvem e a mistura de seu material no meio interestelar (e.g., Melioli, de Gouveia Dal Pino \& Raga 2005).

Para checar a validade das previsões dos diagramas analíticos construídos para interações envolvendo choques de remanescentes de supernovas e nuvens não magnetizadas, realizamos simulações magneto-hidrodinâmicas 3D com resfriamento radiativo seguindo os passos iniciais destas interações. Também levamos em conta os efeitos da auto-gravidade do gás de modo seguir a evolução do material chocado dentro destas nuvens magnetizadas e checar se este sofre colapso gravitacional ou não, em concordância com nossos diagramas.

Para isto empregamos o código de grade Godunov-MHD desenvolvido originalmente por Kowal \& Lazarian (2007), e testado em Falceta-Gonçalves et al. (2008) e Santos-Lima et al. (2010, 2012), que resolve as equações dinâmicas do gás magnetizado em sua forma conservativa como segue (ver Ap. B):

$$
\frac{\partial \rho}{\partial t}+\nabla \cdot(\rho \mathbf{v})=0
$$




$$
\begin{gathered}
\frac{\partial \rho \mathbf{v}}{\partial t}+\nabla \cdot\left[\rho \mathbf{v} \mathbf{v}+\left(p+\frac{B^{2}}{8 \pi}\right) \mathbf{I}-\frac{1}{4 \pi} \mathbf{B B}\right]=-\rho \nabla \boldsymbol{\Phi} \\
\frac{\partial \mathbf{B}}{\partial t}-\nabla \times(\mathbf{v} \times \mathbf{B})=0
\end{gathered}
$$

$\operatorname{com} \nabla \cdot \mathbf{B}=0$, onde $\rho, \mathbf{v}$ e $p$ são a densidade, velocidade e pressão do plasma, respectivamente, $\mathbf{B}$ é o campo magnético e

$$
\nabla^{2} \boldsymbol{\Phi}=4 \pi G \rho
$$

As equações são resolvidas usando um esquema de Godunov de segunda ordem, com um resolvedor de Riemann HLLD para considerar apropriadamente as velocidades características MHD (Ap. B). Para o termo de auto-gravidade usamos o resolvedor Poisson FACR (Fourier Analysis Cyclic Reduction) em cada passo de tempo. Em Melioli et al. (2006), dada sua importância, os efeitos do resfriamento radiativo atrás dos choques foram considerados explicitamente nas simulações hidrodinâmicas lá apresentadas. No presente trabalho, como o objetivo maior das simulações é estudar o papel do campo magnético e da auto-gravidade na evolução da nuvem, resolvemos as equações sob a suposição de resfriamento radiativo forte.

O grupo de equações acima é completado pela equação de estado $p \propto \rho^{\gamma}$, definindose um índice politrópico $\gamma_{e f f}=1.2$ efetivo para simular o forte resfriamento radiativo. Note que para um gás ideal monoatômico adiabático esse índice é $5 / 3$, enquanto que para um gás ideal isotérmico este índice é 1. Logo, o valor acima caracteriza um gás com resfriamento radiativo quase tão eficiente quanto um gás isotérmico para o qual o resfriamento é instantâneo.

O domínio computacional possui dimensões de $100 \mathrm{pc} \times 100 \mathrm{pc} \times 100 \mathrm{pc}$, correspondendo a uma grade fixa de $256^{3}$ pontos de rede. Um RSN, gerado pela explosão de uma SN com energia $E_{0}=10^{51} \mathrm{erg}$, é inicialmente injetado no canto inferior esquerdo do domínio. Vários testes foram realizados com diferentes densidades iniciais da nuvem $\left(n_{c}\right)$, raios $\left(r_{c}\right)$, e distâncias entre o RSN e a superfície da nuvem $\left(R_{R S N}\right)$. As condições iniciais são descritas na Tabela 3.1. Selecionamos duas destas simulações para mostrar em detalhes a seguir. 
Tabela 3.1: Descrição das simulações

\begin{tabular}{ccccccc}
\hline \hline$n_{c}\left(\mathrm{~cm}^{-3}\right)$ & $r_{c}(\mathrm{pc})$ & $R_{R S N}(p c)$ & codigo & $B(\mu \mathrm{G})$ & Resultado & Predição \\
\hline 10 & 20 & 40 & Hidro & - & colapsa & colapsa \\
10 & 20 & 40 & MHD & 1 & estável & estável \\
50 & 5 & 15 & MHD & 1 & estável & estável \\
10 & 10 & 50 & MHD & 1 & estável & estável \\
100 & 10 & 25 & MHD & 1 & colapsa & colapsa \\
\hline \hline
\end{tabular}

A Fig. 3.4 apresenta os mapas de densidade e vetores do campo magnético no plano central $\mathrm{x}-\mathrm{y}$ que intercepta tanto a nuvem quanto o RSN, para uma nuvem com $n_{c}=10$ $\mathrm{cm}^{-3}, r_{c}=10 \mathrm{pc}, R_{s n r}=50 \mathrm{pc}$ e $B=1 \mu \mathrm{G}$. Estas condições iniciais representam a cruz no painel inferior esquerdo da Fig. 3.2 que encontra-se bem fora da região de formação estelar sombreada no diagrama o qual prevê a destruição da nuvem devido ao impacto. Na simulação da Fig. 3.4, a frente de choque do RSN comprime a nuvem aumentando a densidade de energia magnética. Após a interação (em $t \simeq 1.8 \times 10^{6} \mathrm{~s}$ ), uma cauda de gás é varrida atrás da nuvem pelo RSN em expansão, similarmente aos resultados obtidos, por exemplo, por Murray et al. (1993). A densidade de energia magnética ao longo da cauda também aumenta. Vemos que devido à compressão a nuvem inicialmente torna-se gravitacionalmente instável e começa a colapsar. Em $1.8 \times 10^{6}$ anos, o núcleo está $\sim 10^{3}$ vezes mais denso. A contração da nuvem também causa o aumento da energia magnética que age contra a continuidade do colapso. Posteriormente a nuvem simulada rebota e sofre expansão e evaporação, como visto em $3.3 \times 10^{6}$ anos. Esta reexpansão poderia estar ocorrendo por causa da ineficiência do resfriamento na simulação. Como escolhemos $\gamma_{e f f}=1.2$ e nao incluímos o resfriamento radiativo explicitamente nas equações de evolução do sistema, o gás não está resfriando realisticamente, como esperaríamos em uma nuvem colapsante no meio interestelar. Em tais situações, $\gamma_{\text {eff }}$ poderia ser menor do que a unidade, isto é, o material chocado poderia apresentar temperaturas menores do que aquelas do pré-choque. Por outro lado, Spaans \& Silk (2000) modelaram a química, o balanceamento térmico e a transferência radiativa para diferentes condições do MIS e 
mostraram que uma equação de pressão politrópica pode ser usada como primeira aproximação com índices politrópicos efetivos $\gamma_{e f f} \sim 0.8-1.4$, dependendo das condições locais. No entanto o não colapso verificado na simulação acima poderia ainda ser devido ao aumento na energia magnética.

No artigo de Melioli et al., onde o resfriamento radiativo foi levado em conta apropriadamente, com o cálculo implícito do resfriamento radiativo através da equação de conservação de energia, mas sem considerar o campo magnético ou a auto-gravidade, a simulação hidrodinâmica (Fig. 4 daquele artigo) sugere que a nuvem evapora. Com a introdução do campo magnético (Fig. 3.4) o aumento da pressão magnética nos últimos estágios da evolução da nuvem previne o colapso, como previsto pelo diagrama de formação estelar da Fig. 3.2.

A Figura 3.5 mostra a distribuição de vários parâmetros físicos para o modelo da Fig. 3.4 em $t=1.8 \times 10^{6}$ anos. A densidade de coluna é $N>10^{20} \mathrm{~cm}^{-2}$ no núcleo. Como comentado anteriormente, vemos que no núcleo denso formado durante o colapso inicial há um aumento das pressões do gás e magnética com uma temperatura máxima de $T>10^{4} \mathrm{~K}$, enquanto que a densidade de energia magnética é uma ordem de magnitude maior do que no meio ao redor. O grande aumento da pressão do gás é provavelmente o principal responsável pela reexpansão da nuvem e isto é altamente dependente do resfriamento radiativo. Em uma situação mais realista a escala de tempo do resfriamento $t_{\text {cool }} \sim k T / n \Lambda(T)$ seria mais curta do que a escala de tempo de colapso $t_{g} \sim(G \rho)^{-1}$, o que significa que a temperatura do núcleo seria menor do que $10^{4} \mathrm{~K}$. Todavia, o campo magnético tem também um papel importante em estabilizar a nuvem e suportá-la contra o colapso, como mostrado mais abaixo na Fig. 3.7.

A Figura 3.6 mostra o corte central da distribuição de densidade e vetores do campo magnético para o modelo da Tabela 3.1 para uma nuvem com $n_{c}=100 \mathrm{~cm}^{-3}, r_{c}=10 \mathrm{pc}$ e $B=1 \mu \mathrm{G}$, e um RSN com $R_{R S N}=25$ pc. A evolução neste caso é similar ao modelo anterior. Contudo após $t=1.2 \times 10^{6}$ anos, à medida que a nuvem colapsa a pressão magnética não é capaz de contra-balancear a gravidade e a nuvem continua contraindo. A densidade maior da nuvem neste caso causa um resfriamento radiativo eficiente do material comprimido pelo choque na nuvem o que mantém a energia térmica baixa. Então 
o colapso simplesmente carrega as linhas de campo magnético o que aumenta a densidade de energia magnética, porém este aumento não é suficiente para parar o colapso. Este resultado é consistente com as previsões do diagrama de formação estelar. As condições iniciais do sistema, na Fig. 3.6, correspondem ao símbolo da estrela no painel inferior esquerdo da Fig. 3.2, o qual encontra-se dentro da região sombreada permitida para a formação estelar.

Como comentado anteriormente, a estabilidade de uma nuvem suportada por pressão magnética pode ser quantificada pela razão massa-fluxo magnético, $M / \Phi \simeq N / B$, onde N é a densidade colunar da nuvem (e.g., Crutcher 1999, 2005a, b, c, 2008). Calculamos esta razão massa-fluxo para as nuvens simuladas acima em vários passos de tempo. Os resultados são mostrados na Figura 3.7. Os círculos abertos representam a densidade de coluna máxima para cada instante como uma função do campo magnético médio ao longo de uma dada linha de visada (com máximo $N$ ). Notamos que para o modelo com $n_{c}=10 \mathrm{~cm}^{-3}, r_{c}=10 \mathrm{pc}$ e $R_{R S N}=50 \mathrm{pc}$ (da Fig. 3.4), a nuvem começa a colapsar logo após a interação com o RSN. A densidade de coluna aumenta e atinge o regime instável. A nuvem se contrai e a energia total aumenta. Tanto a densidade de energia interna quanto a magnética inibem um colapso posterior e assim a nuvem reexpande. Apesar de a reexpansão ser provavelmente não totalmente realista, como discutido anteriormente, o estágio final é uma nuvem estável. Para o caso de $n_{c}=100 \mathrm{~cm}^{-3}, r_{c}=10 \mathrm{pc}$, e $R_{R S N}=25$ pc, o mesmo processo ocorre inicialmente. Contudo o aumento das densidades de energia interna e magnética não são suficientes para evitar o contínuo colapso.

Os outros modelos simulados da Tabela 3.1 apresentaram resultados que são também consistentes com os diagramas de formação estelar. O primeiro modelo da tabela é um sistema puramente hidrodinâmico, sem campo magnético, cujas condições iniciais correspondem à cruz no painel inferior direito da Fig. 3.1. Conforme descrito na Tabela 3.1, a simulação numérica da evolução desta interação RSN-nuvem incluindo os efeitos de auto-gravidade leva ao colapso gravitacional da nuvem, o que está de acordo com a previsão do diagrama. Quando o campo magnético de $1 \mu \mathrm{G}$ é incluído num sistema com as mesmas condições iniciais, a simulação numérica MHD mostra que a pressão magnética previne o colapso da nuvem (veja Tabela 3.1). Este modelo é representado pela cruz no 
painel inferior direito da Fig. 3.2, o qual consistentemente, encontra-se fora da região gravitacionalmente instável do diagrama.

O terceiro modelo da Tabela 3.1 também está fora da região instável do diagrama de formação estelar (veja o $X$ no painel superior direito da Fig. 3.2), e a simulação numérica MHD da evolução deste sistema mostra que a nuvem acaba por evaporar devido à forte interação com o choque do RSN.

\subsection{Aplicação a regiões isoladas do MIS}

Podemos aplicar o estudo analítico acima, sintetizado nos diagramas raio do RSN-densidade inicial da nuvem, a regiões isoladas de formação estelar em nossa Galáxia. No artigo de Melioli et al. (2006) focou-se na formação da associação estelar jovem de $\beta$-Pictoris induzida por uma interação entre um RSN e uma nuvem. Aqui, iremos abordar alguns outros exemplos de nosso MIS que apresentam alguma evidência de interações em passado recente com RSNs, como a Grande concha de CO na direção de Escorpião (Reynoso \& Mangum 2001) e a chamada Nuvem Periférica 2 na direção de Cassiopeia (Kobayashi \& Tokunaga 2000; Ruffle et al. 2007, Yasui et al. 2006). Um contra exemplo é a região aparentemente sem formação estelar ao redor do RSN de Vela. De fato, veremos que as condições desta região correspondem a um ponto fora da região sombreada de formação estelar nos diagramas.

A Grande Concha de CO, veja Figura 3.8 é uma estrutura em expansão com uma velocidade de $\sim 3 \mathrm{~km} / \mathrm{s}$, uma massa de $9.3 \times 10^{5} \mathrm{M}_{\odot}$ e uma densidade de $\sim 35 \mathrm{~cm}^{-3}$. Reynoso \& Mangum (2001) sugerem que esta expansão tem origem provavelmente em uma explosão de SN por volta de $\sim 4 \times 10^{6}$ anos atrás. Considerando que a massa da nuvem estava originalmente uniformemente distribuída dentro de uma esfera de raio de 435 " ( $\sim 50 \mathrm{pc}$ ), a densidade inicial da nuvem deveria ser $n_{c} \simeq 30 \mathrm{~cm}^{-3}$. A frente de choque da SN possivelmente induziu a formação de uma estrela tipo O 9.5 que foi detectada como uma fonte IR (IRAS 17146-3723). A Grande Concha de CO tem um raio externo de 50 pc e um raio interno de $\sim 28$ pc (Reynoso \& Mangum, 2001). A idade e a pequena velocidade de expansão sugerem que atualmente tem-se um RSN evoluído e fraco. Se 
considerarmos uma nuvem com a densidade e o raio acima $(\sim 50 \mathrm{pc})$ no momento de uma possível interação com o RSN no regime adiabático podemos localizar este sistema no diagrama de formação estelar dentro da região sombreada como indicado na Figura 3.9, se o RSN possuía um raio entre 2.5 - 72 pc (à época da interação) e uma densidade do meio ambiente $n \simeq 1 \mathrm{~cm}^{-3}$. Contudo, quando incluímos um campo magnético na nuvem de $1 \mu \mathrm{G}$, a faixa possível de raios para o RSN reduz-se a $R_{R S N} \sim 7.8-72$ pc se o raio máximo for calculado usando uma função de resfriamento $\Lambda=3 \times 10^{-27} \mathrm{erg} \mathrm{cm}^{3} \mathrm{~s}^{-1}$.

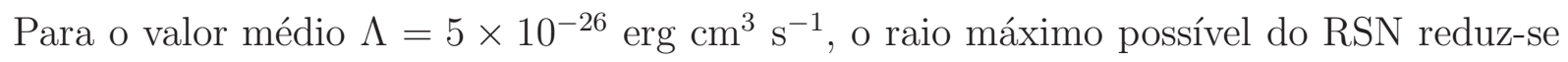
a 38.4 pc. Considerando que o raio atual do RSN evoluído é provavelmente da ordem de 50 pc, a faixa acima de condições iniciais para a interação é bastante plausível.

O remanescente de supernova de Vela possui uma fina concha de HI, quase esférica, expandindo a uma velocidade de $\sim 30 \mathrm{~km} / \mathrm{s}$. Ao invés de estar impactando uma nuvem interestelar, este remanescente está expandindo em um ambiente relativamente denso com evidência de formação de algumas estruturas. Assumindo que Vela está a uma distância de $\sim 300$ pc do Sol, o raio de sua concha é da ordem de 21 pc. A densidade do meio ambiente é $\sim 1$ a $2 \mathrm{~cm}^{-3}$ e a energia inicial da SN é da ordem de de $1-2.5 \times 10^{51} \mathrm{erg}$ (Dubner et al. 1998). Estas condições iniciais correspondem ao quadrado no diagrama da Figura 3.9, o qual encontra-se fora da região sombreada o que é consistente com a ausência de nuvens densas, glóbulos ou estrelas recém nascidas nas vizinhanças deste RSN.

A região de formação estelar nas vizinhanças da Nuvem Periférica 2 é um possível exemplo de uma interação de um RSN na fase radiativa com uma nuvem (veja Figura 3.10). Esta é de fato uma nuvem molecular gigante com um diâmetro de $\sim 30-40$ pc. É um dos complexos de nuvens mais distantes do centro da Via Láctea $(d \simeq 22-28$ kpc; Ruffle et al. 2007) e por esta razão encontra-se em uma região onde a pressão do gás é extremamente pequena e a presença de agentes de formação estelar, como as perturbações devidas aos braços espirais, é improvável. Isto deveria ser um indicativo de que este complexo de nuvens é estável, exceto pela recente detecção de duas associações de estrelas T-Tauri com idades de $\sim 10^{6}$ anos (Kobayashi \& Tokunaga 2000, Yasui et al. 2006). A Nuvem Periférica 2 possui uma temperatura de $20 \mathrm{~K}$, uma densidade de $n_{H_{2}} \sim 10^{4} \mathrm{~cm}^{-3}$, e uma massa estimada de $\sim 10^{4} M_{\odot}$. Existe um RSN velho e grande 
associado a esta nuvem, GSH 138-01-94, que consiste de uma concha de HI com raio de 180 pc expandindo no meio com uma velocidade de $11.8 \pm 0.9 \mathrm{~km} / \mathrm{s}$ e uma idade de aproximadamente $4.3 \times 10^{6}$ anos (Ruffle et al. 2007). De acordo com Ruffle et al. (2007), a formação da estrutura atual e composição química da Nuvem Periférica 2 é possivelmente um resultado da interação deste RSN com o gás interestelar.

Considerando as características do RSN acima (isto é, sua velocidade, raio e uma energia $\sim 10^{51} \mathrm{erg}$ ) e usando a Eq. 2.17, podemos estimar uma densidade para o meio ambiente $n \sim 0.14 \mathrm{~cm}^{-3}$. Também assumindo que a massa da nuvem era originalmente distribuída uniformemente em uma esfera de raio médio $\sim 17.5 \mathrm{pc}$, encontramos que a densidade da nuvem antes da compressão era $n_{c} \sim 14 \mathrm{~cm}^{-3}$. Com estas condições iniciais, a interação RSN-nuvem estaria dentro da região de formação estelar sombreada do diagrama da Figura 3.11 para um RSN com raio entre $\sim 31$ e 102 pc na época da

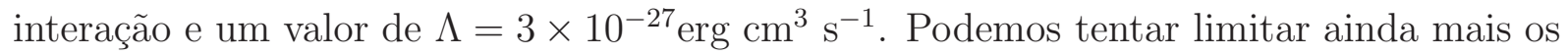
valores possíveis para o raio do RSN assumindo que a interação ocorre em algum momento após o remanescente tornar-se radiativo e antes de $10^{6}$ anos, que é aproximadamente a idade das estrelas observadas. Estes limites implicam em $R_{R S N} \simeq 46 \mathrm{pc}-84 \mathrm{pc}$, como indicado pela região dentro da elipse no diagrama da Figura 3.11.

\subsection{Estimando a eficiência de formação estelar devido a interações RSN-nuvens}

A eficiência de formação estelar (SFE - star formation efficiency) expressa a fração de gás inicialmente presente na nuvem que transformou-se em estrelas. Em nossa galáxia a SFE varia entre 0.3, em regiões de intensa formação estelar, e 0.01 em regiões mais dispersas. Isso demonstra que os mecanismos existentes no MIS que induzem a formação estelar devem possuir uma eficiência baixa na Galáxia atualmente, frente aos mecanismos de suporte interno nas nuvens contra o colapso, como os campos magnéticos, e também graças à inexistência de ambientes muito densos e confinados com altas concentrações de gás e poeira, como é o caso de galáxias com alta taxa de formação estelar, como nas 
galáxias de 'starburst'.

Da análise realizada aqui podemos tentar estimar a eficiência de formação estelar que a interação entre RSNs e nuvens difusas produzem e comparar com os valores observados de forma a ver a contribuição deste mecanismo na SFE total da Galáxia. Os diagramas construídos neste trabalho fornecem um domínio para a ocorrência de formação estelar através destas interações, em outras palavras, estabelecem as condições em que tais interações isoladas podem levar a um colapso gravitacional bem sucedido do material comprimido da nuvem. Para calcular a SFE global destas interações temos que calcular primeiro sua probabilidade de ocorrência na Galáxia. Considerando que uma vez formado um RSN irá propagar e comprimir o meio difuso ao redor proporcionando o tipo de interações que estamos examinando, a probabilidade de estas interações ocorrerem deve ser proporcional a:

$$
\begin{gathered}
f_{R S N} \simeq N_{S N I I} \Delta t_{R S N}\left(R_{R S N}\right) \frac{A_{R S N}\left(R_{R S N}\right)}{A_{G}} \\
\simeq 10^{-1} \frac{N_{S N I I}}{1.7 \times 10^{-2} \text { anos }} \frac{\Delta t_{R S N}}{5 \times 10^{5} \text { anos }} \frac{A_{R S N}}{2.5 \times 10^{3} p c^{2}}\left(\frac{A_{G}}{4 \times 10^{8} p c^{2}}\right)^{-1}
\end{gathered}
$$

onde assumimos um disco galático fino e homogêneo com um raio de 20 kpc para calcular a área galática $\left(A_{G}\right)$, e onde $N_{S N I I}$ é a taxa de explosões de SNII (e.g. Cappellaro, Evans \& Turatto 1999), $\Delta t_{R S N}\left(R_{R S N}\right)$ é o tempo de vida e $A_{R S N}$ é a área de um RSN e ambos dependem de $R_{R S N}$. Desde que nem todo o volume galático é preenchido com nuvens, a quantidade acima deve ser multiplicada pelo fator de preenchimento das nuvens difusas neutras de modo a obtermos uma probabilidade aproximada da ocorrência de interações entre RSN e nuvens. Se considerarmos a quantidade de gás que está concentrada dentro dos complexos de nuvens na fase fria do meio interestelar, o fator de preenchimento do volume correspondente é $f_{c} \simeq 5 \%$ (e.g., de Avillez \& Breitschwerdt 2005), e então a probabilidade de ocorrência destas interações será dada por $f_{R S N-c} \simeq f_{R S N} \times f_{c}$. Podemos escrever o raio do remanescente em função do tempo para o caso de expansão adiabática 


$$
R_{R S N, a}(t)=\left(\frac{25 E_{0} t^{2}}{4 \pi \rho}\right)^{1 / 5} \sim 13\left(\frac{E_{51}}{n}\right)^{1 / 5} t_{4}^{2 / 5} p c,
$$

e para o caso de expansão radiativa

$$
R_{R S N, r}(t) \sim 19\left(\frac{E_{51}^{0.23}}{n^{0.26}}\right) t_{4}^{2 / 7} \mathrm{pc} .
$$

e substituir as Eq. 3.7 e 3.8 para $\Delta t_{R S N}\left(R_{R S N}\right)$ na Eq. (3.6) encontramos:

$$
f_{R S N-c}\left(R_{R S N}, a\right)=f_{R S N} \times f_{c} \simeq 9.5 \times 10^{-3} R_{R S N, 50}^{9 / 2}
$$

para uma interação com um RSN no regime adiabático e

$$
f_{R S N-c}\left(R_{R S N}, r\right)=2.9 \times 10^{-3} R_{R S N, 50}^{11 / 2}
$$

para uma interação com um RSN no regime radiativo.

Esta probabilidade pode ser multiplicada pela fração de massa do gás chocado que é gravitacionalmente instável dentro do domínio de formação estelar de nossos diagramas de interação RSN-nuvem de modo a obter uma eficiência de formação estelar global efetiva para estas interações. Usando a massa de Jeans calculada para o gás chocado (Eqs. 2.36, 2.37 e 2.41) como um limite inferior aproximado para a fração da nuvem que deveria colapsar para formar estrelas, obtemos:

$$
s f e_{R S N-c} \simeq f_{R S N-c} \frac{m_{J}}{m_{c}}
$$

o qual no caso de uma nuvem não magnetizada interagindo com um RSN na fase adiabática dá (de acordo com Eq. 2.36):

$$
s f e_{R S N-c, a} \simeq 5.4 \times 10^{-3} \frac{T_{c, 100}^{2} R_{R S N, 50}^{6}}{n_{c, 10} r_{c, 10}^{3} E_{51}^{1 / 2} I_{5}}
$$

e para o caso da interação de uma nuvem não magnetizada com um RSN na fase radiativa (usando Eq. 2.37):

$$
s f e_{R S N-c, r} \simeq 1.5 \times 10^{-3} \frac{T_{c, 100}^{2} R_{R S N, 50}^{6} n^{0.41}}{n_{c, 10} r_{c, 10}^{3} I_{5} E_{51}^{0.8} f_{10}^{1 / 2}}
$$


Para interações envolvendo nuvens magnetizadas temos:

$$
s f e_{R S N-c, B} \simeq \frac{5 f_{R S N-c}\left(R_{R S N}\right)}{y^{1 / 2} n_{c, 10}^{3 / 2} r_{c, 10}^{3}}\left[4.14 T_{c, 100}+\frac{4 y B_{6}^{2}}{n_{c, 10}}\right]^{3 / 2}
$$

onde $y$ deve ser substituído pela Eq. 2.29. Usando as Eqs. 2.30 e 2.22 temos a SFE para interações com RSNs adiabáticos e usando as Eqs. 2.31 e 2.23 temos a SFE para interações com RSNs radiativos.

Como exemplo, as Figuras 3.12 e 3.13 mostram gráficos da SFE aproximada calculada para interações RSN-nuvens como uma função do raio do RSN para diferentes valores do campo magnético da nuvem e para diferentes valores da densidade da nuvem. Desde que a SFE calculada é dependente do vínculo da massa de Jeans (veja as Eqs. 3.9 e 3.10), a região que define o domínio permitido para a formação estelar nestes novos gráficos é limitada pelas outras duas condições em $R_{R S N}$, isto é, as condições de penetração do choque na nuvem e de não destruição da nuvem calculadas na Secção 2.3. O aumento do campo magnético tende a deslocar a região de SF para valores menores do raio do RSN tanto para um RSN no regime adiabático quanto no regime radiativo ${ }^{3}$. A linha vertical nos diagramas estabelece o raio de transição do RSN do regime adiabático (esquerda) para o radiativo (direita) para as condições iniciais específicas dos diagramas. Em outras palavras, o domínio relevante nos diagramas da Figura 3.12 é do lado esquerdo desta linha, enquanto que na Fig. 3.13, está do lado direito.

Notamos que a SFE efetiva encontrada para estas interações é geralmente menor do que os valores típicos observados para a Galáxia e o intervalo de valores possíveis para a SFE diminui com o aumento do campo magnético na nuvem. Vemos também que o espaço de parâmetros que permite valores de SFE próximos dos observados (0.01-0.3) é muito reduzido. Os diagramas da Fig. 3.13, aplicados a interações envolvendo RSN mais evoluídos, na fase radiativa, mostram resultados interessantes. Nesta fase o RSN é menos

\footnotetext{
${ }^{3}$ Como visto na Secção 3.1, este desvio, que é devido ao vínculo da massa de Jeans quando consideramos um campo magnético não nulo normal à frente de choque e deve afetar somente a compressão inicial e o colapso da nuvem chocada. Posteriormente, o colapso ocorrerá principalmente na direção paralela à $\vec{B}$. Assim, nas Figs. 3.12 e 3.13, o diagrama superior deveria ser considerado o mais apropriado quando comparado com as observações.
} 
poderoso e portanto, menos destrutivo do que na fase adiabática. Além disso, é muito mais expandido aumentando a possibilidade de colisão com nuvens. Isto explica os maiores valores atingidos no cálculo da SFE $s f e_{R S N-c}$. Contudo o espaço de parâmetros que permite interações bem sucedidas para a formação estelar do lado direito dos diagramas é mais fina do que nas interações com RSNs adiabáticos (Fig. 3.12) e desaparece quando $B_{c}=10 \mu \mathrm{G}$.

Os resultados acima sugerem que estas interações não são suficientes para explicar a SFE observada na Galáxia na presença ou na ausência de campo magnético na nuvem. Eles são consistentes com a análise anterior realizada por Joung \& Mac Low (2006) onde os autores concluíram que a turbulência induzida por Supernovas tende a inibir a formação estelar global ao invés de induzi-la. Devemos notar contudo que eles basearam suas conclusões no cálculo da taxa de formação estelar (SFR - star formation rate), ao invés de SFE, através de simulações do MIS considerando a injeção de turbulência devido às SNs e seu cálculo da SFR foi realizado usando um valor fixo da SFE tomado das observações $($ sfe $\sim 0.3)$.

\subsection{Conclusões}

Apresentamos aqui um estudo de interações isoladas entre RSNs e nuvens difusas neutras com o objetivo de determinar as condições nas quais estas geram o colapso gravitacional do material chocado e a formação estelar, ao invés da destruição da nuvem. Um estudo preliminar destas interações desprezando os efeitos do campo magnético na nuvem foi realizado anteriormente por Melioli et al. (2006). Neste trabalho, incorporamos estes efeitos e derivamos um conjunto de condições para estas interações. A primeira condição determina o limite da massa de Jeans para o material comprimido da nuvem no impacto. Um segundo estabelece a extensão da penetração da frente de choque do RSN dentro da nuvem antes de esmorecer devido a perdas radiativas. O choque deve ter energia suficiente para comprimir tanto material da nuvem quanto possível antes de enfraquecer. Um terceiro vínculo estabelece a condição para que esta frente de choque não seja forte demais e destrua a nuvem completamente. Construímos então diagramas do raio do remanescente de 
supernova versus a densidade inicial da nuvem onde este grupo de vínculos delimitam um domínio dentro do qual estas interações RSN-nuvem podem resultar em formação estelar (Secção 2.3). Como esperado, encontramos que um campo magnético embebido na nuvem normal à frente de choque com uma intensidade de $1 \mu \mathrm{G}$ diminui levemente o domínio de formação estelar no diagrama quando comparado com o caso não magnetizado. O campo magnético possui um papel dominante sobre o vínculo da massa de Jeans causando um deslocamento da região de formação estelar permitida para densidades da nuvem maiores no diagrama. Quando maiores intensidades de campos magnéticos são considerados (5-10 $\mu \mathrm{G})$, o encolhimento da região permitida é muito mais significativo. Devemos enfatizar contudo que, embora as observações indiquem valores típicos de $B_{c} \simeq 5-10 \mu \mathrm{G}$ para estas nuvens neutras, ao considerarmos campos uniformes e normais à velocidade de impacto no cálculo dos vínculos do diagrama, maximizamos os efeitos do campo contra o colapso gravitacional. Deveríamos então considerar como um resultado mais realista o obtido quando $B_{c} \simeq 1 \mu \mathrm{G}$ é empregado. Estes diagramas derivados de considerações analíticas simples fornecem uma ferramenta útil para identificar regiões de onde a formação estelar pode ser induzida pelo impacto de uma onda de choque de supernova.

Para testar esses diagramas construídos analiticamente, também realizamos simulações numéricas 3D MHD do impacto entre um RSN e uma nuvem na presença de autogravidade e campos magnéticos para diferentes condições iniciais (Secção 3.2) traçando a evolução destas interações e identificando as condições que levam ou ao colapso da nuvem e a formação estelar ou à completa destruição e mistura com o meio ambiente. O resfriamento radiativo nas simulações numéricas, o qual é determinante para o colapso do material chocado, foi considerado através da adoção de uma equação de estado politrópica com um expoente efetivo $\gamma_{e f f} \sim 2$. Encontramos que os resultados numéricos são consistentes com os estabelecidos pelos diagramas do raio do RSN versus densidade da nuvem.

Aplicamos os resultados a algumas regiões no MIS com evidência de interações como as examinadas neste trabalho. No artigo de Melioli et al. (2006) a aplicação dos resultados dos diagramas de formação estelar para uma nuvem não magnetizada (como na Figura 3.2) à associação estelar jovem de $\beta$-Pictoris no MIS local levou à conclusão de que esta 
associação estelar poderia ter se originado de uma interação de um RSN com uma nuvem de raio $\sim 10$ pc e densidade $\sim 20 \mathrm{~cm}^{-3}$ e um RSN com raio $\sim 42$ pc. Contudo no trabalho atual encontramos que com a inclusão de um campo magnético efetivo na nuvem com uma intensidade de apenas $1 \mu \mathrm{G}$ esta interação é improvável de produzir tal associação estelar (Figura 3.2, cruz no painel inferior esquerdo), ao menos não para as condições iniciais previstas na literatura para aquele sistema (veja também Melioli et al. 2006). No caso do sistema em expansão Grande Concha de CO-estrela O9.5, encontramos que a formação estelar local poderia ter sido induzida nesta região se, à época da interação, o RSN que originou esta concha em expansão estivesse na fase adiabática e tivesse um raio entre $\sim 8$ pc -29 pc e atingisse uma nuvem magnetizada com densidade por volta de $30 \mathrm{~cm}^{-3}$ e $B \sim 1 \mu \mathrm{G}$ (Figura 3.9). Um outro exemplo é a região de formação estelar próxima à Nuvem Periférica 2. Esta é um dos complexos de nuvens mais distantes do centro galático onde perturbações externas deveriam ser raras. Porém, foram recentemente detectadas duas associações jovens de estrelas T-Tauri nesta região, que poderiam ter sido formadas da interação de um RSN na fase radiativa com a nuvem se a interação iniciou-se a $\lesssim 10^{6}$ anos, e se o RSN tivesse um raio de $R_{R S N} \simeq 46$ pc -84 pc e a nuvem magnetizada, uma densidade de aproximadamente $n_{c} \sim 14 \mathrm{~cm}^{-3}$ (Figura 3.11).

Finalmente, embora este estudo tenha se concentrado em interações isoladas envolvendo RSNs e nuvens, usamos os resultados dos diagramas para estimar a contribuição destas interações na formação estelar global. A eficiência de formação estelar efetiva encontrada para este tipo de interação é geralmente menor do que os valores observados para nossa Galáxia (sfe 0.01-0.3, Figuras 3.12 e 3.13). Este resultado parece ser consistente com a análise anterior de Joung \& Mac Low (2006) e sugere que estas interações são poderosas o suficiente para induzir a formação de estruturas, turbulência supersônica (veja por exemplo a simulação da Figura 3.4) e eventualmente formação estelar local, mas não parecem ser suficientes para induzir a formação estelar global em nossa galáxia ou em outras galáxias com formação estelar normal, nem mesmo quando o campo magnético é desprezado. Concluindo, o pequeno tamanho do domínio permitido para a formação estelar nos diagramas e os resultados para a SFE estimada indicam que estas interações devem levar mais frequentemente à fragmentação e destruição das nuvens do que ao seu 
colapso gravitacional. 

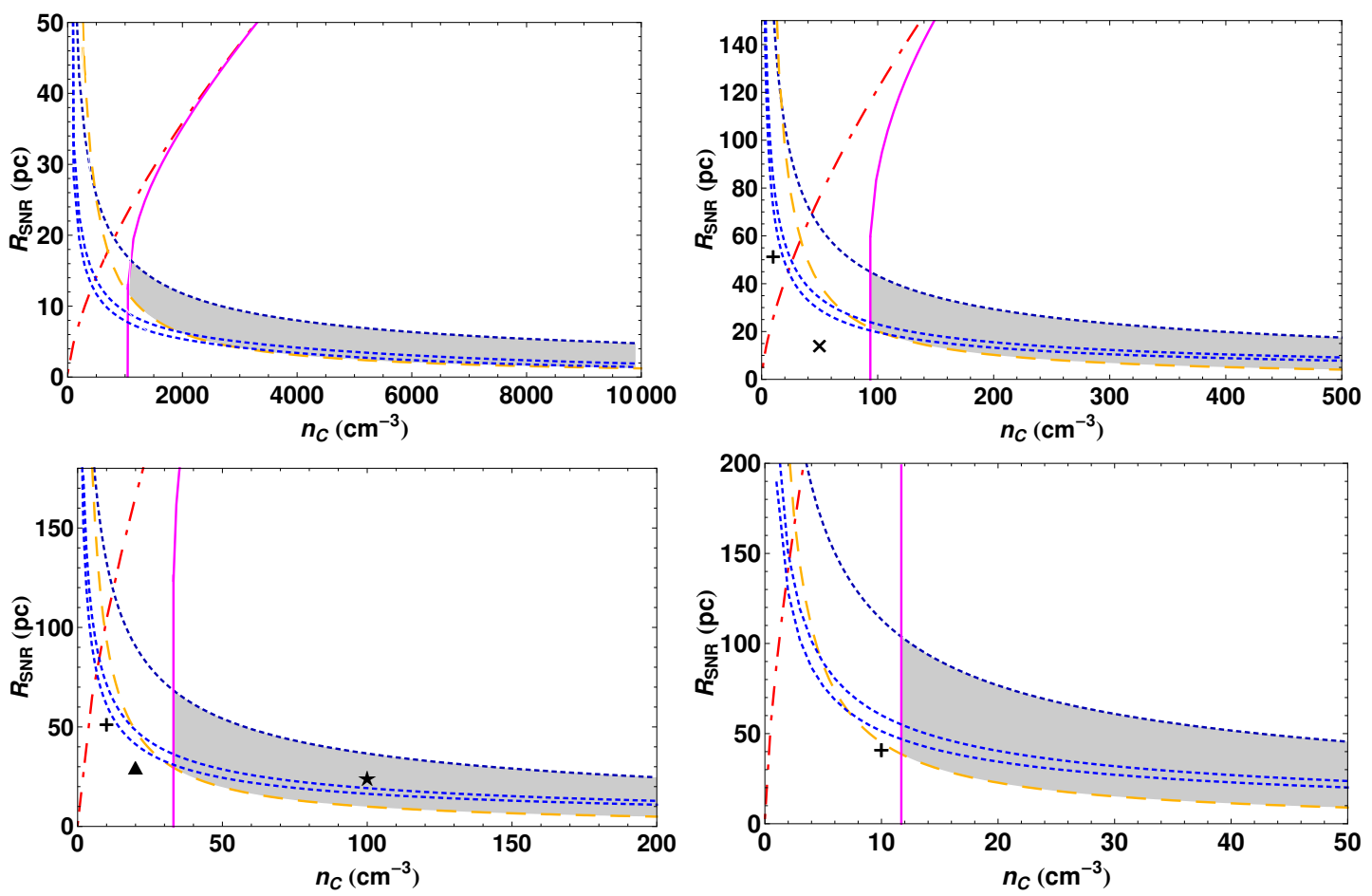

Figura 3.2: Vínculos para o raio do RSN versus a densidade da nuvem para 4 diferentes raios de nuvens como na Figura 3.1, mas agora para nuvens magnetizadas com um campo magnético de $\mathrm{B}=1 \mu \mathrm{G}$ normal à frente de choque. Painéis superiores: à esquerda, nuvem com $r_{c}=1$ pc; à direita, $r_{c}=5$ pc. Painéis inferiores: à esquerda, nuvem com $r_{c}=10 \mathrm{pc}$; à direita, $r_{c}=20$ pc. Linha tracejada (amarela): limite superior para a completa destruição da nuvem após um encontro com um RSN adiabático derivado da Eq. (2.46); linha sólida (magenta): limite máximo superior para a nuvem chocada atingir a massa de Jeans derivado da Eq. (2.38); linha pontilhada (azul): limite superior para a frente de choque viajar dentro da nuvem antes de ser desacelerada a velocidades subsônicas derivado da Eq. (2.51) para diferentes valores da função de resfriamento $\Lambda\left(T_{s h}\left(t_{s t}\right)\right)=10^{-25} \mathrm{erg} \mathrm{cm}^{3} \mathrm{~s}^{-1}$ (curva inferior), $5 \times 10^{-26} \mathrm{erg} \mathrm{cm}^{3} \mathrm{~s}^{-1}$ (curva central), e $3 \times 10^{-27} \mathrm{erg} \mathrm{cm}^{3} \mathrm{~s}^{-1}$ (curva superior). A linha vermelha ponto-tracejada corresponde ao vínculo da massa de Jeans sem a presença de campo magnético (veja o texto para detalhes). A área sombreada define a região onde a formação estelar pode ser induzida pela interação RSN-nuvem (entre as linhas sólida, tracejada e pontilhada). As cruzes e o triângulo nas imagens indicam as condições iniciais assumidas para as nuvens nas simulações numéricas descritas na Secção 4.2 de Melioli et al. e corresponde às condições iniciais das simulações MHD das Figuras 3.4 e 3.5. A estrela corresponde às condições iniciais para a simulação MHD da Figura 3.6. 

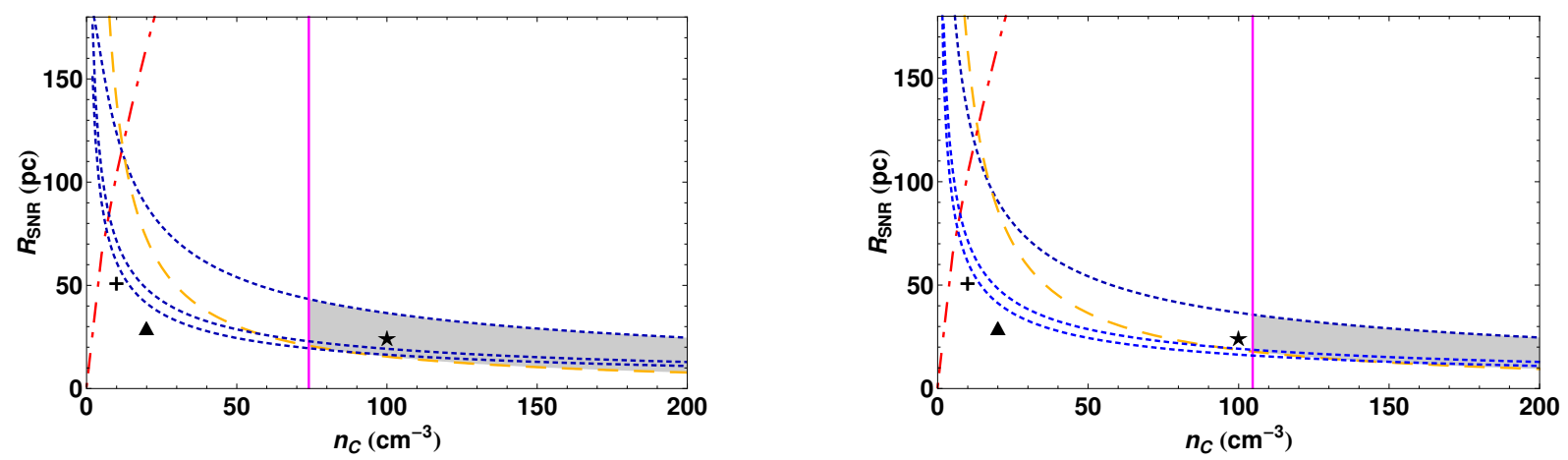

Figura 3.3: $\quad$ O mesmo que na Figura 3.2 para uma nuvem com $r_{c}=10$ pc mas agora considerando $B=5 \mu \mathrm{G}$ (painel à esquerda) e $B=10 \mu \mathrm{G}$ (painel à direita). 

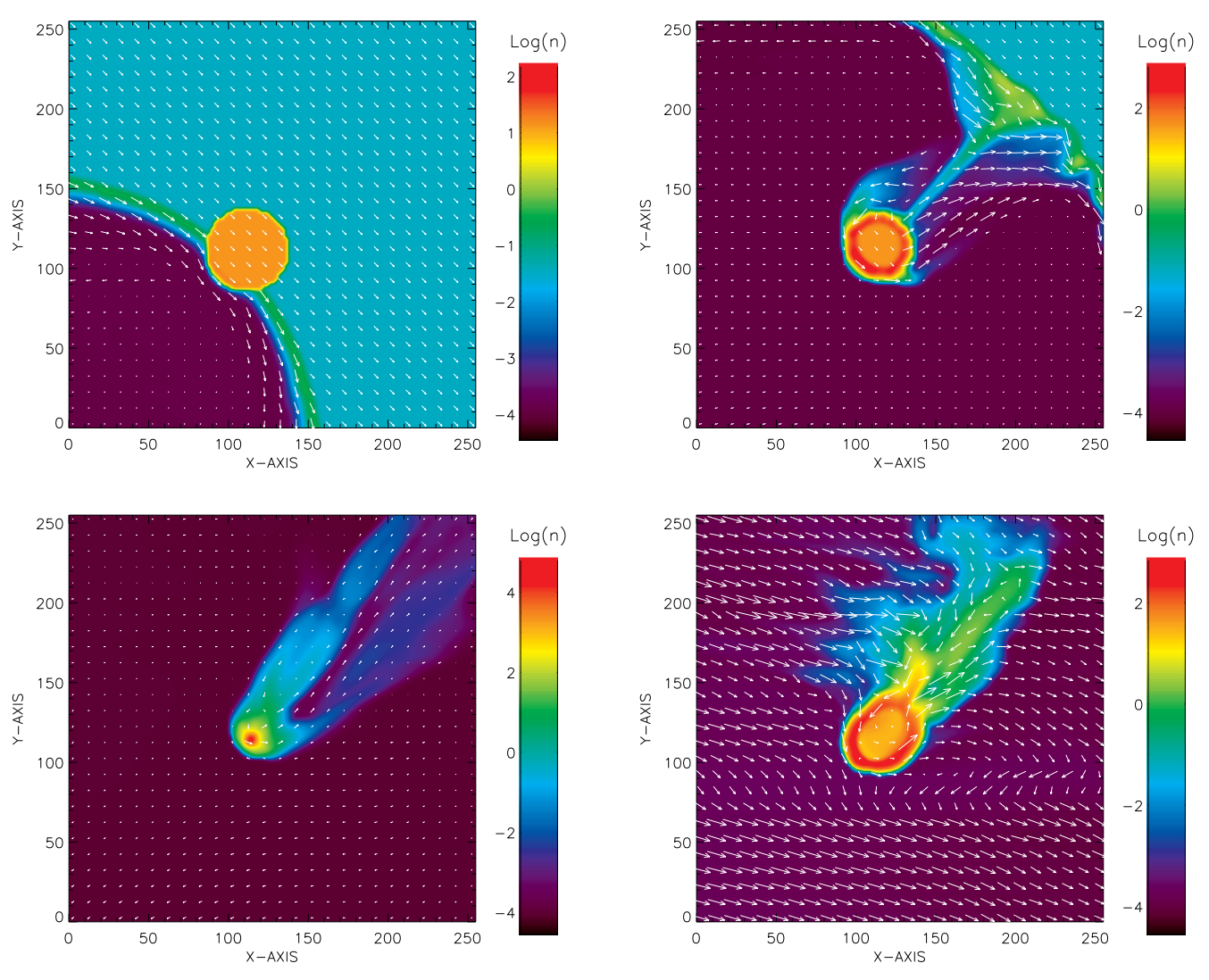

Figura 3.4: Mapas em escala de cor do corte central da evolução da distribuição de densidade e vetores de campo magnético para o modelo $n_{c}=10 \mathrm{~cm}^{-3}, r_{c}=10 \mathrm{pc}$, $R_{R S N}=50 \mathrm{pc}$, e $B=1 \mu \mathrm{G}$. Os tempos evolutivos são $t=3 \times 10^{5}$ anos (painel superior à esquerda); $8 \times 10^{5}$ anos (painel superior à direita); $1.8 \times 10^{6}$ anos (painel inferior à esquerda); e $3.3 \times 10^{6}$ anos (painel inferior à direita). O MIS onde o RSN expande tem densidade numérica $n=0.05 \mathrm{~cm}^{-3}$ e uma temperatura $10^{4} \mathrm{~K}$. Os vetores do campo são normalizados pelo máximo valor atingido em cada instante apresentado. 

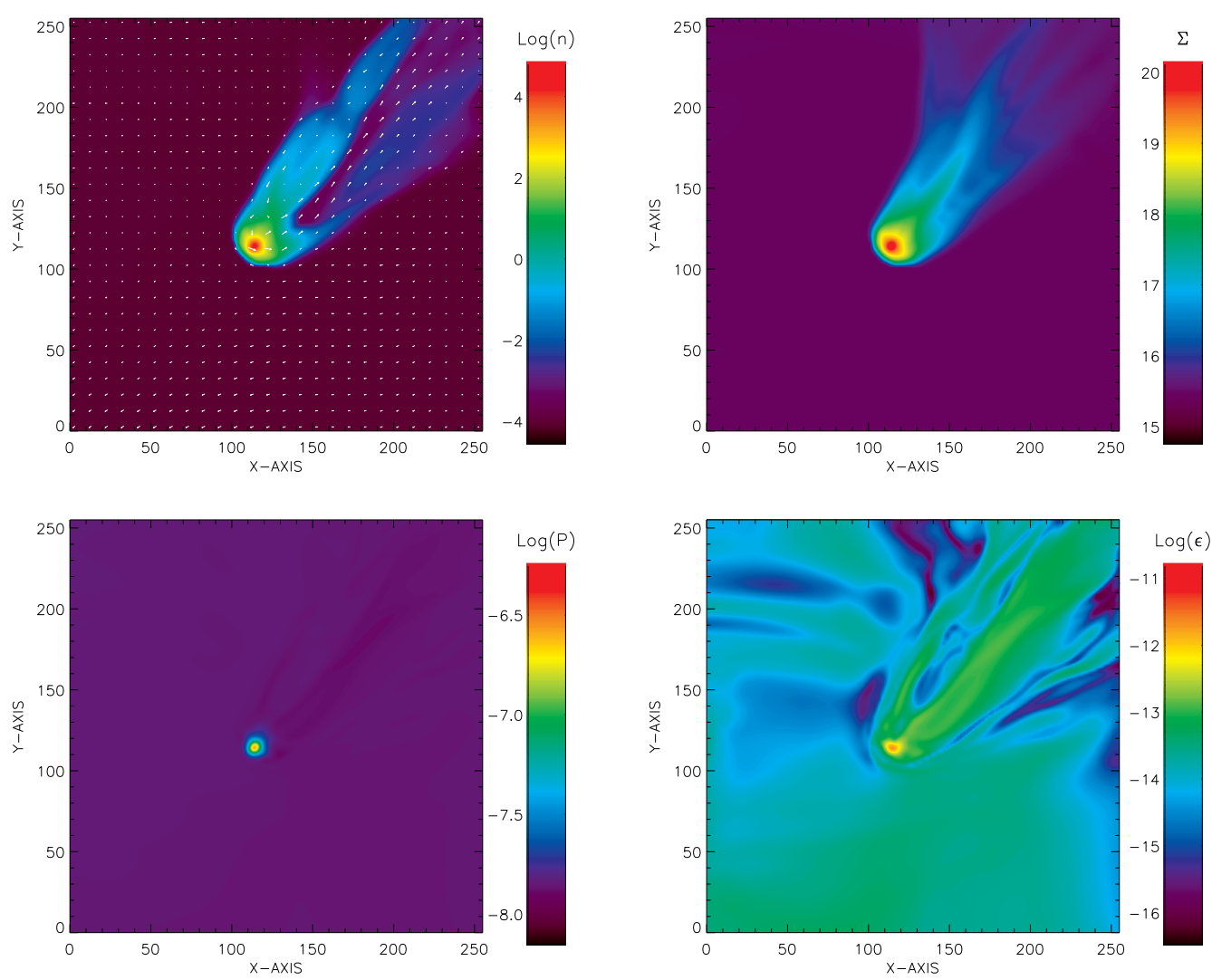

Figura 3.5: Mapas em escala de cor do plano central da densidade com vetores de campo magnético, densidade de coluna, pressão do gás e densidade de energia magnética para o mesmo modelo da Figura $3.4 \mathrm{em} t=1.8 \times 10^{6}$ anos. 

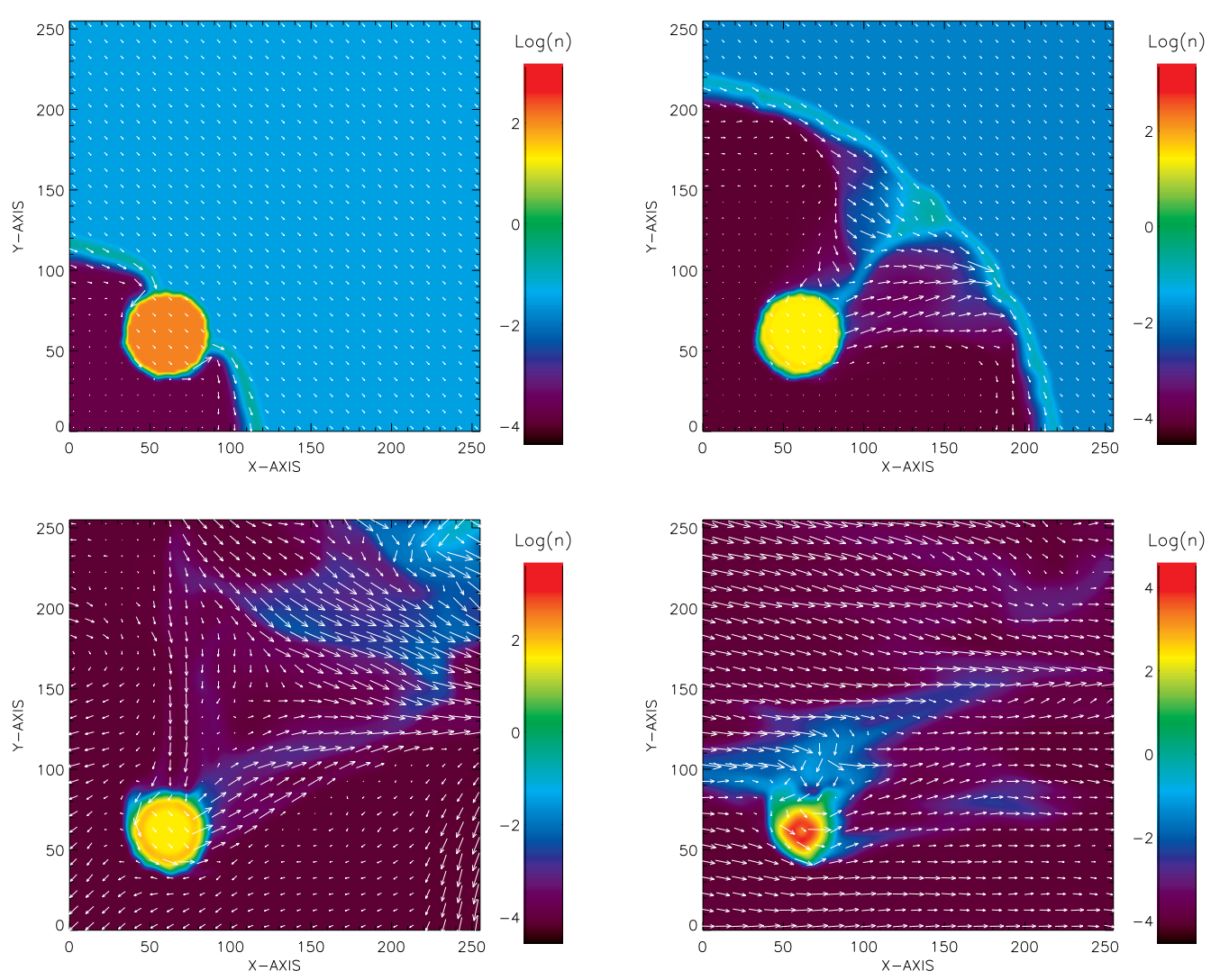

Figura 3.6: Mapas da escala de cor do plano central da evolução da distribuição de densidade e vetores do campo magnético para o modelo com $n_{c}=100 \mathrm{~cm}^{-3}, r_{c}=10 \mathrm{pc}$, $R_{R S N}=25 \mathrm{pc}$, e $B=1 \mu \mathrm{G}$. Os tempos evolutivos são $t=2 \times 10^{5}$ anos (imagem superior à esquerda); $5 \times 10^{5}$ anos (imagem superior à direita); $1.2 \times 10^{6}$ anos (imagem inferior à esquerda); e $2.5 \times 10^{6}$ anos (imagem inferior à direita). Os vetores são normalizados pelo máximo em cada imagem. 

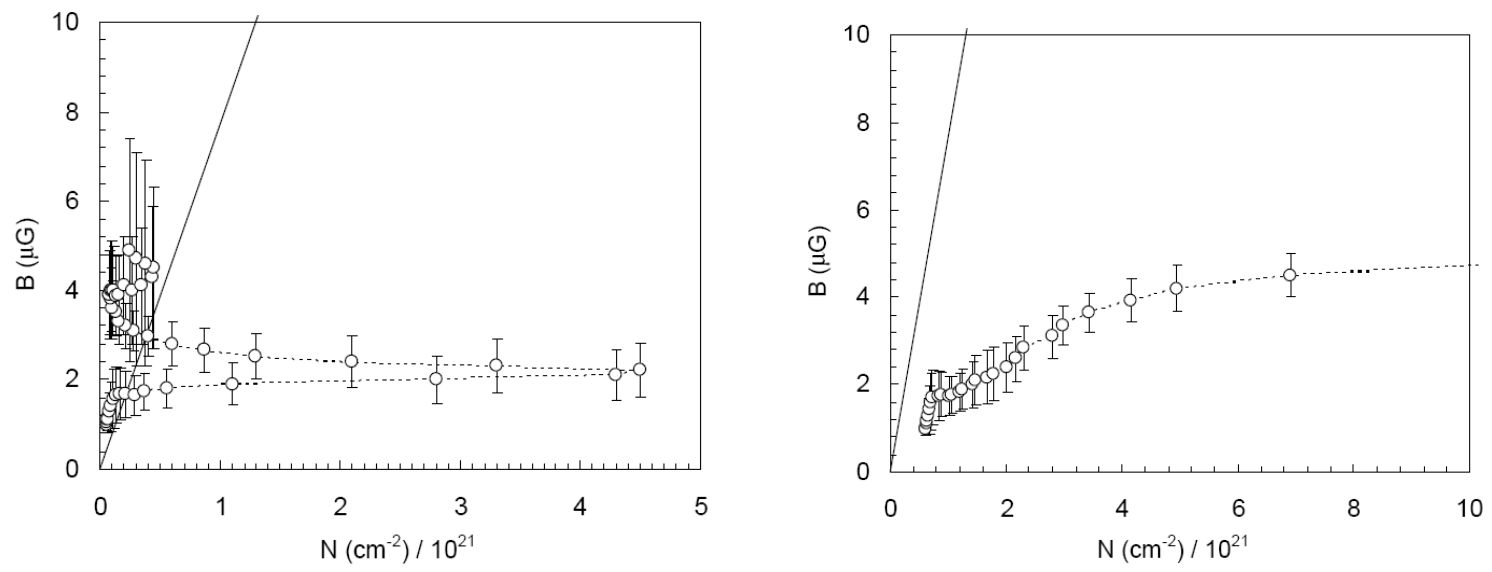

Figura 3.7: Relação massa-fluxo obtida para vários passos temporais das simulações descritas no texto, para as condições da nuvem: $n=10 \mathrm{~cm}^{-3}, r_{c}=10 \mathrm{pc}, R_{S N}=50$ pc (esquerda) (quarto modelo na Tabela 3.1), e $n=100 \mathrm{~cm}^{-3}, r_{c}=10 \mathrm{pc}, R_{S N}=25$ pc (direita) 'quinto modelo na Tabela 3.1). A linha sólida em cada diagrama dá a razão massa fluxo crítica a qual separa o domínio gravitacionalmente instável (do lado direito da linha) do estável (lado esquerdo da linha). 


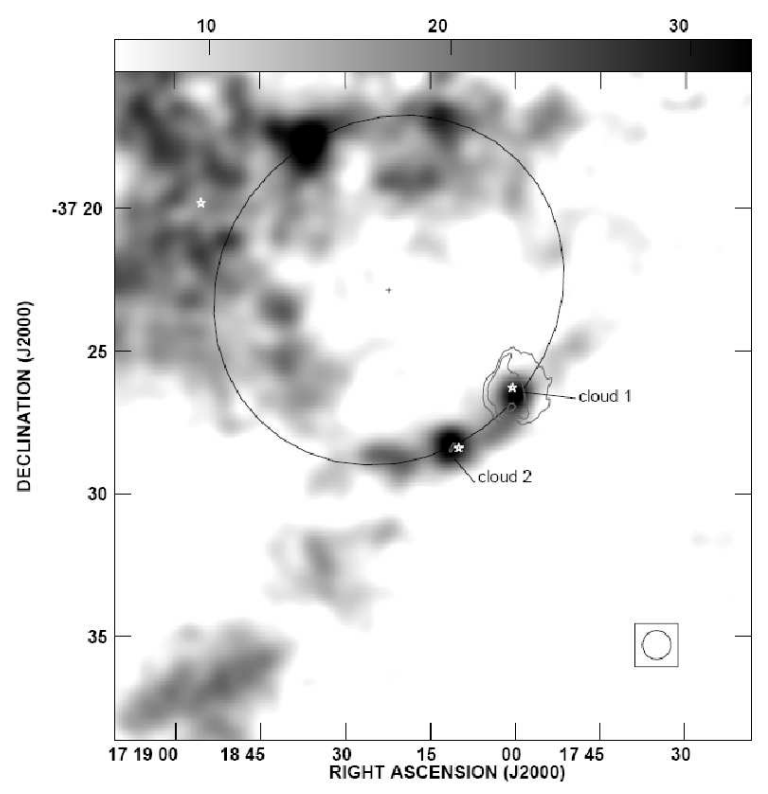

Figura 3.8: Região do RSN G349.7+0.2. A imagem mostra a emissão do CO integrado entre +4.5 e $+20.5 \mathrm{~km} / \mathrm{s}$ de toda estrutura de CO englobando a nuvem e o RSN. A escala cinzenta está em K.km/s e é mostrada no alto da imagem. A imagem original foi formada de imagens com intervalos de 60" × 60" (traçados no canto direito inferior). Uma elipse é ajustada aos picos do CO. As estrelas brancas indicam a posição de três fontes IRAS pontuais. Alguns contornos representativos da emissão do continuo de G349.7+0.2 são incluídos como linhas contínuas cinzentas. (Extraído de Reynoso \& Mangum (2001) Reynoso \& Mangum (2001)). 


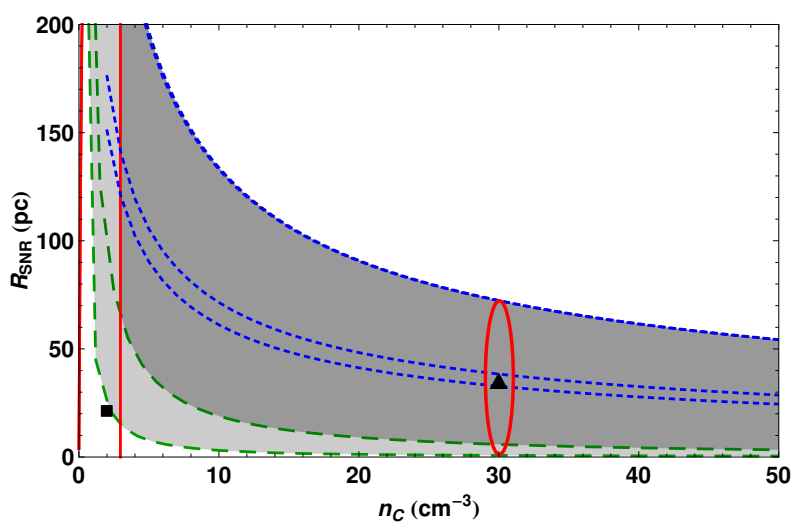

Figura 3.9: Diagrama apresentando o domínio no espaço de parâmetros para SF para uma interação de uma nuvem com um RSN no regime adiabático. A região sombreada cinza-claro corresponde à região permitida para formação estelar para interações sem campo magnético enquanto que a cinza-escuro corresponde à interação com uma nuvem magnetizada com $B=1 \mu \mathrm{G}$. Como nas figuras anteriores, as linhas pontilhadas azuis de cima para baixo representam o vínculo para a penetração do choque para três valores diferentes de $\Lambda=3 \times 10^{-27} \mathrm{erg} \mathrm{cm}^{3} \mathrm{~s}^{-1} ; 5 \times 10^{-27} \mathrm{erg} \mathrm{cm}^{3} \mathrm{~s}^{-1}$; e $1 \times 10^{-25} \mathrm{erg} \mathrm{cm}^{3} \mathrm{~s}^{-1}$, respectivamente. As outras condições iniciais são $r_{c}=50 \mathrm{pc}$ e $T_{c}=100 \mathrm{~K}$. A densidade do meio ambiente é $n=1 \mathrm{~cm}^{-3}$. O triângulo representa as condições inciais para a interação envolvendo a Grande Concha de CO, centrado em $n_{c}=30 \mathrm{~cm}^{-3}$ e $R_{R S N}=36$ pc, enquanto a elipse vermelha indica uma faixa de valores possíveis para o raio do RSN interagente. O quadrado representa as condições iniciais para o sistema de Vela (veja o texto para detalhes). 


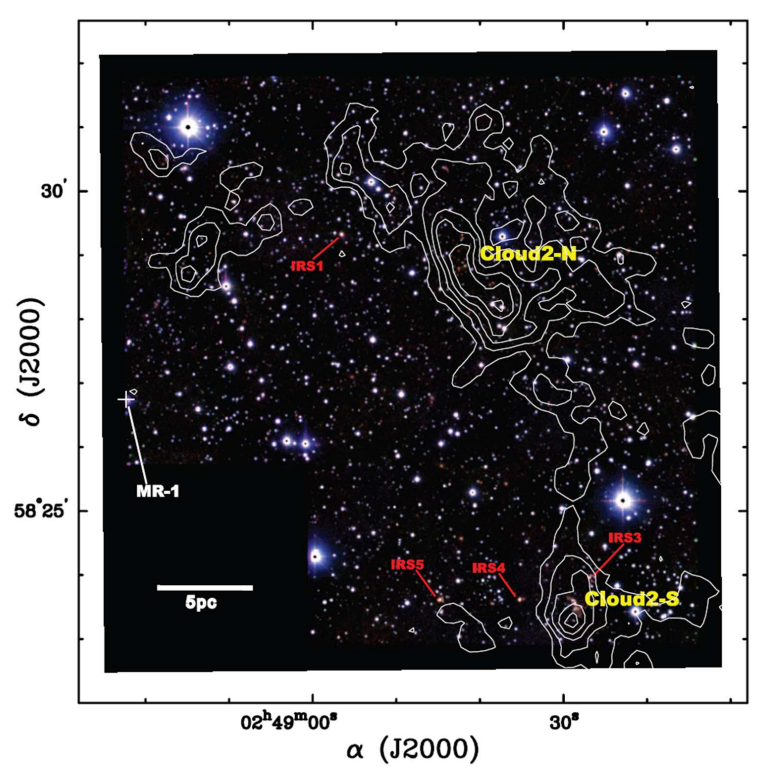

Figura 3.10: Imagem em três cores, JHK, no infravermelho próximo da Nuvem Periférica 2 obtida com a câmera QUIRC NIR. Os dados em ${ }^{12} \mathrm{CO}(1-0)$ estão sobrepostos (contornos brancos) (de Kobayashi et al. 2008). 


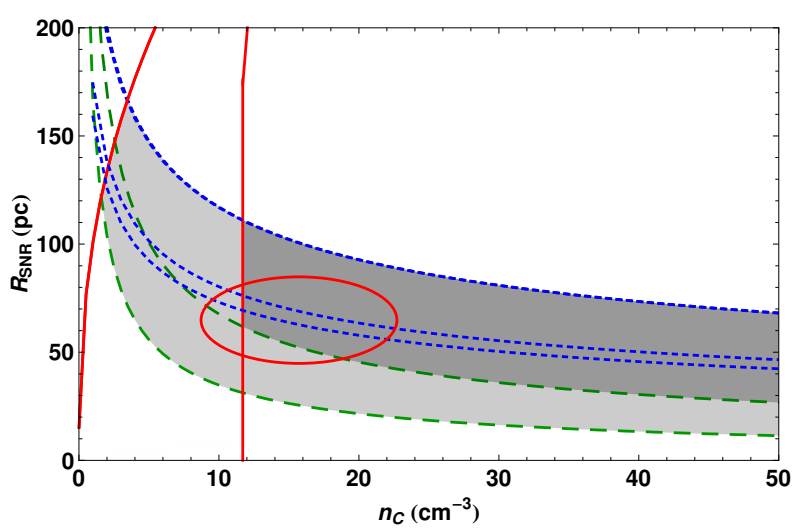

Figura 3.11: Diagrama apresentando o domínio para a formação estelar no espaço de parâmetros para uma ima interação entre uma nuvem e um RSN no regime radiativo expandindo em um meio ambiente com $n \sim 0.14 \mathrm{~cm}^{-3}$. A região sombreada cinzaclaro corresponde à região permitida para a formação de estrelas para interações com nuvens sem a presença de campo magnético enquanto que a cinza-escuro corresponde à região permitida para a SF para interações com uma nuvem com um campo magnético de $B=1 \mu \mathrm{G}$. Como nas figuras anteriores, as linhas pontilhadas azuis de cima para baixo representam o vínculo para a penetração do choque para três valores diferentes de $\Lambda=3 \times 10^{-27} \mathrm{erg} \mathrm{cm}^{3} \mathrm{~s}^{-1} ; 5 \times 10^{-27} \mathrm{erg} \mathrm{cm}^{3} \mathrm{~s}^{-1} ;$ e $1 \times 10^{-25} \mathrm{erg} \mathrm{cm}^{3} \mathrm{~s}^{-1}$, respectivamente. As outras condições iniciais são $r_{c}=20$ pc e $T_{c}=100 \mathrm{~K}$, as quais são apropriadas para o sistema Nuvem Periférica 2-RSN GSH 138-01-94. A elipse indica uma possível faixa no espaço de parâmetros para que a interação entre a Nuvem Periférica 2 e o RSN resulte em um colapso gravitacional. Esta faixa abrange os intervalos $n_{c} \simeq 9.4-22 \mathrm{~cm}^{-3} \mathrm{e}$ $R_{R S N} \simeq 46-84 \mathrm{pc}$. 

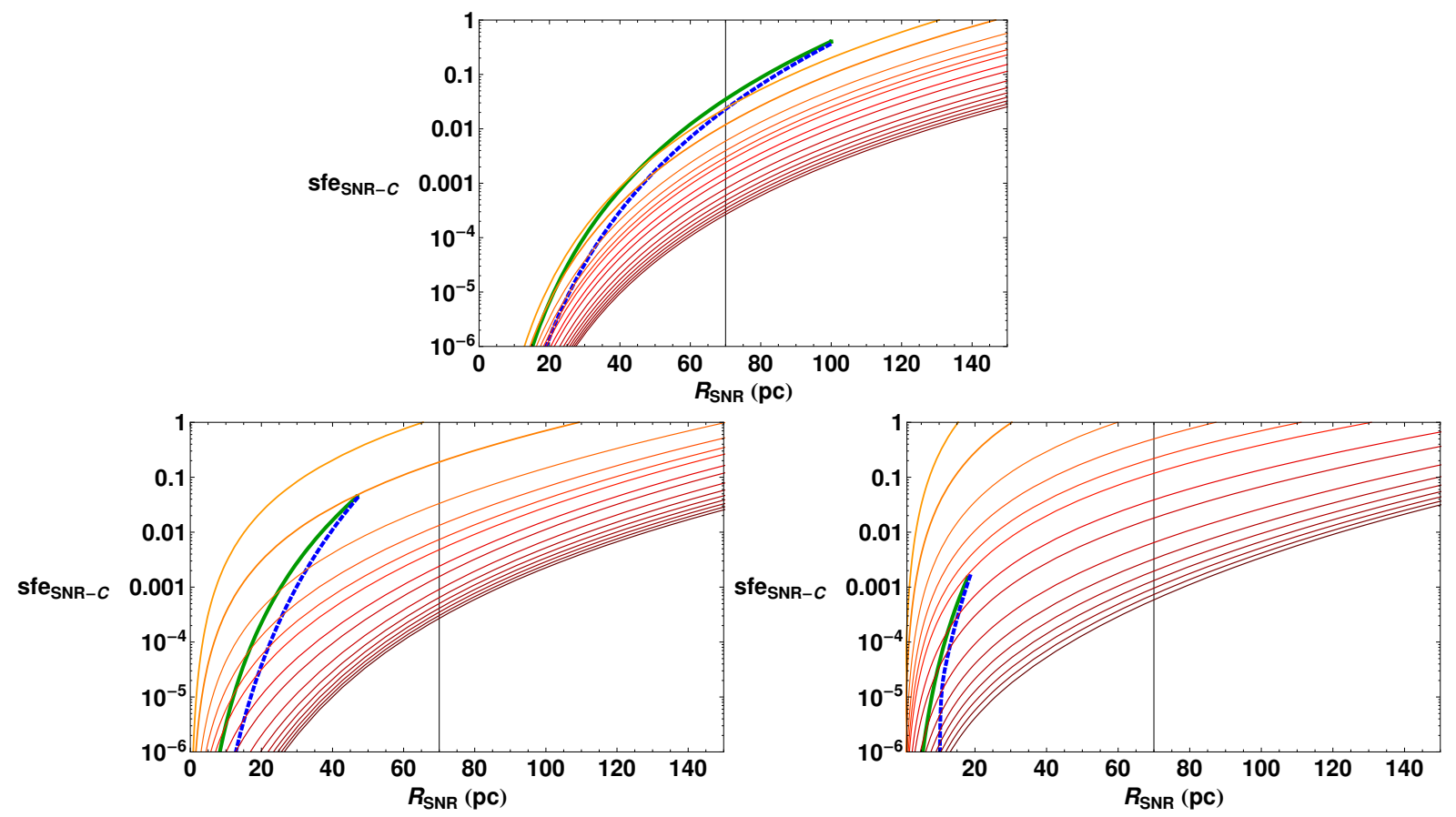

Figura 3.12: Gráficos da eficiência de formação estelar como uma função do raio do RSN no regime adiabático para vários valores da densidade da nuvem representados pelas linhas contínuas avermelhadas (de cima para baixo: 10, 20, 40, 60, 80, 100, 150, 200, 300, 400, 500, 600, 700, 800, $900 \mathrm{~cm}^{-3}$ ) para uma interação com uma nuvem com: (a) $B_{c}=0$ (painel superior); (b) $B_{c}=1 \mu \mathrm{G}$ (painel inferior à esquerda); e (c) $B_{c}=10 \mu \mathrm{G}$ (painel inferior à direita). As outras condições iniciais são $r_{c}=10 \mathrm{pc}, T_{c}=100 \mathrm{~K}$, e $\Lambda=5 \times 10^{-26} \mathrm{erg} \mathrm{cm}^{3} \mathrm{~s}^{-1}$. O meio ambiente possui $n=0.05 \mathrm{~cm}^{-3}$. O dois vínculos, isto é, a condição de não destruição da nuvem (linha sólida verde) e a condição de penetração do choque (linha pontilhada azul) delimitam a região permitida para a formação estelar nestes diagramas da SFE versus $R_{R S N}$. A linha contínua (preta) vertical representa o raio de transição do RSN do regime adiabático para o radiativo tal que somente os valores de $R_{R S N}$ no lado esquerdo desta linha são relevantes nestes diagramas (veja o texto para detalhes). 

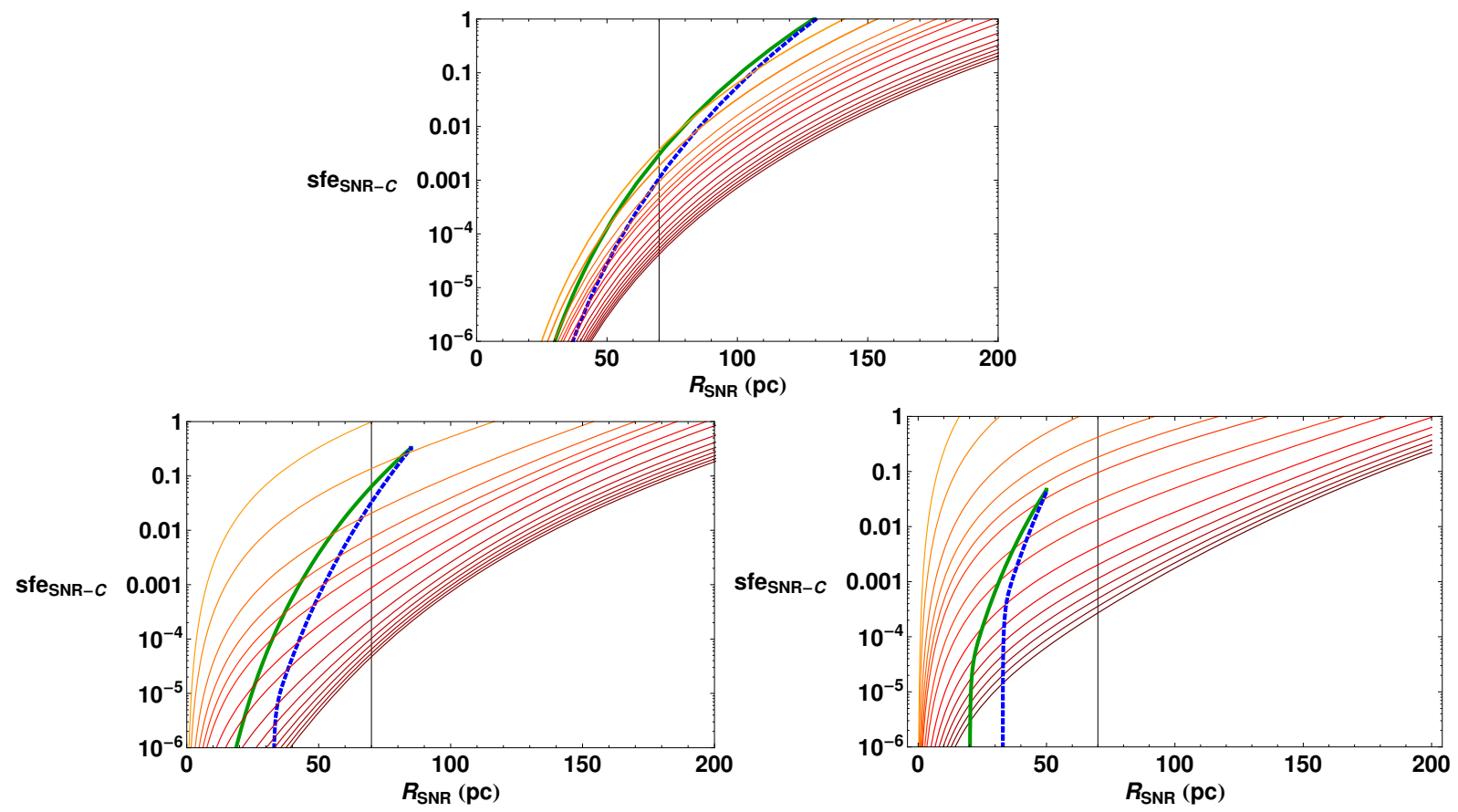

Figura 3.13: O mesmo que na Figura 3.12, mas agora considerando a interação com um RSN na fase radiativa. Nestes diagramas somente os valores de $R_{R S N}$ no lado direito da linha vertical preta são relevantes nesta fase. 


\section{Capítulo 4}

\section{Turbulência MHD e a difusão de campos magnéticos por reconexão}

magnética rápida em nuvens

\section{moleculares - Fundamentação teórica}

"If you want to learn about nature, to appreciate nature, it is necessary to understand the language that she speaks in." (Richard Feynman)

Neste Capítulo apresentaremos os fundamentos teóricos para a investigação de turbulência MHD em nuvens interestelares e os efeitos desta na difusão de campos magnéticos. Veremos que em presença de turbulência a reconexão das linhas de campo magnético torna-se rápida propiciando um transporte eficiente dos campos magnéticos. O coeficiente de difusão por reconexão turbulenta nos diferentes regimes de turbulência MHD sera também avaliado. Estes fundamentos serão em seguida testados numericamente no Capitulo 5. Verificaremos que ocorre uma eficiente difusão dos campos magnéticos por reconexão turbulenta, dos caroços para os envelopes das nuvens interestelares (das regiões mais densas, para as menos densas). Isso ajudara no processo de colapso gravitacional e na formação de estrelas no núcleo dessas nuvens, particularmente quando a turbulência 
for transônica e super ou trans-Alfvénica.

Como já discutimos nos Cap. 2, diversos mecanismos injetam energia no meio interestelar induzindo movimentos supersônicos em diversas escalas, desde de unidades astronômicas (U.A.) até as grandes escalas de kpc (kilo-parsecs) (Armstrong et al. 1995; Lazarian 2009). Candidatos sugeridos como mecanismos de injeção interna de turbulência incluem o "feedback" tanto de estrelas de baixa massa quanto de estrelas massivas. Este último é o maior agente estruturador no MIS (McCray \& Snow 1979), inicialmente através da produção de poderosos ventos e intensa radiação ionizante e, ao fim de suas vidas através de explosões como supernovas (SNs). Candidatos externos incluem choques espirais galáticos (Roberts 1969; Bonnell et al. 2006) e novamente as violentas explosões de supernovas (McKee \& Ostriker 1977; Wada \& Norman 2001; Elmegreen \& Scalo 2004; Melioli et al. 2006; Leão et al. 2009). Estes processos parecem ter energia suficiente para explicar a cinemática do MIS e podem gerar a relação escala de tamanho-velocidades de dispersão observada (Kornreich \& Scalo 2000). Portanto, o MIS é altamente turbulento o que muda a forma de estudarmos a dinâmica de muitos processos importantes que ocorrem nestes, incluindo a reconexão magnética.

A turbulência no MIS é observacionalmente revelada pelas flutuações de densidade do elétron, como podemos ver na Fig. 4.1. Esta Figura mostra a "'densidade da potência da turbulência"' em função do inverso da escala de comprimento das estruturas turbulentas, ou seja, o número de onda (Chepurnov \& Lazarian 2010). As linhas espectrais nos dão evidências observacionais mais diretas mostrando o espectro das flutuações de velocidade turbulenta supersônica (veja Padoan et al. 2004, 2009, Chepurnov et al. 2010).

O plasma magnetizado em meios astrofísicos apresenta números de Reynolds ${ }^{1}$ muito grandes devido às grandes escalas de comprimento envolvidas, permitindo o rápido desenvolvimento de instabilidades e movimentos turbulentos. Por exemplo, $R_{e} \sim 10^{10}$ ou maiores são muito comuns para fluidos astrofísicos. Os mecanismos de indução de turbulência injetam energia nas grandes escalas e então esta energia cascateia para as pequenas escalas através dos vórtices espalhando-se por todas as escalas.

\footnotetext{
${ }^{1} R_{e}=v L / \nu$, onde L e v são, respectivamente, a escala de comprimento e a velocidade do fluido, e $\nu$ sua viscosidade cinemática, veja Cap. 2
} 


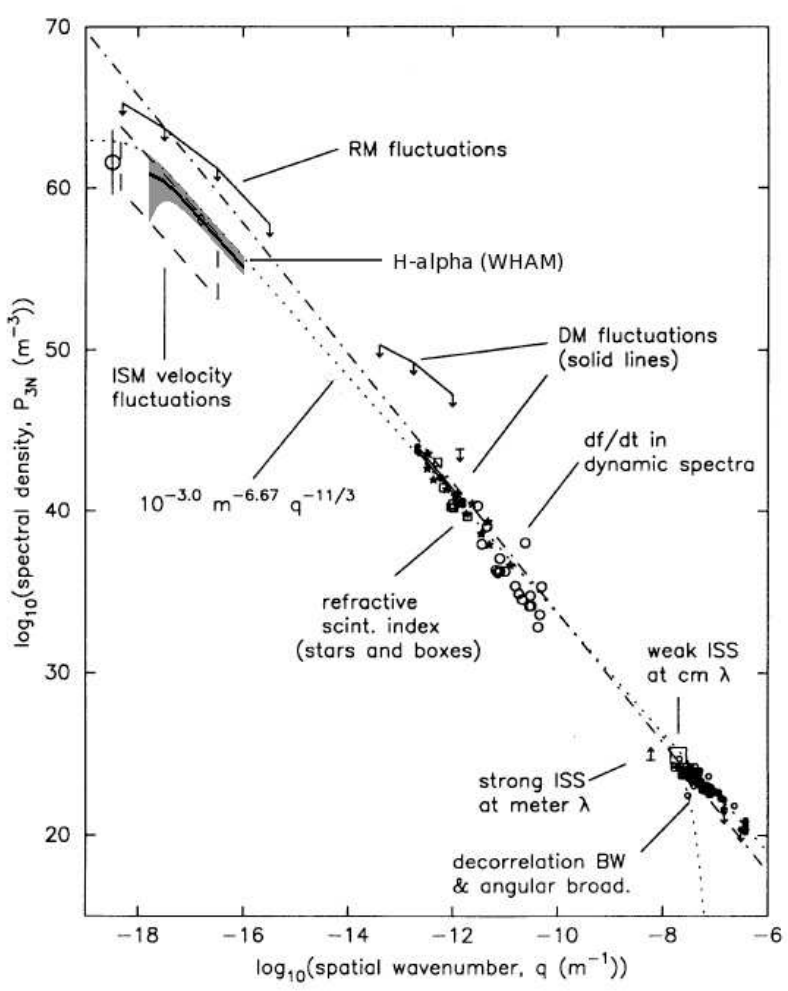

Figura 4.1: Turbulência no meio interestelar devido às flutuações de densidade dos elétrons usando os dados do WHAM (Wisconsin H $\alpha$ Mapper). A curva pontilhada corresponde à turbulência Kolmogorov (Chepurnov \& Lazarian 2010; Lazarian 2011)

Como turbulência está presente em todas as escalas do meio interestelar e em nuvens moleculares (Larson 1981, Armstrong et al. 1994, Elmegreen \& Falgarone 1996, Lazarian 2011 e referências ali contidas) podemos visualizar cada fase deste como parte da cascata turbulenta, isto é, como flutuações de densidade em um fluido compressível.

O verdadeiro papel que a turbulência e o campo magnético possuem no colapso de nuvens e na formação estelar é ainda bastante debatido. Uma questão importante neste debate é que, sob condições de MHD ideal, todo fluxo magnético deveria ser arrastado juntamente com o material colapsante. Neste caso, o campo magnético em uma protoestrela seria $\sim 10^{9} \mathrm{G}$, isto é, várias ordens de magnitude maior do que o observado em estrelas TTauri $\left(\lesssim 4 \times 10^{3}\right.$ G, veja Yang \& Johns-Krull 2011; Johns-Krull 2007). Este é comumente denominado de "problema do fluxo magnético" (veja Galli et al. 2006, JohnsKrull 2007, Santos-Lima et al. 2012). Para resolver este problema devem-se considerar 
mecanismos difusivos que possam remover fluxo magnético de uma nuvem colapsante e permitir a formação estelar, ao menos sob certas circunstâncias.

Para lidar com este problema de difusão de campo magnético em um meio interestelar parcialmente ionizado ou em nuvens moleculares, pesquisadores frequentemente invocam o mecanismo de difusão ambipolar (AD). A AD, primeiramente discutida no contexto de formação estelar por Mestel \& Spitzer (1956), tem sido extensivamente discutida desde então (e.g., Spitzer 1968; Nakano \& Tademaru 1972; Mouschovias 1976, 1977, 1979; Nakano \& Nakamura 1978; Shu 1983; Lizano \& Shu 1989; Fiedler \& Mouschovias 1992, 1993; Li et al. 2008; Fatuzzo \& Adams 2002; Zweibel 2002). Em princípio, a AD permite que fluxo magnético seja redistribuído durante o colapso de regiões de baixa ionização como resultado das colisões entre o gás neutro e o gás ionizado ao qual o campo magnético se acha acoplado.

Avanços recentes na teoria têm contudo questionado a eficiência deste processo de difusão em sistemas reais. Vários autores (Shu et al. 2006, Galli et al. 2006 Krasnopolsky et al. 2010, 2011) exploraram a fase de acreção em formação estelar de baixa massa e concluíram que deveria existir uma difusão efetiva cerca de três ordens de magnitude maior do que a difusividade Ôhmica para haver um transporte de fluxo magnético eficiente. Eles verificaram que a $\mathrm{AD}$ só poderia funcionar considerando-se apenas certos tamanhos de grãos de poeira na componente neutra do gás (veja também Li et al. 2011), o que restringiria a eficácia do mecanismo de $\mathrm{AD}$ a sistemas e condições muito especiais. Em outras palavras não existe ainda consenso se AD sozinha é eficiente o suficiente para resolver o problema de transporte de fluxo magnético em fluxos colapsantes e em nuvens moleculares.

Neste trabalho propomos uma solução diferente para o problema do fluxo magnético na formação estelar, um novo mecanismo para o transporte de fluxo magnético cujos fundamentos foram originalmente propostos por Lazarian (2005). Este baseia-se na teoria de reconexão magnética rápida em meios turbulentos de Lazarian \& Vishniac (2009; veja também Santos-Lima et al. 2010; de Gouveia Dal Pino et al. 2011; 2012; Santos-Lima, de Gouveia Dal Pino \& Lazarian 2012; Leão et al. 2012). A reconexão rápida do campo magnético em fluidos turbulentos permite ao campo mudar sua topologia (veja Fig. 4.2). 


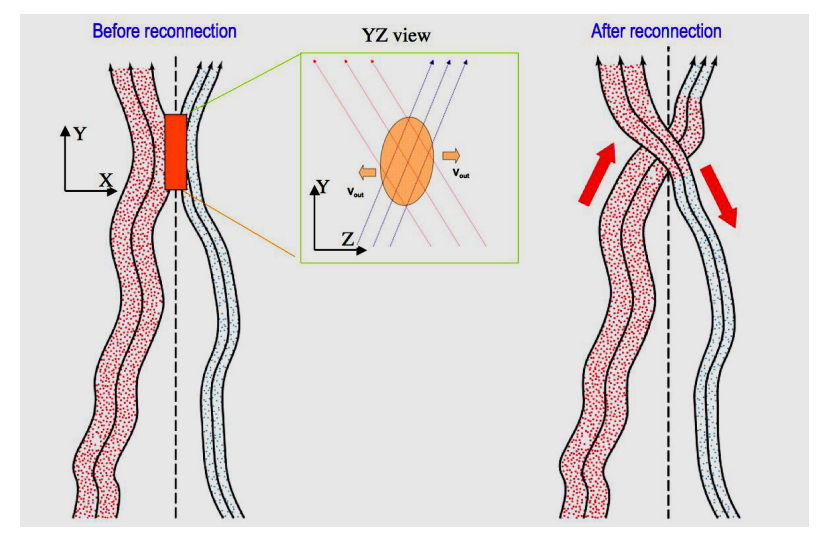

Figura 4.2: Movimento da matéria no processo de difusão de reconexão. Painel à esquerda: projeção xy antes da reconexão, o painel do meio mostra que os tubos de fluxo estão em ângulo no plano yz. Painel à direita: após a reconexão. De Lazarian (2011)

Como resultado o conceito tradicional de campos magnéticos congelados ao plasma não é mais aplicável. O plasma associado em um instante a um dado tubo de linhas de campo magnético, estará associado a outro tubo de linhas no instante seguinte (veja Figura 4.3). Do mesmo modo, pedaços de um tubo de linhas de campo magnético que pertenciam a um turbilhão de gás em um instante, são parcialmente transferidos para outros turbilhões nesse processo de difusão turbulenta. Esta difusão do plasma e do campo magnético é devida à reconexão e por isso é chamada de difusão por reconexão turbulenta. Ao mesmo tempo, a difusão por reconexão é independente da ionização, mas depende da escala dos vórtices turbulentos e das velocidades turbulentas. Em fluidos turbulentos, campos magnéticos sofrem contantes reconexões e mudam sua identidade todo o tempo. Isto implica que o campo magnético não é mais congelado a um fluido condutor perfeito se este fluido for turbulento (veja Vishniac \& Lazarian 1999; Eyink 2011; Lazarian et al. 2012)). Pode-se afirmar que o conceito de difusão por reconexão turbulenta estende o conceito de difusão turbulenta hidrodinâmica a fluidos magnetizados (Lazarian 2011).

Para quantificar a difusão por reconexão turbulenta precisamos de uma descrição quantitativa da turbulência MHD, o que faremos a seguir. Posteriormente, iremos explicar a física da difusão por reconexão tanto na escala macroscópica quanto do ponto de vista microscópico, ou seja, apelando para a reconexão de tubos de fluxo magnético e para a 


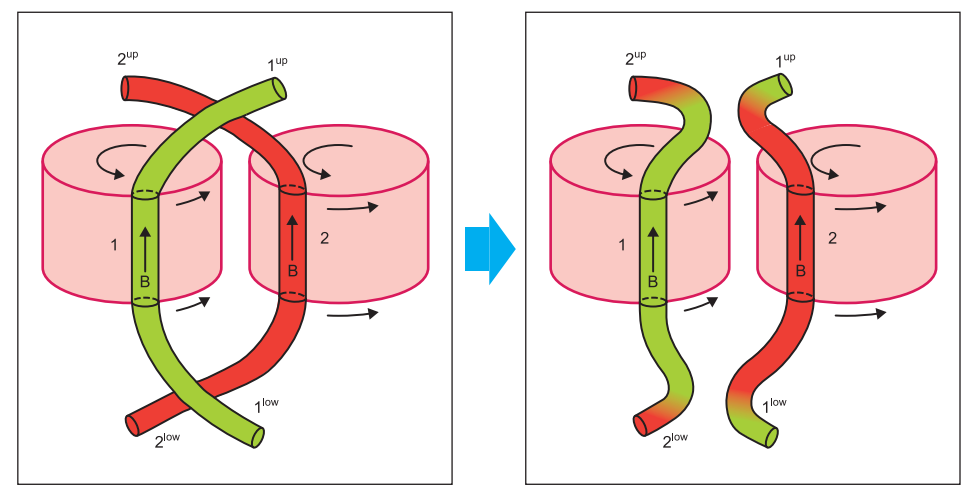

Figura 4.3: Difusão por reconexão. Ilustração da mistura de matéria e campo magnético devido à reconexão de dois tubos de fluxo de diferentes vórtices interagindo. De toda a escala de movimentos turbulentos apenas uma das escalas é apresentada. De Lazarian $(2011)$

difusão de linhas de campo magnético. Quantificaremos também a taxa de difusão por reconexão para a turbulência MHD fraca e forte.

\subsection{Turbulência MHD}

A história da teoria de turbulência MHD na última metade do século 20 foi de uma sucessão de tentativas de adaptar o modo de pensar da teoria de Kolmogorov de 1941 para turbulência hidrodinâmica (veja sessão 2.1.1 do Cap. 2) a fluidos magnetizados. Em um ambiente de plasmas magnetizados turbulentos, as suposições de isotropia e localidade de interações, apropriadas à turbulência hidrodinâmica, falham.

A turbulência MHD ainda carece de uma formulação teórica sólida. No entanto, uma formulação aproximada foi proposta por Goldreich \& Sridhar (1995; daqui por diante GS95). Por outro lado, esta tornou-se testável recentemente com o surgimento das simulações numéricas de alta resolução por meio de computação de alta performance. Como a teoria de fluidos estatística é bastante difícil de tratar analiticamente, um tratamento numérico buscando uma solução mais exata das equações de evolução temporal (considerando os efeitos numéricos) é frequentemente a única forma de se verificar a validade de um modelo analítico (Biskamp 2003). Assim, por exemplo, testes da teoria de 
turbulência MHD confirmaram as previsões de que vórtices magnetizados são alongados na direção do campo magnético (veja adiante) e forneceram resultados consistentes com as relações quantitativas do grau de elongação destes vórtices obtidas por Goldreich \& Sridhar (1995).

A turbulência MHD, quando suficientemente forte em termos da magnitude das interações não lineares entre as estruturas (veja descrição adiante), pode ser descrita por meio de vórtices ${ }^{2}$, de forma similar à turbulência hidrodinâmica. Por outro lado, ao contrário da turbulência de Kolmogorov, na presença de um campo magnético forte (dinamicamente importante) os vórtices tornam-se anisotrópicos. Os vórtices irão então misturar as linhas de campo na direção perpendicular ao campo magnético.

Considere um vórtice de dimensões $l_{\|}$e $l_{\perp}$, paralelo e perpendicular ao campo magnético, respectivamente. Por causa da transferência turbulenta para escalas perpendiculares menores, $l_{\perp}$ tende a encolher, tornando o vórtice mais alongado. Em geral, este processo será limitado pela propagação na direção paralela tal que (Biskamp 2003)

$$
\delta v_{l} / l_{\perp} \sim v_{A} / l_{\|}
$$

esta relação é conhecida como o balanço crítico (critical balance) de Goldreich \& Sridhar. Esta é a principal proposição da teoria de GS95, a igualdade entre um período de rotação do vórtice $l_{\perp} / \delta v_{l}$ e o período da correspondente onda de Alfvén $\sim l_{\|} / v_{A}$, onde $v_{A}$ é a velocidade de Alfvén. Então a cascata espectral ocorre principalmente no plano $k_{\perp}$ com o fluxo de energia $\epsilon$ constante no intervalo inercial (tal como na teoria de Kolmogorov para turbulência hidrodinâmica),

$$
\epsilon \sim v_{l}^{3} / l_{\perp}
$$

Usando a expressão anterior para $\delta v_{l}$ e combinando esta relação com a eq. 4.1 obtemos

\footnotetext{
${ }^{2} \mathrm{~A}$ descrição em termos de pacotes de onda interagentes ou modos é também possível com os correspondentes vetores de onda tendendo a ficar mais e mais perpendiculares ao campo magnético à medida que a cascata se desenvolve (Schekochihin \& Cowley 2007).
} 


$$
l_{\|} \sim \frac{v_{A}}{\epsilon^{1 / 3}} l_{\perp}^{2 / 3} \sim L^{1 / 3} l_{\perp}^{2 / 3}
$$

onde $L=v_{A}^{3} / \epsilon$. Encontramos que a anisotropia espectral aumenta com k, ou seja, com a diminuição do tamanho dos vórtices,

$$
k_{\perp} / k_{\|} \sim\left(L k_{\perp}\right)^{1 / 3}
$$

o que reflete a tendência de os vórtices tornarem-se mais e mais alongados com o cascateamento da energia para as menores escalas.

Esta relação de escala é confirmada pelas simulações numéricas (e.g., Cho \& Vishniac 2000; Beresnyak, Lazarian \& Cho 2005). A relação 4.2 é equivalente ao espectro de energia Kolmogorov perpendicular à direção do campo local:

$$
E_{k_{\perp}} \sim \epsilon^{2 / 3} k_{\perp}^{-5 / 3} \sim\left(v_{A}^{3} / L\right)^{2 / 3} k_{\perp}^{-5 / 3}
$$

O espectro de energia paralelo está atrelado ao espectro perpendicular. Assim, usando $k_{\perp}$ da eq. 4.3 temos

$$
E_{k_{\|}} \sim \epsilon^{3 / 2} v_{A}^{-5 / 2} k_{\|}^{-5 / 2}
$$

Observamos que se não existe campo médio a turbulência pode ser isotrópica com um espectro Kolmogorov visto que a contribuição da parte paralela é pequena no intervalo inercial e decai mais rapidamente. Na presença de um campo médio $B_{0}$, o espectro Kolmogorov, deve ser medido somente no plano perpendicular enquanto que a amplitude das flutuações paralelas ao campo são pequenas (Biskamp 2003).

É importante notar que as escalas $l_{\perp}$ e $l_{\|}$são medidas com respeito ao sistema de referência relacionado à direção do campo magnético local visto pelo vórtice.

A teoria GS95 descrita acima assume que a injeção de energia é isotrópica na escala $L$ e que a velocidade de injeção da turbulência $v_{L}$ é igual à velocidade de Alfvén no fluido $v_{A}$, isto é, o número de Mach Alfvénico é $M_{A} \equiv\left(v_{L} / v_{A}\right)=1$. Temos então uma descrição para a turbulência trans-Alfvénica. 
Este modelo foi posteriormente generalizado para os casos sub-Alfvénico, $M_{A}<1$, e super-Alfvénico, $M_{A}>1$ (veja Lazarian \& Vishniac 1999 e Lazarian 2006, respectivamente).

Se a turbulência é super-Alfvénica na escala de injeção $L$, i.e., se $M_{A}>1$ há uma outra escala, $l_{A}$, definida como a escala na qual o campo magnético torna-se dinamicamente importante, dada por

$$
l_{A}=L\left(v_{A} / v_{L}\right)^{3}=L M_{A}^{-3}
$$

na qual a velocidade turbulenta fica igual a $v_{A}$. Para escalas menores que $l_{A}$, a turbulência satisfaz o modelo de GS95. Já para $M_{A} \gg 1$, os campos magnéticos não são dinamicamente importantes nas maiores escalas e a turbulência nestas segue a cascata de Kolmogorov, sendo isotrópica $v_{l} \sim l^{1 / 3}$ em todo o intervalo de escalas compreendido entre $\left[L, l_{A}\right]$.

Se a turbulência é sub-Alfvénica na escala de injeção $L$, i.e., se $M_{A}<1$, ela será fraca no intervalo $\left[L, l_{\text {trans }}\right]$, onde $l_{\text {trans }}$ é definido como a escala abaixo da qual as interações deixam de ser fracas, dado por:

$$
l_{\text {trans }} \sim L\left(v_{L} / v_{A}\right)^{2} \equiv L M_{A}^{2}
$$

E assim, para escalas menores que $l_{\text {trans }}$, a turbulência irá obedecer à teoria GS95 (também denominada como turbulência MHD forte). Por outro lado, no regime de turbulência MHD fraca as perturbações propagam-se ao longo do campo médio como pacotes de onda MHD sofrendo muitas colisões antes de transferir energia para pequenas escalas. Por essa razão, nesse caso, a transferência de energia não é feita pelo cascateamento de turbilhões e portanto, não segue a teoria de Kolmogorov. Turbulência fraca, distintamente da forte, permite um tratamento analítico exato (Gaultier et al. 2000, Biskamp 2003, Schekochihin \& Cowley 2007). À medida que a turbulência fraca evolui, as interações dos pacotes de onda tornam-se mais fortes fazendo com que a turbulência torne-se forte.

Ao contrário da turbulência fraca, a turbulência forte é descrita por vórtices com o cascateamento acontecendo de forma similar à turbulência Kolmogorov, aproximada- 
mente dentro de um tempo de rotação do vórtice. Interações fortes entre pacotes de onda impedem o uso de uma aproximação perturbativa e não permitem uma derivação exata. Neste ponto, o uso de simulações numéricas é fundamental, conforme observamos anteriormente. Experimentos numéricos provaram o escalonamento previsto para turbulência MHD incompressível (veja Cho \& Vishniac 2000, Maron \& Goldreich 2001, Cho et al. 2002, Beresnyak \& Lazarian 2010, Beresnyak 2011) e para a componente Alfvénica da turbulência MHD em um fluido compressível (Cho \& Lazarian 2002, 2003, Kowal \& Lazarian 2010), onde simulações de Beresnyak et al. (2005) e Kowal, Lazarian \& Beresnyak (2007) demonstraram que o espectro de densidade torna-se mais suave e isotrópico com o aumento do número de Mach. A Tabela 4.1 apresenta um sumário dos diferentes regimes da turbulência MHD conforme descrito acima.

\section{Tabela 1}

Regimes e intervalos da turbulência MHD

\begin{tabular}{lcccc}
\hline \hline $\begin{array}{l}\text { Tipo } \\
\text { de turbulência MHD }\end{array}$ & $\begin{array}{c}\text { Velocidade } \\
\text { de injeção }\end{array}$ & $\begin{array}{c}\text { Intervalo } \\
\text { de escalas }\end{array}$ & $\begin{array}{c}\text { Tipo de } \\
\text { movimento }\end{array}$ & $\begin{array}{c}\text { Formas } \\
\text { de estudo }\end{array}$ \\
\hline Fraca & $v_{i n j}<v_{A}$ & {$\left[L, l_{\text {trans }}\right]$} & onda & analítico \\
\hline Forte & & & & \\
sub-Alfvénica & $v_{i n j}<v_{A}$ & {$\left[l_{\text {trans }}, l_{\text {min }}\right]$} & vórtices & numérico \\
\hline Forte & & & & \\
super-Alfvénica & $v_{i n j}>v_{A}$ & {$\left[l_{A}, l_{\text {min }}\right]$} & vórtices & numérico \\
\hline
\end{tabular}

$L$ e $l_{\min }$ são as escalas de injeção e de dissipação

$l_{\text {trans }}$ e $l_{A}$ são dadas pelas Eq. (4.8) e Eq. (4.7), respectivamente. 


\subsection{Reconexão magnética rápida em fluidos turbu- lentos}

Para explicar a física da difusão por reconexão magnética turbulenta e sua conexão com a formação estelar precisamos compreender antes o mecanismo de reconexão magnética. O primeiro modelo de reconexão construído para um fluido laminar (não turbulento), foi proposto por Parker (1957) e Sweet (1958).

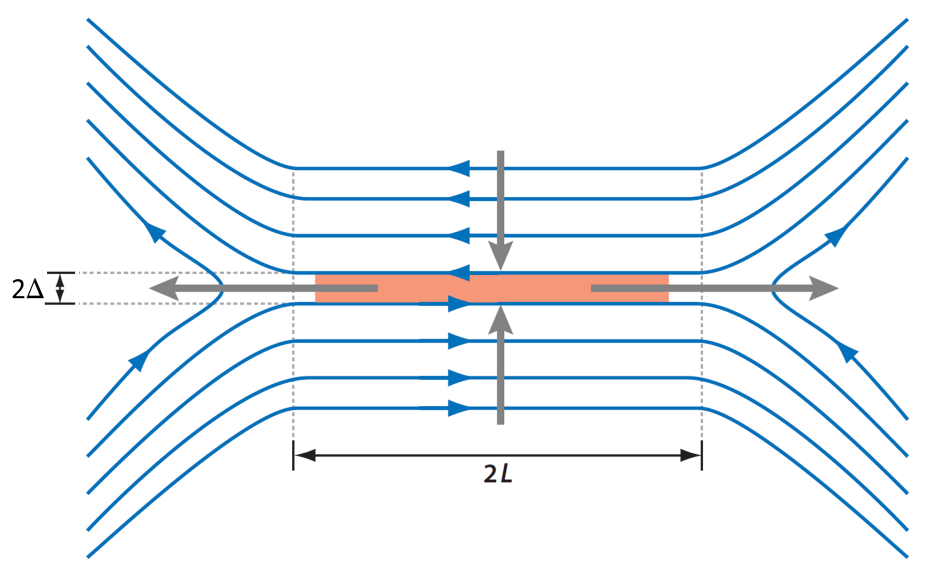

Figura 4.4: Esquema da geometria das linhas de campo magnético na reconexão de SweetParker. As linhas de campo magnético opostamente direcionadas fluem para uma região de comprimento $2 L$ e reconectam em uma camada de difusão com largura $2 \Delta$ (Adaptado de Zweibel \& Yamada 2009).

Dois fluxos de campos magnéticos opostamente direcionados, $\pm B$, em um plasma com densidade $\rho$ e condutividade $\sigma$ fluem para uma interface neutra à velocidade $v_{\text {in }}$ com uma extensão $2 L$ e espessura $2 \Delta$ (veja a Figura 4.4) onde a reconexão (i.e., aniquilação parcial das linhas) ocorre nessa interface. A Figura 4.4 apresenta um corte de uma camada de reconexão em 3D. Apenas uma componente do campo magnético é apresentada da configuração tridimensional da reconexão de tubos de fluxo magnético. Esta figura ilustra porque a reconexão é tão lenta nesse modelo de Sweet-Parker, que considera campos magnéticos como laminares e portanto, a condição de congelamento do campo magnético ao gás é violada somente na fina interface onde a resistividade do plasma domina. As 
escalas onde a difusão resistiva é importante são microscópicas e portanto esta camada é muito fina, ou seja, $2 \Delta \ll 2 L$. O campo reconectado e o plasma são então expelidos a uma velocidade $v_{\text {out }}$ na direção perpendicular à de entrada (veja figura 4.4). Assume-se que o sistema é estacionário. A teoria de Sweet-Parker prevê uma taxa de reconexão $v_{r e c}=v_{i n}$ e usando a geometria acima e conservação de momentum é fácil demonstrar que:

$$
v_{r e c, S P}=v_{A} / \sqrt{S}
$$

onde o número de Lundquist, S, é a razão entre o tempo de difusão ôhmico $t_{d i f f} \equiv L^{2} / \eta$ o tempo de Alfvén $t_{A} \equiv L / v_{A}$ e é dado por

$$
S=L v_{A} / \eta
$$

Como a maiorias dos sistemas astrofísicos possui números de Lundquist muito grandes a reconexão nestes é muito lenta. É também fácil ver que a difusão de Sweet-Parker é lenta pois todo o fluido que entra na região de reconexão deve sair através de uma interface resistiva muito fina.

Procurando tratar tanto de plasmas colisionais quanto acolisionais, Lazarian \& Vishniac (1999) propuseram um modelo de reconexão rápida na presença de turbulência MHD.

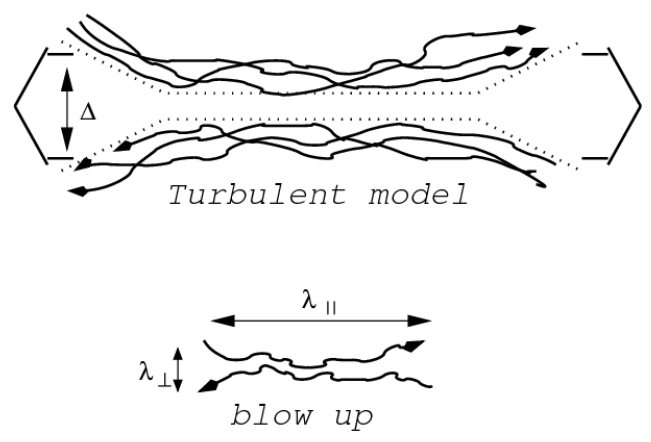

Figura 4.5: Painel superior: reconexão de acordo com o modelo de LV99. $\Delta$ é determinado pela turbulência e pode ser grande. Painel inferior: reconexão de campo magnético em pequenas escalas. Adaptado de Lazarian, Vishniac \& Cho (2004).

O modelo de Lazarian \& Vishniac, LV99, generaliza o modelo de Sweet-Parker con- 
siderando a estocacidade das linhas de campo magnético (painel inferior da Figura 4.5). A presença de turbulência permite que muitas linhas de campo entrem na região de reconexão simultaneamente. Portanto dependendo do nível da turbulência a taxa de reconexão pode tornar-se bem alta. De fato, é possível demonstrar que nesse caso a taxa de reconexão magnética é (LV99; veja também Eyink et al. 2011)

$$
v_{r e c, L V 99} \approx v_{A}\left(L / l_{i n j}\right)^{1 / 2} M_{A}^{2}
$$

no limite onde $L \leqslant l_{i n j}$, onde $l_{i n j}$ é a escala de injeção da turbulência. Esta é bem mais rápida que a prevista pelo modelo de Sweet-Parker e independente da resistividade microscópica Ôhmica. As previsões dessa teoria foram testadas com sucesso com simulações numérica magneto-hidrodinâmicas 3D por Kowal et al. (2009) (veja também Lazarian et al. 2010).

Os resultados acima demonstram que em presença de turbulência, a reconexão das linhas de campo magnético deve ser rápida. Conforme discutimos na sessão 4.1, a mistura devida aos vórtices turbulentos na direção perpendicular ao campo magnético local induz uma difusão turbulenta similar à de vórtices hidrodinâmicos. Isto contraria o conceito clássico de congelamento perfeito entre as linhas de campo magnético. Ao mesmo tempo, os movimentos de mistura em turbulência MHD exigem que eventos rápidos de reconexão ocorram a cada rotação do vórtice (Lazarian 2011). Nas pequenas escalas as linhas do campo magnético são quase paralelas e quando elas se cruzam o gradiente de pressão não é $v_{A}^{2} / l_{\|}$, mas $\operatorname{sim}\left(l_{\perp}^{2} / l_{\|}^{3}\right) v_{A}^{2}$, uma vez que apenas a energia da componente do campo magnético que não é cascateada está disponível (Lazarian 2011; Eyink et al. 2012). Por outro lado, a contração do comprimento característico de uma dada linha de campo devido à reconexão entre vórtices vizinhos é $l_{\perp}^{2} / l_{\|}$, o que dá uma taxa de ejeção efetiva de $v_{A} / l_{\|}$. Como a largura da camada de difusão sobre o comprimento $l_{\|}$, é $l_{\perp}$, a Eq.(4.11) deverá ser substituída por $v_{R} \approx v_{A}\left(l_{\perp} / l_{\|}\right)$, a qual dá a taxa de reconexão $v_{A} / l_{\|}$, que é a taxa de cascateamento não-linear na escala $l_{\|}$. Isso garante auto-consistência para fluidos com turbulência MHD forte e altamente condutores (LV99). Se não houvesse reconexão ocorreria o acúmulo de nós magnéticos não resolvidos, achatando o espectro da turbulência 
nas pequenas escalas. Isso porém, contradiz tanto as medições da turbulência do vento solar quanto os cálculos numéricos.

\subsection{Coeficiente de difusão por reconexão magnética turbulenta}

Para calcular o coeficiente de difusão por reconexão turbulenta, vamos considerar a taxa máxima permitida, ou seja, vamos avaliar a difusividade dos maiores turbilhões. Primeiramente, ao lidarmos com a difusão por reconexão magnética devemos considerar todos os regimes da turbulência MHD, super-Alfvénico, trans-Alfvénico e sub-Alfvénico. Comecemos com um regime super-Alfvénico, isto é, $M_{A}>1$. O campo magnético só fica dinamicamente importante nas escalas em que sua energia excede a dos vórtices isto e, quando a velocidade Alfvénica torna-se maior do que a velocidade dos vórtices. Como vimos, essa é a escala $l_{A}$ (eq. 4.7) na qual o campo magnético torna-se dinamicamente importante e a natureza da turbulência muda de hidrodinâmica para MHD (ver Lazarian 2006),

Se o caminho livre médio das partículas do fluido é maior do que $l_{A}$, a escala $l_{A}$ deve agir como um livre caminho médio efetivo em termos da difusão de partículas ao longo do campo magnético. Isto é importante para a difusão de calor mas não para a difusão de campo magnético induzida por turbulência (Lazarian 2011). Nesse caso, o coeficiente de difusão nas grandes escalas induzido pela turbulência com uma escala de injeção $L$ e velocidade $v_{L}$ coincide com o coeficiente de difusão para a turbulência hidrodinâmica:

$$
\kappa_{\text {sup } A} \simeq L v_{L}
$$

Isso ocorre pois os maiores vórtices da turbulência super-Alfvénica quase não são afetados pelo campo magnético.

Para turbulência sub-Alfvénica na escala de injeção, i.e., $v_{A} \gg v_{L}$, nas grandes escalas a turbulência é fraca e os campos magnéticos são levemente perturbados pela propagação de ondas Alfvén. Os pacotes de onda, à medida que evoluem mudam sua escala perpendi- 
cular ao campo, $l_{\perp}$, enquanto que sua escala $l_{\|}$, ao longo do campo magnético não muda. A difusão induzida por turbulência fraca é pequena quando comparada à turbulência hidrodinâmica e pode ser estimada como

$$
\kappa_{\text {fraca }} \sim d^{2} \omega
$$

onde $d$ é o passeio aleatório ("random walk") da linha de campo em um período de onda $\sim \omega^{-1}$. A turbulência fraca na escala $l_{i n j}$ evolui em um tempo de evolução não linear (veja Cho, Lazarian \& Vishniac 2003)

$$
\tau \sim\left(v_{A} / v_{L}\right)^{2} \omega^{-1}
$$

O deslocamento das linhas de campo magnético na direção perpendicular é um resultado do passeio aleatório

$$
\left\langle y^{2}\right\rangle \sim(\tau \omega) d^{2}
$$

De acordo com LV99, o deslocamento das linhas de campo magnético em uma distância $x$ é um passeio aleatório espacial dado por $d\left\langle y^{2}\right\rangle / d x \sim l_{\text {inj }}\left(v_{L} / v_{A}\right)^{4}$, que resulta em

$$
\left\langle y^{2}\right\rangle \sim L x\left(v_{L} / v_{A}\right)^{4}
$$

Combinando as Eqs. (4.13), (4.14), (4.15) and (4.16) obtemos o coeficiente de difusão para a turbulência fraca (sub-Alfvénica) (Santos-Lima et al. 2010; Lazarian 2011)

$$
\kappa_{\text {fraca }} \sim L v_{L}\left(v_{L} / v_{A}\right)^{3} \equiv L v_{L} M_{A}^{3}
$$

o qual é menor do que sua contrapartida hidrodinâmica por um fator $M_{A}^{3} \ll 1$.

A contribuição adicional para a difusividade no caso $v_{A} \gg v_{L}$ vem das pequenas escalas nas quais a turbulência magnética torna-se forte, isto é, na escala $l_{\text {trans }}$ (definida anteriormente (eq. 4.8)) na qual a condição de balanço crítico $l_{\|} / v_{A} \approx l_{\perp} / \delta v_{l}$ é satisfeita, tornando a turbulência forte. Assim a velocidade correspondente a $l_{\text {trans }}$ será $v_{\text {trans }} \sim$ $v_{L}\left(v_{L} / v_{A}\right)$. Para a turbulência forte a difusão será 


$$
\kappa_{\text {forte }} \sim v_{\text {trans }} l_{\text {trans }} \sim L v_{L}\left(v_{L} / v_{A}\right)^{3}
$$

o que coincide com a Eq. (4.17), indicando que o aumento da difusividade de pequenos vórtices no regime de turbulência MHD forte pode produzir uma mistura eficiente do campo magnético da mesma forma que a difusividade induzida pela turbulência fraca na escala de injeção. Este resultado mostra a conexão entre a turbulência fraca e a forte em termos da mistura que estes processos induzem.

O caso trans-Alfvénico $\left(M_{A} \sim 1\right)$ é equivalente a substituir $M_{A} \equiv 1$ nas expressões obtidas para os casos super-Alfvénico ou sub-Alfvénico, ou seja, na Eq. (4.17) ou (4.18).

As eqs. (4.12) e (4.17) e (4.18) para a difusão de campos magnéticos por reconexão turbulenta serão testadas no estudo numérico que apresentaremos no próximo capítulo acerca dos efeitos da turbulência MHD na formação estelar em nuvens moleculares. Nesses casos, poderemos identificar a escala de injeção $l_{i n j}$ e a velocidade turbulenta $v_{\text {turb }}$ com a dimensão $l_{\text {nuvem }}$ e a velocidade de dispersão medida na nuvem $v_{\text {nuvem }}$, respectivamente. No caso de turbulência sub-Alfvénica, o coeficiente de difusão pode ser aproximado por

$$
\kappa_{\text {nuvem }} \sim v_{\text {nuvem }} l_{\text {nuvem }}\left(v_{\text {nuvem }} / v_{A}\right)^{3}
$$

enquanto que para o caso da turbulência trans-Alfvénica ou super-Alfvénica podemos usar a mesma estimativa mas sem o fator $M_{A}^{3}$. 


\section{Capítulo 5}

\section{Formação estelar em nuvens turbulentas e o transporte de Fluxo}

\section{Magnético}

"Everthing should be made as simple as possible, but not simpler" (Albert Einstein)

Conforme vimos nos Caps. 1 e 4, para um glóbulo de nuvem molecular formar estrelas deve haver transporte de fluxo magnético das regiões densas mais internas para as regiões menos densas mais externas da nuvem, de outra forma o colapso poderá ser impedido pela força magnética. Em um estudo recente Santos-Lima et al. (2010) mostraram numericamente que o mecanismo baseado em reconexão magnética turbulenta rápida que descrevemos no Cap. 4 pode fornecer um transporte eficiente de campo magnético para fora do núcleo da nuvem. Esse estudo numérico considerou por simplicidade uma geometria cilíndrica para as nuvens. Aqui consideramos nuvens e glóbulos mais realistas com potenciais gravitacionais esféricos devido a estrelas embebidas na nuvem e também levando em conta os efeitos da auto-gravidade do gás. Estudamos tanto nuvens começando em equilíbrio magneto-hidrostático quanto nuvens colapsantes.

Neste capítulo, apresentaremos os resultados desse estudo. Veremos que os mesmos confirmam os resultados do estudo de Santos-Lima et al. (2010), embora o transporte 
de fluxo magnético seja menos eficiente na presença de um potencial esférico. Obtivemos ainda as condições para as quais o transporte por reconexão turbulenta torna-se eficiente o bastante para tornar uma nuvem inicialmente subcrítica uma nuvem supercrítica e colapsante. Nossos resultados indicam que a formação de um núcleo supercrítico é resultado de uma complexa interação entre a gravidade, auto-gravidade, a intensidade do campo magnético e uma turbulência aproximadamente trans-sônica e trans-Alfvénica. Em particular, a auto-gravidade favorece a difusão do campo magnético por reconexão turbulenta e seu desacoplamento do gás colapsante, em geral, de forma mais eficiente do que um campo gravitacional externo. Verificamos que a reconexão magnética turbulenta é geralmente capaz de remover fluxo magnético da maioria dos glóbulos colapsantes testados aqui, porém somente uns poucos desenvolvem núcleos críticos ou super-críticos, o que é compatível com a baixa eficiência de formação estelar esperada das observações. A formação destes núcleos super-críticos devido ao transporte de fluxo magnético por reconexão turbulenta é restrita, em nossos testes, à seguinte faixa de condições iniciais para as nuvens: razão pressão magnética-pressão térmica, $\beta \sim 1$ a 3 , razões entre a energia turbulenta e a energia magnética $E_{\text {turb }} / E_{\text {mag }} \sim 1.62$ to 2.96 , e densidades $50<n<140 \mathrm{~cm}^{-3}$ quando consideramos uma massa média em estrelas embebidas $\mathrm{M}_{\star} \sim 25 \mathrm{M}_{\odot}$, implicando uma massa total da nuvem (gás + estrelas) $\mathrm{M}_{\text {tot }} \lesssim 120 \mathrm{M}_{\odot}$.

\subsection{Domínio Computacional e Metodologia Numérica}

O ambiente astrofísico que investigamos neste trabalho consiste de uma nuvem molecular inicialmente subcrítica, ou mais especificamente, um glóbulo de nuvem molecular de alta densidade com um pequeno grupo de estrelas embebidas, sustentado por campo magnético e turbulência. O objetivo é examinar por meio de simulações numéricas magneto-hidrodinâmicas (MHD) tri-dimensionais (3D), as condições em que sob as quais o transporte de fluxo magnético por reconexão turbulenta permitirá a contração do gás auto-gravitante para formar um caroço supercrítico. Também consideramos alguns modelos sem incluir auto-gravidade de forma a comparar com o trabalho anterior de SantosLima et al. (2010). 
Na maioria de nossos experimentos numéricos o sistema começa já fora de equilíbrio entre a gravidade e as outras forças. Um glóbulo inicialmente esférico de gás com um potencial gravitacional central que simula um pequeno grupo de estrelas embebidas é colocado no centro de um meio inicialmente homogêneo magnetizado. Segue-se então uma violenta contração do gás por um curto período de tempo (da ordem do tempo de queda-livre), após o qual, devido à presença do campo magnético e da turbulência injetada, o sistema evolui mais suavemente. Como exemplo, a Figura 5.1 mostra o mapa de densidade logarítmico do corte central do modelo N2b (sem turbulência injetada) logo após essa rápida contração inicial ( 1.1 Myrs). A configuração do campo magnético está sobreposta ao mapa de densidade. Note-se o achatamento da nuvem devido à presença do campo magnético vertical.

Entre os modelos estudados, consideramos também uma nuvem começando em equilíbrio magneto-hidrostático, para comparação com os outros modelos. (Neste caso, começamos com uma nuvem com densidade uniforme e a deixamos entrar em equilíbrio e só então injetamos a turbulência).

Cada sistema é simulado dentro de um domínio cúbico com fronteiras periódicas. Como uma nuvem molecular gigante típica possui vários glóbulos em seu interior o uso de fronteiras periódicas para modelar um desses glóbulos é bastante apropriado. Por simplicidade empregamos uma equação isotérmica de estado, com uma mesma temperatura para todo o sistema, o que significa que o sistema irradia eficientemente (veja Cap. 2).

Escolhemos valores característicos para os parâmetros físicos de nossos modelos baseados em dados observacionais (veja, e.g. Mac Low \& Klessen 2004). ${ }^{1}$ O domínio computacional possui lados $L=3.25$ pc. Para uma resolução de $256^{3}$ o comprimento correspondente da célula é $\approx 0.013$ pc $\sim 2600$ AU. No estudo numérico de transporte por reconexão turbulenta MHD 3D em nuvens cilíndricas realizado por Santos-Lima et al. (2010), testes com diferentes resoluções entre $128^{3}$, e $512^{3}$ foram realizados e produziram essencialmente os mesmos resultados, portanto na maioria dos modelos simulados aqui

\footnotetext{
${ }^{1}$ Notamos que neste estudo, uma vez que consideramos a auto-gravidade, os resultados não são independentes da escala, e portanto um espaço de parâmetros com condições iniciais físicas definidas a priori deve ser considerado.
} 


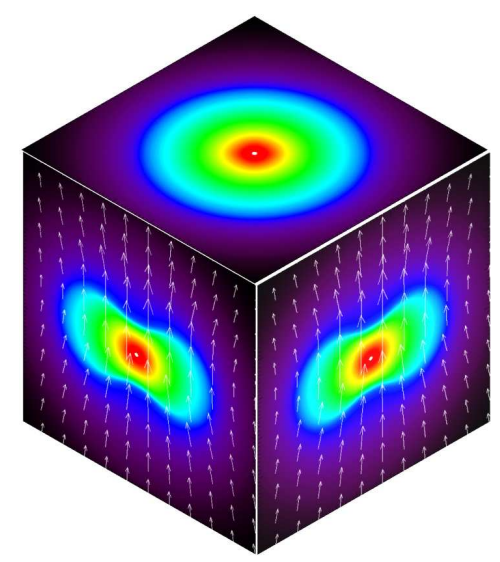

Figura 5.1: Examplo de uma nuvem no domínio computacional (sem turbulência injetada) logo após a rápida contração inicial em 1.1 Myrs. Mapa de densidade logarítmico e vetores de campo magnético do corte central (projetado nas paredes do domínio computacional cúbico) do modelo N2b da Tabela 5.1 com $M_{\text {pot }}=40.7 \mathrm{M}_{\odot}$, densidade inicial $n_{0}=90 \mathrm{~cm}^{-3}$, e $\beta=3.0$.

usamos uma resolução de $256^{3}$ (veja Fig. 5.9 a seguir).

A velocidade isotérmica do som considerada em todos os modelos é $c_{s}=3 \times 10^{4}$ $\mathrm{cm} / \mathrm{s}$, a qual corresponde a uma temperatura $T=10.9 \mathrm{~K}$ (para um peso molecular médio $\mu=1.0)$. Para cobrir um espaço de parâmetros tão apropriado quanto possível para as condições observadas consideramos uma densidade inicial uniforme do gás, $\rho_{0}$, entre $1.67 \times 10^{-23} \mathrm{~g} \mathrm{~cm}^{-3}\left(\right.$ ou $\left.n_{0}=10 \mathrm{~cm}^{-3}\right)$ e $1.67 \times 10^{-22} \mathrm{~g} \mathrm{~cm}^{-3}\left(\right.$ ou $\left.n_{0}=100 \mathrm{~cm}^{-3}\right)$. Também consideramos valores iniciais da razão entre as pressões térmica e magnética, $\beta=c_{s}^{2} \rho /\left(B^{2} / 8 \pi\right)=3.0,1.0$ e 0.3. Isto implica velocidades de Alfvén $v_{A} \approx 2.4 \times 10^{4} \mathrm{~cm} / \mathrm{s}$, $4.2 \times 10^{4} \mathrm{~cm} / \mathrm{s}$, e $7.8 \times 10^{4} \mathrm{~cm} / \mathrm{s}$, respectivamente, e intensidades do campo magnético inicial entre $B_{0}=0.35 \mu G$ e $3.4 \mu G$.

A massa inicial de estrelas imersas no glóbulo de nuvem $M_{\star}$ também foi variada entre $20-60 \mathrm{M}_{\odot}$. Estas estrelas produzem um potencial gravitacional esférico $\Psi_{\star}(r)$, dado por:

$$
\Psi_{\star}\left(r \leq r_{\max }\right)=-\frac{G M_{\star}}{\left(r+r_{\star}\right)}
$$

onde $r$ é a distância ao centro da caixa computacional, e $r_{\max } \sim 1.45$ pc é um raio de 
corte (cut-off) na força gravitacional para assegurar a simetria visto que usamos fronteiras periódicas, e $r_{\star}$ é um raio de suavização. Seu valor dá o comprimento característico do glóbulo. Fixamos $r_{\star}=0.325 \mathrm{pc}$ de modo a limitar o valor da força gravitacional e assegurar que o sistema não fosse instável a Parker-Rayleigh-Taylor.

Para seguir a evolução de uma nuvem integramos numericamente as equações da magneto-hidrodinâmica (MHD) em sua forma ideal:

$$
\begin{gathered}
\frac{\partial \rho}{\partial t}+\nabla \cdot(\rho \mathbf{v})=0 \\
\rho\left(\frac{\partial}{\partial t}+\mathbf{v} \cdot \nabla\right) \mathbf{v}=-c_{s}^{2} \nabla \rho+\frac{1}{4 \pi}(\nabla \times \mathbf{B}) \times \mathbf{B} \\
\\
-\rho \nabla\left(\Psi_{\text {gas }}+\Psi_{\star}\right)+\mathbf{f} \\
\frac{\partial \mathbf{B}}{\partial t}=\nabla \times(\mathbf{v} \times \mathbf{B})
\end{gathered}
$$

onde as variáveis independentes possuem suas definições usuais, isto é, $\rho$ é a densidade volumétrica, v é velocidade, e $\mathbf{B}$ é o campo magnético (veja também Ap. B). O campo magnético $\mathbf{B}$, satisfaz a condição de divergência $\nabla \cdot \mathbf{B}=0$. O potencial $\Psi_{\text {gas }}$ é devido à auto-gravidade do gás que obedece à equação de Poisson:

$$
\nabla^{2} \Psi_{g a s}=4 \pi G \rho
$$

O termo fonte $\mathbf{f}$ na equação 5.3 é a força responsável pela injeção de turbulência. Empregamos um 'forcing' isotrópico, não-helicoidal, solenoidal, com correlação delta no tempo $f$. Este induz um campo de velocidades isotropicamente distribuído, concentrado ao redor de um comprimento de onda típico que define a escala de injeção $l_{i n j}$. A potência injetada é constante, o que mantém a velocidade randômica do gás $v_{r m s}$ aproximadamente contante. Em todos os modelos com injeção de turbulência usamos $l_{i n j} \approx 1.3$ pc e $v_{r m s}$ entre $2.4 \times 10^{4}$ e $5.7 \times 10^{4} \mathrm{~cm} / \mathrm{s}$. A injeção de energia turbulenta começa em $t=0.0 \mathrm{Myr}$ e esta evolui lentamente até desenvolver-se completamente em $t \sim 10.6$ Myrs.

Empregamos uma versão do código cartesiano Godunov-MHD, originalmente desenvolvido por G. Kowal (Kowal et al. 2007, veja também, Falceta-Gonçalves et al. 2008; 
Santos-Lima et al. (2010)), usando um resolvedor HLL e Runge-Kutta de segunda ordem para a integração temporal. A equação de Poisson é resolvida por um algorítimo baseado em multigrid (Press, Teukolsky \& Flannery, 1978). Veja uma descrição mais detalha do código numérico no Ap. B.

\subsection{Condições Iniciais}

Na Tabela 5.1 temos as condições iniciais usadas para todos os modelos simulados com e sem auto-gravidade. Os modelos sem auto-gravidade são denominados "R", os autogravitantes começando fora do equilíbrio são chamados "N"e o modelo começando em equilíbrio magneto-hidrostático é denominado modelo "E". Os modelos "R", embora sejam independentes da escala são também descritos aqui por suas condições iniciais em unidades físicas para comparação com os outros modelos, exceto pelo parâmetro adimensional $A$ que mede a força do potencial gravitacional $A=\left(G M_{\star}\right) /\left(L c_{s}^{2}\right)$, onde $\mathrm{G}$ é a constante gravitacional de Newton, $M_{\star}$ é a massa do potencial estelar, L é o tamanho do domínio computacional (que também dá a unidade de distância do código), e $c_{s}$ é a velocidade do som (que é também a unidade de velocidade no código). Os outros parâmetros na Tabela são a massa total $M_{t o t}$, isto é, a soma das massas das estrelas e do gás, a densidade numérica inicial gasosa da nuvem $n_{0}$, a qual para o modelo em equilíbrio refere-se à densidade central inicial do gás da nuvem, o campo magnético inicial $B_{0}$ admitido uniforme, a razão pressão térmica-pressão magnética $\beta$, a velocidade turbulenta $v_{\text {turb }}$, a velocidade de Alfvén $v_{A}$, a massa de Jeans inicial da nuvem em presença de campo magnético $m_{J, B}$, e a razão entre as densidades iniciais de energia turbulenta e magnética $E_{\text {turb }} / E_{\text {mag }}$ para o núcleo $\left(r_{c} \leq 0.3 \mathrm{pc}\right)$. As velocidades turbulenta e de Alfvén são dadas em unidades da velocidade do som isotérmica $c_{s}=3 \times 10^{4} \mathrm{~cm} / \mathrm{s}$.

A Tabela 5.2 apresenta o tempo dinâmico, $t_{d y n}=L / c_{s}$. Este pode ser comparado com o tempo de queda-livre do gás da nuvem, dado por,

$$
t_{f f}=\sqrt{\frac{3 \pi}{32 G \rho}}
$$

e ao tempo de difusão do campo magnético, $t_{\text {diff }}$, 
Tabela 5.1: Condições iniciais para os modelos colapsantes.

\begin{tabular}{lccccccccc}
\hline \hline & & & & & & & & \\
Modelo & $M_{\star}\left(\mathrm{M}_{\odot}\right)$ & $A$ & $n_{0}\left(\mathrm{~cm}^{-3}\right)$ & $B_{0}(\mu \mathrm{G})$ & $\beta$ & $v_{\text {turb }}\left(c_{s}\right)$ & $v_{A}\left(c_{s}\right)$ & $m_{J, B}\left(\mathrm{M}_{\odot}\right)$ & $E_{\text {turb }} / E_{\text {mag }}$ \\
\hline $\mathrm{R} 1$ & 61.1 & 0.9 & 10 & 0.35 & 3.0 & 0.8 & 0.8 & 373.7 & 2.96 \\
$\mathrm{R} 2$ & 40.7 & 0.6 & 90 & 1.06 & 3.0 & 0.8 & 0.8 & 314.1 & 2.90 \\
$\mathrm{R} 3$ & 27.1 & 0.4 & 100 & 1.12 & 3.0 & 0.8 & 0.8 & 326.6 & 2.89 \\
\hline & & & & & & & & & \\
Modelo & $M_{\star}\left(\mathrm{M}_{\odot}\right)$ & $M_{\text {tot }}\left(\mathrm{M}_{\odot}\right)$ & $n_{0}\left(\mathrm{~cm}^{-3}\right)$ & $B_{0}(\mu \mathrm{G})$ & $\beta$ & $v_{\text {turb }}\left(c_{s}\right)$ & $v_{A}\left(c_{s}\right)$ & $m_{J, B}\left(\mathrm{M}_{\odot}\right)$ & $E_{\text {turb }} / E_{\text {mag }}$ \\
\hline & & & & & & & & & \\
$\mathrm{N} 1$ & 61.1 & 69.5 & 10 & 0.35 & 3.0 & 0.8 & 0.8 & 373.7 & 2.96 \\
$\mathrm{~N} 2 \mathrm{a}$ & 40.7 & 49.1 & 10 & 0.35 & 3.0 & 0.8 & 0.8 & 448.1 & 2.96 \\
$\mathrm{~N} 2 \mathrm{~b}$ & 40.7 & 116.4 & 90 & 1.06 & 3.0 & 0.8 & 0.8 & 314.1 & 2.90 \\
$\mathrm{~N} 2 \mathrm{c}$ & 40.7 & 116.4 & 90 & 1.85 & 1.0 & 1.2 & 1.4 & 379.8 & 2.15 \\
$\mathrm{~N} 2 \mathrm{~d}$ & 40.7 & 116.4 & 90 & 3.37 & 0.3 & 1.9 & 2.6 & 639.7 & 1.62 \\
$\mathrm{~N} 2 \mathrm{e}$ & 40.7 & 108.0 & 80 & 1.74 & 1.0 & 1.2 & 1.4 & 389.9 & 2.16 \\
$\mathrm{~N} 3$ & 27.1 & 111.2 & 100 & 1.12 & 3.0 & 0.8 & 0.8 & 326.6 & 2.89 \\
$\mathrm{~N} 4$ & 20.4 & 104.5 & 100 & 1.12 & 3.0 & 0.8 & 0.8 & 339.6 & 2.89 \\
$\mathrm{E} 1$ & 40.7 & 46.6 & 90 & 1.06 & 3.0 & 0.8 & 0.8 & 390.1 & 2.90 \\
\hline
\end{tabular}

$$
t_{d i f f}=\frac{l^{2}}{\eta}
$$

onde a difusividade turbulenta do gás é dada, como vimos no Cap. 4, por (Santos-Lima et al. 2010; Lazarian 2006; 2011; Lazarian et al. 2012b):

$$
\begin{array}{ll}
\eta \sim l_{\text {inj }} v_{\text {turb }} & \text { if } v_{\text {turb }} \geq v_{A}, \\
\eta \sim l_{\text {inj }} v_{\text {turb }}\left(\frac{v_{\text {turb }}}{v_{A}}\right)^{3} & \text { if } v_{\text {turb }}<v_{A},
\end{array}
$$

e $l_{i n j}=L / k_{f}$, e $v_{\text {turb }}=v_{r m s}$. As relações acima indicam que o fator $\left(v_{\text {turb }} / v_{A}\right)^{3}$ é importante somente no regime de turbulência sub-Alfvénica, ou seja, com o número de Mach Alfvénico $M_{A} \leq 1$. Em 5.7 acima, $l$ é uma escala característica menor do que L, 
onde o campo magnético é difundido. Para comparar os modelos, em todas as simulações turbulentas consideramos o mesmo $\eta$. Assim para uma dada escala característica $l$, quando variamos $\beta$ também variamos $v_{\text {turb }}$ de modo a manter $\eta$ constante. Isto assegurou que a turbulência decaísse na mesma escala de tempo em todos os modelos. Na tabela 5.2, o tempo de difusão foi calculado considerando a escala do núcleo da nuvem, i.e., $l \simeq 0.3$ pc.

Tabela 5.2: Escalas de tempo características para os modelos colapsantes.

\begin{tabular}{lccc}
\hline \hline Modelo & $t_{d y n}($ Myrs $)$ & $t_{f f}($ Myrs $)$ & $t_{d i f f}($ Myrs $)$ \\
\hline R1 & 10.6 & 5.7 & 1.4 \\
R2 & 10.6 & 4.4 & 1.4 \\
R3 & 10.6 & 4.5 & 1.4 \\
\hline & & & \\
N1 & 10.6 & 5.7 & 1.4 \\
N2a & 10.6 & 6.7 & 1.4 \\
N2b & 10.6 & 4.4 & 1.4 \\
N2c & 10.6 & 4.4 & 1.4 \\
N2d & 10.6 & 4.4 & 1.4 \\
N2e & 10.6 & 4.5 & 1.4 \\
N3 & 10.6 & 4.5 & 1.4 \\
N4 & 10.6 & 4.6 & 1.4 \\
E1 & 10.6 & 5.6 & 1.4 \\
\hline & & & \\
\hline
\end{tabular}

Para sermos capazes de observar o transporte de fluxo magnético durante a evolução dinâmica das nuvens colapsantes, escolhemos o tempo de difusão turbulento inicial menor do que o tempo de queda livre inicial para todas as simulações. Seguimos a evolução de $B$ e $\rho$ por dez passos de tempo, correspondendo a dez escalas de tempo dinâmicas, ou $\sim 100$ Myrs. Para comparação com os modelos turbulentos também fizemos simulações com condições iniciais similares mas sem injeção de turbulência (estes serão também referidos/mencionados posteriormente como laminar ou modelos não turbulentos). 


\subsection{Resultados das simulações numéricas}

\subsubsection{Modelos com auto-gravidade}

Apresentamos aqui os resultados para nove modelos de nuvens turbulentas onde incluímos os efeitos da auto-gravidade e cujas condições iniciais estão listadas na Tabela 5.1. Todos os modelos exceto N2c, N2d, e N2e, possuem uma velocidade turbulenta igual à velocidade de Alfvén inicial (isto é, são trans-Alfvénicos), enquanto que estes três modelos são subAlfvénicos, isto é, possuem uma velocidade turbulenta menor do que a velocidade de Alfvén. Os modelos fora do equilíbrio N1 e N2a foram iniciados com a mesma densidade do gás $\rho=1.67 \times 10^{-23} \mathrm{~g} / \mathrm{cm}^{3}$, mas com diferentes potenciais gravitacionais (isto é, diferentes valores da massa do potencial estelar $M_{\star}$ ). O modelo $\mathrm{N} 2 \mathrm{~b}$ possui o mesmo potencial estelar inicial que o modelo $\mathrm{N} 2 \mathrm{a}, 40.7 \mathrm{M}_{\odot}$, mas uma densidade de gás maior $\rho=1.503 \times$ $10^{-22} \mathrm{~g} / \mathrm{cm}^{3}$ o que permite comparar os efeitos da auto-gravidade. Modelos N2c e N2d têm a mesma densidade inicial de gás que o modelo anterior, $\mathrm{N} 2 \mathrm{~b}$, mas diferentes valores de $\beta$ o que nos permite comparar os efeitos do campo magnético. O modelo N2e, por sua vez, possui o mesmo $\beta$ inicial que o modelo N2d, porém uma densidade de gás inicial levemente menor $\rho=1.336 \times 10^{-22} \mathrm{~g} / \mathrm{cm}^{3}$, faz com que a turbulência seja mais importante do que no caso anterior. Os modelos N3 e N4 têm o mesmo $\beta=3.0$ inicial e a mesma densidade do gás $\rho=1.67 \times 10^{-22} \mathrm{~g} / \mathrm{cm}^{3}$, mas diferentes potenciais estelares, $27.1 \mathrm{M}_{\odot}$ e $20.4 \mathrm{M}_{\odot}$, respectivamente. Ambos possuem potenciais gravitacionais estelares menores, mas uma maior densidade do gás (e portanto uma auto-gravidade mais significativa) do que os modelos anteriores. Finalmente, o modelo começando em equilíbrio E1 tem condições centrais que são similares às do modelo N2b. As demais condições iniciais são as mesmas para todos os modelos.

Os painéis à esquerda nas Figuras 5.2, 5.4, 5.7, e o painel superior à esquerda da Figura 5.6, mostram mapas de densidade logarítmica do corte central dos modelos simulados após 100 Myrs ou 10 tempos dinâmicos. Os painéis centrais comparam a evolução temporal da razão entre o campo magnético médio e a densidade média dentro de uma esfera (centrada no domínio computacional) de raio $r=0.3 \mathrm{pc}$ que representa o núcleo ou 
caroço da nuvem, $\left(\langle B\rangle_{0.3} /\langle\rho\rangle_{0.3}\right) /(\bar{B} / \bar{\rho})$, para o modelo com turbulência (linha vermelha tracejada) e sem turbulência (linha preta sólida). Os símbolos $<>$ significam médias tomadas dentro do raio da nuvem 0.3 pc. Esta razão é normalizada pelo valor médio $(\bar{B} / \bar{\rho})$ tomado sobre toda a caixa computacional. Para a geometria adotada com um campo magnético inicialmente uniforme, a razão acima é aproximadamente igual à razão fluxo magnético-massa dentro da região considerada.

Os painéis à direita destas figuras $(5.2,5.4,5.7)$ e o painel superior à direita da Figura 5.6 apresentam perfis da razão entre o campo magnético médio e a densidade média como função da distância radial $r$ do centro da nuvem para estes mesmos modelos em $t=100$ Myrs.

Na Figura 5.2, notamos que após uma rápida diminuição inicial causada pela relaxação do sistema, a razão campo magnético-densidade dentro do núcleo da nuvem (painéis ao centro) permanece aproximadamente constante no caso laminar (isto é, sem turbulência), enquanto nos casos com turbulência existe uma clara diminuição desta ${ }^{2}$. Este resultado indica que existe transporte de fluxo magnético a partir das regiões centrais mais massivas e densas para as regiões de menor densidade fora do núcleo das nuvens. Na Figura 5.2, este efeito é particularmente mais pronunciado no modelo N2b (painel inferior) possui uma densidade inicial de gás muito maior do que os outros modelos da Figura, e portanto, está sob a influência de uma auto-gravidade maior. Comparando os modelos N1 e N2a que têm a mesma densidade inicial de gás, notamos que aquele com maior potencial estelar (modelo N1 no painel superior) apresenta um maior desacoplamento entre o fluxo magnético e a densidade. Consistentemente, os painéis à direita da Figura 5.2 mostram que o modelo turbulento com maior densidade inicial (para o qual os efeitos da autogravidade são mais importantes, i.e., o modelo N2b), tem uma maior diminuição no perfil radial da razão campo magnético-densidade na região do núcleo da nuvem (acompanhada por um grande aumento desta nas regiões do envelope da nuvem) confirmando portanto, a tendência observada nos painéis centrais.

\footnotetext{
${ }^{2}$ Notamos que as oscilações observadas nestes gráficos (que são levemente mais fortes nos modelos laminares) são oscilações acústicas da nuvem devido ao fato de que o tempo de virialização destes sistemas é maior do que o período simulado.
} 

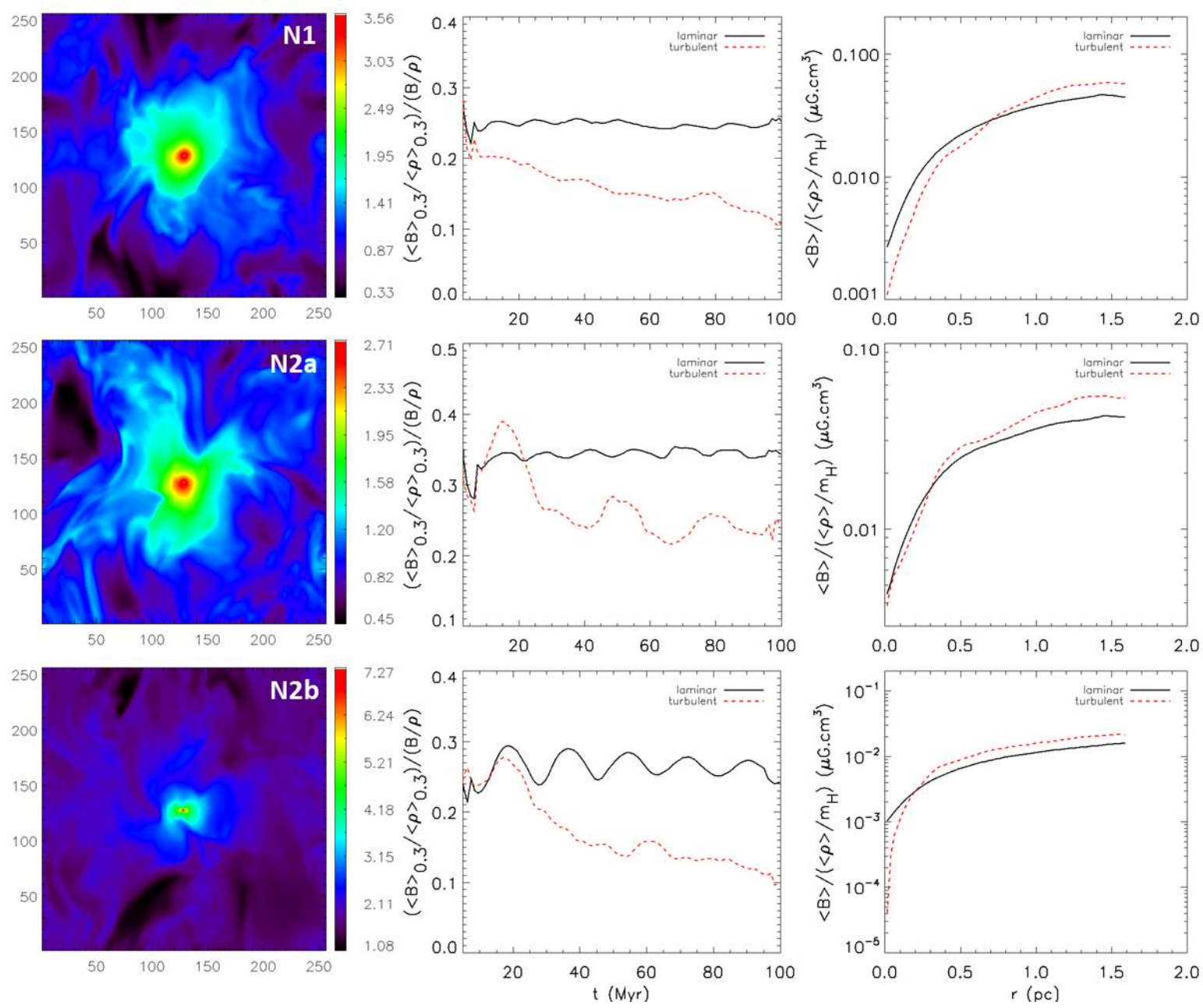

Figura 5.2: Modelo N1 com $M_{\text {pot }}=61.1 \mathrm{M}_{\odot}$ (em cima), modelos N2a (centro) e N2b (embaixo) com $M_{p o t}=40.7 \mathrm{M}_{\odot} \cdot$ Modelos superior e central possuem densidades iniciais $n_{0}=10 \mathrm{~cm}^{-3}$, e o modelo inferior, N2b, tem $n_{0}=90 \mathrm{~cm}^{-3}$. Todos os modelos possuem $\beta=3.0$. Painéis à esquerda mostram mapas logarítmicos da densidade de um corte central dos modelos turbulentos em $t=100$ Myrs. Os painéis centrais mostram a evolução temporal da razão campo magnético médio-densidade média no núcleo da nuvem de raio $r_{c}=0.3$ pc normalizado pelo valor médio sobre toda a nuvem, $\left(\langle B\rangle_{0.3} /\langle\rho\rangle_{0.3}\right) /(\bar{B} / \bar{\rho})$, para os modelos turbulento (linhas vermelhas tracejadas) e laminar (linhas pretas sólidas). Painéis à direita apresentam o perfil radial da razão entre campo magnético médio e a densidade média em $t=100$ Myrs $\langle B\rangle /\langle\rho\rangle$ para estes modelos. 

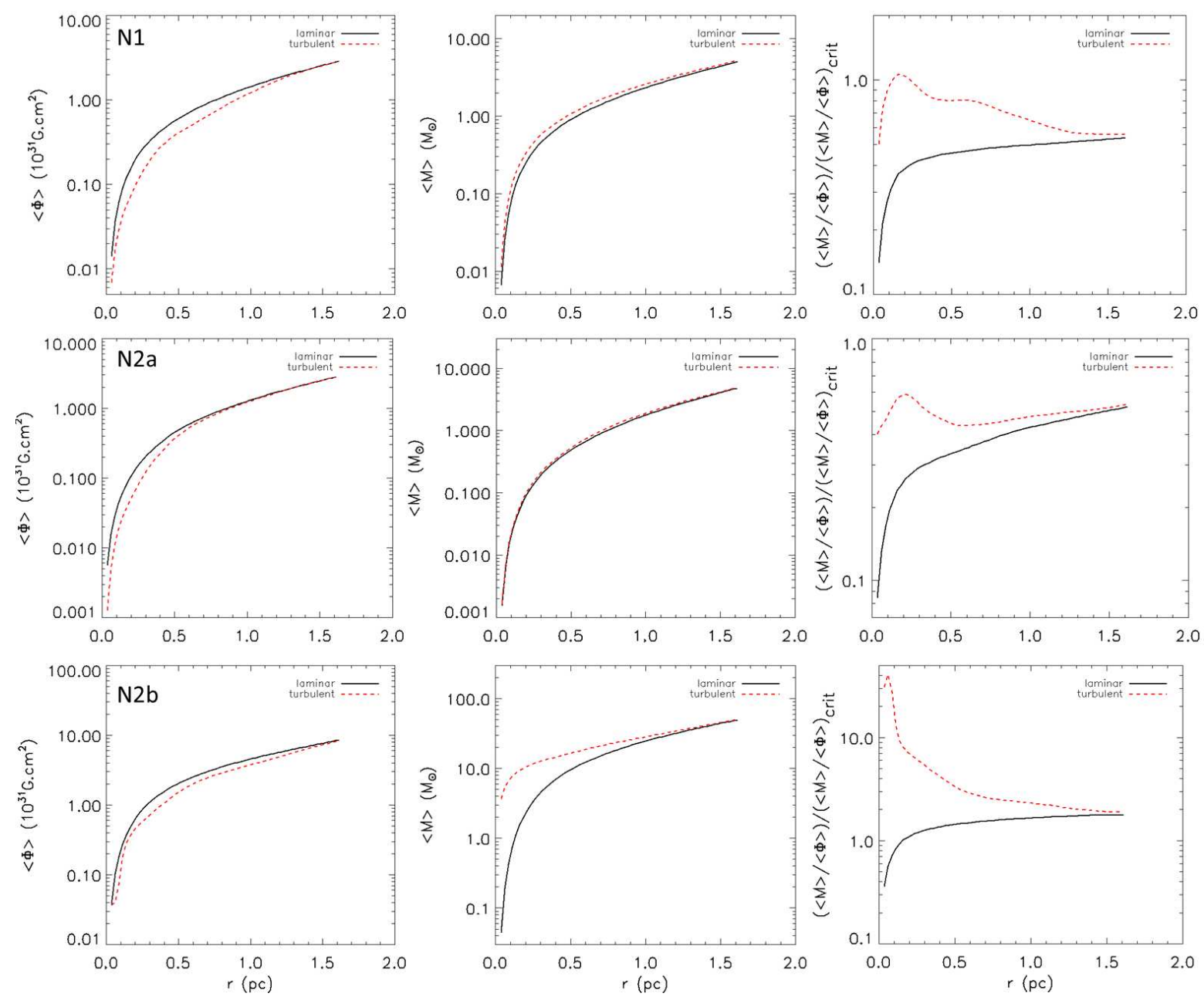

Figura 5.3: Perfis radiais em $t=100$ Myr para o fluxo magnético $\Phi$ (painéis à esquerda), a massa gasosa $M$ (painéis centrais), e para a razão massa-fluxo magnético normalizada pelo valor crítico $(M / \Phi) /(M / \Phi)_{\text {crit }}$ (painéis à direita) para os modelos da Figura 5.2. Painéis superiores: modelo N1 $\left(n_{0}=10 \mathrm{~cm}^{-3}\right.$, e $\left.M_{\star}=61.1 \mathrm{M}_{\odot}\right)$; painéis centrais: modelo $\mathrm{N} 2 \mathrm{a}\left(n_{0}=90 \mathrm{~cm}^{-3}\right.$, e $\left.M_{\text {pot }}=40.7 \mathrm{M}_{\odot}\right)$; painéis inferiores: modelo $\mathrm{N} 2 \mathrm{~b}\left(n_{0}=10 \mathrm{~cm}^{-3}\right)$. Linhas vermelhas tracejadas são para os modelos turbulentos e linhas contínuas pretas são para os modelos laminares.

Para verificar se uma nuvem inicialmente subcrítica desenvolveu caroços supercríticos após a ação do transporte de fluxo magnético por reconexão turbulenta, as Figuras 5.3, 5.5 e 5.8, e os painéis inferiores da Figura 5.6 comparam os perfis radiais integrados do fluxo magnético (painéis à esquerda), da massa do gás (painéis centrais), e da razão massa-fluxo 
magnético normalizada pelo valor crítico (direita) em $t=100 \mathrm{Myr}$ para os modelos com e sem turbulência ${ }^{3}$. Por exemplo, na Figura 5.3, vemos que os modelos N1 e N2b da Figura 5.2 desenvolvem caroços supercríticos, enquanto que o modelo N2a permanece subcrítico em toda sua extensão.
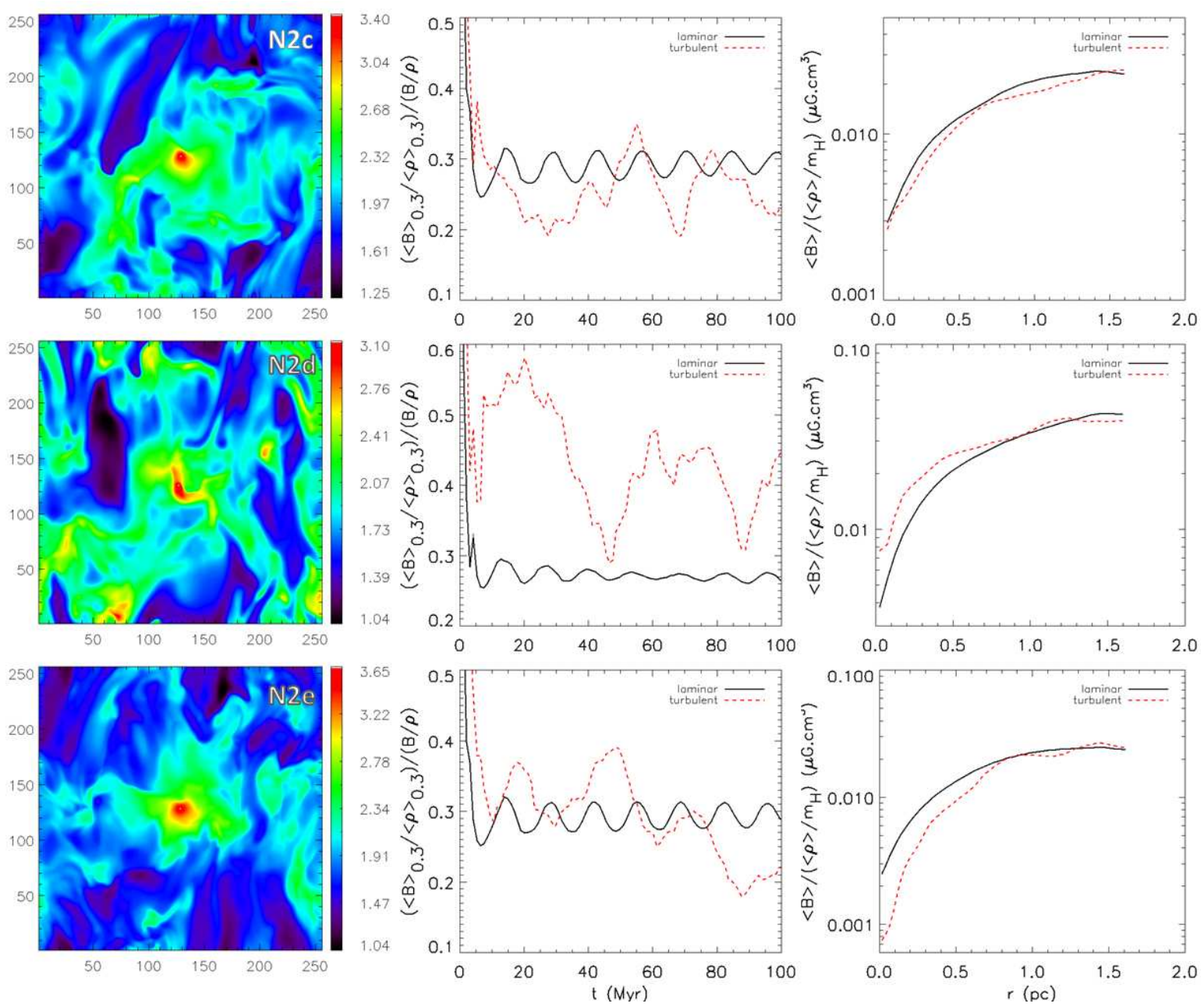

Figura 5.4: O mesmo que na Figura 5.2 para os modelos N2c, N2d e N2e. Painéis superior e inferior: modelos N2c e N2e com $\beta=1.0$ Centro: modelo N2d com $\beta=0.3$. Modelos superior e central possuem densidades $n_{0}=90 \mathrm{~cm}^{-3}$, e o modelo inferior possui $n_{0}=80$ $\mathrm{cm}^{-3}$. Todos os modelos têm $M_{p o t}=40.7 \mathrm{M}_{\odot}$

As Figuras 5.4 e 5.5 mostram os mapas logarítmicos de densidade e perfis similares

\footnotetext{
${ }^{3} \mathrm{O}$ valor crítico para a razão massa-fluxo magnético calculado para uma nuvem esférica é dado por: $(\pi \sqrt{(18 \mathrm{G}) / 5})^{-1}$
} 

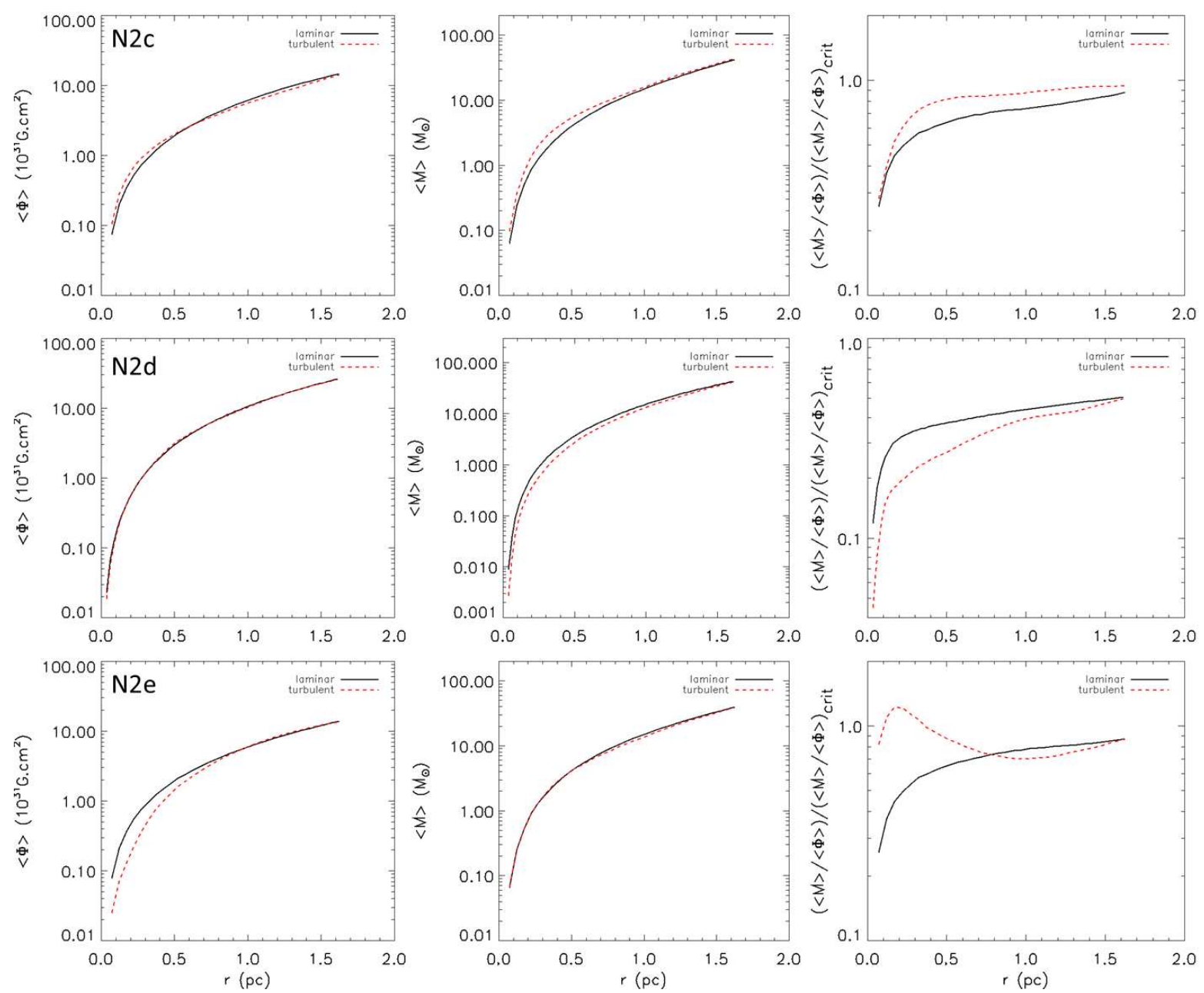

Figura 5.5: O mesmo que na Figura 5.3 para os modelos N2c, N2d e N2e. Painéis superiores: modelo N2c $\left(n_{0}=90 \mathrm{~cm}^{-3}\right.$, e $\left.\beta=1.0\right)$; painéis centrais: modelo N2d $\left(n_{0}=90\right.$ $\mathrm{cm}^{-3}$, e $\left.\beta=0.3\right)$; painéis inferiores: modelo N2e $\left(n_{0}=80 \mathrm{~cm}^{-3}\right.$, e $\left.\beta=1.0\right)$.

àqueles da Figura 5.2 para os modelos com turbulência sub-Alfvénica N2c, N2d, e N2e. Os modelos N2c e N2d têm a mesma densidade de gás inicial e massa estelar que o modelo $\mathrm{N} 2 \mathrm{~b}$, mas menor $\beta$ inicial e menor razão entre a velocidade turbulenta e a velocidade de Alfvén. Isto naturalmente faz com que o desacoplamento entre o fluxo magnético e o gás das regiões mais densas pela reconexão turbulenta seja mais difícil. Ambos os modelos N2c e N2d, para os quais $\beta=1.0$, e 0.3, respectivamente, não apresentam transporte de fluxo magnético para fora significativo quando comparados ao modelo N2b da Figura 5.2 (para o qual $\beta=3.0$ ), ou à suas contrapartidas laminares. Contudo, quando a densidade 
inicial do modelo N2c é diminuída de 90 para $80 \mathrm{~cm}^{-3}$ (mantendo $\beta=1.0$ ), como no modelo N2e, o transporte de fluxo magnético pela turbulência é ampliado (embora seja ainda menor que no modelo N2b) e a nuvem inicialmente subcrítica desenvolve um glóbulo aproximadamente crítico após 100 Myrs (veja Figura 5.5). Portanto, este resultado indica que o aumento da densidade do gás e da gravidade total nem sempre irá resultar em um aumento do transporte de fluxo pela turbulência, como vemos nos modelos da Figura 5.2. Quando a turbulência é sub-Alfvénica (e portanto o campo magnético é forte para a turbulência aplicada), um potencial gravitacional menor irá atrasar o colapso dando assim tempo para que a difusão por reconexão turbulenta transporte parte do fluxo magnético para as regiões externas. Isto explica porquê a diminuição da densidade de $n_{0}=90 \mathrm{~cm}^{-3}$ no modelo N2c para $n_{0}=80 \mathrm{~cm}^{-3}$ no modelo N2e resulta na formação de um glóbulo aproximadamente crítico neste último.
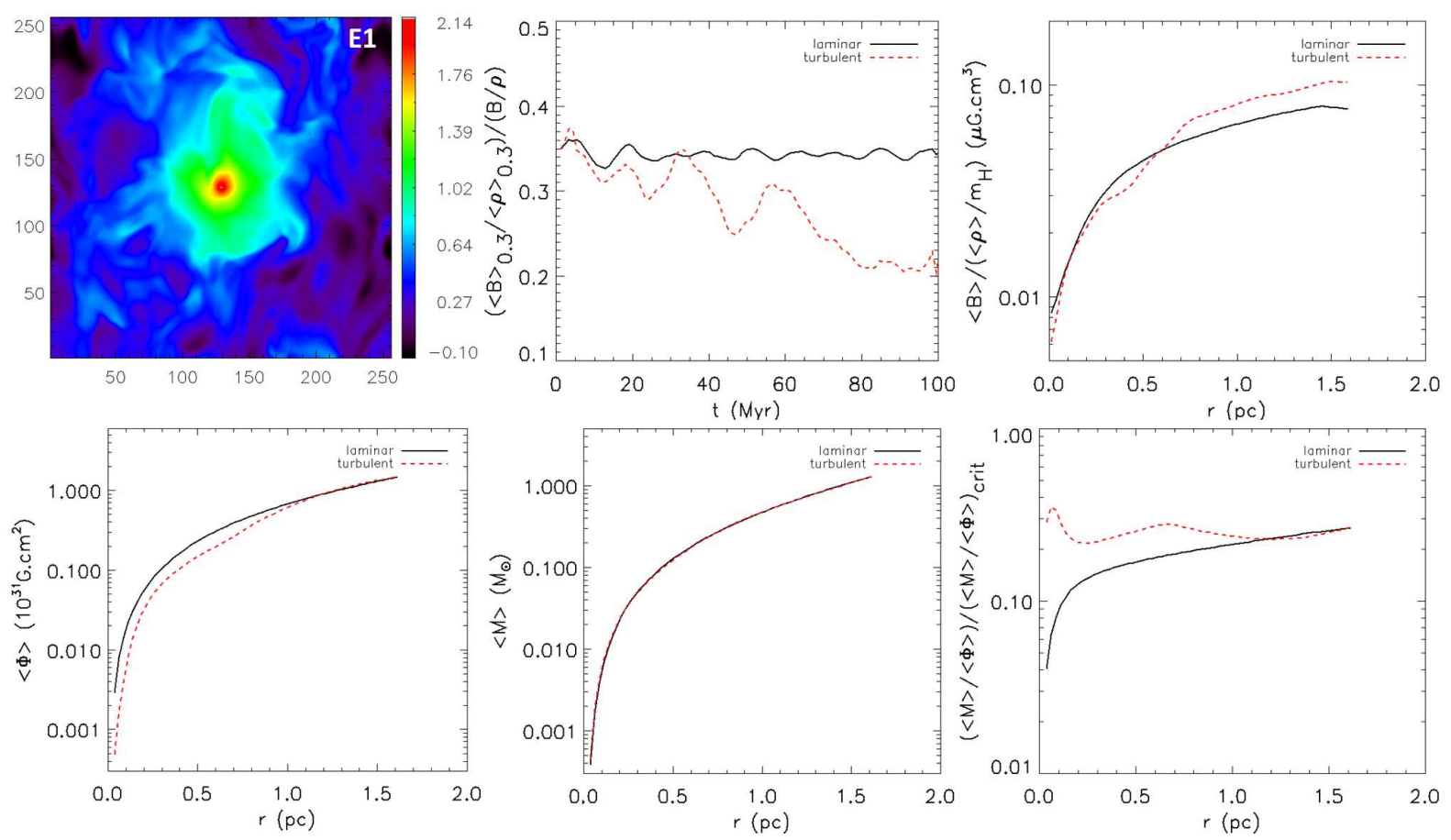

Figura 5.6: Modelo em equilíbrio E1 com $M_{\text {pot }}=61.1 \mathrm{M}_{\odot}$. Este modelo possui densidade inicial central $n_{0}=90 \mathrm{~cm}^{-3}$ e $\beta=3.0$. Em cima: o mesmo que na Figura 5.2. Embaixo: o mesmo que na Figura 5.3.

A Figura 5.6 apresenta o mapa de densidade logarítmica e os perfis radiais para o 
modelo E1. Este modelo que se inicia em equilíbrio magneto-hidrostático possui o mesmo $\beta$ e densidade $\rho_{0}$ iniciais em sua região central que o modelo fora do equilíbrio N2b, mas a condição de equilíbrio faz com que seu perfil de densidade seja estratificado e assim a massa dentro da nuvem é menor do que no modelo N2b (veja Tabela 5.1). Como no modelo turbulento N2b, E1 também sofre transporte de fluxo magnético, conforme indicado pela diminuição da razão campo magnético-densidade na região central da nuvem (painel superior ao centro). Contudo, a massa total muito menor de E1 impede ao núcleo de colapsar e tornar-se um glóbulo supercrítico, como indicado pelo diagrama massa-fluxo magnético da Figura 5.6 (painel inferior à direita, linha vermelha tracejada).
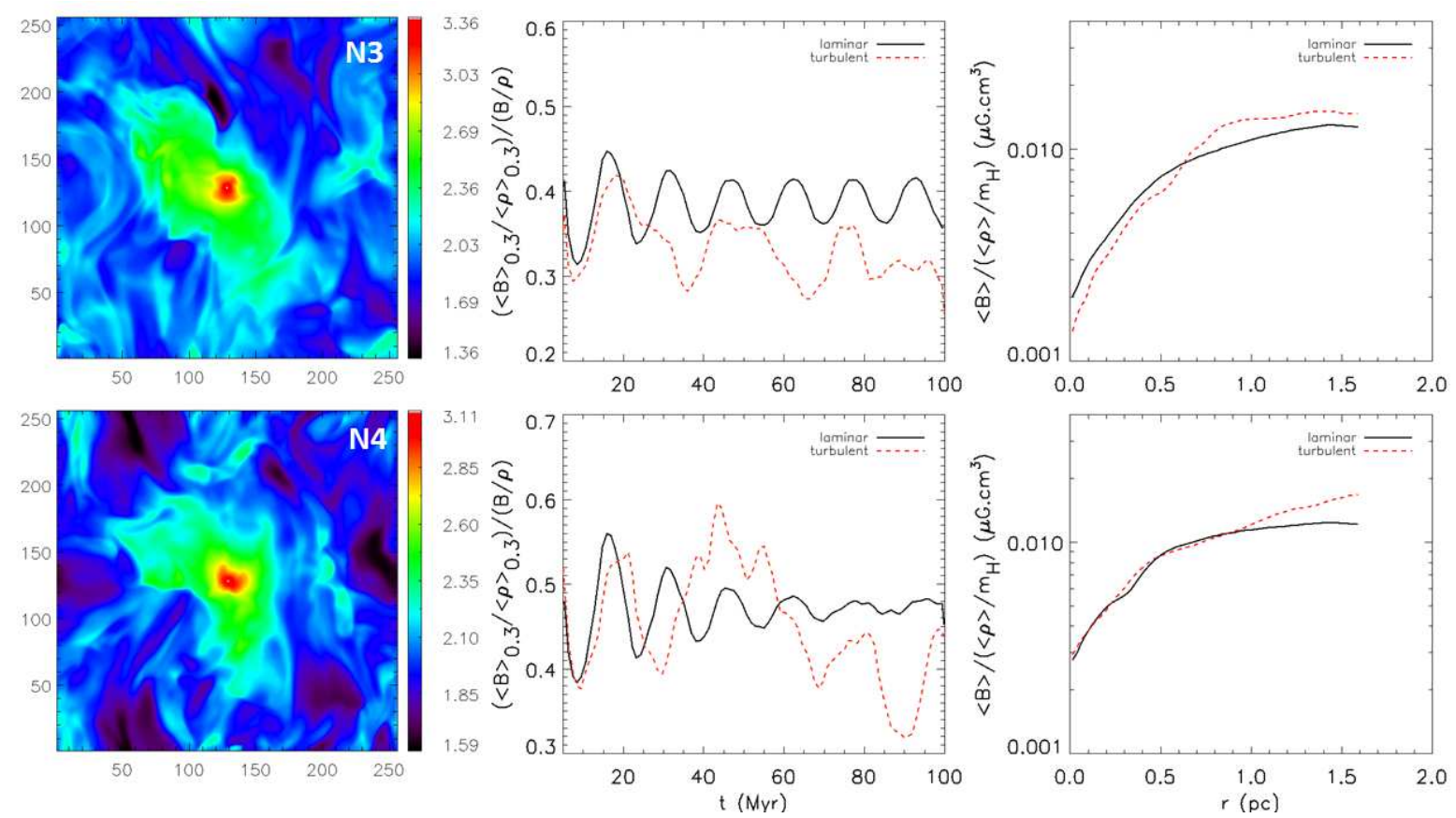

Figura 5.7: O mesmo que na Figura 5.2 para os modelos fora do equilíbrio N3 e N4. Em cima: modelo $\mathrm{N} 3$ com $M_{\text {pot }}=27.1 \mathrm{M}_{\odot}$. Embaixo: modelo N4 com $M_{\text {pot }}=20.4 \mathrm{M}_{\odot}$. Ambos os modelos possuem densidades iniciais $n_{0}=100 \mathrm{~cm}^{-3}$ e $\beta=3.0$.

As Figuras 5.7 e 5.8 apresentam os resultados para os modelos N3 e N4 que possuem o mesmo $\beta=3.0$ inicial e densidade do gás $n_{0}=100 \mathrm{~cm}^{-3}$ (os quais são comparáveis com o modelo N2b para o qual a densidade inicial de gás é ligeiramente menor), mas potenciais gravitacionais estelares, $27.1 \mathrm{M}_{\odot}$ e $20.4 \mathrm{M}_{\odot}$, respectivamente (os quais são menores do que 

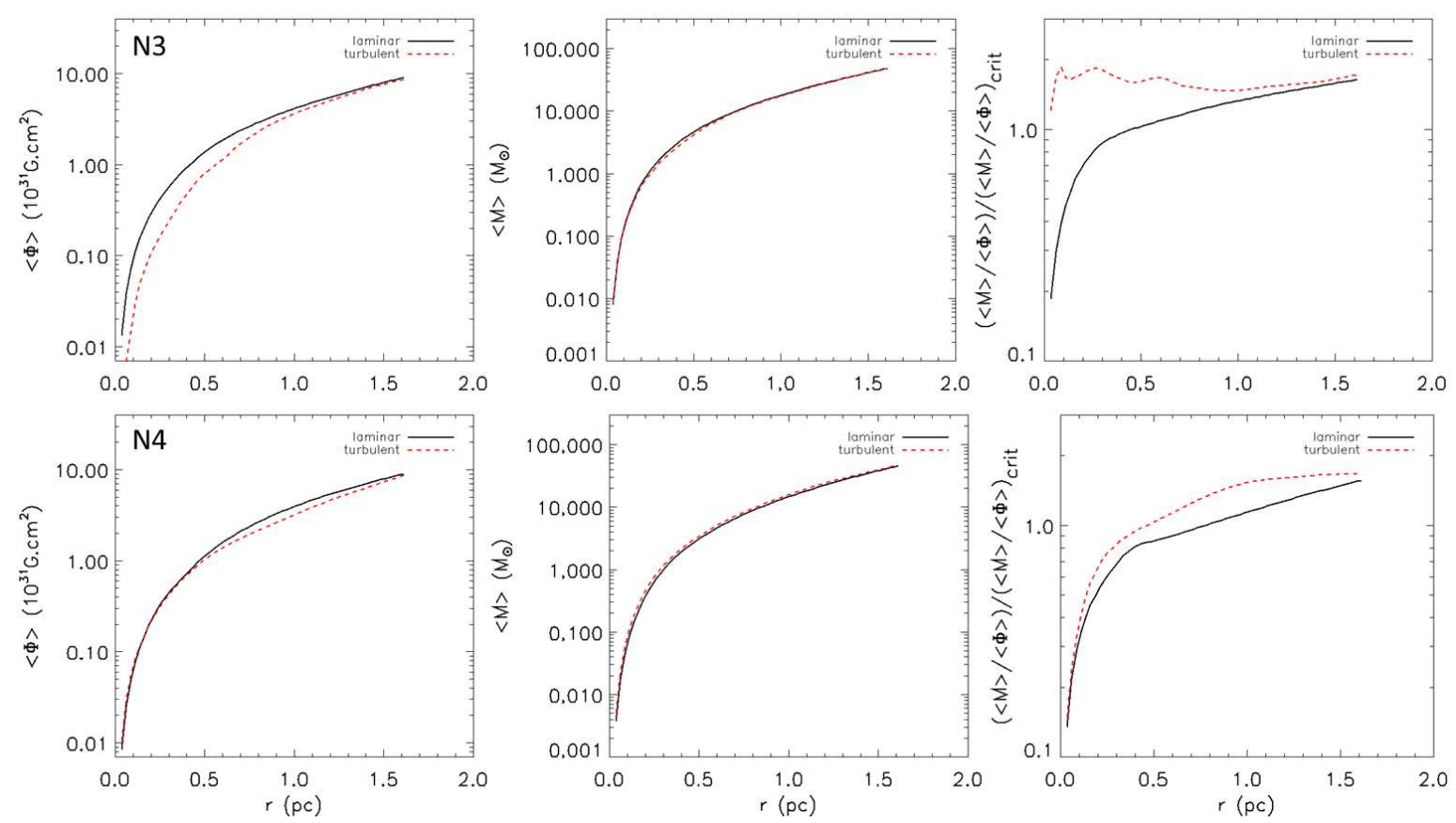

Figura 5.8: O mesmo que na Figura 5.3 para os modelos N3 e N4 da Figura 5.7. Painéis superiores: modelo N3 $\left(n_{0}=100 \mathrm{~cm}^{-3}\right.$, e $\left.M_{\star}=27.1 \mathrm{M}_{\odot}\right)$; painéis inferiores: modelo N4 $\left(n_{0}=100 \mathrm{~cm}^{-3}\right.$, e $\left.M_{\star}=20.4 \mathrm{M}_{\odot}\right)$.

no modelo N2b).

Em ambos os modelos turbulentos notamos uma diminuição menor na razão campo magnético-densidade no núcleo da nuvem ao longo do tempo quando comparados ao modelo N2b (veja painéis centrais da Figura 5.7), especialmente para o modelo com menor potencial estelar (N4). A inspeção do perfil radial da razão massa-fluxo magnético em $t=100$ Myr para este modelo na Figura 5.8 mostra que de fato não existe transporte de fluxo magnético neste caso. Isto ocorre por causa da combinação de dois efeitos. O maior campo magnético do modelo N4 relativo ao modelo N2b (veja Tabela 5.1) faz com que seja mais difícil para a turbulência desacoplar o fluxo magnético do material mais denso. Ao mesmo tempo com um menor potencial gravitacional estelar a queda de gás para o centro no modelo N4 é mais lenta (veja Tabela 5.2), e a turbulência torna-se mais eficaz para espalhar o gás agindo contra a gravidade (painel inferior à esquerda da Figura 5.8). O modelo N3 por outro lado, embora tenha a mesma força de campo magnético 
que no modelo N4, tem um potencial estelar grande o bastante para reter a matéria do núcleo da nuvem enquanto a turbulência desacopla o fluxo magnético transportando-o para as regiões menos densas do envelope, permitindo assim a formação de um glóbulo supercrítico (como vemos no painel superior da Figura 5.8).

\subsubsection{Os efeitos da resolução nos resultados}

Como mencionado, todas as simulações numéricas apresentadas acima foram realizadas com uma resolução de $256^{3}$. Para assegurar-nos de que os resultados não foram afetados por efeitos numéricos relevantes, também realizamos simulações de um dos modelos (modelo N2e, Tabela 5.1) com resolução maior e menor que 256 . A Fig. 5.9 apresenta diagramas da razão campo magnético-densidade tal como na Figura 5.4 (painel central) para este modelo com resoluções $128^{3}, 256^{3}$, e $512^{3}$. Não se observa diferenças significativas entre eles, particularmente entre os modelos com resolução $256^{3}$ e $512^{3}$, caracterizando assim a convergência dos resultados para os primeiros. Assim, isso sugere que os resultados apresentados para os modelos com resolução $256^{3}$ são robustos.

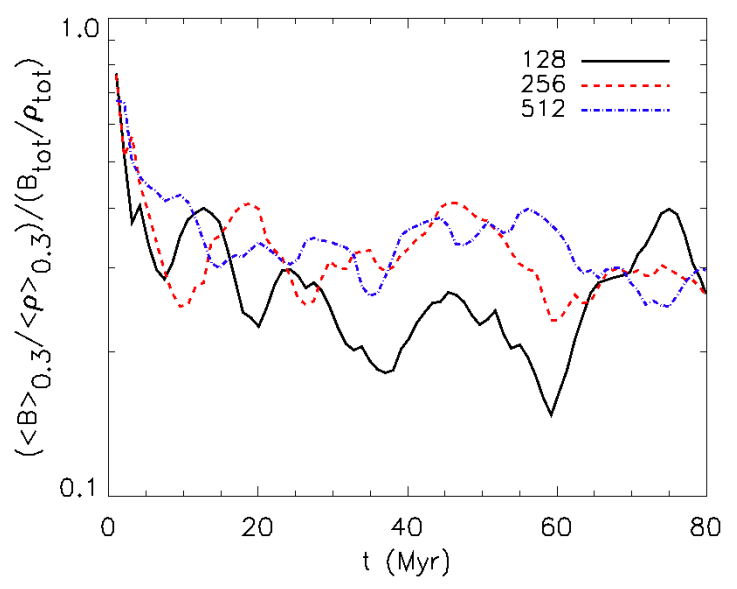

Figura 5.9: Comparação entre resoluções diferentes para o modelo N2e (Figuras 5.4 e 5.5 e Tabela 5.1). 


\subsubsection{Modelos sem auto-gravidade}

Não discutimos ainda os efeitos específicos que a inclusão da auto-gravidade produz sobre o colapso de um núcleo turbulento. Para tratar deste assunto devemos comparar os modelos auto-gravitantes analisados na seção anterior com suas contra-partida sem autogravidade. Estes modelos denominados " R"' estão também listados na Tabela 5.1. O modelo R1 tem condições iniciais similares à do modelo auto-gravitante N1 da Tabela 5.1, com um parâmetro de potencial gravitacional $A=0.9$, o qual é equivalente à massa estelar $61.7 \mathrm{M}_{\odot}$. Os modelos R2 e R3, por sua vez, tem condições iniciais similares aos modelos auto-gravitantes N2b e N3 da Tabela 5.1, com $M_{\star}=40.7 \mathrm{M}_{\odot} \rightarrow A=0.6$, e $M_{\star}=27.1 \mathrm{M}_{\odot} \rightarrow A=0.4$, respectivamente.

Os mapas de densidade logarítmica em 100 Myrs bem como os perfis radiais e a evolução temporal relativos a estes modelos são encontrados nas Figuras 5.10 e 5.11.

A comparação dos modelos sem auto-gravidade R2 e R3 com os modelos auto-gravitantes N2b e N3, respectivamente (painéis inferiores nas Figuras 5.2 e 5.3 e painéis superiores nas Figuras 5.7 e 5.8) revelam a importância da inclusão de auto-gravidade, particularmente no modelo R2 que não desenvolve um núcleo supercrítico ao contrário de sua contra-partida auto-gravitante (modelo N2b). Por outro lado, o modelo R1 (painéis superiores nas Figuras 5.10 e 5.11) não é muito afetado pela eliminação da auto-gravidade como podemos observar comparando-o com o modelo auto-gravitante N1 (painéis superiores das Figuras 5.2 e 5.3). Neste caso o potencial gravitacional externo devido às estrelas embebidas é tão alto que domina o colapso da nuvem fazendo com que a auto-gravidade seja não desprezível somente em um raio muito pequeno próximo do centro.

O modelo R1 também é comparável com o modelo D2 de Santos-Lima et al. (2010). Ambos possuem as mesmas condições iniciais e são sem auto-gravidade, mas diferem na geometria, as nuvens em Santos-Lima et al. têm um potencial gravitacional inicial cilíndrico ao invés de esférico. A presença de um campo gravitacional esférico produz uma eficiência menor no transporte de fluxo magnético, como podemos notar quando comparamos o diagrama superior central da Figura $5.2 \mathrm{com}$ o diagrama superior direito da Figura 11 de Santos-Lima et al. (2010). Isto ocorre pois no caso de um potencial 
esférico toda a matéria é puxada para um único ponto enquanto que no caso do potencial cilíndrico a gravidade puxa o material colapsante para o eixo central ao longo de todo o cilindro o que é mais efetivo para auxiliar o desacoplamento entre o gás colapsante e o fluxo magnético pela difusão por reconexão turbulenta.

\subsection{Discussões}

Apresentamos neste capítulo simulações 3D MHD da evolução de nuvens auto-gravitantes com um potencial gravitacional central com simetria esférica imersas em um campo magnético inicialmente uniforme. Para comparação consideramos também alguns modelos sem auto-gravidade. As simulações foram iniciadas com a nuvem em equilíbrio magneto-hidrostático (veja modelo E1 da Tabela 5.1) ou já fora deste (todos os outros modelos da Tabela 5.1). Injetamos turbulência não-helicoidal ${ }^{4}$ em cada sistema com uma velocidade rms $\left(v_{r m s}\right)$ comparável à velocidade de Alfvén inicial e à velocidade isotérmica do som (veja a Tabela 5.1) e então deixamos o sistema evoluir. Todos os modelos testados possuem uma razão entre a energia turbulenta e a energia magnética $>1$, o que é compatível com estimativas de observações do meio interestelar frio neutro (Heiles \& Troland 2005). Impusemos a todos os sistemas o mesmo tempo de decaimento turbulento (eqs. 5.7) para compararmos os efeitos da difusão por reconexão turbulenta entre eles. Também para comparação, evoluímos o mesmo grupo de modelos sem a injeção de turbulência.

A maioria dos modelos turbulentos investigados aqui mostra evidências de transporte de fluxo magnético, isto é, um desacoplamento do campo magnético das regiões internas mais densas da nuvem devido à presença de turbulência MHD. As exceções são o modelo R3 sem auto-gravidade (como demonstrado pelas Figuras 5.10 e 5.11) e os modelos autogravitantes N2c, N2d, e N4 (veja as Figuras 5.4 e 5.5, e 5.7 e 5.8, respectivamente).

\footnotetext{
${ }^{4}$ Turbulência não-helicoidal é a turbulência em que a helicidade cinética $(\mathbf{v} \cdot \nabla \times \mathbf{v})$ é diferente de zero, ou seja, $\nabla \times v$ é diferente de zero (Haugen et al. 2004; Polifke \& Shtilman 1989), esta é importante para estudos de dínamo e a indução do efeito alfa nestes.
} 

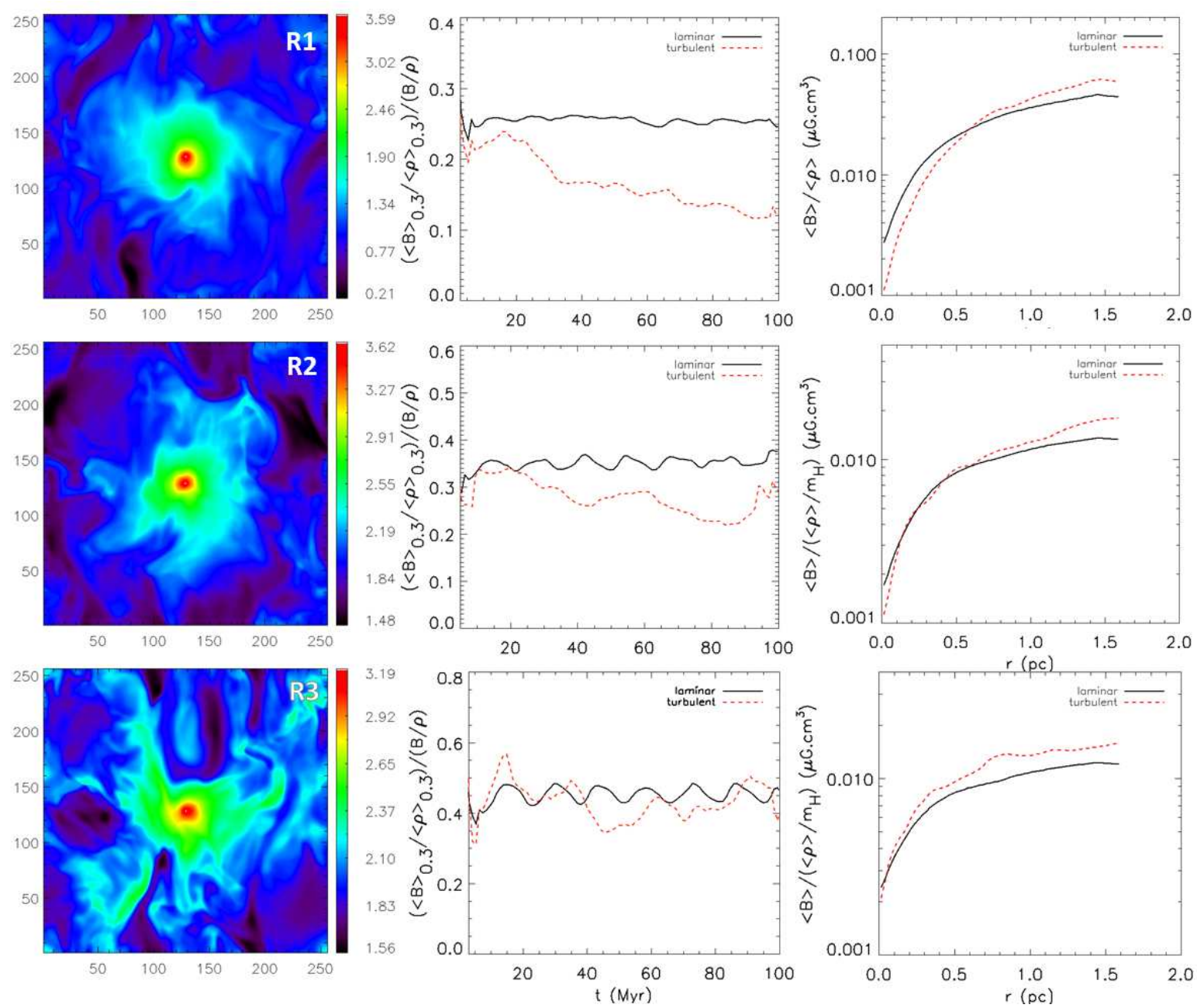

Figura 5.10: Modelo R1 com $A=0.9\left(M_{\text {pot }}=61.1 \mathrm{M}_{\odot}\right)$ (em cima), modelo R2 (centro) $\operatorname{com} A=0.6\left(M_{\text {pot }}=40.7 \mathrm{M}_{\odot}\right)$, e modelo R3 (embaixo) com $A=0.4\left(M_{\text {pot }}=27.1 \mathrm{M}_{\odot}\right)$. Os modelos tem densidades iniciais $\rho_{0}=1.0$, e $\beta=3$.0. Painéis à esquerda mostram mapas de densidade logarítmica do corte central no plano xz dos modelos de nuvens turbulentas em $t=100$ Myrs. Os painéis centrais mostram a evolução temporal da razão entre o campo magnético médio e a densidade na região do núcleo da nuvem de raio $r_{c}=0.3 \mathrm{pc}$ normalizada pelo valor médio sobre toda a nuvem, $\left(\langle B\rangle_{0.3} /\langle\rho\rangle_{0.3}\right) /(\bar{B} / \bar{\rho})$, para os modelos turbulento (linhas tracejadas vermelhas) e laminar (linhas contínuas pretas). Os painéis à direita mostram o perfil radial médio da razão campo magnético-densidade em $t=100$ Myrs $\langle B\rangle /\langle\rho\rangle$ para estes modelos. 

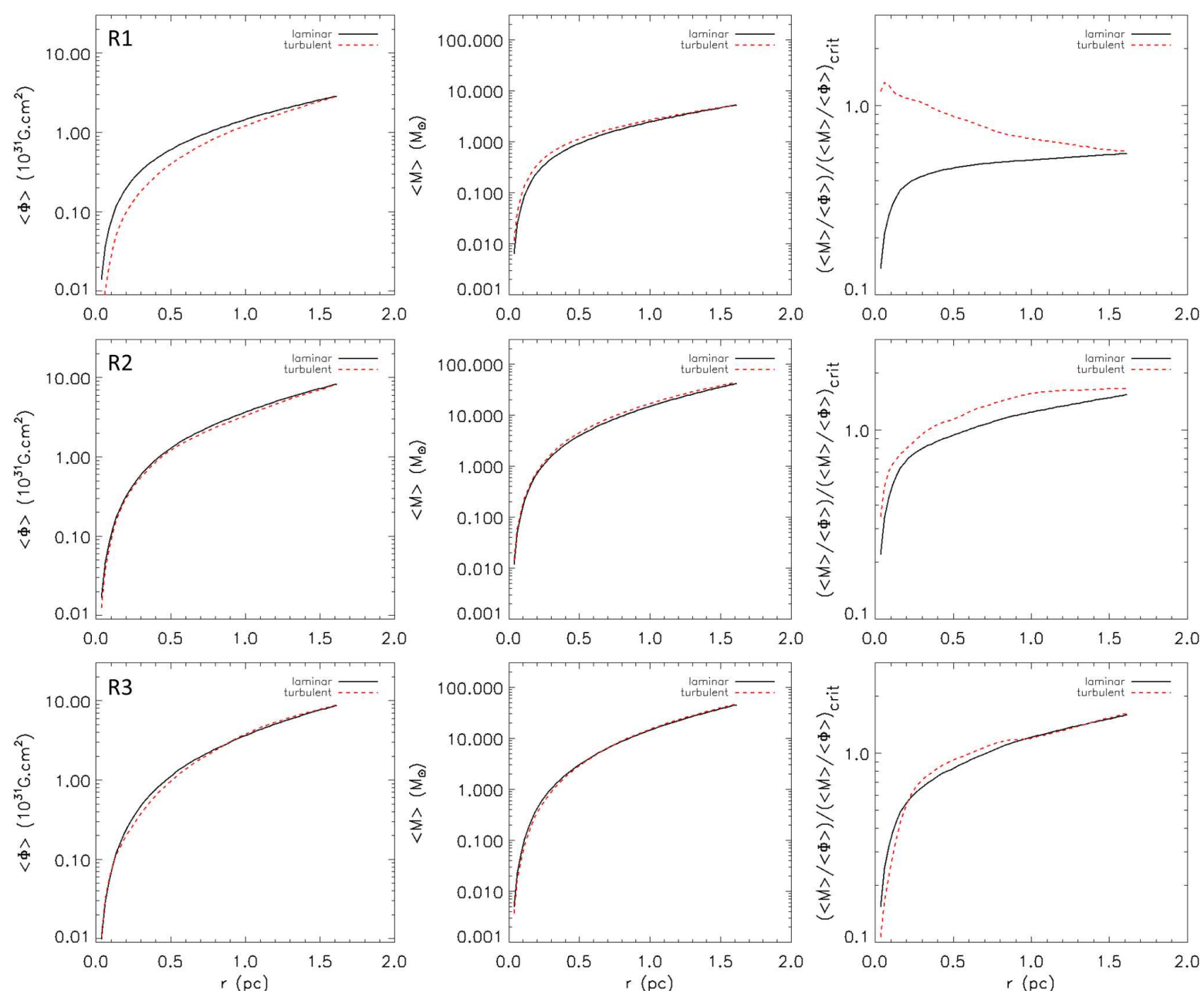

Figura 5.11: Perfis radiais em $t=100$ Myrs para o fluxo magnético $\Phi$ (painéis à esquerda), a massa gasosa $M$ (painéis centrais), e a razão massa-fluxo magnético normalizada pelo valor crítico $(M / \Phi) /(M / \Phi)_{\text {crit }}$ (painéis à direita) para os modelos da Figura 5.11. Painéis superiores: modelo R1 $\left(A=0.9\right.$, ou seja, $\left.M_{\star}=61.1 \mathrm{M}_{\odot}\right)$; painéis centrais: modelo R2 $\left(A=0.6\right.$, ou $\left.M_{\star}=41.7 \mathrm{M}_{\odot}\right) ;$ painéis inferiores: modelo $\mathrm{R} 3\left(A=0.4\right.$, ou $\left.M_{\star}=27.1 \mathrm{M}_{\odot}\right)$. Linhas tracejadas vermelhas são para os modelos turbulentos e linhas contínuas pretas para os laminares.

\subsubsection{Comparação com os resultados de Santos-Lima et al. (2010)}

Estudos numéricos anteriores de sistemas de nuvens cilíndricas realizados por SantosLima et al.(2010; veja também Lazarian 2011; de Gouveia Dal Pino et al. 2011) já mostraram a importância dos efeitos da reconexão magnética turbulenta para remover o 
excesso de fluxo magnético de sistemas colapsantes, como sugerido por Lazarian (2005). O presente estudo, que considera nuvens auto-gravitantes esféricas mais realistas, confirma os resultados da análise anterior, ou seja, mostra que a presença de turbulência é capaz de remover fluxo magnético de regiões mais densas centrais para as bordas da nuvem, e portanto, facilita o colapso gravitacional na maioria dos modelos testados, sem considerar os efeitos da difusão ambipolar. Isto é assegurado pela medida da razão fluxo magnéticomassa na região do núcleo das nuvens simuladas, o qual é quantificado em nosso estudo pela razão entre campo magnético médio e a densidade média dentro destas (veja os diagramas centrais das Figuras 5.2, 5.4, 5.6-em cima, 5.7, e 5.10). Esta razão diminui com o tempo na maioria dos modelos turbulentos, enquanto permanece constante, em média, em suas contra-partidas não turbulentas.

Como em Santos-Lima et al. (2010), também encontramos que um aumento no potencial gravitacional estelar (veja e.g., os modelos N1 e N2a da Figura 5.2), bem como uma diminuição na força do campo magnético inicial (ou um aumento em $\beta$; veja modelos N2b da Figura 5.2 e N2c e N2d na Figura 5.4) favorece o transporte de fluxo magnético por reconexão magnética turbulenta e seu desacoplamento do gás denso colapsante.

Nos casos em que a turbulência é sub-Alfvénica (i.e., $v_{t u r b} / v_{A}<1$, como nos modelos N2c, N2d, e N2e), o transporte de fluxo pela reconexão turbulenta é mais difícil, como deveríamos esperar, já que a turbulência nas grandes escalas é fraca. De fato, para uma nuvem com um campo magnético inicial muito grande, turbulência sub-Alfvénica não será capaz de transportar o fluxo magnético para fora e o núcleo da nuvem falhará em evoluir para um núcleo supercrítico, isto é, um núcleo com razão massa-fluxo magnético acima do valor crítico, o que é necessário para a gravidade se sobrepor às forças magnéticas (veja os modelos N2c e N2d nas Figuras 5.4 e 5.5 para os quais $\beta=0.3$ e $v_{\text {turb }} / v_{A}=0.7$ e 0.9 , respectivamente).

No entanto, até mesmo regimes de turbulência sub-Alfvénica podem permitir a formação de núcleos críticos. Este foi o caso do modelo N2e, que possui as mesmas condições iniciais do modelo N2c, porém, um potencial gravitacional total (gás + estrelas) menor. Este provocou um atraso no colapso de gás, o qual deu tempo para a reconexão turbulenta sub-Alfvénica agir, pois esta se torna mais forte nas menores escalas (Lazarian 2006, 2011), 
e transportar para fora parte do fluxo magnético e permitindo a formação de um núcleo crítico (veja Figura 5.5).

Este resultado pode ser compreendido em termos do coeficiente de difusão por reconexão em regimes sub-Alfvénicos (eq. 4.17, Cap. 4). Embora fraca nas grandes escalas, à medida que a turbulência cascateia a força das interações aumenta e em uma escala $l \simeq l_{\text {inj }}\left(v_{\text {turb }} / v_{A}\right)^{2}$ torna-se mais forte e, portanto, mais eficiente para ajudar a transportar o fluxo magnético. Como vimos no Cap.4, a difusividade neste regime é dada aproximadamente por $l_{i n j} v_{t u r b}\left(v_{t u r b} / v_{A}\right)^{3}$ (eq. 4.17). Logo é menor do que a difusividade turbulenta hidrodinâmica por um fator $\left(v_{\text {turb }} / v_{A}\right)^{3}$ (ver eq. 4.12), com os vórtices da turbulência forte desempenhando um papel fundamental. Na próxima seção discutiremos a validade desta estimativa para o coeficiente de difusão por reconexão turbulenta.

\subsubsection{Comparação entre os efeitos da difusividade por reconexão magnética e da resistividade}

Como em Santos-Lima et al. (2010), podemos avaliar um coeficiente de difusão por reconexão turbulenta efetivo a partir de nossos modelos simulados compará-los com modelos resistivos, porém não turbulentos, que possuam uma grande resistividade Ôhmica. Por exemplo, considerando as mesmas condições iniciais que aquelas dos modelos subAlfvénicos N2c, N2d e N2e, realizamos várias simulações de modelos resistivos não turbulentos considerando diferentes valores de "super-resistividade Ôhmica. A Tabela 5.3 apresenta três grupos de modelos resistivos não turbulentos, N2cr, N2dr, e N2er cujas condições iniciais são as mesmas que as dos modelos N2c, N2d e N2e, respectivamente. A Figura 5.12 compara o modelo N2c com dois modelos resistivos N2cr1 e N2cr2 com as mesmas condições iniciais e super-resistividade Ôhmica $\eta_{O h m}=0.005$ u.c. e $\eta_{O h m}=0.001$ u.c., respectivamente. Vemos que o modelo resistivo com $\eta_{O h m}=0.001$ é o que reproduz melhor o modelo N2c. Isso nos sugere que a resistividade turbulenta efetiva do modelo N2c é $\eta_{\text {turb }} \approx 0.001$ u.c. Um procedimento similar para os modelos N2d e N2e permite-nos estimar também seus coeficientes de difusão turbulenta, os quais são apresentados na Tabela 5.4. Esta Tabela também mostra os valores correspondentes para a razão $\eta_{\text {turb }} / l_{\text {inj }} v_{\text {turb }}$ 
para estes modelos obtidos das simulações. De acordo com a eq. 4.17, esperaríamos que esta razão fosse menor do que 1 para turbulência sub-Alfvénica. Portanto, os valores obtidos são marginalmente consistentes com a previsão teórica, representada pela razão na última coluna (i.e., pelo número de Alfvén turbulento ao cubo). De fato, as relações analíticas para os coeficientes de difusão por reconexão turbulenta derivadas no Cap. 4, são ainda aproximações. Uma derivação mais correta depende de uma formulação mais exata da teoria de turbulência MHD que, como vimos no Cap. 4, é ainda deficiente (veja também Lazarian 2011).

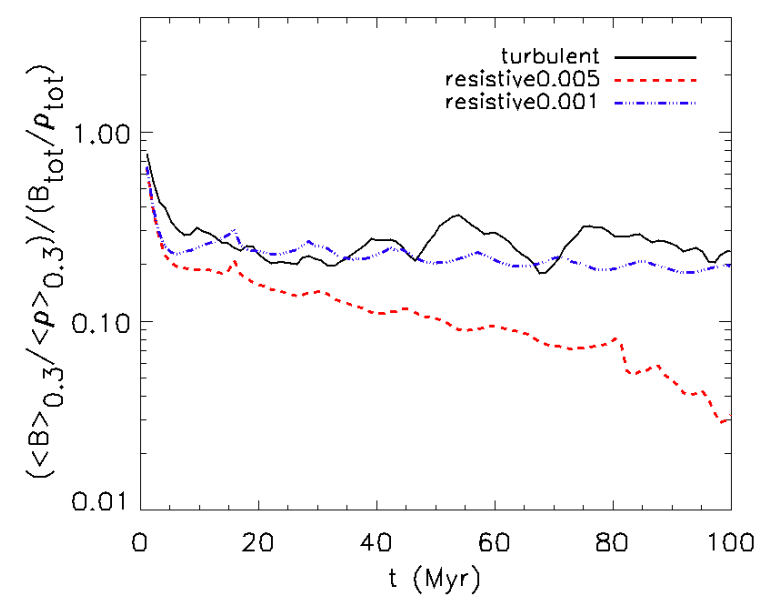

Figura 5.12: Evolução temporal da razão entre o campo magnético e a densidade média na região do núcleo da nuvem com um raio $r_{c}=0.3 \mathrm{pc}$, normalizado pelo valor médio desta sobre a nuvem toda, para o modelo turbulento N2e (linha sólida preta); o modelo resistivo N2er1 com $\eta_{O h m}=0.001$ u.c. (linha ponto-tracejada azul); e o modelo resistivo N2er2 com $\eta_{O h m}=0.005$ u.c. (linha vermelha tracejada). Notamos que o modelo resistivo N2er1 é comparável ao modelo turbulento N2e.

\subsubsection{Potencial gravitacional cilíndrico versus esférico}

Outro resultado importante é o fato de que na presença de um campo gravitacional esférico mais realista na nuvem o transporte de fluxo magnético é menos eficiente do que na presença de um campo cilíndrico (como o empregado em Santos-Lima et al. 2010). 
Tabela 5.3: Parâmetros para os modelos resistivos com as mesmas condições iniciais que os modelos sub-Alfvénicos N2c, N2d e N2e

\begin{tabular}{lccc}
\hline \hline Modelo & $\mathrm{n}\left(\mathrm{cm}^{-3}\right)$ & $\beta$ & $\eta_{\text {Ohm }}$ (c.u. $)$ \\
\hline N2cr1 & 90.0 & 1.0 & 0.001 \\
N2cr2 & 90.0 & 1.0 & 0.005 \\
N2cr3 & 90.0 & 1.0 & 0.01 \\
N2cr4 & 90.0 & 1.0 & 0.5 \\
N2cr5 & 90.0 & 1.0 & 0.1 \\
N2dr1 & 80.0 & 1.0 & 0.001 \\
N2dr2 & 80.0 & 1.0 & 0.005 \\
N2dr3 & 80.0 & 1.0 & 0.01 \\
N2er1 & 90.0 & 0.3 & 0.0005 \\
N2er2 & 90.0 & 0.3 & 0.001 \\
N2er3 & 90.0 & 0.3 & 0.005 \\
\hline
\end{tabular}

Tabela 5.4: O coeficiente de difusividade para os modelos sub-Alfvénicos

\begin{tabular}{llll}
\hline \hline Modelo & $\eta_{\text {turb }}($ u.c. $)$ & $\eta_{\text {turb }} / v_{\text {turb }} l_{\text {inj }}$ & $\left(v_{\text {turb }} / v_{A}\right)^{3}$ \\
\hline & & & \\
N2c & $\sim 0.001$ & $\sim 0.0024$ & $\sim 0.229$ \\
$\mathrm{~N} 2 \mathrm{~d}$ & $\sim 0.001$ & $\sim 0.0016$ & $\sim 0.096$ \\
$\mathrm{~N} 2 \mathrm{e}$ & $\sim 0.001$ & $\sim 0.0020$ & $\sim 0.315$ \\
\hline
\end{tabular}

Como vimos, isto já era esperado visto que em um potencial esférico todo o gás é puxado para um ponto central, enquanto que em um potencial cilíndrico, o gás é puxado para um eixo central ao longo do cilindro, fazendo com que o desacoplamento do gás com o fluxo magnético, o qual é induzido pela difusão por reconexão turbulenta, seja mais efetivo neste 
último caso. Portanto, devemos concluir que os resultados do estudo anterior, baseado em nuvens turbulentas cilíndricas realizado por Santos-Lima et al. (2010), superestimou o transporte de fluxo magnético devido à difusão por reconexão. Assim, por exemplo, para o modelo R1 com um potencial esférico (painéis superiores da Figura 5.2) a razão entre o campo magnético e a densidade diminuiu um fator 0.11 após 8 intervalos de tempo dinâmico, enquanto que o mesmo modelo com um potencial cilíndrico em Santos-Lima et al. (2010) (modelo D2) diminuiu muito mais, de um fator 0.65, no mesmo intervalo de tempo.

\subsubsection{Os efeitos da auto-gravidade sobre o transporte de fluxo magnético devido à difusão por reconexão turbulenta}

A comparação dos modelos sem auto-gravidade com os modelos auto-gravitantes revelou que a auto-gravidade pode ajudar significativamente o desacoplamento entre o gás e o fluxo magnético devido à difusão por reconexão, em particular nos estágios finais do colapso da nuvem. Um exemplo crítico é o modelo auto-gravitante N2b (Figuras 5.2 e 5.3), no qual a difusão por reconexão gera um núcleo super-crítico, enquanto que sua contra-partida sem auto-gravidade, o modelo R2 (Figuras 5.10 e 5.11), é incapaz de desenvolver um núcleo super-crítico.

Um aumento na auto-gravidade (que é fornecido pelo aumento na densidade inicial do gás da nuvem) melhora o transporte turbulento de fluxo magnético. Se a densidade do gás na nuvem é grande o suficiente $\left(n_{0}>50 \mathrm{~cm}^{-3}\right)$, seus efeitos parecem ser mais importantes do que o do potencial gravitacional estelar (para $M_{\star} \sim 41 \mathrm{M}_{\odot}$ ) para ajudar o desacoplamento entre o gás e o campo magnético (veja os modelos N2b e N3 nas Figuras 5.2 e 5.3 e 5.7 e 5.5). Contudo, para uma dada intensidade da turbulência, se aumentamos a densidade inicial ou o potencial gravitacional estelar indefinidamente, então, eventualmente o potencial gravitacional total se tornará tão grande que irá neutralizar a capacidade da difusão por reconexão de desacoplar o fluxo magnético do gás denso e assim nenhum transporte eficiente de fluxo magnético ocorrerá para fora do núcleo da nuvem. Nesse caso, a maior parte do fluxo magnético será dragado pelo gás colapsante. Vemos 
este efeito, por exemplo, quando aumentamos a densidade inicial da nuvem no modelo $\mathrm{N} 2 \mathrm{e}$ (o qual tem inicialmente $\beta=1.0, M_{\star} \sim 41 \mathrm{M}_{\odot}$, e $n_{0}=80 \mathrm{~cm}^{-3}$ ) para $n_{0}=90 \mathrm{~cm}^{-3}$ no modelo N2c (veja as Figuras 5.4 e 5.5). Enquanto o primeiro mostra evidências de algum transporte de fluxo magnético e desenvolve um núcleo aproximadamente crítico, o segundo falha completamente. Similarmente, para o modelo N2b (o qual possui $\beta=3.0$, $M_{\star} \sim 41 \mathrm{M}_{\odot}$, e $n_{0}=90 \mathrm{~cm}^{-3}$ ), a turbulência trans-sônica e trans-Alfvénica permite a formação de um núcleo super-crítico como indicado nas Figuras 5.2 e 5.3. Contudo, se aumentamos sua densidade inicial para $100 \mathrm{~cm}^{-3}$, o potencial gravitacional total torna-se tão grande e o colapso fica tão rápido que a reconexão magnética turbulenta torna-se ineficaz para desacoplar o fluxo magnético do núcleo denso. Por outro lado, com a densidade aumentada $\left(100 \mathrm{~cm}^{-3}\right)$, reduzimos a massa estelar para $\mathrm{M}_{\star} \sim 27 \mathrm{M}_{\odot}$ como no modelo N3 (Figuras 5.7 e 5.8), tal que a massa total seja aproximadamente a mesma que no modelo N2b, então o transporte de fluxo fica evidente e um núcleo super-crítico também se desenvolve. Porém, uma nova redução na massa total (como em N4) novamente impede a formação de um núcleo super-crítico, pois neste caso a queda do gás para o centro torna-se tão lenta que a turbulência de fato ajuda a espalhar o material do núcleo.

\subsubsection{Os efeitos das condições iniciais da nuvem}

Todos os resultados discutidos acima foram encontrados para nuvens que tinham uma densidade inicial uniforme e estavam fora do equilíbrio magneto-hidrostático quando a turbulência foi injetada. Testamos também um modelo começando em equilíbrio magnetohidrostático tendo uma densidade estratificada, sendo a densidade central e todas as outras condições iniciais iguais às do modelo de referência (N2b) (veja o modelo E1 na Figura 5.6). Como o modelo N2b, o modelo E1 também apresenta um transporte de fluxo magnético para fora do núcleo devido à reconexão turbulenta eficiente. Contudo, a massa total muito menor de E1 devido à estratificação da nuvem (com uma fração de 0.43 da massa total do modelo N2b) a impede de formar um núcleo super-crítico dentro do intervalo de tempo analisado.

Os resultados acima indicam que a formação de um núcleo super-crítico é regulada 
por uma complexa combinação entre gravidade, auto-gravidade, intensidade do campo magnético e turbulência aproximadamente trans-sônica e trans-Alfvénica. Embora tenhamos encontrado que a difusão por reconexão turbulenta é muito eficiente para remover o fluxo magnético da maioria dos núcleos colapsantes testados aqui, somente alguns poucos foram bem sucedidos em desenvolver núcleos aproximadamente críticos ou super-críticos (veja os modelos N2b, N2e, e N3), os quais serão capazes de colapsar e formar estrelas. Ou seja, para as condições das nuvens investigadas aqui, a formação de núcleos supercríticos é restrita a um intervalo limitado de parâmetros, como deveríamos esperar, já que observações prevem uma baixa eficiência de formação estelar como discutido no Cap. 1 (veja também Mac Low \& Klessen 2004; Leão et al. 2009; Vazquez-Semadeni et al. 2011).

Para resumir, nossos resultados sugerem que o transporte de fluxo por reconexão turbulenta permitirá nuvens inicialmente sub-críticas tornarem-se aproximadamente críticas ou super-críticas para nuvens (ou glóbulos) com valores iniciais de $\beta \sim 1$ a 3 , densidades da nuvem $50<n_{0}<100 \mathrm{~cm}^{-3}$, quando consideramos massas estelares $M_{\star} \sim 41 \mathrm{M}_{\odot}$, e $100<n_{0}<140 \mathrm{~cm}^{-3}$, quando consideramos massas estelares $M_{\star} \sim 27 \mathrm{M}_{\odot}$, implicando em glóbulos com massas totais $M_{\text {tot }} \lesssim 120 \mathrm{M}_{\odot}$. Para densidades menores, as nuvens são fragmentadas pela força da turbulência injetada e nenhum núcleo se forma ${ }^{5}$. Para densidades maiores, os efeitos da auto-gravidade são tão fortes que o núcleo colapsa dragando o campo magnético, de modo que não há nenhum transporte significativo de fluxo magnético. Dos 9 modelos auto-gravitantes estudados aqui, 4 formam núcleos aproximadamente críticos ou supercríticos (N1, N2b, N2e e N3) e 2 formam núcleos sub-críticos (N2a e E1), todos com evidências de transporte de fluxo magnético. Os 3 modelos remanescentes (N2c, N2d e N4) não apresentam qualquer evidência de transporte de fluxo magnético por difusão por reconexão (em virtude do fato de a nuvem possuir um campo magnético inicial muito forte ou à turbulência ser muito forte, como descrito na Secção 5.3). A Tabela 5.5 lista as condições finais dos núcleos formados para todos os modelos simulados.

\footnotetext{
${ }^{5}$ É possível que este efeito esteja um pouco subestimado devido ao emprego de uma equação isotérmica de estado para as nuvens (veja Cap. 6).
} 


\subsubsection{Comparação de nossos resultados com as observações}

Um modo de se tentar compreender o processo pelo qual as estrelas formam-se é medir a intensidade dos campos magnéticos em nuvens moleculares, a fim de verificar se estes são fracos o suficiente (ou seja, a nuvem é supercrítica) ou fortes (ou seja, a nuvem é subcrítica). Medições do efeito Zeeman recentes de núcleos de nuvens escuras e envelopes realizadas por Crutcher et al. (2009, 2010, veja também Troland \& Crutcher 2008) trouxeram novos resultados e desafios para a teoria de transporte de fluxo magnético por difusão ambipolar. Eles obtiveram a razão massa-fluxo magnético entre o núcleo da nuvem e o envelope, $R=\left(M_{c} / \Phi_{c}\right) /\left(M_{e} / \Phi_{e}\right)$, bem como a razão massa-fluxo magnético entre o núcleo e a nuvem toda (núcleo+envelope), $R^{\prime}=\left(M_{c} / \Phi_{c}\right) /\left(M_{c+e} / \Phi_{c+e}\right)$, e encontraram que estas razões são menores do que um para as quatros nuvens investigadas (B1, B2172, L1448CO e L1544). A difusão ambipolar requer que a razão massa-fluxo magnético no núcleo seja maior do que a da nuvem toda, implicando em $R^{\prime}>1$, o qual está em contradição com os resultados encontrados por Crutcher et al.

Embora as condições dos núcleos e dos envelopes das nuvens investigados por Crutcher et al. sejam um pouco distintas daquelas obtidas nas simulações deste estudo, podemos ao menos fazer comparações qualitativas com nossos resultados. Apesar de mais densos e mais magnetizados, seus núcleos são todos sub-críticos ou aproximadamente críticos (i.e., eles têm razões massa-fluxo magnético em relação ao valor crítico no intervalo $\mu_{c r i t}=$ 0.45 - 1.15). Os núcleos formados em nossos modelos com evidências de transporte turbulento de fluxo possuem razões massa-fluxo magnético média final $\mu_{\text {crit }}=0.15-5.25$. A maioria destes núcleos possui razões $R$ e $R^{\prime}$ que são consistentes com as inferidas por Crutcher et al. (2009), com a exceção dos núcleos formados nos modelos N1 e Nb2 (veja a Tabela 5.5, onde estas razões são apresentadas para o tempo final de todos os nossos modelos). De fato, observacionalmente sabe-se que o nível de turbulência diminui nos núcleos. Vimos que a difusão por reconexão desacelera com a diminuição da velocidade turbulenta (Santos-Lima et al. 2010; de Gouveia Dal Pino et al. 2011; Lazarian 2011; Lazarian et al. 2012a) de modo que deveríamos esperar um transporte de fluxo magnético mais lento para fora do núcleo quando comparado com o envelope. Este comportamento 
é de fato detectado nos núcleos formados neste estudo.

No caso particular do modelo $\mathrm{N} 2 \mathrm{~b}$, as razões encontradas $R$ e $R^{\prime}$ são menores do que a unidade até o núcleo tornar-se altamente super-crítico, deste ponto em diante $R$ e $R^{\prime}$ ficam maiores do que a unidade devido ao aumento descontrolado/desenfreado da razão massa-fluxo magnético do núcleo colapsante (a razão massa-fluxo magnético máxima do núcleo $\mu_{\text {crit }, c} \sim 40.7$, como mostrado na Tabela 5.5 e na Figura 5.3).

As Figuras 5.13 e 5.14 ilustram o transporte das linhas de campo magnético do núcleo colapsante para o envelope para o modelo N2b. Enquanto a Figura 5.13 compara este modelo turbulento com sua contra-partida não turbulenta no passo de tempo final $(\mathrm{t}=$ 100 Myrs), a Figura 5.14 mostra a evolução temporal deste modelo turbulento até o início do colapso do núcleo por volta de 90 Myrs quando então, a porção de fluxo magnético que não foi transportada difusivamente para o envelope circundante é advectada para o centro pelo gás colapsante, causando um grande aumento na intensidade do campo magnético nesta região.

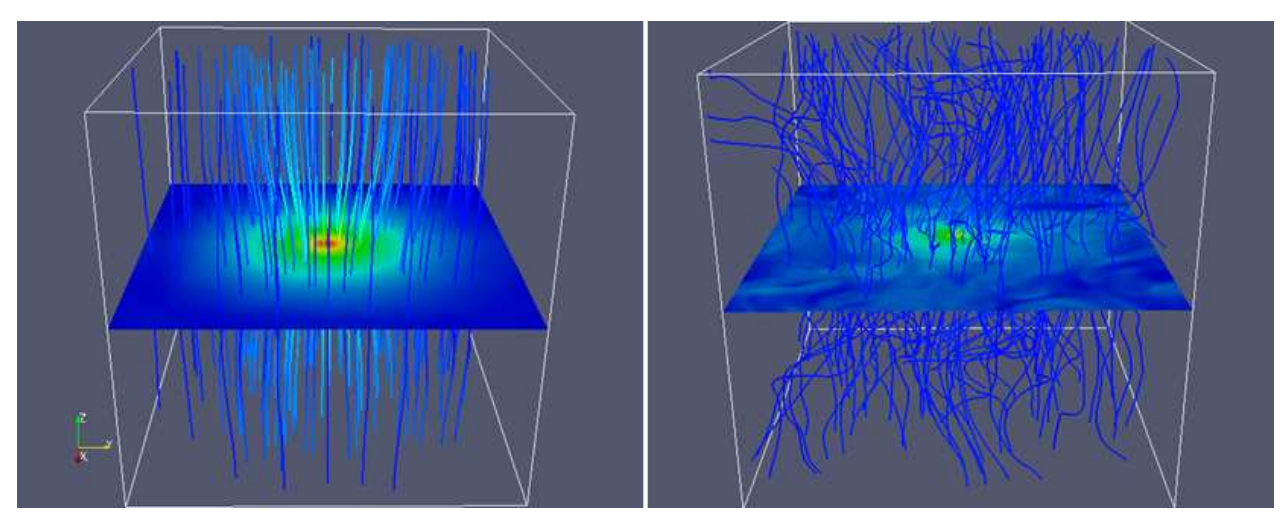

Figura 5.13: Distribuição das linhas de campo magnético e corte equatorial da distribuição logarítmica da densidade para o modelo colapsante N2b. O painel à direita mostra o modelo turbulento enquanto que o painel à esquerda mostra sua contra-partida laminar. O tempo apresentado é 100 Myrs para ambos os modelos. O núcleo central possui uma densidade $50 \mathrm{~cm}^{-3}$. Foram graficadas 100 linhas de campo magnético igualmente espaçadas dentro de um raio de $\sim 1.3 \mathrm{pc}$.

No caso do modelo $\mathrm{N} 1$, as razões $R$ e $R^{\prime}$ são menores do que 1 por vários passos de 
tempo. Após 68 Myrs, um aumento muito rápido da densidade central causado pelo alto potencial gravitacional central neste modelo faz com que $R^{\prime}$ e $R$ aumentem seus valores para maiores do que 1 (a mesma situação pode ser aplicada para o modelo sem autogravidade R1). Portanto, os três núcleos terminam com $R$ e $R^{\prime}>1$ porque já estão colapsando para formar proto-estrelas, N2b devido ao domínio da auto-gravidade e N1 (e R1) devido ao forte potencial central. Os outros núcleos super-críticos (N2e e N3) ainda possuem valores de $R$ e $R^{\prime}<1$ no tempo final simulado, e portanto, comparáveis àqueles obtidos para os núcleos observados por Crutcher et al.

Notemos finalmente, que os valores inferidos das observações para $R$ e $R^{\prime}$ por Crutcher et al. estão sujeitos a incertezas significativas devido a limitações nas medições e a suposições simplificadas (veja Mouschovias \& Tassis 2010). Portanto, embora encorajadores, os resultados das comparações acima devem ser vistos com cautela. Além disso, mais dados e mais estimativas observacionais precisas são ainda necessárias.

\subsection{Observações finais}

O mecanismo aqui discutido de difusão por reconexão pode apresentar a última peça que falta para a construção de um novo paradigma na formação de estrelas, onde a turbulência desempenha um papel central (veja também Lazarian 2011). Este estudo numérico, juntamente com o anterior de Santos-Lima et al. (2010) investigaram este novo mecanismo de transporte de campo magnético, tendo como foco principal os estágios iniciais da formação estelar. Recentemente, Santos-Lima et al. (2012a, 2012b) também investigaram este mecanismo nos estágios finais, durante a formação dos discos proto-estelares. Estes autores demonstraram, também por meio de simulações 3D MHD (similares às aqui apresentadas), que a difusividade devido a reconexão magnética na presença de turbulência também é capaz de transportar o excesso de fluxo magnético para as regiões externas do disco em formação em escalas de tempo compatíveis com o colapso do núcleo. Em apenas $\sim 30$ mil anos, mostraram que um disco girante forma-se ao redor de uma proto-estrela com um perfil aproximadamente Kepleriano, como exigido pelas observações. Uma vez que a turbulência MHD deve estar presente também em discos proto-estelares, este é um meca- 
Tabela 5.5: Quantidades finais obtidas para os núcleos e envelopes formados. Densidade central, $n_{c}$, e campo magnético na direção z, $B_{c}$, razão entre as energias turbulenta e magnética, $E_{\text {turb }} / E_{\text {mag }}$, massa gasosa, $M_{c}$, fluxo magnético, $\Phi_{c}$, e razão massa-fluxo magnético máxima em relação ao valor crítico, $\mu_{c r i t, c}$ para os núcleos formados $\left(r_{c} \leq 0.3\right.$ $\mathrm{pc})$.

\begin{tabular}{lcccccc}
\hline \hline & & & & & \\
Modelo & $n_{c}\left(\mathrm{~cm}^{-3}\right)$ & $B_{c}(\mu \mathrm{G})$ & $E_{\text {turb }} / E_{\text {mag }}$ & $M_{c}\left(\mathrm{M}_{\odot}\right)$ & $\Phi_{c}\left(10^{31}{\mathrm{G} . c{ }^{2}}^{2}\right)$ & $\mu_{\text {crit }, c}$ \\
\hline $\mathrm{R} 1$ & $3.8 \times 10^{3}$ & 1.92 & 4.83 & 0.7 & 0.20 & 1.32 \\
$\mathrm{R} 2$ & $4.2 \times 10^{3}$ & 4.04 & 1.68 & 2.0 & 0.62 & 0.96 \\
$\mathrm{R} 3$ & 994.7 & 3.32 & 3.55 & 1.1 & 0.43 & 0.73 \\
\hline $\mathrm{N} 1$ & $3.5 \times 10^{3}$ & 2.60 & 4.53 & 0.6 & 0.23 & 1.2 \\
$\mathrm{~N} 2 \mathrm{a}$ & 505.3 & 1.44 & 6.44 & 0.2 & 0.15 & 0.6 \\
$\mathrm{~N} 2 \mathrm{~b}$ & $1.1 \times 10^{7}$ & 89.6 & 14.5 & 13.2 & 0.84 & 40.7 \\
$\mathrm{~N} 2 \mathrm{c}$ & $5.6 \times 10^{3}$ & 8.75 & 2.42 & 1.4 & 0.68 & 0.7 \\
$\mathrm{~N} 2 \mathrm{~d}$ & $2.9 \times 10^{3}$ & 4.14 & 6.48 & 1.0 & 1.43 & 0.2 \\
$\mathrm{~N} 2 \mathrm{e}$ & $1.3 \times 10^{3}$ & 9.00 & 0.81 & 1.2 & 0.92 & 1.5 \\
$\mathrm{~N} 3$ & $2.3 \times 10^{3}$ & 3.79 & 7.72 & 1.7 & 0.31 & 2.0 \\
$\mathrm{~N} 4$ & $1.3 \times 10^{3}$ & 3.29 & 2.48 & 1.4 & 0.51 & 0.8 \\
$\mathrm{E} 1$ & 111.6 & 0.85 & 0.77 & 0.1 & 0.11 & 0.3 \\
\hline
\end{tabular}

nismo natural e rápido para remover o excesso de fluxo magnético e permitir a formação destes discos. Portanto, reunidos, todos os estudos acima permitiram testar este novo paradigma, demonstrando que a remoção de fluxo magnético de nuvens moleculares devido à difusão por reconexão é muito eficiente. Além disso, sugere uma revisão do verdadeiro papel que a difusão ambipolar (e outros mecanismos de transporte) podem desempenhar nos processos de formação estelar e planetária.

Finalmente, devemos ressaltar que neste estudo nos concentramos na evolução de nuvens auto-gravitantes isotérmicas com estrelas embebidas gerando um potencial gravitacional externo. Da mesma forma, alguns dos núcleos de nuvens escuras observados que mencionamos acima realmente contém estrelas embebidas neles (e.g., as nuvens B1 
Tabela 5.6: Continuação da Tabela 5.5. Quantidades finais obtidas para os núcleos e envelopes formados. Massa gasosa, $M_{e}$, e fluxo magnético, $\Phi_{e}$, para os envelopes formados; razão massa-fluxo magnético entre o núcleo e o envelope da nuvem, $R$, razão massa-fluxo magnético entre o núcleo e a nuvem toda (núcleo+envelope), $R^{\prime}$, no passo de tempo final $(t \sim 100$ Myrs). Modelos N1, N2b, N2e, N3 e R1 desenvolvem um núcleo supercrítico ou aproximadamente crítico. Todos os modelos exceto N2c, N2d, e N4, apresentam evidências de transporte de fluxo magnético devido à difusão por reconexão turbulenta.

\begin{tabular}{lcccc}
\hline \hline Modelo & $M_{e}\left(\mathrm{M}_{\odot}\right)$ & $\Phi_{e}\left(10^{31} \mathrm{G} \cdot \mathrm{cm}^{2}\right)$ & $R$ & $R^{\prime}$ \\
\hline $\mathrm{R} 1$ & 7.7 & 3.54 & 1.52 & 1.47 \\
$\mathrm{R} 2$ & 73.7 & 10.6 & 0.46 & 0.48 \\
$\mathrm{R} 3$ & 83.0 & 11.2 & 0.33 & 0.34 \\
\hline $\mathrm{N} 1$ & 7.8 & 0.35 & 1.29 & 1.27 \\
$\mathrm{~N} 2 \mathrm{a}$ & 8.2 & 3.73 & 0.73 & 0.74 \\
$\mathrm{~N} 2 \mathrm{~b}$ & 62.5 & 11.1 & 2.80 & 2.49 \\
$\mathrm{~N} 2 \mathrm{c}$ & 74.3 & 19.4 & 0.53 & 0.54 \\
$\mathrm{~N} 2 \mathrm{~d}$ & 74.7 & 35.4 & 0.34 & 0.35 \\
$\mathrm{~N} 2 \mathrm{e}$ & 66.1 & 18.2 & 0.35 & 0.36 \\
$\mathrm{~N} 3$ & 82.4 & 11.8 & 0.79 & 0.79 \\
$\mathrm{~N} 4$ & 82.7 & 11.8 & 0.38 & 0.39 \\
$\mathrm{E} 1$ & 2.2 & 1.93 & 0.39 & 0.41 \\
\hline
\end{tabular}

e L1448CO; Bachiller, Menten, \& del Rio-Alvarez 1990; Volgenau et al. 2006), de modo que nosso estudo é bastante realista.

Os resultados apresentados neste capítulo foram descritos em dois trabalhos, um já publicado (veja Ap. D) e o outro recentemente submetido para publicação (Ap. E). 


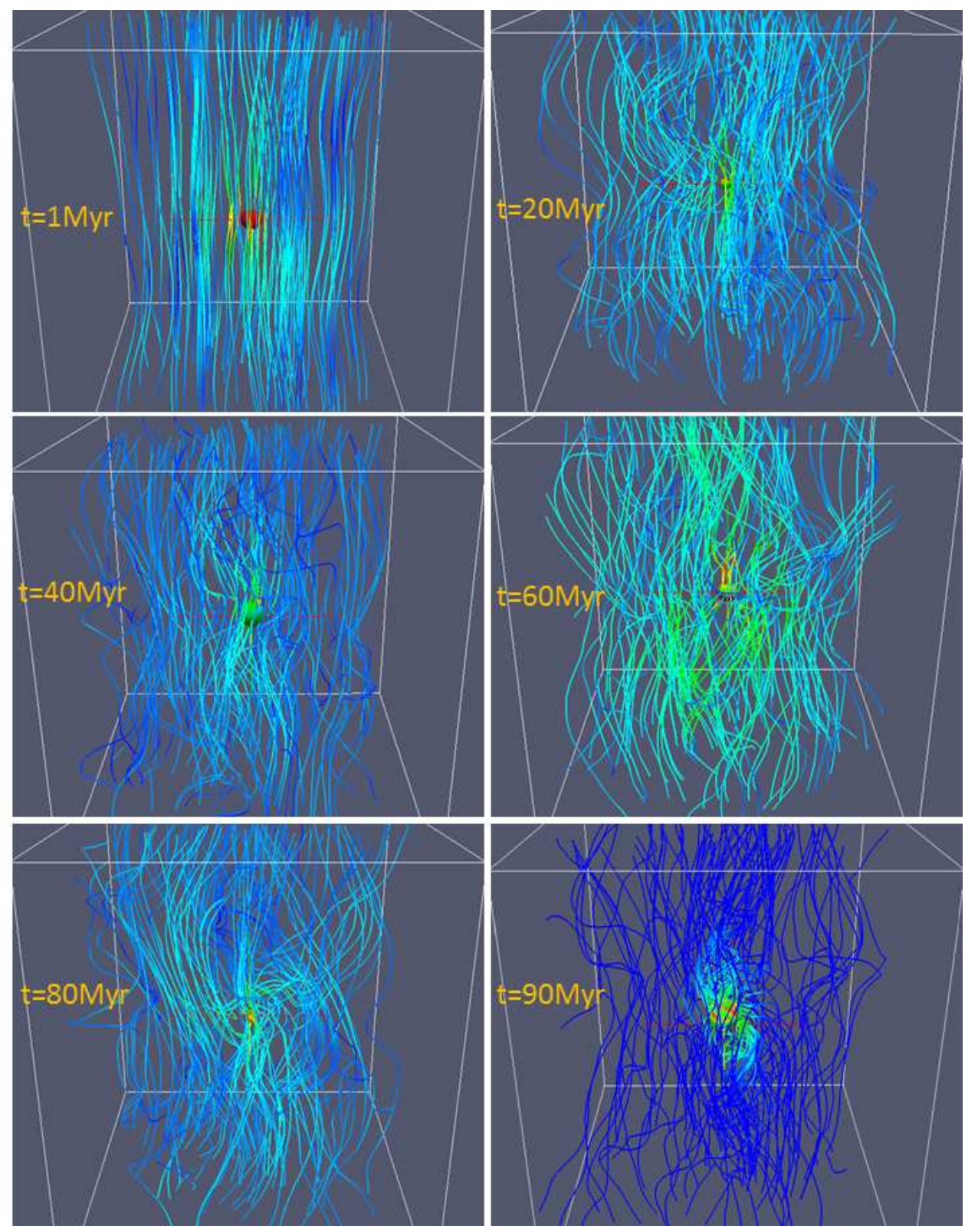

Figura 5.14: O mesmo que a Figura 5.13, mas mostrando a evolução temporal das linhas de campo magnético do modelo turbulento N2b a partir de $t=1$ até 90 Myrs. Vemos claramente como a turbulência transporta difusivamente as linhas de campo magnético à medida que o tempo evolui. Em t=90 Myrs as linhas que não foram transportadas para as regiões externas da nuvem são advectadas para o centro pelo gás colapsante causando um aumento local na intensidade do campo magnético. A escala de cor indica a intensidade do campo magnético, com o vermelho representando o valor máximo e o azul o mínimo. 


\section{Capítulo 6}

\section{Conclusões e Perspectivas}

"A conclusion is the place where you got tired thinking."

(Martin H. Fischer)

Neste Capítulo apresentaremos um sumário dos resultados mais importantes desta tese onde, numa primeira parte deu-se ênfase ao estudo do processo de formação estelar induzido pelo impacto de nuvens interestelares por remanescentes de supernovas - um dos principais agentes de injeção de energia e turbulência no meio interestelar. Na segunda parte deste trabalho, o enfoque foi o estudo da presença de turbulência magneto-hidrodinâmica, propriamente, no interior das nuvens interestelares e seus efeitos na remoção de campos magnéticos e na formação estelar. Também neste capitulo apresentaremos as perspectivas para dar continuidade a esses estudos.

\subsection{Conclusões - Formação Estelar induzida por Cho- ques de SN}

Considerando as condições físicas que são relevantes para a formação estelar induzida pelas interações entre choques de SNs e nuvens neutras difusas magnetizadas derivamos três diferentes condições necessárias para a indução de instabilidade e colapso gravitacional na nuvem e consequente formação estelar. A primeira determina o limite de Jeans para o material chocado da nuvem sob a ação do impacto do RSN (equação 2.42). A segunda 
estabelece que a frente de choque que se propaga dentro da nuvem deve ter energia suficiente para atravessar a nuvem antes de parar em seu interior devido a perdas radiativas (equação 2.51). Deste modo, o choque poderá injetar o máximo de energia no material da nuvem de forma a comprimi-lo eficientemente. E a terceira condição é que esta mesma frente de choque não seja forte demais a ponto de destruir a nuvem completamente, fazendo com que o gás se disperse no meio interestelar antes de tornar-se gravitacionalmente instável (equação 2.48). Com isso, construímos diagramas do raio do RSN, $R_{R S N}$, versus a densidade inicial da nuvem, $n_{c}$, nos quais essas condições limitam uma zona sombreada no espaço paramétrico, onde a formação de estrelas é possível. A validade desses diagramas foi ainda testada por meio de simulações numéricas 3D MHD de colisões entre um RSN e uma nuvem (Caps. 2 e 3).

Aplicações desses resultados a regiões reais de formação estelar em nossa própria galáxia revelaram que estas poderiam ter sido induzidas por ondas de choque de SN (por exemplo, as regiões de formação estelar da Grande Concha de CO e da Nuvem Periférica $2)$.

A inclusão de campos magnéticos típicos na nuvem leva a uma diminuição da região permitida para formação estelar nos diagramas, porém não muito relevante para as intensidades médias observadas em nuvens neutras (da ordem de $6 \mu \mathrm{G}$ ). Calculamos também a eficiência de formação estelar (sfe) para essas interações entre SNRs e nuvens neutras difusas. Notamos que para uma dada densidade inicial de nuvem considerada, $n_{c}$, a sfe está bem determinada dentro dos limites estabelecidos pelos vínculos de não destruição da nuvem e de penetração do choque acima descritos. Nos gráficos de sfe observa-se que a presença do campo magnético desloca a área sombreada para raios $R_{R S N}$ menores. Os diagramas mostraram que o espaço de parâmetros destas interações RSN-nuvem que permitem a indução de formação estelar é bem estreito. Em particular, ao aumentarmos a intensidade do campo para $10 \mu \mathrm{G}$ vemos que a região sombreada fica restrita a valores de sfe bem menores que o máximo observado em regiões de intensa formação estelar na Galáxia. Isto sugere que o mecanismo de indução de formação estelar pelo impacto de RSNs em nuvens é compatível com as baixas eficiências medidas de formação estelar observadas na Galáxia (em média < 0.3). Além do mais, sua baixa eficiência intrínseca para 
formar estrelas (com uma $s f e_{R S N}$ bem inferior à sfe média da Galáxia), sugere que esse mecanismo deve ser mais eficiente para induzir a formação de sub-estruturas e turbulência nas nuvens, do que para formar estrelas, propriamente (Cap. 3).

Os resultados desses estudos foram publicados em Leão et al. 2009 e uma cópia deste trabalho encontra-se no Apêndice C.

\subsection{Conclusões - Formação estelar em nuvens turbu- lentas e o transporte de Fluxo Magnético}

Através de simulações numéricas magneto-hidrodinâmicas tridimensionais de glóbulos de nuvens frias auto-gravitantes em presença de um campo magnético externo uniforme e um campo gravitacional esférico gerado por um grupo de estrelas no centro da nuvem, estudamos os efeitos da turbulência no colapso gravitacional das mesmas. Em particular, examinamos a difusão dos campos magnéticos por reconexão magnética turbulenta (Cap. 4). Na maiorias dos modelos testados o sistema foi iniciado já fora de equilíbrio magnetohidrostático mas, para comparação realizamos também um teste iniciando em equilíbrio obtido pela relaxação do sistema. Testes sem auto-gravidade foram também realizados para comparação com o trabalho anterior de Santos-Lima et al. (2010) onde empregaramse nuvens mais simples, sem auto-gravidade e com simetria cilíndrica.

Os resultados desse estudo revelaram que, em presença de turbulência aproximadamente transônica e trans-Alfvénica, esse mecanismo é suficiente para transportar de modo eficiente o excesso de fluxo magnético do núcleo da nuvem para o seu envelope, levando o núcleo a colapsar gravitacionalmente para formar estrelas. Esse mecanismo parece ser bem mais eficiente que a denominada difusão ambipolar, e não apresenta as dificuldades desta, recentemente discutidas na literatura. A difusão por reconexão magnética turbulenta constitui, portanto, um novo paradigma para a teoria de formação estelar.

Os resultados sugerem que a turbulência pode mudar substancialmente o perfil do fluxo magnético e da razão massa-fluxo magnético sem qualquer efeito da difusão ambipolar. Para quatro dos nove modelos numéricos auto-gravitantes testados aqui encontramos um 
transporte eficiente de fluxo magnético para as regiões externas menos densas da nuvem e a formação de um caroço denso aproximadamente crítico ou super-crítico, mostrando que é possível a formação destes a partir de nuvens sub-críticas, considerando apenas os efeitos da turbulência. A maioria destes caroços supercríticos formados nas simulações podem ser comparados com as observações de Crutcher et al. (2009). Os modelos simulados N1 e N2b, contudo, atingiram um estado final no qual o núcleo torna-se super-crítico, colapsando para formar uma proto-estrela.

A auto-gravidade tende a tornar mais eficiente o desacoplamento entre o gás colapsante e o fluxo magnético que flui para as regiões mais externas do envelope da nuvem, impedindo a dispersão do gás pela turbulência. Porém, se aumentarmos demais a densidade do gás (auto-gravidade), o tempo de queda livre ficará tão pequeno que a turbulência não terá tempo de agir para difundir o campo magnético o suficiente e este será dragado juntamente com o gás colapsante e eventualmente irá impedir a formação de um núcleo super-crítico. Um potencial central forte também facilita o desacoplamento entre o gás e o campo magnético, mas, da mesma forma que a auto-gravidade, um potencial central muito forte irá diminuir o tempo de queda livre tanto que o campo também será arrastado para o centro juntamente com o gás antes que a turbulência possa remover o excesso de fluxo. Os estudos numéricos também mostraram que quanto maior a razão entre a pressão térmica e a magnética, $\beta$, (mais fraco o campo magnético) mais eficiente é a difusão do campo magnético. Contudo, se aumentarmos demais a intensidade deste, tornando a turbulência altamente sub-Alfvénica, esta não será capaz de mudar a topologia ou difundir o campo magnético, e este último dificultará o colapso gravitacional.

Finalmente, verificamos que existe uma pequena faixa de parâmetros para as nuvens frias difusas para os quais tem-se um transporte eficiente de fluxo magnético devido à difusão por reconexão turbulenta, permitindo ao mesmo tempo o colapso gravitacional e a formação de núcleos super-críticos nas nuvens. Em presença de turbulência aproximadamente trans-sônica e trans-Alfvénica, as condições iniciais para as nuvens formarem caroços críticos ou super-críticos se restringem a: razão pressão magnéticapressão térmica, $\beta \sim 1$ a 3 , razões entre a energia turbulenta e a energia magnética $E_{\text {turb }} / E_{\text {mag }} \sim 1.62$ to 2.96 , e densidades da nuvem $50<n_{0}<100 \mathrm{~cm}^{-3}$, quando con- 
sideramos massas estelares $M_{\star} \sim 41 \mathrm{M}_{\odot}$, e $100<n_{0}<140 \mathrm{~cm}^{-3}$, quando consideramos massas estelares $M_{\star} \sim 27 \mathrm{M}_{\odot}$, implicando uma massa total da nuvem (gás + estrelas) $M_{\text {tot }} \lesssim 120 \mathrm{M}_{\odot}$. O fato de termos obtido um espaço paramétrico limitado para a formação desses caroços super-críticos é também uma baixa eficiência de formação estelar (sfe) observada na Galáxia.

Os resultados dos estudos descritos nos Caps. 4 e 5 foram em parte publicados em de Gouveia Dal Pino et al. (2012) e estão apresentados na íntegra em artigo recentemente submetido para publicação (Leão et al. 2012). Cópia destes encontram-se nos Apêndices D e E, respectivamente.

\subsection{Perspectivas}

Pretendemos dar continuidade aos estudos dos processos de formação estelar induzida por choques de SNs (Caps. 2 e 3) e outros agentes físicos do meio interestelar, para confrontação com as observações. Em particular, simulações numéricas magneto-hidrodinâmicas dessas interações incluindo os efeitos da auto-gravidade, tal como fizemos no estudo de nuvens turbulentas no Cap. 5, serão realizadas. Isto permitirá seguir o colapso gravitacional do caroço da nuvem por tempos bem maiores dentro do domínio computacional. Além disso, o emprego de resoluções numéricas ainda mais altas e de uma nova versão do nosso código numérico 3D MHD-Godunov incluindo rede adaptativa (veja página de Grzegorz Kowal - daphne.iag.usp.br/gitweb), permitirão acompanhar a evolução do material chocado dentro das nuvens e observar inclusive se há transporte de fluxo magnético por reconexão turbulenta devido à turbulência induzida pelo choque. Note-se que nesse caso, o mecanismo de turbulência induzido na nuvem é real (e não somente turbulência injetada artificialmente na nuvem, como adotado no estudo do Cap. 5). Esta é inicialmente supersônica, mas a compressão pelo choque, como vimos (Cap. 3), acarreta a formação de estruturas densas no material varrido pelo choque, particularmente no caroço da nuvem, e em alguns casos este torna-se gravitacionalmente instável e colapsa.

Outro ingrediente importante que será considerado na continuação de nossos estudos de formação estelar, é a inclusão das perdas radiativas do gás, ao invés de simplesmente 
utilizarmos a hipótese de gás isotérmico, como fizemos em todo este trabalho. A hipótese isotérmica, embora bastante conveniente e rápida para mimetizar os efeitos das perdas radiativas de um gás, pode em muitas situações "subestimar" os reais efeitos destas. Porém, como em estudos de formação estelar o resfriamento radiativo é um ingrediente fundamental na dinâmica e evolução do gás, a sua inclusão de modo mais realista deve ser considerada. Essa implementação será realizada no mesmo código 3D MHD-Godunov que empregamos nesta tese. Utilizaremos para tal, rotinas que já foram utilizadas em outros estudos pelo grupo (e.g. de Gouveia Dal Pino \& Benz 1993; Melioli \& de Gouveia Dal Pino 2004; Melioli et al. 2005; 2006; 2008; 2009) .

Finalmente, a comparação do mecanismo de indução de formação estelar por SNs com outros processos potencialmente importantes, como ondas de choque de ventos estelares e ondas de choque espirais de larga escala nos permitirá obter as eficiências relativas desses outros mecanismos.

Iremos ainda estender os estudos de nuvens turbulentas (Caps. 4 e 5) considerando diferentes vertentes. Por um lado, iremos explorar os efeitos do transporte de fluxo magnético por reconexão turbulenta também na evolução de nuvens inicialmente sem estrelas embebidas, isto é, sem a ação de um campo gravitacional externo, a fim de avaliar somente os efeitos da auto-gravidade isoladamente sobre o transporte. Por outro lado, iremos efetuar uma avaliação mais precisa do coeficiente de difusão por reconexão magnética turbulenta. Como observado no Cap. 4, essa avaliação não será simples, pois depende de uma formulação mais robusta da teoria de turbulência MHD em geral, a qual está ainda em construção, e constituirá portanto, um grande desafio para os próximos anos. 


\section{Apêndice A}

\section{Supernovas e seus remanescentes}

Uma supernova (SN) é o produto da explosão de uma estrela que cria um objeto extremamente luminoso. Esta explosão expele todo ou quase todo material da estrela a grandes velocidades gerando uma onda de choque no gás interestelar ao redor. Este choque varre o meio formando uma casca esférica de gás e poeira chamado remanescente de supernova $(\mathrm{RSN})$.

Para entender como SNs injetam energia e geram turbulência no meio interestelar devemos entender como são formadas as supernovas e seus remanescentes, e como se dá esta injeção de energia no meio. Revisaremos aqui os tipos de supernovas, suas características e a formação do remanescente que efetivamente impacta uma nuvem (veja também Leão 2007).

\section{A.1 Tipos de Supernovas}

Existem duas possíveis formas de uma estrela se transformar em supernova. Nas supernovas tipo I (SNI), uma estrela anã branca acreta material de uma estrela companheira até atingir a massa crítica e sofrer combustão geral. Na supernova tipo II (SNII), a explosão é resultado do fim do ciclo de vida de uma estrela massiva. Nesta classificação tradicional as SNs Tipo I são caracterizadas principalmente por não apresentarem hidrogênio em seu espectro óptico, enquanto que as do Tipo II apresentam estas linhas. Existem também 
subdivisões de acordo com a presença de linhas de outros elementos e a forma da curva de luz. As supernovas do Tipo I são divididas em três subclasses, tipo Ia, Ib e Ic, enquanto que SNs tipo II são divididas em II-P e II-L, segundo suas curvas de luz.

As SNs do tipo Ia acredita-se ocorrerem em sistemas binários onde uma das estrelas evolui tornando-se uma anã branca. Esta estrela pode vir a acretar material da estrela companheira aumentando sua massa até alcançar o limite de Chandrasekhar, $1.44 \mathrm{M}_{\odot}$, a partir do qual não suporta a pressão de degenerescência do elétron e começa o colapso. Isso leva a um processo de nucleossíntese explosiva que destrói a estrela liberando uma grande quantidade de energia. O material é expelido a velocidades da ordem de $5-20 \times 10^{3}$ $\mathrm{km} / \mathrm{s}$. Possuem uma curva de luz característica após a explosão e magnitudes absolutas de $\sim-20$ em seu máximo de brilho (Hillebrandt \& Niemeyer, 2000). Como o pico de luminosidade de suas curvas de luz é o mesmo para todas SNs tipo Ia estas são usadas como indicadores de distâncias de suas galáxias. Seu espectro apresenta como característica a linha de silício ionizado (SiII $\lambda 615.0 \mathrm{~nm}$ ).

As SNs do tipo Ib e Ic não são ainda bem compreendidas. Acredita-se que estes eventos sejam, como as de tipo II, estrelas massivas mas que perdem a maior parte do envelope externo de hidrogênio devido a ventos estelares muito fortes ou interação com uma companheira (Pols \& Nomoto, 1995). Acredita-se que supernovas tipo Ib são o resultado do colapso de uma estrela massiva Wolf-Rayet. Existe alguma evidência de que algumas SNs tipo Ic sejam fontes de raios gama. Estes dois tipos não mostram característica forte de absorção de SiII. As do tipo Ib apresentam linhas de hélio não ionizado enquanto que as do tipo Ic não exibem estas linhas de He. Sua luminosidade é menor do que as do tipo Ia por 1,5-2 magnitudes no máximo de brilho (Weiler \& Sramek, 1988). A taxa de SNs tipo Ib e Ic é muito menor que a taxa de tipo II devido ao fato de serem formadas a partir de estrelas massivas mais raras. Supernovas tipo Ib, Ic e tipo II são chamadas de supernovas de colapso de núcleo, visto que o mecanismo que leva à explosão parece ser similar nestas.

As SNs tipo II, por definição, apresentam hidrogênio em seu espectro óptico e mostramse uma classe diferente de objetos em termos de espectros, de evolução, magnitudes e forma da curva de luz. São estrelas massivas $\left(M>8 M_{\odot}\right)$ que tornam-se instáveis no fim 
de sua vida, colapsando e ejetando seus envelopes externos em uma explosão. Quando a sequência do processo de queima nuclear produz um núcleo de ferro, como não há liberação de energia com a fusão termonuclear deste, o núcleo central contrai. Quando a massa deste núcleo atinge o limite de Chandrasekhar, $1.44 M_{\odot}$, os elétrons tornam-se ultra relativísticos e não mais suportam o núcleo auto-gravitante. A contração produz uma fotodissociação do ferro $\left(\gamma+{ }^{56} \mathrm{Fe} \rightarrow 13{ }^{4} \mathrm{He}+4 n\right)$, processo que consome energia. Esta perda de energia reduz a contribuição térmica da pressão de elétrons, iniciando o colapso.

A partir do processo de captura de nêutrons por núcleos pesados, neutrinos são gerados e escapam da estrela carregando energia. Durante o colapso o núcleo se divide em um núcleo interno aproximadamente homogêneo $\left(M \approx 0.6 \mathrm{M}_{\odot}\right)$ e um externo que colapsa supersonicamente com um perfil de velocidade, $v \propto r^{-1 / 2}$. Em questão de uma fração de segundo o caroço interno colapsante atinge densidades núcleos atômicos. Na borda externa deste gera-se uma onda de choque devido à queda de material do núcleo externo a velocidades supersônicas. Quanto mais matéria cai no caroço interno, mais energia é adicionada ao choque que então começa a se propagar para fora através do núcleo externo. Este choque ejetará o manto estelar para fora do que era o núcleo de ferro, esta reação ao colapso gera a explosão de supernova (Raffelt, 1996).

Baseados em suas curvas de luz podemos separá-las em duas subclasses, tipo II-P que apresentam um platô em sua curva de luz e tipo II-L, que apresentam decaimento linear. A diferença na forma da curva de luz acredita-se ser causada, no caso do tipo II-L, pela expulsão da maior parte do envelope de hidrogênio da estrela progenitora. No tipo II-P, o platô é gerado pela mudança de opacidade nas camadas exteriores. O choque que atravessa o gás ioniza o hidrogênio que é opaco aos fótons que saem do interior. Quando o hidrogênio esfria e recombina as camadas tornam-se transparentes. O máximo de brilho destas SNs é mais fraco que das SNs tipo Ia. SNs tipo II-P apresentam maior dispersão em seu máximo enquanto que as de tipo II-L tem um pico uniforme de 2.5 magnitudes mais fraca que as de tipo Ia. Apesar destas diferenças todas SNs liberam aproximadamente $10^{51}$ erg de energia no meio interestelar, além de matéria enriquecida de metais (Doggett \& Branch, 1985). 
Os remanescentes destas explosões consistem de um objeto compacto, uma estrela de nêutrons ou buraco negro, circundado por uma onda de choque aproximadamente esférica que expande rapidamente. Este choque varre o material interestelar ao redor durante uma fase de expansão livre de aproximadamente 2 séculos. A onda então vai para um período de expansão adiabática até que as perdas radiativas tornem-se importantes, inicia-se a fase radiativa. A partir de então a frente de choque resfria lentamente até se misturar com o meio depois de um período de $\sim 10^{6}$ anos.

A energia cinética liberada por um RSN em expansão pode por um lado injetar turbulência em nuvens próximas e levar à sua fragmentação. Mas por outro, induzir a formação estelar devido à compressão de nuvens próximas.

\section{A.2 Taxas de explosões de SNs}

O evento de explosão de uma estrela pode parecer raro e portanto difícil pensar que este seria realmente considerado uma fonte importante de injeção de energia no meio interestelar e conseqüente formação estelar como foi afirmado. A taxa de explosões de SNs de uma região está relacionada à população estelar local e à quantidade de matéria ali presente. Este fenômeno irá ocorrer para todas as estrelas com massa superior a $8 M_{\odot}$ (no caso de SNs tipo II) e para sistemas binários contendo uma estrela anã branca próxima de sua companheira (SNs tipo Ia). Podemos então estimar a taxa de formação de SNs.

O cálculo da taxa de SNs tipo Ia para um caso mais realista calculado por Matteucci \& Recchi ((2001); (veja também Matteucci et al., 2001)) pode ser escrito como

$$
R_{I a}(t)=C_{1} \int_{m_{B, \text { inf }}}^{m_{B, \text { sup }}} \phi\left(m_{B}\right) \int_{\mu_{\min }}^{\mu_{\max }} f(\mu) \psi\left(t-\tau_{m_{2}}\right) d \mu d m_{B}
$$

onde $m_{B}=m_{1}+m_{2}$ é a massa total do sistema binário, $m_{B, \text { inf }}$ e $m_{B, \text { sup }}$ são as massas mínima e máxima para a estrela companheira contribuinte do sistema binário num tempo $t$ e $C_{1}$ é uma constante de normalização. A função $\phi\left(m_{B}\right)$ é a função de massa inicial (initial mass function - IMF) que corresponde ao número de estrelas formadas numa região num dado intervalo de massa $d m, \mu=m_{2} / m_{B}$ é a fração de massa da estrela 
secundária e $f(\mu)$ é a função de distribuição desta razão. A função $\psi\left(t-\tau_{m_{2}}\right)$ é a taxa de formação estelar (star formation rate - SFR) calculada num intervalo de tempo $\left(t-\tau_{m_{2}}\right)$, com $\tau_{m_{2}}$ sendo o tempo para a explosão. Vemos assim que a época correspondente ao máximo de explosões não é correspondente ao tempo de formação das anãs brancas, mas sim ao tempo de vida das estrelas companheiras $\left(\tau_{\mathbf{m}_{\mathbf{2}}}\right)$. Segundo esta teoria, dependendo do modelo de SFR escolhido pode-se ter uma taxa de SNs Ia de uma em cada 5 bilhões de anos ou até menos (Matteucci et al., 2001; Matteucciet al., 2006).

No caso de SNs tipo II precisamos conhecer o número de estrelas que possuem massa maior que $8 M_{\odot}$. Temos então

$$
N_{S N I I}=\int_{8 M_{\odot}}^{100 M_{\odot}} C_{2} \phi(m) d m
$$

onde o limite de integração inferior refere-se à massa mínima que uma estrela precisa para se tornar uma supernova tipo II, e o limite superior é incerto e vem de estimativas feitas a partir de estrelas quentes e luminosas. Acima de $100 M_{\odot}$ a pressão de radiação domina a gravidade e as estrelas tornam-se instáveis (Maciel, 2005). A função $\phi(m)$ é a IMF e $C_{2}$ é uma constante de normalização. Temos então a taxa de SNs II ao se dividir o número total de estrelas que podem se tornar uma SN pelo tempo máximo necessário para que uma estrela de $8 M_{\odot}$ se forme, em média, $t=30$ Milhões de anos. Assim

$$
R_{S N I I}=\frac{N_{S N I I}}{t} .
$$

Porém, a frequência de ocorrências de ambos os tipos de supernovas na Galáxia pode também ser estimada a partir do monitoramento observacional de sua taxa de ocorrências em um grande número de outras galáxias similares à nossa. Várias observações independentes de SNs podem ser combinadas para se derivar uma frequência média de ex- plosões (Cappellaro et al., 1997, 1999; Ferrière, 2001), onde $(0.38 \pm 0.14) h^{2} S N u$ ou $0.21 \pm 0.08 S N u$ para supernovas tipo I e $(1.53 \pm 0.62) h^{2} S N u$ ou $0.86 \pm 0.35 S N u$ para supernovas tipo II em galáxias Sbc e Sb e onde $h$ é a constante de Hubble em unidades de $100 \mathrm{~km} \mathrm{~s}^{-1} \mathrm{Mpc}^{-1}$ e SNu é a unidade que representa $1 \mathrm{SN} \times\left(10^{10} L_{B} / L_{\odot}\right)^{-1} \times(100 \text { anos })^{-1}$. Escolhendo um valor médio para $h=0.75$ e assumindo que uma galáxia Sbc tem uma luminosidade 
média no azul de $2 \times 10^{10}\left(L_{B}\right) \odot$ encontramos para a taxa de SNs tipo I

$$
\sigma_{S N I} \sim \frac{1}{250 \text { anos }}
$$

e para supernovas tipo II

$$
\sigma_{S N I I} \sim \frac{1}{60 \text { anos }}
$$

Vemos assim que a taxa de SN tipo II é maior que a de SN tipo I e portanto, aquelas terão maior influência na geração de turbulência no meio interestelar. Iremos assim neste trabalho (Cap. 2) explorar a interação destas SNs tipo II com nuvens e verificar sua influência na formação estelar.

\section{A.3 Formação do Remanescente de SN}

Em uma explosão de supernova (SN) uma estrela ejeta uma massa no meio interestelar (MIS) $M_{e j} \approx 1-10 \mathrm{M}_{\odot}$ com velocidade terminal $v_{e j} \approx 10^{4} \mathrm{~km} / \mathrm{s}$ e energia cinética $E_{0} \approx$ $10^{51} \mathrm{erg}$. Esta massa ejetada expande a velocidade aproximadamente constante (expansão livre) até encontrar uma massa equivalente de gás interestelar ambiente produzindo um remanescente de supernova (RSN). Isto ocorre num tempo (McCray, 1985)

$$
t_{0} \approx 200\left(\frac{M_{e j}}{M_{\odot}}\right)^{1 / 3} \frac{1}{n^{1 / 3}} \text { anos }
$$

onde $n$ é a densidade numérica ambiente $\mathrm{em}^{-3}$, e num raio

$$
R_{0} \approx 2\left(\frac{M_{e j}}{M_{\odot}}\right) \frac{1}{n^{1 / 3}} \mathrm{pc} .
$$

Uma onda de choque gerada na superfície de impacto se propaga no gás interestelar. Em algumas vezes o tempo $t_{0}$ a massa ejetada é freada e tem a maior parte de sua energia cinética transferida para este choque que continua a expandir varrendo o gás interestelar por milhares de anos. Sua evolução é caracterizada por duas fases, a adiabática (ou de Sedov-Taylor) e a radiativa. Considerando primeiro a fase inicial de expansão do RSN usamos como primeira aproximação que o gás interestelar ambiente nas vizinhanças do 
remanescente possui densidade uniforme, $\rho=\mu m_{H} n$ e temperatura constante, $T$. Estas simplificações estão em bom acordo com modelos teóricos mais detalhados apesar de a suposição de uma densidade do gás ambiente uniforme limitar a aplicabilidade deste modelo McCray (1985).

Assumimos que a massa do sistema, dominada pela massa do gás interestelar varrido, encontra-se dentro de uma casca esférica fina de raio $R_{R S N}(t)$ expandindo-se a uma velocidade hipersônica de modo que (McCray, 1985)

$$
M_{R S N}(t)=\frac{4 \pi}{3} \rho R_{R S N}(t)^{3}
$$

De acordo com as condições de Rankine-Hugoniot (de Gouveia Dal Pino, 1995) para um gás monoatômico adiabático, o gás chocado na casca esférica terá uma densidade

$$
\rho_{1}=4 \rho
$$

e temperatura

$$
T_{1}=\frac{3}{16} \frac{\mu m_{H}}{k_{B}} v_{R S N}^{2}
$$

onde

$$
v_{R S N}=\frac{d R_{R S N}(t)}{d t}=\dot{R}
$$

Podemos também estimar a espessura da casca a partir da condição

$$
4 \pi R^{2} \Delta R \rho_{1}=\frac{4 \pi}{3} R^{3} \rho
$$

como sendo

$$
\Delta R(t) \approx \frac{R_{R S N}(t)}{12}
$$

Determinamos então o raio do $\mathrm{RSN}, R_{R S N}(t)$, e sua velocidade de expansão, $v_{R S N}$, a partir da segunda Lei de Newton 


$$
F=\frac{d}{d t}(\Delta M \dot{R})
$$

onde

$$
\Delta M=\frac{4 \pi}{3} R^{3} \rho
$$

Então

$$
F=\frac{d}{d t}\left(\frac{4 \pi}{3} R^{3} \rho \dot{R}\right)
$$

A força que empurra o remanescente devido à pressão interna é dada por

$$
F=P 4 \pi R^{2}
$$

onde $P$ é a pressão do gás no interior da casca esférica. Igualando (A.15) e (A.16), temos

$$
\frac{d}{d t}\left(\frac{1}{3} R^{3} \rho \dot{R}\right)=P R^{2}
$$

Ao assumirmos que no início da expansão a energia é principalmente térmica e que a pressão interior é uniforme teremos,

$$
P=\frac{2}{3} \frac{E_{0}}{\frac{4}{3} \pi R^{3}}=\frac{E_{0}}{2 \pi R^{3}},
$$

encontramos, então, que o raio do remanescente em expansão adiabática em função do tempo é a solução da equação diferencial (A.17) dado por

$$
R_{R S N, a}(t)=\left(\frac{25 E_{0} t^{2}}{4 \pi \rho}\right)^{1 / 5} \sim 13\left(\frac{E_{51}}{n}\right)^{1 / 5} t_{4}^{2 / 5} p c
$$

onde $t_{4}$ é o tempo em unidades de $10^{4}$ anos, $E_{51}$ é energia da SN em unidades de $10^{51}$ erg e $\rho=\mu m_{H} n$ é a densidade do gás ambiente e $m_{H}=1.67 \times 10^{-24}$ g é a massa do hidrogênio. Desta equação podemos derivar a velocidade de expansão do gás chocado

$$
v_{R S N, a}(t)=\dot{R} \sim 507\left(\frac{E_{51}}{n}\right)^{1 / 5} t_{4}^{-3 / 5} \mathrm{~km} / \mathrm{s} .
$$


Uma expressão para a temperatura do gás chocado pode ser encontrada substituindo a equação (A.20) acima em (A.10):

$$
T_{R S N}(t)=3.3 \times 10^{6}\left(\frac{E_{51}}{n}\right)^{2 / 5} t_{4}^{-6 / 5} \mathrm{~K} .
$$

Usando (A.19) e (A.20) temos

$$
v_{R S N, a}(R) \sim 68\left(\frac{E_{51}}{n}\right)^{1 / 2} \frac{1}{R_{R S N, 50}^{3 / 2}} \mathrm{~km} / \mathrm{s}
$$

onde $R_{R S N, 50}$ é o raio do RSN em unidades de 50 pc.

Até este ponto consideramos as perdas radiativas como desprezíveis, a casca esférica permanece bastante quente $\left(T>10^{6} \mathrm{~K}\right)$ emitindo raios-X moles por aproximadamente $10^{4}$ anos, mas do cálculo da potência instantânea irradiada podemos encontrar que esta cresce com o tempo de forma que, em um tempo $t_{\text {cool }}$, a aproximação usada acima deixa de ser válida. Isto ocorre quando (McCray, 1985)

$$
\int L(t) d t \approx 0.3 E_{0}
$$

ou seja, quando a fase de conservação de energia termina.

Sabendo que a potência irradiada pela casca é da forma

$$
L(t)=4 \pi R^{2}(t) \Delta R n_{1}^{2} \Lambda(T),
$$

onde $\Delta R$ é dada por (A.12) e $n_{1}=4 n$ vem da equação (A.9) que substituindo em (A.24) nos dá

$$
L(t)=\frac{16 \pi}{3} R^{3} n^{2} \Lambda(T)
$$

onde $\Lambda(T)$ é a função resfriamento para $T>10^{5} \mathrm{~K}$ dada por (McCray, 1985)

$$
\Lambda(T)=10^{-22} T_{6}^{-0.7}+2.3 \times 10^{-24} T_{6}^{0.5} \mathrm{erg} \mathrm{cm}^{3} \mathrm{~s}^{-1} .
$$

Encontramos então um tempo $t_{\text {cool }}$, a partir do qual as perdas radiativas tornam-se importantes. 


$$
t_{\text {cool }}=3 \times 10^{4} E_{51}^{0.22} n^{-0.55} \text { anos }
$$

Após este tempo a casca do remanescente torna-se fria, densa e muito fina, continuando a expansão empurrada pelo gás pouco denso e muito quente do interior do RSN. A pressão no interior diminui segundo a lei adiabática para um gás monoatômico ideal com razão entre os calores específicos $\gamma=5 / 3$,

$$
P V^{5 / 3}=\text { const. }
$$

ou seja,

$$
P=P_{1}\left(\frac{R_{\text {cool }}}{R}\right)^{5}
$$

onde

$$
P_{1}=\frac{2}{3} \frac{E_{0}}{\frac{4}{3} \pi R^{3}}=\frac{E_{0}}{2 \pi R_{\text {cool }}^{3}}
$$

e onde $R_{\text {cool }}$ é o raio do RSN em $t_{c o o l}$ :

$$
R_{\text {cool }}=20 E_{51}^{0.29} n^{-0.42} \mathrm{pc}
$$

Substituindo a expressão (A.29) em (A.17) podemos descrever a evolução do RSN após entrar na fase radiativa por

$$
R_{R S N, r}(t) \simeq\left(\frac{147 E_{0} R_{\text {cool }} t^{2}}{4 \pi \rho}\right)^{1 / 7} \sim 19\left(\frac{E_{51}^{0.23}}{n^{0.26}}\right) t_{4}^{2 / 7} \mathrm{pc} .
$$

A velocidade de expansão nesta fase é dada por

$$
v_{R S N, r}(t) \sim 530 \frac{E_{51}^{0.23}}{n^{0.26}} t_{4}^{-5 / 7} \mathrm{~km} / \mathrm{s} .
$$

Usando (A.31) e (A.32) para eliminar $t_{4}$ temos:

$$
v_{R S N, r}(R) \sim 47 \frac{E_{51}^{0.8}}{n^{0.91} R_{R S N, 50}^{5 / 2}} \mathrm{~km} / \mathrm{s} .
$$


O remanescente continua a expandir por $t>1$ milhão de anos atingindo raios maiores que 60 pc (McCray, 1985). Quando a pressão da casca torna-se comparável à pressão do meio, o gás fragmenta-se e começa a misturar-se com o gás do meio ambiente. Isto ocorre quando

$$
P_{1}\left(\frac{R_{\text {cool }}}{R}\right)^{5}=\rho \frac{k_{B} T}{\mu m_{H}},
$$

onde $T$ e $\rho$ são a temperatura e densidade do meio ambiente. Temos assim que o raio do remanescente nesta fase é dado por

$$
R_{R S N} \sim 69 \frac{E_{51}^{0.32}}{T_{4}^{0.2} n^{0.37}} \mathrm{pc}
$$

onde $T_{4}$ é a temperatura do meio interestelar em unidades de $10^{4} \mathrm{~K}$.

Vimos então que a expansão de um remanescente de supernova possui duas fases distintas, uma fase adiabática (denominada de Sedov-Taylor) e uma fase radiativa. Na primeira, como não há perdas consideráveis de energia a frente de choque possui maior força e veremos (Caps. 2 e 3) que será capaz de, ao encontrar uma nuvem, fragmentá-la, levando à formação de glóbulos densos, ou até mesmo de destruí-la, fazendo com que seu material se misture ao meio com mais facilidade que um RSN na fase radiativa. 


\section{Apêndice B}

\section{Código Numérico 3D MHD-Godunov}

Para a realização das simulações numérica 3D MHD apresentadas nesta tese, utilizamos uma versão modificada do código numérico 3D MHD-Godunov originalmente escrito por Grzegorz Kowal o qual foi testado e utilizado em diversos estudos de turbulência MHD no MIS e no meio intergaláctico (e.g. Kulesza-Żydzik et al., 2009; Kulpa-Dybeł et al., 2011) ; em aglomerados de galaxias (e.g., Falceta-Gonçalves et al. 2010a, 2010b); de formação estelar (e.g., Leão et al. 2009; 2012; Santos-Lima et al. 2010, 2012; de Gouveia Dal Pino et al. 2012) de reconexão magnética (e.g., Kowal et al. 2009; 2012); de aceleração de raios cósmicos (e.g., Kowal et al. 2011; Kowal et al. 2012); etc. A seguir, apresentaremos uma breve descrição do mesmo (para maiores detalhes veja Kowal et al., 2009, http://daphne.iag.usp.br/gitweb).

\section{Equações MHD}

O código usa um esquema tipo Godunov baseado em reconstrução espacial não oscilatória $(E N O)^{1}$ e integração temporal de Runge-Kutta (RK) de segunda ordem (veja Londrillo \& Del Zanna, 2000; Del Zanna et al, 2003) para resolver as equações isotérmicas MHD

\footnotetext{
${ }^{1}$ ENO, do inglês, 'essentially non oscilatory', são esquemas de diferenças finitas de alta ordem projetados para problemas com soluções seccionalmente suaves contendo descontinuidades (Shu et al. 1992)
} 
em sua forma ideal,

$$
\begin{gathered}
\frac{\partial \rho}{\partial t}+\nabla \cdot(\rho \mathbf{v})=0 \\
\rho\left(\frac{\partial}{\partial t}+\mathbf{v} \cdot \nabla\right) \mathbf{v}=-c_{s}^{2} \nabla \rho+\frac{1}{4 \pi}(\nabla \times \mathbf{B}) \times \mathbf{B} \\
-\rho \nabla\left(\Psi_{\text {gas }}+\Psi_{\star}\right)+\mathbf{f} \\
\frac{\partial \mathbf{A}}{\partial t}+\mathbf{E}=\mathbf{0},
\end{gathered}
$$

Sendo que a primeira equação descreve a conservação de massa, a segunda a conservação de momentum e a terceira a equação de indução magnética, onde $\rho$ e v são a densidade e a velocidade do plasma, respectivamente, $\mathbf{A}$ é o vetor potencial, $\mathbf{E}=-\mathbf{v} \times \mathbf{B}+\eta \mathbf{j}$ é o campo elétrico, $\mathbf{B} \equiv \nabla \times \mathbf{A}$ é o campo magnético, $\mathbf{j}=\nabla \times \mathbf{B}$ é a densidade de corrente, $c_{s}$ é a velocidade do som isotérmica, $\eta$ é o coeficiente de resistividade, e f representa o termo de 'forcing', i.e., injeção de turbulência. O campo magnético B, satisfaz a condição de divergência $\nabla \cdot \mathbf{B}=0$. Esta é mantida incorporando-se a aproximação de transporte vinculado (CT) do campo magnético interpolado (see Londrillo \& Del Zanna, 2000) na integração da equação de indução magnética (Eq. B.3). O potencial $\Psi_{\text {gas }}$ é devido à auto-gravidade do gás e obedece à equação de Poisson:

$$
\nabla^{2} \Psi_{g a s}=4 \pi G \rho
$$

e $\Psi_{\star}(r)$ é o potencial gravitacional devido a estrelas e para uma simetria esférica é, dado por:

$$
\Psi_{\star}\left(r \leq r_{\text {max }}\right)=-\frac{G M_{\star}}{\left(r+r_{\star}\right)}
$$

onde $r$ é a distância ao centro do domínio computacional, e $r_{\text {max }}$ é um raio de corte (cutoff) na força gravitacional para assegurar a simetria, pois usamos fronteiras periódicas, e $r_{\star}$ é um raio de suavização. 


\section{Resolvedores de Riemann e o método Godunov}

O código emprega os resolvedores de Riemann² ${ }^{2}$ Harten-Lax-van Leer (HLL, Harten et al., 1983) e HLLD (Mignone, 2007) para integrar espacialmente as equações MHD nas células da grade computacional (e.g. Londrillo \& Del Zanna, 2000). Estes esquemas possuem propriedades de dissipação bastante diferentes. O resolvedor HLL foi inicialmente desenvolvido para resolver as equações de Euler e posteriormente adotado para resolver equações diferenciais dependentes do tempo calculando a média do 'leque' de Riemann ${ }^{3}$ sobre uma região entre os mínimos e máximos locais das velocidades características do sistema. Desta forma um estado intermediário foi construído. O resolvedor de Riemann HLLD também considera as descontinuidades resultantes da presença de campos magnéticos, separando este estado intermediário em múltiplos estados intermediários, resolvendo, e.g., ondas de Alfvén com muito menos dissipação.

O esquema Godunov é um método para se resolver o problema de Riemann, o qual é representado pela lei de conservação escalar

$$
\frac{\partial u}{\partial t}+\frac{\partial f}{\partial x}(u)=0
$$

onde $f=f(u)$ é a função fluxo. O esquema Godunov apresenta-se na forma,

$$
u_{i}^{n+1}=u_{i}^{n+1}+\frac{\Delta t}{\Delta x}\left[f_{i-\frac{1}{2}}-f_{i+\frac{1}{2}}\right]
$$

onde

$$
f_{i+\frac{1}{2}}=f_{i+\frac{1}{2}}\left(u_{i-l_{L}}^{n}, \ldots, u_{i+l_{R}}^{n}\right)
$$

\footnotetext{
${ }^{2} \mathrm{O}$ resolvedor de Riemann fornece uma solução acurada para os problemas de interface de células de grade. Esta baseia-se na solução em ambos os lados da interface, e com isso reconstrói-se o fluxo numérico das variáveis conservadas através da interface (veja Toro, 1999)

${ }^{3} \mathrm{O}$ leque de Riemann ocorre quando uma descontinuidade se propaga a uma distância at em um tempo $t$. A curva característica gerada, $x=a t$, irá separar a região da soluções $u_{L}$ da região das soluções $u_{R}$. A solução do problema de Riemann para um sistema linear pode então, ser representada no plano $x-t$ por retas formando um 'leque'.(Toro, 1999)
} 
com $l_{L}$ e $l_{R}$ inteiros não negativos e onde $f_{i+\frac{1}{2}}$ é uma aproximação para o fluxo físico de (B.6) denominado fluxo numérico da variável conservada e $u_{i}^{n+1}$ é a i-ésima variável calculada no passo de tempo $(n+1)$. Este esquema conservativo requer uma redefinição da discretização do domínio, o qual agora trata de médias nas células definidas sobre volumes finitos. Um exemplo do domínio discretizado no plano x-t é mostrado na figura B.1 (Toro, 1999). Neste o comprimento é subdividido em M volumes finitos, chamados de células computacionais, dadas como

$$
x_{i-\frac{1}{2}}=(i-1) \Delta x \leq x \geq i \Delta x=x_{i+\frac{1}{2}} .
$$

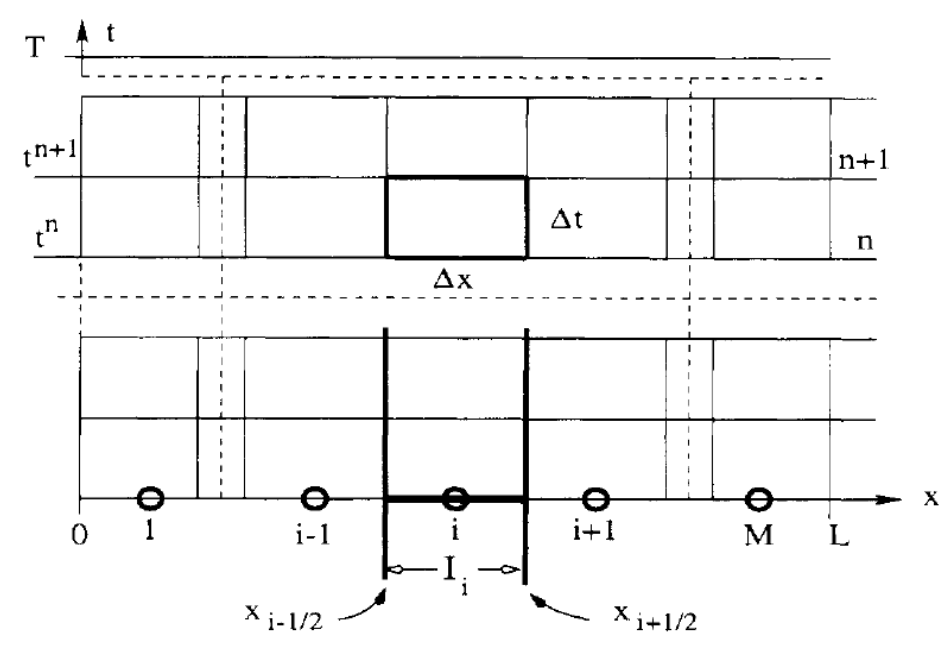

Figura B.1: Discretização do domínio de tamanho L em M volumes finitos $I_{i}$ (células computacionais) (Extraído de Toro (1999)).

$x_{i-\frac{1}{2}}$ e $x_{i+\frac{1}{2}}$ definem as posições das interfaces ou fronteiras das células nas quais os fluxos numéricos precisam ser calculados e o tamanho das células é

$$
\Delta x=x_{i+\frac{1}{2}}-x_{i-\frac{1}{2}}=\frac{L}{M} .
$$

O função de fluxo numérico pode então ser calculada usando soluções locais para o problema de Riemann. Para isso supõe-se que em um dado tempo $n$, têm-se uma distribuição constante definida por partes, como apresentado na figura B.2. Desta forma, pode-se resolver o problema como pares de estados constantes $\left(u_{i}^{n}, u_{i+1}^{n}\right)$ separados por 
uma descontinuidade na fronteira da célula $x_{i+\frac{1}{2}}($ Toro, 1999).

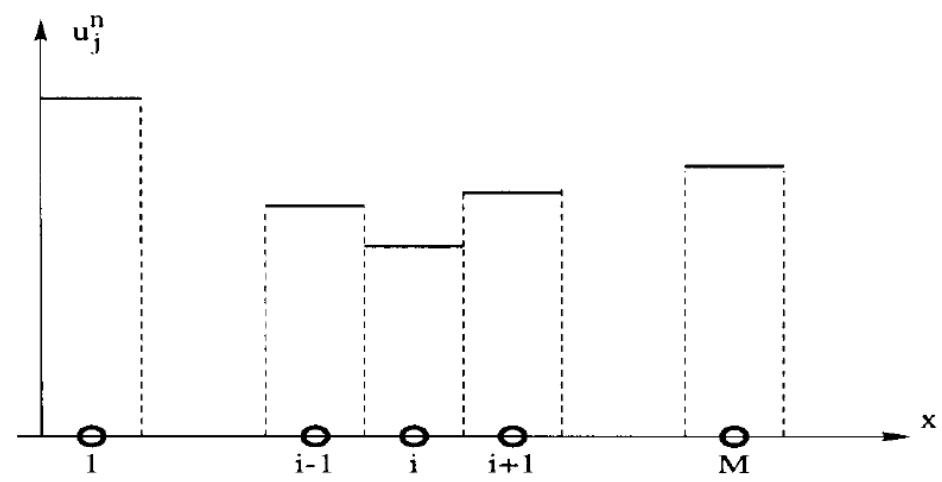

Figura B.2: Distribuição das constantes definidas por partes em um dado passo de tempo (Extraído de Toro (1999)).

No código usam-se as coordenadas espaciais, dadas em unidades de um comprimento típico, $L_{*}$ (que pode ser a dimensão do sistema em estudo, por exemplo, o tamanho da nuvem), a densidade, $\rho$, normalizada por uma densidade de referência $\rho_{*}$ (que pode ser, por exemplo, a densidade do meio ambiente), o campo de velocidades, $\mathbf{v}$, e a velocidade do som, $c_{s}$, por uma velocidade de referência, $v_{*}$ (que pode ser, por exemplo, a própria velocidade do som do meio). Com essas unidades de referência podemos encontrar todas as outras variáveis, em especial, o campo magnético é dado em unidades de $v_{*} \sqrt{4 \pi \rho_{*}}$.

Usamos condições periódicas para as fronteiras do domínio computacional empregado neste trabalho.

\section{Injeção de turbulência}

O 'forcing', f, é a força responsável pela injeção de turbulência no domínio computacional. Este é implementado no espaço espectral de Fourier concentrado ao redor de um vetor de onda $k_{i n j}$ correspondendo à escala de injeção $l_{i n j}$. Perturba-se um número $N_{f}$ de componentes discretas da velocidade em uma casca esférica de espessura de $k_{i n j}-\Delta k_{i n j}$ a $k_{i n j}+\Delta k_{i n j}$ com um perfil Gaussiano de meia altura $k_{c}$ e pico $\tilde{v}_{f}=v_{\text {turb }}$ na escala de injeção. Em todos os modelos aqui estudados adotamos $k_{c}=0.4$ em unidades do código.Como perturbamos um número discreto $N_{f}$ de componentes Fourier que dependem 
da escala de injeção $k_{i n j}$ e da espessura da casaca perturbada $\Delta k_{i n j}$, a amplitude $\tilde{v}_{f}$ varia com a escala de injeção de modo a manter sempre a mesma potência de injeção de turbulência $P_{i n j}$. 


\section{Apêndice $\mathrm{C}$}

Local star formation triggered by supernova shocks in magnetized diffuse neutral clouds 


\section{Apêndice D}

Magnetic flux transport by turbulent reconnection in astrophysical flows 


\section{Apêndice E}

Cloud core collapse and the role of turbulent magnetic reconnection diffusion 


\section{Referências Bibliográficas}

Alves F. O., Girart J. M., Lai S.-P., Rao R., Zhang Q. 2011, ApJ, 726, 63

Aldrovani, S. M. V., \& Péquignot, D. 2000 A\&A, 25, 137

Alvelius, K. 1999, Physics of Fluids, 11, 1880

Anderson, M. C., Jones, T. W., Rudnick, L., Tregillis, I. L., \& Kang, H. 1994, ApJ, 421, L31

Armstrong, J. W., Rickett, B. J., \& Spangler, S. R. 1995, ApJ, 443, 209

de Avillez, M. A. 2000, Ap\&SS, 272, 23

de Avillez, M. A., \& Berry, D. L. 2001, MNRAS, 328, 708

de Avillez, M. A., \& Breitschwerdt, D. 2005, A\&A, 436, 585

Ballesteros-Paredes, J. 2006, MNRAS, 372, 443

Ballesteros-Paredes J., Klessen R. S., Mac Low M.-M., Vazquez-Semadeni E., 2007, prpl.conf, 63

Bedogni R., Woodward P. R., 1990, A\&A, 231, 481

Biskamp, D. 1986, Physics of Fluids, 29, 1520

Blitz, L. 1993, Protostars and Planets III, 125

Bonazzola, S., Heyvaerts, J., Falgarone, E., Perault, M., \& Puget, J. L. 1987, A\&A, 172, 293 
Bonnell, I. A., Dobbs, C. L., Robitaille, T. P., \& Pringle, J. E. 2006, MNRAS, 365, 37

Cappellaro, E., Turatto, M., Tsvetkov, D. Yu., Bartunov, O. S., Pollas, C., Evans, R. \& Hamuy, M. 1997, A\&A, 322, 431

Cappellaro, E., Evans, R., \& Turatto, M. 1999, A\&A, 351, 459

Carswell, R. F., Becker, G. D., Jorgenson, R. A., Murphy, M. T. \& Wolfe, A. M. 2012, MNRAS, 422, 1700

Carroll, B. W., \& Ostlie, D. A. 1996, Institute for Mathematics and Its Applications,

Cerqueira, A.H., de Gouveia Dal Pino, E. M., \& Herant, M. 1997, ApJ, 489, L185

Cerqueira, A.H., \& de Gouveia Dal Pino, E. M. 2001, ApJ, 550, L91

Cerqueira, A.H., \& de Gouveia Dal Pino, E. M. 2001, ApJ. , 560, 799

Cerqueira, A.H., de Gouveia Dal Pino, E. M. 2004, A\&A., 426, L25

Chaisson, E., \& McMillan, S. 2005, Astronomy Today, Volume 2: Stars and Galaxies, 5th Edition, by E. Chaisson and S. McMillan. Prentice Hall, 2005. ISBN 0-13-117684-6.,

Chandrasekhar, S.: Hidrodynamics and Hidromagnetics Stability. Dover Publications, Inc., New York, 1961

Chernin, L., Masson, C., de Gouveia Dal Pino, E. M., \& Benz, W. 1994, ApJ, 426, 261

Cho J., Lazarian A., Yan H., 2002, ASPC, 276, 170

Cox, D. P., \& Smith, B. W. 1974, ApJ, 189, L105

Cox, D. P. 2005, ARA\&A, 43, 337

Crutcher, R. M. 1991, ApJ, 520, 706

Crutcher, R. M. 1999, ApJ, 514, L121

Crutcher, R. M. 2005, Massive Star Birth: A Crossroads of Astrophysics, 227, 98 
Crutcher, R. M. 2005, The Magnetized Plasma in Galaxy Evolution proc., 103

Crutcher, R. M. 2005, Magnetic Fields in the Universe: From Laboratory and Stars to Primordial Structures., 784, 129

Crutcher, R. M. 2008, Ap\&SS, 313, 141

Crutcher R. M., \& Troland T. H., 2007, IAUS, 237, 141

Crutcher R. M., Hakobian N., Troland T. H., 2009, ApJ, 692, 844

Crutcher R. M., Hakobian N., Troland T. H., 2010, MNRAS, 402, L64

Crutcher, R. M., Wandelt, B., Heiles, C., Falgarone, E., \& Troland, T. H. 2010, ApJ, 725, 466

Dalgarno, A., \& McCray, R. A. 1972, ARA\&A, 10, 375

Del Zanna, L., Bucciantini, N., \& Londrillo, P. 2003, A\&A, 400, 397

Doggett, J. B., \& Branch, D. 1985, Astron. J., 90, 2303

Dopita, M. A., \& Sutherland, R. S. 2003, Astrophysics of the diffuse universe, Berlin, New York: Springer, 2003. Astronomy and astrophysics library, ISBN 3540433627

Draine, B. T., \& McKee, C. F. 1993, ARA\&A, 31, 373

Dubner, G. M., Green, A. J., Goss, W. M., Bock, D. C. J. \& Giacani, E 1998, ApJ, 116, 813

Elmegreen, B. G. 1979, ApJ, 232, 729

Elmegreen, B. G. 2000, ApJ, 530, 277

Elmegreen B. G., Lada C. J., 1977, ApJ, 214, 725

Elmegreen, B. G., \& Elmegreen, D. M. 1978, ApJ, 220, 1051

Elmegreen, B. G., \& Scalo, J. 2004, ARA\&A, 42, 211 
Falceta-Gonçalves, D., Lazarian, A., \& Kowal, G. 2008, ApJ, 679, 537

Falceta-Gonçalves, D., de Gouveia Dal Pino, E. M., Gallagher, J. S., \& Lazarian, A. 2010, ApJ, 708, L57

Falceta-Gonçalves, D., Caproni, A., Abraham, Z., Teixeira, D. M., \& de Gouveia Dal Pino, E. M. 2010, ApJ, 713, L74

Falgarone, E., Troland, T. H., Crutcher, R. M., \& Paubert, G. 2008, A\&A, 487, 247

Fatuzzo, M., \& Adams, F. C. 2002, ApJ, 570, 210

Ferrière, K. M. 2001, RvMPhy., 73(4), 1031

Fiedler, R. A., \& Mouschovias, T. C. 1992, ApJ, 391, 199

Fiedler, R. A., \& Mouschovias, T. C. 1993, ApJ, 415, 680

de Gouveia Dal Pino, E. M.; Plasmas em Astrofísica, Apostila do curso, IAG - USP, 1995.

de Gouveia Dal Pino, E. M. 1999, ApJ, 526, 862

de Gouveia Dal Pino, E. M. 2001, ApJ, 551, 347

de Gouveia Dal Pino, E. M., \& Benz, W. 1993, ApJ, 410, 686

de Gouveia Dal Pino, E. M., \& Benz, W. 1994, ApJ, 435, 204

de Gouveia dal Pino, E. M., \& Tanco, G. A. M. 1999, ApJ, 518, 129

de Gouveia Dal Pino, E. M., Melioli, C., D’Ercole, A., Brighenti, F., \& Raga, A. C. 2009, Revista Mexicana de Astronomia y Astrofisica Conference Series, 36, 17

de Gouveia Dal Pino, E. M., Falceta-Gonçalves, D., Gallagher, J. S., et al. 2010, Highlights of Astronomy, 15, 452

de Gouveia Dal Pino, E. M., Santos-Lima, R., Lazarian, A., Leão, M. R. M., FalcetaGonçalves, D., \& Kowal, G. 2011, IAU Symposium, 274, 333 
de Gouveia Dal Pino, E. M., Leão, M. R. M., Santos-Lima, R., et al. 2012, Phys. Scr, 86, 018401

Gonzalez, R. F., de Gouveia Dal Pino, E. M., Raga, A. C., \& Velazquez, P. F. 2004, ApJ, 600,59

Gonzalez, R. F., de Gouveia Dal Pino, E. M., Raga, A. C., \& Velazquez, P. F. 2004, ApJ, 616,976

Harten, A., Lax, P. D., \& van Leer, B. 1983, SIAM Rev. 25, 35

Haugen, N. E., Brandenburg, A., \& Dobler, W. 2004, Phys. Rev. E, 70, 016308

Hayashi, C. 1966, ARA\&A, 4, 171

Haynes, M. P. \& Broeils, A. H., in The Interstellar Medium in Galaxies, ed. Van der Hulst, J. M., Kluwer Academic Publishers, 1997, p75

Heiles, C., \& Crutcher, R. 2005, Cosmic Magnetic Fields, 664, 137

Heiles, C., \& Troland, T. H. 2005, ApJ, 624, 773

Heitsch, F., Mac Low, M.-M., \& Klessen, R. S. 2001, ApJ, 547, 280

Heckman T. M., Sembach K. R., Meurer G. R., Leitherer C., Calzetti D., Martin C. L., 2001, ApJ, 558, 56

Heydari-Malayeri, M., Kahane, C. \& Lucas, R. 1981, Nature, 293, 549

Hillebrandt,W. \& Niemeyer, J. C. 2000, ARA\&A, 38, 191

Iroshnikov, P. S. 1963, AZh, 40, 742

Joung, M. K. R., \& Mac Low, M.-M. 2006, ApJ, 653, 1266

Klein, R. I., McKee, C. F. , \& Colella, P. 1994, ApJ, 420, 213

Klessen, R. S., Heitsch, F., \& Mac Low, M.-M. 2000, ApJ, 535, 887 
Kobayashi, N \& Tokunaga, A. T. 2000, ApJ, 532, 423

Kobayashi, N., Yasui, C., Tokunaga, A. T., \& Saito, M. 2008, ApJ, 683, 178

Kornreich, P., \& Scalo, J. 2000, ApJ, 531, 366

Kowal, G., Lazarian, A., \& Beresnyak, A. 2007, ApJ, 658, 423

Kowal, G., \& Lazarian, A. 2007, ApJ, 666, L69

Kowal, G., Lazarian, A., Vishniac, E. T., \& Otmianowska-Mazur, K. 2009, ApJ, 700, 63

Kowal, G., de Gouveia Dal Pino, E. M., \& Lazarian, A. 2011, ApJ, 735, 102

Kowal, G., de Gouveia Dal Pino, E. M., \& Lazarian, A. 2012, Physical Review Letters, 108,241102

Kraichnan, R. H. 1965, Physics of Fluids, 8, 1385

Krasnopolsky, R., Li, Z.-Y., \& Shang, H. 2010, ApJ, 716, 1541

Krasnopolsky, R., Li, Z.-Y., \& Shang, H. 2011, ApJ, 733, 54

Kritsuk, A. G., \& Norman, M. L. 2002, ApJ, 569, L127

Kritsuk, A. G., \& Norman, M. L. 2002, ApJ, 580, L51

Kulesza-Żydzik, B., Kulpa-Dybeł, K., Otmianowska-Mazur, K., Kowal, G., \& Soida, M. 2009, A\&A, 498, L21

Kulpa-Dybeł, K., Otmianowska-Mazur, K., Kulesza-Żydzik, B., et al. 2011, ApJ, 733, L18

Johns-Krull, C. M. 2007, ApJ, 664, 975

Lada, C. J., \& Lada, E. A. 2003, ARA\&A, 41, 57

Landau L. D., \& Lifshitz E. M. 1987, Fluid Mechanics, Oxford: Pergamon Press

Lazarian, A. 2005, Magnetic Fields in the Universe: From Laboratory and Stars to Primordial Structures., 784, 42 
Lazarian, A. 2006, ApJ, 645, L25

Larazian, A. 2011, arXiv:1108:2280v1

Larazian, A. 2012, Magnetic Fields in the Universe III: From Laboratory and Stars to Primordial Structures., in press

Lazarian, A., Santos-Lima, R., \& de Gouveia Dal Pino, E. M 2010, Numerical Modeling of Space Plasma Flows, Astronum-2009, 429, 113

Lazarian, A., \& Vishniac, E. T. 1999, ApJ, 517, 700

Lazarian, A., Vishniac, E. T., \& Cho, J. 2004, ApJ, 603, 180

Leão, M. R. M. "Formação estelar induzida por choque de Supernovas", Dissertação de Mestrado, IAG/USP, São Paulo, 2007.

Leão, M. R. M., de Gouveia Dal Pino, E. M., Falceta-Gonçalves, D., Melioli, C., \& Geraissate, F. G. 2009, MNRAS, 394, 157

Leão, M. R. M., de Gouveia Dal Pino, E. M., Santos-Lima, R., \& Lazarian, A. 2012, ApJ, submited

Ledoux, P., AnAp, 1951, 14, 438

Levy, D., Puppo, G., \& Russo, G. 1999, Mathematical Modelling and Numerical Analysis, 33,547

Li, P. S., McKee C. F., Klein, R. I.,\& Fisher, R. T. 2008, ApJ684 380

Li, Z.-Y., Krasnopolsky, R., \& Shang, H. 2011, ApJ, 738, 180

Lizano, S., \& Shu, F. H. 1989, ApJ, 342, 834

Liu, X.-D., \& Osher, S. 1998, Journal of Computational Physics, 141, 1

Londrillo, P., \& Del Zanna, L. 2000, ApJ, 530, 50

Maciel,W. J., Astrofísica do Meio Interestelar, São Paulo, Editora da USP, 2002. 
Maciel,W. J., Evolução Química da Galáxia, Apostila do curso, São Paulo, 2005.

Mac Low, M.-M. 2009, Revista Mexicana de Astronomia y Astrofisica Conference Series, 36,121

Mac Low, M.-M., \& Klessen, R. S. 2004, Reviews of Modern Physics, 76, 125

Madsen, G.J., Reynolds, R.J., Haffner, L.M. 2002, AAS 199th meeting, Washington, DC

Masciadri, E., de Gouveia Dal Pino, E. M., Raga, A. C., \& Noriega-Crespo, A. 2002, ApJ, 580,950

Mathis, J. S. 2000, ApJ, 544, 347

Matteucci, F. \& Recchi, S. 2001, ApJ, 558, 351

Matteucci, F., Panagia, N., Pipino, A., Mannucci, F., Recchi, S. \& Della Valle M. 2006, MNRAS, 372, 265

McCray, R., \& Snow, T. P., Jr. 1979, ARA\&A, 17, 213

McCray, R., in Spectroscopy of Astrophysical Plasmas, editado por A. Dalgarno \& D. Layzer, p270, 1985.

McKee, C. F., in Spectroscopy of Astrophysical Plasmas, editado por A. Dalgarno \& D. Layzer, p209, 1985.

McKee, C. F., \& Cowie, L. L. 1975, ApJ, 195, 715

McKee, C. F. \& Otriker, J. P. 1977, ApJ, 218, 148

McKee, C. F., \& Holliman II, J. H. 1999, ApJ, 522, 313

McKee, C. F., \& Ostriker, E. C. 2007, ARA\&A, 45, 565

Melioli, C., Evolução e energização do gás por supernovas em ambientes de intensa formação estelar, Tese de Doutorado, IAG/USP, São Paulo, 2006.

Melioli, C., \& de Gouveia Dal Pino, E. M. 2004, A\&A, 424, 817 
Melioli, C., de Gouveia Dal Pino, E. M. \& Raga, A. 2005, A\&A, 443, 495

Melioli, C., \& de Gouveia Dal Pino, E. M. 2006, A\&A, 445, L23

Melioli, C., de Gouveia Dal Pino, E. M., de la Reza, R. \& Raga, A. C. 2006, MNRAS, 373,811

Melioli, C., Brighenti, F., D'Ercole, A., \& de Gouveia Dal Pino, E. M. 2008, MNRAS, 388,573

Melioli, C., Brighenti, F., D’Ercole, A., \& de Gouveia Dal Pino, E. M. 2009, MNRAS, 399, 1089

Mestel, L., \& Spitzer, L., Jr. 1956, MNRAS, 116, 503

Mignone, A. 2007, Journal of Computational Physics, 225, 1427

Mishra, S. K., Sodha, M. S., \& Srivastava, S. 2013, Ap\&SS, 344, 193

Mouschovias, T. C. 1976, ApJ, 207, 141

Mouschovias, T. C. 1977, ApJ, 211, 147

Mouschovias, T. C. 1979, ApJ, 228, 475

Mouschovias, T. Ch 1995, ASPC, 80, 184

Mouschovias, T. Ch. \& Paleologou, E. V. 1981, ApJ, 246, 48

Myers, P. C. \& Fuller, G. A. 1993, ASPC, 35, 70

Nakamura, F., \& Li, Z.-Y. 2005, ApJ, 631, 411

Nakamura, F., McKee, C. F.; Klein, R.I. \& Fisher, R. T. 2006, ApJS, 164, 477

Nakano, T. 1984, Fund. Cosmic Physics, 9, 139

Nakano, T., \& Nakamura, T. 1978, PASJ, 30, 671

Nakano, T., \& Tademaru, E. 1972, ApJ, 173, 87 
Norman, C. A., \& Ferrara, A. 1996, ApJ, 467, 280

Oliveira Filho, K. S. \& Oliveira Saraiva, M. F., Astronomia \&3 Astrofísica, 2. ed., São Paulo, Editora Livraria da Física.

Ortega, V. G., de la Reza, R., Jilinski, E. \& Bazzanella, B. 2004, ApJ, 576, 832

Parker, E. N. 1958, ApJ, 128, 664

Particle Physics Booklet, resumo do Review of Particle Physics, S. Eidelman et al. 2004, Phy.L, B 592, 1

Petschek, H. E. 1964, NASA Special Publication, 50, 425

Polifke, W., \& Shtilman, L. 1989, Physics of Fluids, 1, 2025

Pols, O. \& Nomoto, K. 1995, Proceedings of the The Third Pacific Rim Conference on Recent Development on Binary Star Research, 153

Poludnenko, A. Y., Frank, A. \& Blackman, E. G. 2002, ApJ, 576, 832

Press, W. H, Teukolsky, S. A., \& Flannery, B. P. 1992, Numerical Recipies in Fortran 77: The Art of Scientific Computing., Vol.1, by William H. Press - 2nd. ed., Published by Press Syndicate of University of Cambridge, ISBN 0-521-43064-X.

Raffelt, G. G. 1996, Star as Laboratories for Fundamental Physics: the astrophysics of neutrinos, axions, and other weakly particles, Univ. of Chicago Press

Raga, A. C., Navarro-Gonzalez, R., \& Villagran-Muniz, M. 2000, RMxAA., 36, 67

Raga, A. C., de Gouveia Dal Pino, E. M., Noriega-Crespo, A. \& Velazquez, P. F. 2002, A\&A, 392, 267

Rand, R. J., 1997, in The Interstellar Medium in Galaxies, ed. Van der Hulst, J. M., Kluwer Academic Publishers, p105

Reynolds, R. J., Haffner, L. M. \& Tufte, S. L. 2000., Revista Mexicana de Astronomia y Astrofisica Conference Series, ed. Arthur, S. J., Brickhouse, N. S. \& Franco, J., 9, 249 
Reynoso, E. M. \& Mangum, J. G. 2001, ApJ, 121, 347

Roberts, W. W. 1969, ApJ, 158, 123

Ruffle, P. M. E., Millar, T. J., Roberts, H., Lubowich, D. A., Henkel, C., Pasachoff, J. M. \& Brammer, G. 2007, ApJ, 671, 1766

Santos-Lima, R., Lazarian, A., de Gouveia Dal Pino, E. M., \& Cho, J. 2010, ApJ, 714, 442

Santos-Lima, R., de Gouveia Dal Pino, E. M., \& Lazarian, A. 2012, ApJ, 747, 21

Sasaki, M., Kothes, R., Plucinsky, P. P., Gaetz, T. J. \& Brunt, C. M. 2006, ApJ, 642, L149

Sgro A. G., 1975, ApJ, 197, 621

Schulz, N. S. 2005, From Dust to Stars, Praxis Publishing Ltd, Chichester, UK

Shay, M. A., Drake, J. F., Denton, R. E., \& Biskamp, D. 1998, J. Geophys. Res., 103, 9165

Shay, M. A., Drake, J. F., Swisdak, M., \& Rogers, B. N. 2004, Phys. of Plasmas, 11, 2199

Shu, F. H. 1983, ApJ, 273, 202

Shu, F. H. 1985, IAUS, 106, 561

Shu, F. H., The Physics of Astrophysics - Gas Dynamics, Vol. II, Ed University Sci. Books, Mill Valley, California, 1992.

Shu, F. H., Lizano, S., Ruden, S. P., \& Najita, J. 1988, ApJ, 328, L19

Shu, F. H. , Adams, F. C. \& Lizano, S. 1987, ARA\&A, 25, 23

Shu, F. H., Galli, D., Lizano, S., \& Cai, M. 2006, ApJ, 647, 382

Shu, C.-W., Zang, T. A., Erlebacher, G., Whitaker, D., \& Osher, S. 1992, App. Num. Math., 9, 45 
Simon, R. 1965, AnAp, 28, 40

Simon, R. 1965, AnAp, 28, 625

Spaans, M. \& Silk, J., 2000, ApJ, 538, 115

Spitzer, L., Jr. 1968, Nebulae and Interstellar Matter, 1

Stahter, S. W. 1983, ApJ, 268, 155

Steffen W., López J. A., 2004, ApJ, 612, 319

Stone, J. M., Gardner, T. A., Teuben, P., Hawley, J. F. \& Simon, J. B., 2008, ApJ, 178, 137

Stone, J. M., \& Zweibel, E. G. 2010, ApJ, 724, 131

Strittmatter, P. A. 1966, MNRAS, 132, 359

Sweet, P. A. 1958, Electromagn. Phenom. Cosm. Phys., 6, 123

Tassis, K., \& Mouschovias, T. C. 2005, ApJ, 618, 769

Tenorio-Tagle G., Rozyczka M., 1986, å, 155, 120

Toro, Eleuterio F., Riemann solvers and numerical methods for fluid dynamics. A practical introduction. Second edition. Springer-Verlag, Berlin, 1999. 624 pp.

Umebayashi, T. \& Nakano, T. 1980, PASJ, 32, 405

Uzdensky, D. A., \& Kulsrud, R. M. 2000, Phys. of Plasmas, 7, 4018

Vazquez-Semadeni, E., Ostriker, E. C., Passot, T., Gammie, C. F., \& Stone, J. M. 2000, Protostars and Planets IV, 3

Vázquez-Semadeni, E., Kim, J., \& Ballesteros-Paredes, J. 2005, ApJ, 630, L49

Vázquez-Semadeni, E. 2010, Astronomical Society of the Pacific Conference Series, 438, 83 
Vishniac, E. T., \& Lazarian, A. 1999, ApJ, 511, 193

Wada, K., \& Norman, C. A. 2001, ApJ, 547, 172

Wang, Z., Qu, Q., Luo, D., McCray, R. \& Mac Low, M-M. 1992, ApJ, 388, 127

Weiler, K. W. \& Sramek, R. A. 1988, ARA\&A, 26, 295

Williams, J. P., Blitz, L., \& McKee, C. F. 2000, Protostars and Planets IV, 97

Wolfire, M. G., McKee, C. F, Hollenbach, D., Tielens, A. G. G. M. 2003, ApJ, 587, 278

Wolfe A. M., Prochaska J. X., Gawiser E., 2003a, ApJ, 593, 215

Yamada, M., Ren, Y., Ji, H., Breslau, J., Gerhardt, S., Kulsrud, R., \& Kuritsyn, A. 2006, Phys. Plasmas, 13, 052119

Yang, H., \& Johns-Krull, C. M. 2011, ApJ, 729, 83

Yasui, C., Kobayashi, N., Tokunaga, A.T., Terada, H. \& Saito, M. 2006, ApJ, 649, 753

Zweibel, E. G. 2002, ApJ, 567, 962 